University of Louisville

ThinkIR: The University of Louisville's Institutional Repository

Electronic Theses and Dissertations

$12-2003$

\title{
Studies of the effects of pancreatic beta cell antioxidant transgenes on experimental models of diabetes.
}

Hainan Chen

University of Louisville

Follow this and additional works at: https://ir.library.louisville.edu/etd

\section{Recommended Citation}

Chen, Hainan, "Studies of the effects of pancreatic beta cell antioxidant transgenes on experimental models of diabetes." (2003). Electronic Theses and Dissertations. Paper 235.

https://doi.org/10.18297/etd/235

This Doctoral Dissertation is brought to you for free and open access by ThinkIR: The University of Louisville's Institutional Repository. It has been accepted for inclusion in Electronic Theses and Dissertations by an authorized administrator of ThinkIR: The University of Louisville's Institutional Repository. This title appears here courtesy of the author, who has retained all other copyrights. For more information, please contact thinkir@louisville.edu. 


\title{
STUDIES OF THE EFFECTS OF PANCREATIC BETA CELL ANTIOXIDANT TRANSGENES ON EXPERIMENTAL MODELS OF DIABETES
}

\author{
By \\ Hainan Chen \\ B.S., Shanghai Medical University, 1992 \\ M.S., University of Louisville, 2002

\begin{abstract}
A Dissertation
Submitted to the Faculty of the

Graduate School of the University of Louisville

in Partial Fulfillment of the Requirements

for the Degree of
\end{abstract} \\ Doctor of Philosophy \\ Department of Pharmacology and Toxicology \\ University of Louisville \\ Louisville, Kentucky
}

December 2003 


\title{
STUDIES OF THE EFFECTS OF PANCREATIC BETA CELL ANTIOXIDANT TRANSGENES ON EXPERIMENTAL MODELS OF DIABETES
}

\author{
By \\ Hainan Chen \\ B.S. Shanghai Medical University, 1992 \\ M.S. University of Louisville, 2002
}

A Dissertation Approved on

August 21, 2003

By the following Dissertation Committee:

Dissertation Director 


\section{DEDICATION}

This dissertation is dedicated to my parent

who loved me, supported me and encouraged me all the time. 


\section{ACKNOWLEDGEMENTS}

My sincere thanks go to the following people for the generous help they offered in many different ways:

To my mentor, Dr. Paul N. Epstein, for his excellent guidance, encouragement, patience, and financial support. He has provided me with critical scientific input and interest, and independence throughout the course of this research.

To my committee members Drs. David W. Hein, William M. Pierce, Jr., Evelyne Gozal, and Jon Klein, for their valuable suggestion, critical evaluation of this project, and more importantly, their time.

To Dr. Edward C. Carlson, professor at the Department of Anatomy and Cell Biology, University of North Dakota, for his expert assistance in the islet electronic microscopy study.

To Dr. Lori Pellet and Mr. Jon T. Moritz, the former colleagues in Dr. Paul N Epstein's laboratory, for their wonderful works in islet immunohistochemistry study and establishment of transgenic mice.

To Dr. Liang Qiangrong, Mrs. Patricia Kralik, and many other members in Dr. Paul N. Epstein's laboratory, for their friendship, technical teaching and research discussion.

To my beloved wife and colleague, Mrs. Xiaoyan Li, for her understanding, support, patience and valuable discussion and technical assistance throughout the years.

This work was supported by grants from the American Diabetes Association and National Institutes of Health (NIH) grants RO1-DK52309 and RO1-DK58100, and was supported by a fellowship from the Center for Genetics and Molecular Medicine (CGeMM) at the University of Louisville. 


\title{
ABSTRACT \\ STUDIES OF THE EFFECTS OF PANCREATIC BETA CELL ANTIOXIDANT TRANSGENES ON EXPERIMENTAL MODELS OF DIABETES
}

\author{
Hainan Chen
}

August 21, 2003

Pancreatic beta cells are extremely vulnerable to the destruction of Reactive Oxygen Species (ROS). In both type 1 and type 2 diabetes ROS are involved in the loss of $\beta$-cells. We hypothesized that the deficiency in ROS detoxifying system of $\beta$-cells resulted in $\beta$-cell vulnerability and enhanced ROS protection would protect from diabetes. To test this hypothesis, transgenic mice with overexpression of $\beta$ cell antioxidant protein metallothionein (MT), manganese superoxide dismutase (MnSOD) and catalase were produced on an FVB background. Initial studies demonstrated that these transgenes did not affect $\beta$ cell normal function and morphology. But those transgenes significantly protected pancreatic islets or mice from ROS induced $\beta$-cell damage and diabetes. The transgenic islet cells had high efficacy in scavenging most forms of ROS, and generally survived and functioned better than control cells when exposed to various ROS insults. The transgenic mice were highly resistant to STZ induced diabetes.

However, inconsistent with studies on insulin secreting tumor cell lines in which antioxidants were shown to be protective, all these antioxidant transgenes failed to prevent cytokine toxicities in the cultured islets. We employed a speed congenic strategy to produce two lines of congenic NOD mice containing $\beta$-cell specific expression of MT and catalase. NOD mice spontaneously develop type 1 diabetes, and cyclophosphamide (CYP) injection accelerates NOD diabetes onset. But very unexpectedly, either transgene MT or catalase dramatically hastened diabetes onset in NOD mice. In addition, no antioxidant transgene was found to be able to protect from type 2 diabetes in an Agouti obese diabetic 
model. But the MT transgene also unexpectedly caused severe diabetes on a normal C57BL/KsJ mouse which can be made to develop type 2 diabetes by producing obesity and insulin resistance.

The data from this project indicate that the overexpression of antioxidant transgenes protects $\beta$ cells from ROS damaging stimuli but sensitizes $\beta$-cells to some other insults. Some of these data are at odds with the long term favored concept that antioxidant treatment is always beneficial for treatment of diabetes. These results imply that oxidative stress may not only be harmful, but it may also be necessary to activate self-protective mechanisms in the $\beta$-cells. 


\section{TABLE OF CONTENTS}

PAGE

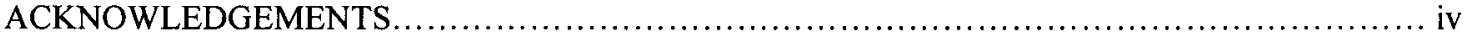

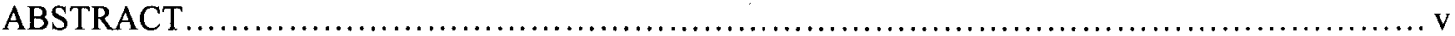

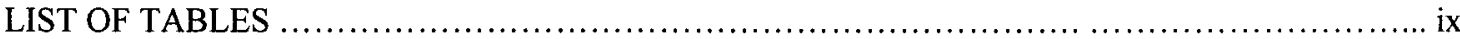

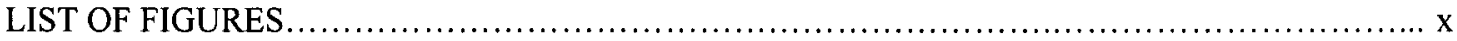

CHAPTER

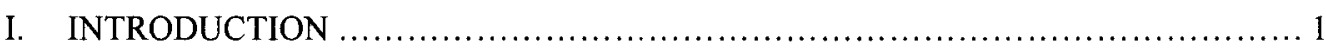

Overview............................................................... 1

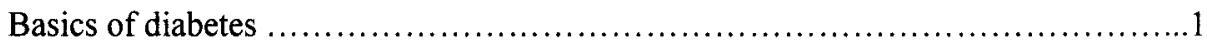

Reactive oxygen species and antioxidants ....................................

Pancreatic beta cells are extremely susceptible to ROS insults....................8

Involvement of ROS in autoimmune Type 1 diabetes.............................9

Involvement of ROS in Type 2 diabetes ..................................... 12

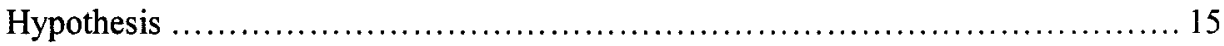

II. GENERATION AND EVALUATION OF TRANSGENIC MICE WITH PANCREATIC BETA CELL OVEREXPRESSION OF ANTIOXIDANT PROTEINS ................. 17

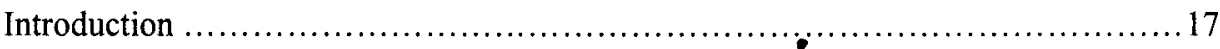

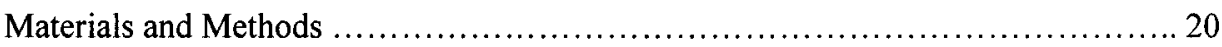

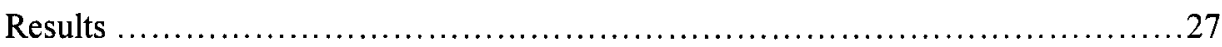

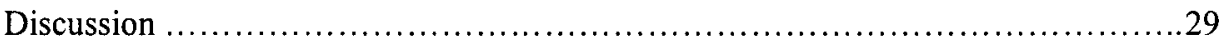

III. THE PROTECTIVE EFFECT OF ANTIOXIDANT TRANSGENES AGAINST ROS INDUCED PANCREATIC BETA CELL DESTRUCTION AND STREPTOZOTOCIN

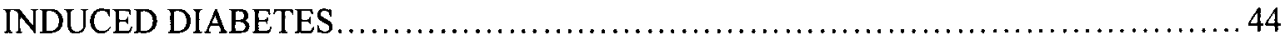

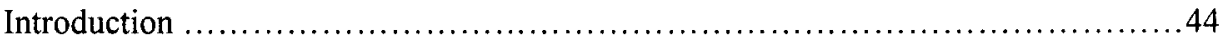


Materials and Methods

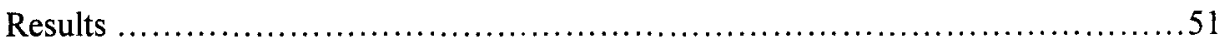

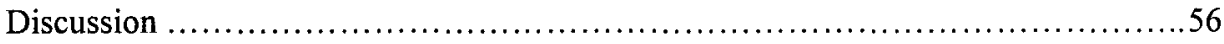

IV. THE ROLE OF ANTIOXIDANT TRANSGENES IN TOXIC EFFECT OF CYTOKINES

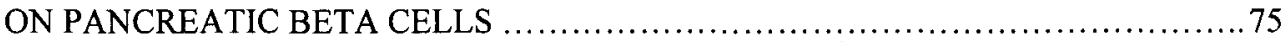

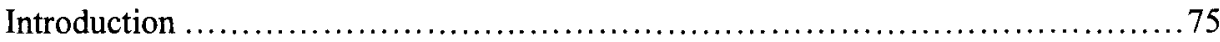

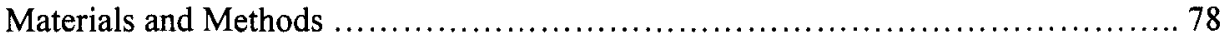

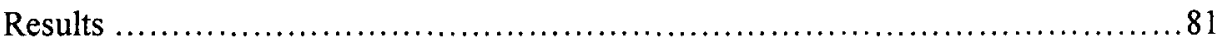

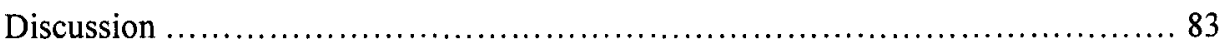

V. THE ROLE OF ANTIOXIDANT TRANSGENES IN TYPE 1 DIABETES OF NOD

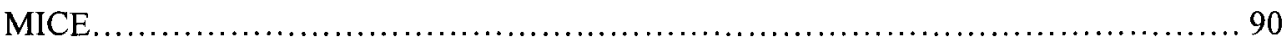

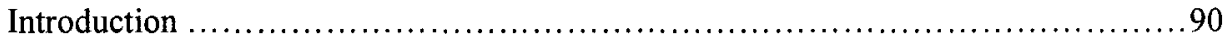

Materials and Methods ...................................................... 93

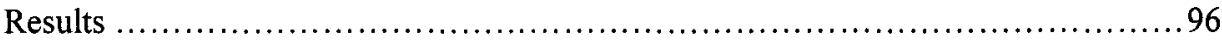

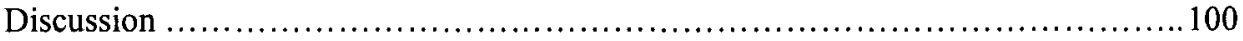

VI. THE ROLE OF ANTIOXIDANT TRANSGENES IN AGOUTI OBESE DIABETIC

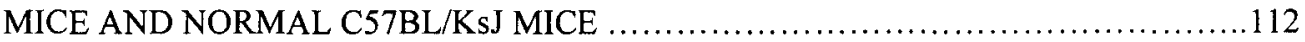

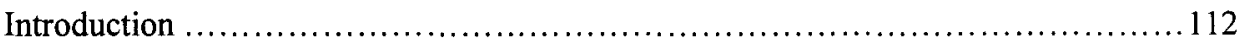

Materials and Methods ........................................................ 116

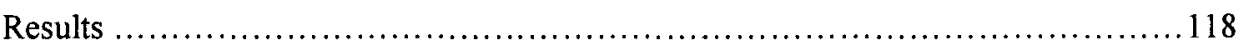

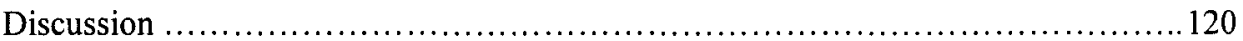

VII. SUMMARY AND DIRECTION FOR FUTURE STUDIES $\ldots \ldots \ldots \ldots \ldots \ldots \ldots \ldots \ldots \ldots 128$

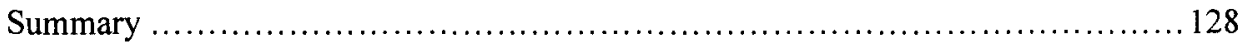

Direction for Future Studies ................................................ 131

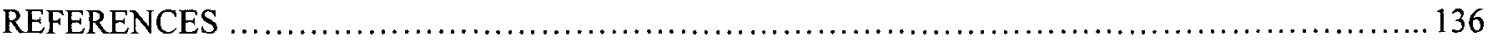

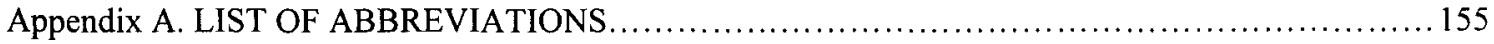

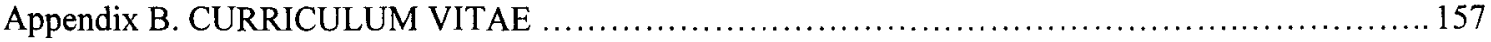




\section{LIST OF TABLES}

TABLE

2-1. Characteristics of FVB control and MT transgenic islets.

5-1. Microsatellite linkage markes for diabetic susceptible alleles (Idds) homozygous for congenic NOD mice

5-2. $\mathrm{Mg}^{2+}$ concentration for PCR reaction and approximate PCR product size for each set of microsatellite markers from NOD and FVB strains. 104

6-1. Frequency of diabetic mice in $\mathrm{C} 57 \mathrm{BL} / \mathrm{KsJ}$ mice at each generation due to the beta cell MT transgene. 


\section{LIST OF FIGURES}

Figure

PAGE

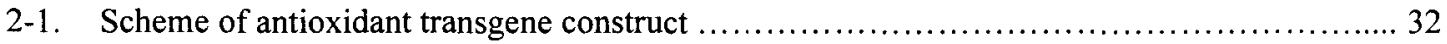

2-2. Elevated MT expression in isolated islets of HMT-1 and HMT-2 transgenic mice...............33

2-3. Increased MT immunostaining and normal insulin staining in HMT-1 islets................... 34

2-4. Glucose tolerance test in MT transgenic and control FVB mice.............................. 36

2-5. SOD activity in isolated islets from five lines of MnSOD transgenic mice and control FVB mice.

2-6. Elevated SOD immunostaining in MnSOD transgenic islets compared to FVB control islets....38

2-7. Increased catalase activity in isolated islets of catalase transgenic mice. .39

2-8. PCR identification of MnSOD and catalase transgenes in offspring from cross-breeding of MnSOD and catalase transgenic mice.

2-9. Normal insulin immunostaining and islet morphology in catalase and MnSOD

transgenic islets

2-10. Basal (3mM glucose) and $20 \mathrm{mM}$ glucose stimulated insulin secretion in MnSOD, catalase, MnSOD plus catalase transgenic islets and control FVB islets....

2-11. Glucose tolerance test in MnSOD, catalase, MnSOD plus catalase transgenic and control FVB mice.

3-1. Superoxide radical in dispersed MnSOD, catalase, MnSOD plus catalase transgenic and control FVB islet cells

3-2. ROS production in dispersed MnSOD, catalase, MnSOD plus catalase transgenic and control FVB islet cells after treatment with SIN-1

3-3. ROS production in dispersed MnSOD, catalase, MnSOD plus catalase transgenic and control FVB islet cells after treatment with $\mathrm{H}_{2} \mathrm{O}_{2}$ 
3-4. Cell viability in MnSOD, catalase, MnSOD plus catalase transgenic islets and control FVB islets after treatment with $\mathrm{H}_{2} \mathrm{O}_{2}$ for 12 hours.

3-5. ROS production in dispersed FVB control and transgenic HMT-1 islet cells.

3-6. Nitric oxide induced damage in FVB and HMT-1 transgenic islets exposed to SNAP for 24 hours 66

3-7. Synergistic protection by MnSOD plus catalase against STZ induced islet cell death and insulin loss in vitro.

3-8. Synergistic protection by MnSOD plus catalase against STZ induced diabetes. 68

3-9. Nitrotyrosine staining in pancreatic islets from $\mathrm{STZ}$ treated animals 69

3-10. Photomicrographs of FVB and HMT-1 islets after STZ treatment in vitro 70

3-11. Fragmentation of high-molecular weight DNA by STZ treatment in FVB and HMT-1 islets.... 71

3-12. NAD ${ }^{+}$levels in FVB and HMT-1 islets exposed to STZ in vitro...... 72

3-13. Blood glucose values measured after STZ treatment of FVB, HMT-1, and HMT-2 mice. 73

3-14. Transmission electron micrographs of isolated pancreatic FVB control and HMT-1 transgenic islets before and after STZ treatment.

4-1. Glucose stimulated insulin secretion and cell viability in control FVB and transgenic islets after cytokine treatment.

4-2. Inducible nitric oxide synthase expression in transgenic and control islets after treatment with cytokines

4-3. Nitric oxide release in transgenic and control islets after treatment with cytokines for 24 hours.

4-4. Glucose stimulated insulin secretion in FVB control and HMT-1 transgenic islets after cytokine treatment.

4-5. Nitric oxide release in HMT-1 transgenic and control FVB islets after treatment with cytokines.

5-1. PCR-based genotyping of diabetic susceptible alleles in congenic NOD mice with beta cell specific expression of MT transgene.....

5-2. MT staining and insulitis in NOD and MTNOD congenic mice at the age of 150 days..... 106 
5-3. Cumulative diabetes incidence in MT transgenic and nontransgenic NOD mice injected with CYP

5-4. Preserved pancreatic insulin levels in MT transgenic and nontransgenic NOD mice 8 days after CYP administration. 108

5-5. No incidence of diabetes in FVB control and HMT-1 mice after CYP administration 109

5-6. Protective effect of MT transgene against CYP mediated cytotoxicity to cultured islets from the FVB strain

5-7. Acceleration of diabetes by beta cell specific catalase transgene in CYP injected NOD mice... 111

6-1. Body weight and blood glucose level in male F1 control and Agouti mice.

6-2. Blood glycohemoglobin content in transgenic and nontransgenic F1 Agouti mice.

6-3. Blood glucose level in male F1 control and Agouti mice containing or not containing the MT transgene.

6-4. Frequency of diabetes in MT transgenic and nontransgenic C57BL/KsJ mice. 126

6-5. Morphology of pancreatic islets in MT transgenic (MTC57BLKs/J) and nontransgenic C57BLKs/J mice. 127 


\section{CHAPTER I \\ INTRODUCTION}

\section{Overview}

Diabetes is one of the most prevalent chronic diseases in the world. It is a disease characterized by high level of blood glucose that results from insufficiency of insulin secretion, or insulin action. Up to now the etiology of diabetes has not been clearly defined. Previous studies indicate that diabetes is accompanied by increased production of oxidative stress and shortage of antioxidant defenses in pancreatic beta cells. In the following text the relevance between diabetes and oxidative stress in pancreatic beta cells will be discussed. The overall objective of this research, stated in greater detail at the end of this introduction, is to test whether overproduction of reactive oxygen species (ROS) contributes to beta cell death and diabetes, and to test whether increased beta cell ROS scavengers could protect from diabetes. To achieve this goal, transgenic mouse models with beta cell specific expression of antioxidants catalase, manganese superoxide dismutase or metallothionein were utilized in this study.

\section{Basics of Diabetes}

Diabetes mellitus, which literally means "sweet excessive urine", is a chronic disease first recognized by ancient Egyptians almost 2,100 years ago. But until 1922 when Frederick Banting and Charles Best discovered insulin (Banting \& Best 1990), no significant progress was made in understanding and treating diabetes. Diabetes is a group of metabolic disorders with the common characteristic of hyperglycemia, a high level of blood glucose. This hyperglycemia results from a deficiency of pancreatic insulin secretion, or resistance to insulin action, or both. The chronic state of hyperglycemia often leads to a series of late complications affecting various organs, including kidney, heart, retina, vascular endothelium, as well as peripheral nerves. In clinics, diabetic patients are diagnosed when they have symptoms of hyperglycemia 
(typically, thirst, polyuria, weight loss, etc), and a plasma glucose level equal to or higher than 11.1 $\mathrm{mmol} / \mathrm{L}$ or a fasting plasma glucose level of at least $7.0 \mathrm{mmol} / \mathrm{L}$ (Zimmet et al. 2001).

Diabetes is one of the most prevalent chronic diseases in the world. Currently about 150 million of the global population is suffering from diabetes. $90 \%$ of them are Type 2 diabetes. The World Health Organization predicts that this number will increase to 220 million in 2010 , and 300 million in 2025 (Zimmet et al. 2001). Most of the new cases will be of Type 2 diabetes because current human environment, human behavior and lifestyle result in more obese people. This explosive increase of diabetic patients is taking place in both developed and developing countries. In the United States about 8 million people have been diagnosed with diabetes and 7 million people may have diabetes but they themselves do not know it. Diabetes has become one of the main threats to human health and imposes a huge burden on health-care systems. Diabetes is the sixth-leading cause of death by disease in the United States. Unlike cancer, stroke, and cardiovascular disease, whose death rates declined, the death rate of diabetes increased about $30 \%$ in the past 12 years. On average the United States spends about 105 billion dollars every year in treatment of diabetes or in diabetes related health care (Olefsky 2001).

Diabetes is generally classified into two major forms: Type 1 diabetes (also called insulin-dependent diabetes mellitus [IDDM]) and Type 2 diabetes (also called non-insulin dependent diabetes mellitus [NIDDM]). Type 1 diabetes is primarily due to autoimmune-mediated destruction of pancreatic beta cells, leading to absolute insulin deficiency. Type 1 diabetes usually occurs in children and young adults. Those patients would develop diabetic ketoacidosis if not treated with exogenous insulin (Eisenbarth et al. 2003). Type 2 diabetes is characterized by insulin resistance and abnormal beta cell insulin secretion. Most cases of Type 2 diabetes are obesity-associated. Patients with Type 2 diabetes, at least initially and often throughout their life, do not have a need for exogenous insulin therapy. However, if the blood glucose is not in good control insulin injection might be helpful or required. Type 2 diabetes can often be treated with diet control, exercise and oral hypoglycaemic medicines (Buse et al. 2003). 
Unfortunately, until now there is still no cure for diabetes despite the many therapies that have been tried. Although it has been known that both genetic and environmental factors are involved, the real cause for diabetes is still a mystery. The autoimmune destruction to pancreatic beta cells in Type 1 diabetes may be triggered by an interaction between environmental factors (such as a viral infection (Niklasson et al. 2003; Foulis 1996)) and an inherited genetic predisposition of the subjects. In the human genome about 20 different genetic alleles have been found to have some degree of linkage with Type 1 diabetes (Hawa et al. 2002). But the precise mechanism for how the genetic and environmental factors interact is not completely elucidated. Type 2 diabetes is a heterogeneous, polygenic disorder. More than one gene is always involved in an individual patient. But the association between these "identified" genes and Type 2 diabetes is weak and not always reproducible (Gloyn 2003). The most important environmental factor for Type 2 diabetes is obesity, which is an important acquired factor contributing to the development of insulin resistance. However the role of many genetic and environmental factors in Type 2 diabetes is not so well defined. Recently, extensive studies demonstrated that oxidative stress was significantly increased in both Type 1 and Type 2 diabetes. Pancreatic beta cells are extremely sensitive to reactive oxygen species (ROS) induced damage because beta cells contain relatively low level of ROS detoxifying systems. Therefore we propose that ROS probably are important mediators causing pancreatic beta cell dysfunction and destruction during diabetes development.

\section{Reactive oxygen species and Antioxidants}

Reactive oxygen species (ROS) and reactive nitrogen species (RNS) are oxygen or nitrogencontaining molecules that have a higher chemical reactivity than ground-state molecular oxygen or nitrogen (For brevity, in the following text the term ROS is used to indicate both ROS and RNS, unless stated otherwise). ROS include radicals such as superoxide $\left(\mathrm{O}^{{ }^{\circ-}}\right)$, hydroxyl radical $\left(\mathrm{HO} \mathrm{O}^{\circ}\right)$, nitric oxide ( $\mathrm{NO}$ ), and non-radical species such as hydrogen peroxide $\left(\mathrm{H}_{2} \mathrm{O}_{2}\right)$, and peroxynitrite $\left(\mathrm{ONOO}^{-}\right)$. Some of these species, like $\mathrm{O}^{\circ-}$ and $\mathrm{HO}^{\circ}$, are extremely unstable, while others, like $\mathrm{H}_{2} \mathrm{O}_{2}$, are relatively long-lived.

It has been known for a long time that some chemical and physical agents are able to produce ROS. For example UV light and X-rays induce ROS formation that results in cell damage (Ogawa et al. 2003). 
In biological systems, ROS are generated during the courses of normal aerobic respiration (Lenaz et al. 2002) and normal cellular activities, for example leukocyte mediated cellular disposal processes generate $\mathrm{O}^{*-}$ and $\mathrm{H}_{2} \mathrm{O}_{2}$ (Iles \& Forman 2002). Three main sites of $\mathrm{O}^{*-}$ production have been identified: the mitochondria, cytochromes on the endoplasmic reticulum, and oxidases located in the cytoplasm and on the membranes. In the mitochondrial electron transport chain, the NADH dehydrogenase component of complex I and the ubiquinone Q-cytochrome b component of complex III are believed to be the main sites of $\mathrm{O} 2^{*-}$ generation (Freeman \& Crapo 1982). When electrons are passed along the complexes of the mitochondrial electron transport chain 1-3\% (Nohl \& Jordan 1986) of electrons "leak" from these components. Rather than being passed along to the next component of the respiratory chain, some of these leaked electrons are passed to molecular oxygen to generate $\mathrm{O}^{\circ-}$. The higher the rate of metabolism is, the greater the production of $\mathrm{O}^{\circ-}$. A similar phenomenon occurs within the endoplasmic reticulum. Here, electrons are "leaked" from the NADPH cytochrome $P_{450}$ oxidoreductase (Aust et al. 1972) and from cytochrome $b_{5}$ reductase (Estabrook et al. 1979). A number of cytoplasmic and cell membrane oxidases are also capable of generating O2*- Xanthine oxidase (Nishino 1994) and NADPH oxidase (Rossi 1986) are two of the most significant cytoplasmic and membrane sources of $\mathrm{O}^{\circ-}$. NADPH oxidase, first discovered in the neutrophil, is particularly important in the "oxidative burst" of phagocytic leukocytes (Iles \& Forman 2002).

Hydrogen peroxide generation is closely linked to the sites of $\mathrm{O}^{\circ-}$ production in the cells. $\mathrm{H}_{2} \mathrm{O}_{2}$ may be produced directly from cytoplasmic oxidases such as xanthine oxidase, or from peroxisomal oxidases (del Rio et al. 1992), or indirectly as a consequence of the enzymatic dismutation of $\mathrm{O}^{\circ-}$ by superoxide dismutase. $\mathrm{H}_{2} \mathrm{O}_{2}$ itself is weakly reactive. But in the presence of transition metals such as $\mathrm{Fe}^{2+}$ and $\mathrm{Cu}^{2+}$, $\mathrm{H}_{2} \mathrm{O}_{2}$ can generate hydroxyl radical (HO) through Fenton or Haber-Weiss reaction. Iron is present in biological systems mostly as an essential part of proteins such as hemoglobin, ferritin, cytochromes, Fe-S proteins, etc. Hydroxyl radical can rapidly react with any molecules next to it to generate other free radicals (Harel \& Kanner 1988). 
Nitric oxide ( $\mathrm{NO}$ ) is one of the most important RNS produced under both physiological and pathological conditions. In eukaryotic cells, nitric oxide is synthesized from L-arginine and molecular oxygen by one of three nitric oxide synthases (NOS) (Groves \& Wang 2000) . Nitric oxide can interact with other ROS to yield a variety of highly reactive intermediates, including peroxynitrite (Koppenol et al. 1992). Peroxynitrite is a detrimental oxidant produced in activated macrophages, neutriphils and endothelial cells by a reaction between nitric oxide ( $\mathrm{NO}$ ) and superoxide (O2*-) (Bartosz 1996; Koppenol et al. 1992). The overall pathway of ROS production in the living cells is summarized as follows in Scheme 1.

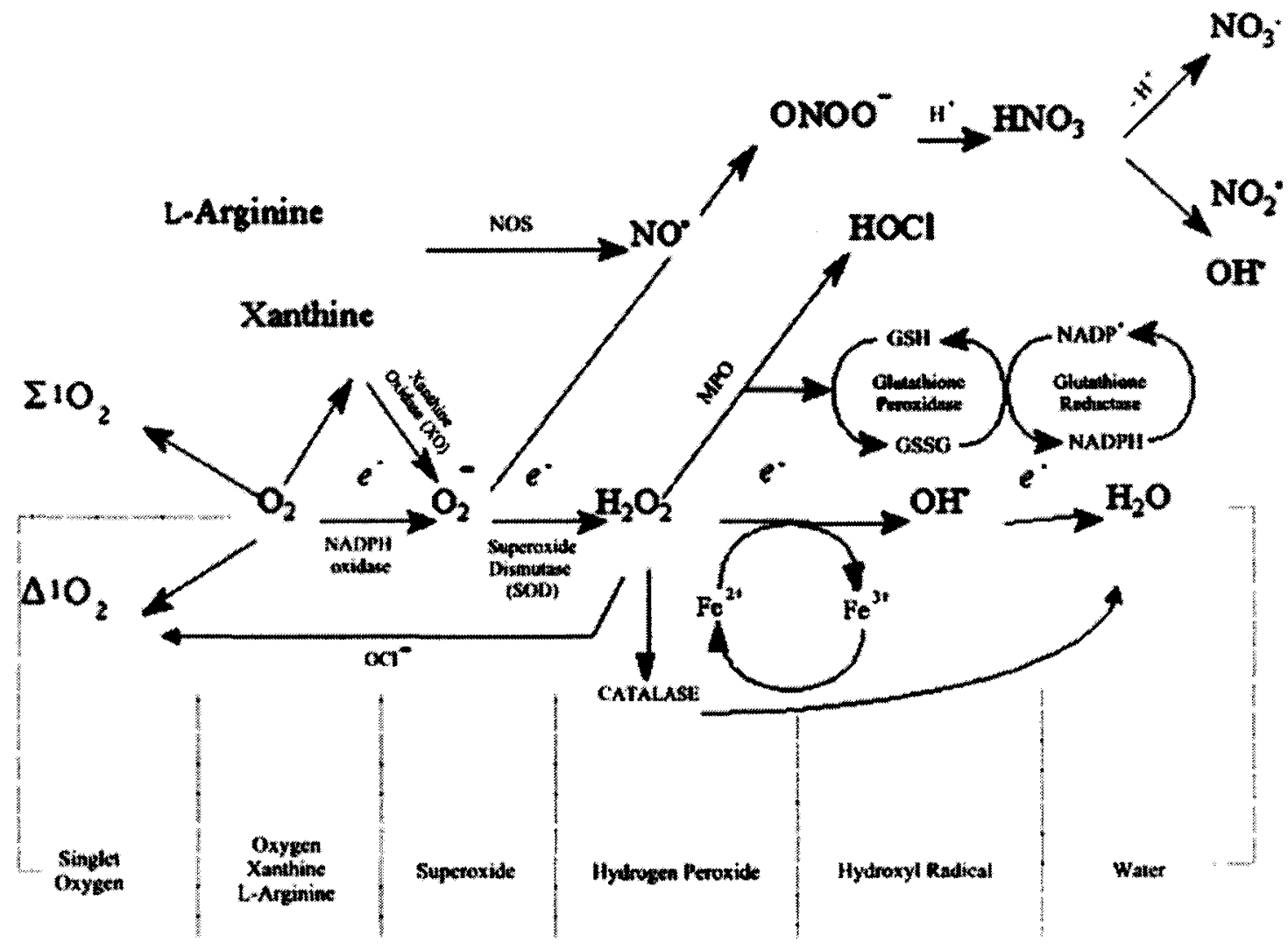

Scheme 1. Overall pathway of ROS production and enzymatic inactivation.

Despite their essential physiological function in cell signaling, cell proliferation and cellular disposal, ROS are well known to be toxic and detrimental to cells and tissues. Under certain forms of stress, such as 
UV and virus infection, increased levels of ROS are generated in the cell. Because of their high reactivity, high level of ROS can induce damage through direct reactions with numerous types of biological molecules, including proteins, lipids, DNA, RNA, steroids, etc. ROS oxidize proteins causing enzymatic inactivition (Dmitriev 2001), oxidize lipids leading to cell membrane disintegration (Benderitter et al. 2003), and oxidize DNA resulting in DNA mutation and chromosomal aberrations (Brooks 1997; Dandona et al. 1996). In addition to their ability to directly inflict macromolecular damage, ROS can function as signaling molecules to activate a number of cellular sensitive pathways whose activation can also lead to cellular damage (Forman et al. 2002): In mammalian cells, high levels of ROS mediate the inflammatory response (Wiseman \& Halliwell 1996; McCord 1993). Much evidence indicates that ROS can act as a mediator of apoptosis (Buttke \& Sandstrom 1994; Jacobson 1996). Excess of ROS may lead to cell degeneration and death (Aust et al. 1993).

In order to protect against ROS damage to cells, nature has evolved several enzymes and antioxidants that act in a combined fashion to lower the amount of ROS and ROS induced oxidative damage in the cell. These antioxidants include a number of small molecular-weight agents, such as glutathione, ascorbic acid, uric acid, vitamin E, ubiquinones, carotenoids, metallothionein, and others (Aust et al. 1993). Glutathione (Griffith 1986) is one of the most abundant reductants and acts as a thiol donor converting disulfides to thiols. It works as a cofactor for glutathione peroxidase (GPx) and glutathione S-transferase. Vitamin E and ubiquinones exist mainly in biomembranes and function as radical scavengers. Ascorbic acid is also an abundant biological reductant; it recycles vitamin $\mathrm{E}$ in scavenging lipid peroxidation. Uric acid and carotenoids are presumed to behave as singlet oxygen quenchers and radical scavengers. Metallothionein is a small protein able to dispose of many ROS including superoxide, hydrogen peroxide, nitric oxide and peroxynitrite (Cai et al. 2000; Kennedy et al. 1993; Kumari et al. 1998; Sato \& Bremner 1993).

The major antioxidant enzymes are superoxide dismutases (SODs), catalase (Cat), peroxidases (such as glutathione peroxidase (GPx) and thioredoxin peroxidase (TPx)). SODs (McCord 2002) convert superoxide to hydrogen peroxide and oxygen. At least three types of SOD have been identified in eukaryotic cells. Manganese-containing superoxide dismutase (MnSOD) is an $88 \mathrm{kDa}$ tetrameric protein 
that is primarily localized in the mitochondria matrix. MnSOD plays a key role in scavenging superoxide generated from the electron transport chain. Copper- and zinc-containing SOD ( $\mathrm{Cu} / \mathrm{ZnSOD}$ ) is a $32 \mathrm{kDa}$ dimeric protein that is localized in the cytoplasm. $\mathrm{Cu} / \mathrm{ZnSOD}$ is thought to remove superoxide generated by endoplasmic reticulum and cytosolic oxidases. The extracellular SOD (ECSOD) is a $135 \mathrm{kDa}$ tetrameric protein found in the extracellular space. ECSOD may be important in removing membrane-related oxidasegenerated superoxide. Cat and GPxs convert hydrogen peroxide to water. Cat (Deisseroth \& Dounce 1970) is found in peroxisomes or cytosol. It is a tetrameric protein with large molecular weight of $220 \mathrm{kDa}$. The GPxs (Brigelius-Flohe 1999) are a family of selenium-dependent enzymes with a unique t-RNA that incorporates one atom of selenium per subunit. At least four isoforms of GPx have been described: cytosolic GPx (GPx1), plasma GPx, phospholipid-hydroperoxide GPx (PHGPx) and gastrointestinal GPx (GPx-GI). Normal function of all GPx enzymes requires glutathione (GSH) as a cofactor, and secondary enzymes that include glutathione reductase and glucose-6-phosphate dehydrogenase (G-6-PDH). G-6-PDH generates NADPH in the pentose phosphate shunt needed by GSH recycling.

When the cells are exposed to different types of stressors, many of those antioxidant enzymes are highly induced. For examples, metallothionein content in liver, kidney, pancreas and gut can be increased several fold by interleukin-1 $\beta$ during inflammation (Courtade et al. 1998) or after exposure to exogenous heavy metals (Ohly \& Gleichmann 1995; Zimny et al. 1993). SODs and GPx are highly inducible proteins in a variety of cell types when they are challenged with cytokines (Borg et al. 1992; Strandell et al. 1995), UV light (Takada et al. 2002), or hypoxia (Yamashita et al. 1994). The increased levels of antioxidants make the cells more capable of scavenging ROS, thereby diminishing cellular oxidative damage. However, antioxidant systems are not always enough to scavenge all types of ROS. Under certain circumstance, ROS detoxifying systems are relatively deficient and oxidative damage occurs, causing cell death and disease. It has been widely accepted that ROS are involved in many types of disease or biological degeneration process, such as ischemia/reperfusion injury, cancer, arthritis, and ageing (reviewed in (McCord 1993)). Diabetes is one of the diseases highly relevant to ROS. Several recent studies found that ROS probably made a significant contribution to the beta cell death in diabetes (Ihara et al. 1999; Grankvist et al. 1981a). Following evidence supports this proposition. 


\section{Pancreatic beta cells are extremely susceptible to ROS insults}

Compared to other tissues, pancreatic beta cells are extremely vulnerable to the destruction caused by ROS. This sensitivity has been demonstrated in many in vitro studies exposing pancreatic islets or beta cell lines directly to ROS. Xanthine oxidase generated superoxide anion (Burkart et al. 1992), nitroprusside generated nitric oxide (Eizirik et al. 1994a), or hydrogen peroxide (Takasu et al. 1991) inhibit beta cell insulin secretion, decrease mitochondrial respiration, fragment beta cell DNA and induce beta cell death. Two widely used diabeteogenic chemicals, alloxan and streptozocin, specifically damage pancreatic beta cells and induce experimental diabetes in animals (Grankvist et al. 1981a). The action of both compounds is mediated by ROS including hydrogen peroxide (Takasu et al. 1991), superoxide radicals (Nukatsuka et al. 1988) and nitric oxide radicals (O'Neill et al. 1993). Some xenobiotic chemicals also cause diabetes in humans. Intoxication with the rodenticide Vacor (Esposti et al. 1996), an inhibitor of NADH:ubiquinone reductase, results in an acute human diabetes. This is probably due to the toxicity of reducing electrons leaking from the mitochondrial respiratory chain. More than other tissues, pancreatic islets are readily destroyed by ROS. Cultured rat islets are 2.5 to 25 times more sensitive to peroxide radicals than muscle, kidney and liver (Malaisse et al. 1982). Beta cell derived HIT and RIN cell lines have much less capacity to degrade hydrogen peroxide than macrophage like cell lines J-774 cells (Zhang et al. 1995).

This unique sensitivity of beta cell to ROS could be explained either by insufficient ROS detoxifying systems in beta cells or by presence of a mechanism that allows these ROS related toxins to attack beta cells more effectively. The beta cell glucose transporter, GLUT2 (Schnedl et al. 1994) is thought to contribute to the specific toxicity of STZ to beta cells because it can transport STZ. However, many other tissues, such as liver and kidney that are relatively resistant to STZ, also express GLUT2. In vitro no ROS accumulation in islet cells was found when islets were exposed to hydrogen peroxide or peroxide radicals (Zhang et al. 1995; Malaisse et al. 1982). In contrast, the ROS relatively resistant tissues, liver and kidney contain much higher levels of protective ROS scavengers than beta cells (Grankvist et al. 1981b). Therefore it is logical to extrapolate that a relative deficit of ROS protective systems leads to the striking sensitivity of beta cells to the destruction by ROS. 
Direct analysis of ROS scavengers reveals that pancreatic beta cells are short of ROS detoxifying systems. The level of three most important enzymatic antioxidants, superoxide dismutases (SODs), glutathione peroxidase, and catalase is extremely low in beta cells. Grankvist et al (Grankvist et al. 1981b) reported that total islet SOD level was less than $30 \%$ of that measured in liver. The levels of glutathione peroxidase and catalase in islets were as low as less than $2 \%$ of liver and among the lowest of all tissues assayed (Malaisse et al. 1982; Grankvist et al. 1981b; Johansson \& Borg 1988; Welsh et al. 1995). Therefore the inherent inadequacy of ROS detoxifying systems in beta cells makes themselves particularly vulnerable to ROS damage. Any elevation of antioxidant levels specifically in beta cells is projected to provide protection against ROS destruction to the pancreatic beta cells.

\section{Involvement of ROS in autoimmune Type I diabetes}

Type 1 diabetes is an autoimmune disease characterized by the selective destruction of beta cells within the islets of Langerhans (Bach 1994). This destruction is a complex process involving beta cell antigen autoantibodies generated by B lymphocytes, inflammatory cell infiltration (termed insulitis) and cytokine release (Eizirik et al. 1996; Bach 1994; Oberley 1988; Horio et al. 1994). ROS participate in these processes leading to beta cell destruction through the oxidation of nucleic acids, proteins and membrane lipids (Gutteridge \& Halliwell 1990; Wiseman \& Halliwell 1996; Halliwell \& Gutteridge 1995).

During autoimmune attack, ROS are generated by activated macrophages (Nathan 1987) and by beta cells themselves in response to cytokines (Rabinovitch et al. 1992). The cytotoxic effect of activated marcophage is thought to initiate early damage on beta cells through the action of ROS. In non-obese diabetic (NOD) mouse model of Type 1 diabetes, hydrogen peroxide was found markedly increased in isolated islets co-cultured with macrophage-enriched fractions (Horio et al. 1994). The antioxidant probucol can reverse the elevation of hydrogen peroxide, thereby providing a significant protection against cyclophosphamide speeded diabetes in NOD mice (Fukuda et al. 1995). Upregulation of ROS release is also found in macrophages from autoimmune diabetes prone BB rats (Brenner et al. 1993). Apart from direct cytotoxicity by macrophages and T-lymphocytes (Nagata \& Yoon 1992), cytokines secreted from 
these cells such as interleukin 1-beta (IL-1 $\beta$ ), tumor necrosis factor-alpha (TNF- $\alpha$ ) and interferon-gamma (IFN- $\gamma$ ), cause a broad spectrum of damaging events ranging from an inhibition of beta cell function up to beta cell death through necrosis and apoptosis (Eizirik et al. 1996; Rabinovitch \& Suarez-Pinzon 1998). ROS are also involved in these destructive events. Reactive oxygen intermediates were indicated to be produced in both cultured human islets (Rabinovitch et al. 1996) and rat islets (Rabinovitch et al. 1992) after treatment with combined cytokines IL-1 $\beta$, TNF- $\alpha$ and IFN- $\gamma$. Nitric oxide (NO), which is generated in beta cells through the activation of inducible NO synthase (iNOS) gene, is one of the most important mediators for cytokine induced beta cell toxicity (Corbett et al. 1993; Flodstrom et al. 1999). NO can react with superoxide to produce highly reactive and toxic peroxynitrite that is found in beta cells of diabetic NOD mice (Suarez-Pinzon et al. 2001; Suarez-Pinzon et al. 1997). Mice whose iNOS gene has been knocked out are highly resistant to autoimmune diabetes generated by multiple low doses of streptozotocin (Flodstrom et al. 1999).

Since ROS are so essential in autoimmune diabetes, many trials using antioxidants to treat diabetes and prevent beta cell damage have been performed. Non-enzymatic chemicals with antioxidant properties, including vitamin E (Behrens et al. 1986), probucol (Shimizu et al. 1991; Drash et al. 1988), MDL 29,311 (Heineke et al. 1993), and lazaroid (Rabinovitch et al. 1993), have been tested in various rodent Type 1 diabetes models including the NOD mouse, the Bio-Breeding (BB) rat, and the low dose STZ model. The results revealed that antioxidant treatment was beneficial to preventing diabetes. More current studies were carried out to examine the potential role of antioxidant proteins SOD, catalase and glutathione peroxidase. These enzymes were either administrated using a carrier or overexpressed in the beta cell lines by gene transfer technologies. Horio et al (Horio et al. 1994) reported that in vivo administration of catalase or SOD coupled to polyethylene glycol reduced islet damage in the NOD models of diabetes. In insulinproducing tumor cell lines catalase and glutathione peroxidase have been shown to have protective effects. Overexpression of catalase or glutathione peroxidase in beta cell-like RINm5F cells provided protection from hydrogen peroxide, superoxide anion and NO (Tiedge et al. 1997). But the effect of SOD is inconsistent. In one beta cell line, INS-1 cells, MnSOD overexpression completely protected from IL-1 $\beta$ 
mediated cytotoxicity through the inhibition of iNOS activation (Hohmeier et al. 1998). However, single overexpression of $\mathrm{Cu} / \mathrm{Zn}$ SOD in RINm5F cells either had no effect on the toxicity of hydrogen peroxide or even increased the toxicity, while co-expression of SOD and catalase provided protective effect on RINm5F cells (Tiedge et al. 1998). This indicates that SOD action may differ in different cellular situations and its proper action may need catalase complementation. A recent study by Lortz et al (Lortz et al. 2000) reported that overexpression of antioxidant proteins $\mathrm{Cu} / \mathrm{Zn} \mathrm{SOD}$, catalase and glutathione peroxidase in RINm5F cells significantly increased the resistance to cytokines through inactivation of ROS generation rather than through reduction of NO generation. Moreover, a recent in vivo study by Hotta et al (Hotta et al. 1998) strongly supported antioxidant therapy benefited NOD diabetes. In their study an antioxidant protein thioredoxin was overexpressed specifically in beta cells of NOD mouse. With this overexpression, the incidence of diabetes in NOD mice was markedly reduced. Therefore, these data, particularly in cultured beta cell lines suggest an important role of ROS contributing to beta cell death during Type 1 diabetes and a potential therapeutic value of antioxidants in treatment of Type 1 diabetes.

However some studies failed to observe any protective effect by ROS scavengers. Burkhart (Burkart \& Kolb 1993) et al reported that SOD, catalase, and antioxidant compounds citiolone and dimethylthiourea did not improve the viability of cultured islets damaged by activated macrophage. Welsh et al (Welsh et al. 1994c) tested liposome delivered SOD, catalase and gluthathione peroxidase against IL-1 $\beta$ induced beta cell toxicity. Their results were also negative. Hence, uncertainty about the role of ROS in Type 1 diabetes still remains. But unlike the study of Hotta et al. (Hotta et al. 1998) where thioredoxin was uniquely expressed in beta cells at a higher level in a permanent fashion, the antioxidant application assisted by glycerol or liposome is neither permanent nor targeted to the beta cells. The success of thioredoxin implies that antioxidant treatment against Type 1 diabetes may require an early startup and a specific action on beta cells for a long time. On the other hand, the conclusion from thioredoxin study can still be criticized in that thioredoxin has other functions other than being an antioxidant (Hirota et al. 1999; Nakamura et al. 1997). Therefore in this project we used more specific antioxidants MnSOD and catalase to investigate the role of ROS in Type 1 diabetes. 


\section{Involvement of ROS in Type 2 diabetes}

Type 2 diabetes is characterized by both insulin resistance and pancreatic beta cell dysfunction. During the development of Type 2 diabetes, the normal insulin secretion of beta cells is impaired and beta cells may eventually undergo degeneration. Recent evidence suggests that ROS play an important pathological role in degeneration of the beta cells in Type II diabetes. The presence of ROS leads to abnormal cell function or cell death because ROS oxidize cellular DNA, protein and lipid to nonfunctional derivates such as 8-hydroxydeoxyguanosine (8-OHDG), 3-nitrotyrosine, malondialdehyde (MDA) (reviewed in (Rosen et al. 2001). These oxidized products have been widely used as biomarkers to indicate the oxidative damage caused by ROS. It has been demonstrated that these biomarkers are significantly enhanced in urine (Dandona et al. 1996) and plasma samples (Gopaul et al. 1995) from Type 2 diabetes patients. Direct evidence showing oxidative damage present in beta cells comes from an immunohistochemistry studies (Ihara et al. 1999) in Goto-Kakizaki (GK) rat, a model of Type 2 diabetes. In this diabetic rat the beta cells had very high levels of 8-OHDG and 4-hydroxy-nonenal (HNE) modified proteins, while in nondiabetic control animals these two markers were virtually undetectable. The level of oxidative damage indicated by these two markers corresponded to the severity of diabetes. Supplementation of antioxidant vitamin E to GK rats revealed an accumulation of vitamin $\mathrm{E}$ in pancreas and improvements of glucose tolerance and diabetic control (Ihara et al. 2000).

The source of oxidative stress to the beta cells in Type 2 diabetes is not as clear as that in Type 1 diabetes. Chronic hyperglycemia or long-chain fatty acid deposition in beta cells may both be involved in ROS generation (Hayden \& Tyagi 2002). Under diabetic conditions with high concentrations of glucose, ROS release and resultant beta cell destruction have been proven in a variety of studies. Kaneto et al (Kaneto et al. 1996) reported that culture of a beta cell line, HIT-T15 cells, in high glucose medium raised cellular levels of hydrogen peroxide and malondialdehyde. When antioxidant $\mathrm{N}$-acetyl-L-cysteine (NAC) or aminoguanidine (AG) was added to the culture medium, the high glucose induced decrements of insulin mRNA, insulin gene promoter activity, DNA binding activity of insulin promoter transcription factors PDX-1 and RIPE-3b1, as well as the loss of insulin content and secretion in HIT-T15 cells were all significantly reduced (Tanaka et al. 1999a). In vivo application of these two antioxidants to Zucker 
diabetic fatty rats, a model of Type 2 diabetes, reduced the elevation of oxidative stress markers, improved insulin secretion and prevented the development of glucose intolerance (Tanaka et al. 1999a). In another Type 2 diabetes animal model, $\mathrm{db} / \mathrm{db}$ mice, chronic treatment with $\mathrm{NAC}$ or antioxidant vitamins $\mathrm{E}$ or $\mathrm{C}$ reduced beta cell mass loss, maintained beta cell insulin content and improved glucose tolerance (Kaneto et al. 1996).

The mechanism of ROS generation in beta cells exposed to high glucose is still not completely elucidated. Many studies have emphasized the essential roles of ROS in the development of diabetic complications in heart, neurons and kidney after long term exposure to hyperglycemia (Nishikawa et al. 2000). The mechanism for hyperglycemia induced ROS production in tissues other than pancreatic islets may also apply to beta cells. Hyperglycemia can increase the levels of ROS through non-enzymatic glycosylation, glucose autoxidation and the polyol pathway (Nishikawa et al. 2000). In non-enzymatic glycosylation, glucose initially undergoes a nucleophilic addition reaction with proteins to form a Schiff base, forming early glycosylation products that are reversible when blood glucose level returns to normal. However, the early products may form Amadori compounds, a process that can form $\mathrm{H}_{2} \mathrm{O}_{2}$ in vitro. Amadori products can generate $\mathrm{H}_{2} \mathrm{O}_{2}$ via two pathways. One pathway is the 1,2-enolization pathway, which generates $\mathrm{H}_{2} \mathrm{O}_{2}$ and glucosone under anaerobic conditions. The other pathway, the main pathway to produce $\mathrm{H}_{2} \mathrm{O}_{2}$ under oxidative conditions, is 2,3-enolization pathway that forms 1-deoxyglucosone and the putative 1,4-deoxyglucosone. Further reactions of Amadori compounds result in polymerization of proteins by forming insoluble, irreversible crosslinking complexes called advanced glycosylation end-products (AGEs). AGEs tend to accumulate on long-lived macromolecules in tissues, leading to abnormalities of cell and tissue functions.

In addition to direct glycosylation reactions, monosaccharides and fructose-lysine can spontaneously reduce molecular oxygen under physiological conditions. This process is called glucose autoxidation which is able to produce oxidizing intermediates, such as $\mathrm{O}_{2}{ }^{--}, \mathrm{OH}$ and $\mathrm{H}_{2} \mathrm{O}_{2}$, and alpha-ketoaldeydes. Those ROS can not only damage lipids and proteins, but also accelerate the formation of AGEs, which in turn 
produce more ROS. Some metal chelating agents that prevent autoxidation have been shown to protect from experimental diabetes in animals (Nakamura et al. 2002).

ROS can also be generated via polyol/sorbitol pathway. Exposure to elevated glucose levels increase intracellular sorbitol and fructose content due to aldose reductase and sorbitol dehydrogenase activity. Oxidation of sorbitol to fructose is coupled to reduction of NADP ${ }^{+}$to NADPH at the expense of NADH. An increase in $\mathrm{NAD}^{+} / \mathrm{NADH}$ ratio is linked to $\mathrm{O}_{2}{ }^{--}$formation via the reduction of prostaglandin $\mathrm{G} 2$ $\left(\mathrm{PGG}_{2}\right)$ to prostaglandin $\mathrm{H}_{2}\left(\mathrm{PGH}_{2}\right)$ by prostaglandin hydroperoxidase (Minegishi et al. 2002).

Hyperglycemia and hyperlipidemia usually occur together in Type 2 diabetes. Elevated free fatty acids (FFAs) not only induce a state of insulin resistance (Paolisso et al. 1995), but also impair beta cell function (Bollheimer et al. 1998; Zhou \& Grill 1994). There is also evidence that FFAs may cause beta cell death (Lee et al. 1997; Shimabukuro et al. 1998a). The toxicity of FFAs may be mediated by nitric oxide and/or ceramide. Shimabukuro et al (Shimabukuro et al. 1997) demonstrated that FFAs significantly induced NO production through the activation of iNOS gene in cultured islets from ZDF rats. Treatment with antioxidants nicotinamide or aminoguaunidine improved the insulin secretion that was inhibited by FFAs. In vivo both compounds had the effects on preventing beta cell destruction and hyperglycemia. Shimabukuro et al also reported that apoptosis was increased in islets of ZDF rat (Shimabukuro et al. 1998a) accompanied by an excessive production of NO and ceramide (Shimabukuro et al. 1998b; Shimabukuro et al. 1998a). The elevated ceramide results from de novo synthesis from unoxidized FFA deposits in beta cells. ROS may be released from mitochondria upon ceramide action, in that ceramide can regulate the permeability of the mitochrondial membrane and activity of respiratory complex III (reviewed in (Mathias et al. 1998)). A recent study (Listenberger et al. 2001) supported a direct involvement of ROS in lipotoxic response although it did not involve ceramide. In this study Chinese hamster ovary (CHO) cells were exposed to saturated fatty acid palmitate. It was found that palmitate induced apoptosis through the generation of ROS detected by an oxidant-sensitive fluorescent probe. Antioxidants pyrolidine dithiocarbamate and 4,5-dihydroxy-1,3-benzene-disulfonic acid both blocked palmitate-induced caspase activation and DNA laddering. However, another recent report totally excluded NO or other ROS effects 
on beta cell death caused by FFAs (Cnop et al. 2001). When isolated normal Wistar rat islets were exposed to increasing concentrations of palmitate or oleate, both apoptosis and necrosis were induced. The cytotoxicity of FFAs was dose dependent. Nevertheless, neither iNOS mRNA increase nor NO formation was detectable and therefore did not contribute to the FFA toxicity. The application of antioxidants including nicotinamide, SOD or mixtures of vitamin A, C, E, dithiothreitol and glutathione could not reverse the cytotoxicity of FFAs. In contrast to the previous studies using diabetic or prediabetic ZDF rats or their islets, the islets used in that study are normal Wistar rat islets. Normal islets may behave differently in response to FFAs since they probably have sufficient and/or normal cellular mechanisms to prevent FFA induced cell death. All these result indicate that ROS may contribute to the beta cell destruction in Type 2 diabetes, however the roles and mechanisms of ROS in Type 2 diabetes warrant further investigation.

\section{$\underline{\text { Hvpotheses }}$}

Substantial evidence supports the concept that pancreatic beta cells are extremely vulnerable to ROS damage and ROS are involved in both Type 1 and Type 2 diabetes. Therefore, we hypothesize that the deficit of ROS detoxifying systems in pancreatic beta cells significantly contributes to beta cell pathology and that enhanced ROS protection will reduce beta cell destruction caused by ROS and thereby prevent experimental diabetes.

Despite extensive studies, it is still uncertain what roles ROS play in the diabetes because most of the evidence can be criticized for limitations in experimental approach: (1) Most ROS scavengers that have been used have actions other than ROS protection. For example, the antioxidant probucol inhibits macrophage function in addition to being an antioxidant (Kita et al. 1987). The most promising agent thioredoxin used in the study by Hotta et al (Hotta et al. 1998) could have additional effects such as antiapoptosis rather than antioxidant only. (2) Enzymatic antioxidants, such as catalase and SOD, do not enter the beta cells as freely as small molecule chemical antioxidants. Consequently exogenous administration of these antioxidant proteins usually has low efficacy. (3) In vitro studies oversimplify diabetes and may not extrapolate to the whole animals. (4) Apart from the action on beta cells, ROS scavengers applied in vivo 
may have actions on other sites such as the immune system, liver or lipid metabolism, or other systems (Jialal et al. 2002; Venugopal et al. 2002), which confounds the interpretation of the results. (5) Also, in some situations the application of ROS scavengers in vivo does not always prevent diabetes. Eliminating these limitations as much as possible is necessary for a clear test of the role of ROS in diabetes. To exclude these uncertainties in this study we used transgenic mouse models. In these transgenic animals, three different types of antioxidant proteins catalase, MnSOD or MT were expressed uniquely in the beta cell at high and stable levels starting at the birth of animals. These antioxidant proteins are able to scavenge many types of ROS. Hence this in vivo system provided an optimal model for investigation of the ROS hypothesis.

The following specific aims were carried in this study to test this hypothesis.

(1) Determined whether elevated catalase, MnSOD, MnSOD plus catalase or MT provided protection against beta cell toxins. Transgenic islets or mice were tested for resistance to streptozotocin, superoxide anion, hydrogen peroxide, peroxynitrite and nitric oxide.

(2) Determined whether these elevated antioxidant proteins provided protection against immune attack related to Type 1 diabetes. Transgenes were tested for protective effects against cytotoxicity in islets exposed to combined interleukin- $1 \beta$ and interferon- $\gamma$, and against diabetes in the NOD mouse model of Type 1 diabetes.

(3) Determined whether these elevated antioxidant proteins prevented oxidative damage and preserved beta cell function in states of Type 2 diabetes. Transgenes were tested for protective effects in obese agouti mice, a model of Type 2 diabetes. 


\section{CHAPTER II}

\section{GENERATION AND EVALUATION OF TRANSGENIC MICE WITH PANCREATIC BETA CELL SPECIFIC OVEREXPRESSION OF ANTIOXIDANT PROTEINS}

\section{Introduction}

The first successful transgenic animal was a transgenic mouse created in 1980 (Gordon et al. 1980). Since then, huge amounts of transgenic animals have been generated with incredible speed of advancement for biomedical research. But to date transgenic mice are still the most popular animals in transgenic research. Several factors may account for this popularity. One reason is that the mouse is easy to manipulate and maintain in the laboratory. The other reason is that the genetic structure and physiological systems of mouse and human are quite similar. Scientists already have a solid understanding of the mouse genome, embryology and physiology in that mouse has been used as an animal model for biomedical research for more than 100 years. Therefore the generation and manipulation of transgenic mice for researchers is readily accessible; the assessment and interpretation of data from transgenic mice is easier to obtain and as informative, accurate and reliable as that from rats and other types of animals (Boyd \& Samid 1993; Hammer et al. 1985).

A transgenic animal is an animal whose genome has been artificially modified in order to study the potential function of the modified gene (or genes) of interest. The creation of transgenic animals involves a transfer of a genetically engineered segment of foreign gene (or genes) into the genetic material of the animal. This introduced foreign gene is called transgene. When the transgene is integrated into the host genome, it is stable to be transmitted through the germ line to the offspring. Currently there are at least three basic methods to produce transgenic animals, which are DNA microinjection, retrovirus mediated gene transfer and embryonic stem cell mediated gene targeting. In this project we produced transgenic animals by DNA microinjection procedure. In this method the transgene was first constructed in a genetic 
carrier (commonly a plasmid) by aligning a segment of DNA sequence (gene of interest) within several regulatory elements, including tissue specific promoter, enhancer and poly A tail. The constructed transgene was then microinjected with a very fine needle into the pronucleus of a one cell mouse embryo. These microinjected embryos were implanted into the oviduct of pseudopregnant mice. Progenitor mice were born with the transgene three weeks later. Then the transgenic mice were identified and maintained by subsequent cross-breeding (Gordon 1993).

Nowadays, transgenic animals have become a very valuable tool for biomedical research and pharmaceutical product development. They have made a great contribution to research for human welfare, such as xenotransplantation (Zhou et al. 2002; Vial et al. 2000), supplementation of nutrition (Wheeler et al. 2001) and pharmaceutical products (Kadokawa et al. 2003). But more commonly the transgenic animals are utilized as animal models for human diseases, thereby helping scientists in discovering etiology and pathology of the diseases, and improving the therapeutics of those diseases (Yamamura et al. 1992; Ishiguro et al. 2001). The advantage of using transgenic animals in this field is clear-cut. Since the transgene is integrated into host genome, the expression of the transgene is stable and heritable. This long term and stable expression is clearly suitable for many chronic disease studies. Since the transgene is expressed in a truly in vivo state, the interaction of transgene with proteins, hormones, neurotransmitters, and other components of the animals provides a much more complete and physiologically relevant picture of the transgene's function than could be achieved in any other way, for example in cultured cells. With tissue specific promoters, transgenes may be specifically expressed in certain types of cells or organs. This confined expression pattern allows a clear-cut test of the potential of the transgene in a specific organ or a specific biological process. The economic value of transgenic animal is also significant. Compared with some other classic research techniques, using transgenic animals often significantly reduces the number of required animals and the time for studies (Contrera \& DeGeorge 1998; Jegstrup et al. 2003).

Many transgenic mice with beta cell specific expression of foreign genes have been produced. In those animals pancreatic beta cells have overexpressed genes for anti-apoptotic proteins (Liu et al. 1996), growth factors (Hoeflich et al. 1999), transcription factors (Welsh et al. 2002; Flodstrom \& Eizirik 1997; 
Dutta et al. 2001), ion channels (Koster et al. 2000), or metabolic enzymes (Epstein et al. 1992), etc. These transgenic animals have provided a profound knowledge about beta cell development, physiology, and diabetes pathology and therapeutics. However few transgenic mice with beta cell overexpression of antioxidant transgenes have been generated. Apart from the models in our laboratory, $\mathrm{Cu} / \mathrm{Zn}$ SOD has been expressed in beta cells and shown to protect from alloxan induced diabetes (Kubisch et al. 1997). Thioredoxin, which actually has multiple functions; including being an anti-apoptotic protein and an antioxidant protein, was found to prevent NOD diabetes and STZ induced diabetes by specific expression in pancreatic beta cells (Hotta et al. 1998). But transgenic overexpression of extracellular SOD in beta cells failed to prevent NOD diabetes (Sandstrom et al, 2002). The concept that antioxidants are effective to protect beta cell against damage in diabetes is mostly based on in vitro studies carried on insulin secreting cell lines and isolated islets (Tiedge et al. 1998; Tiedge et al. 1999; Hohmeier et al. 1998). Therefore the proof of this concept awaits further in vivo evidence.

To carry out these in vivo studies, we produced lines of transgenic FVB mice with elevated expression of antioxidant proteins: rat catalase, human MnSOD, and human MT. These antioxidants were expressed specifically in pancreatic beta cells regulated by the insulin promoter. Both Type 1 (NOD mice) and Type 2 diabetes (Agouti mice) animal models with beta cell specific expression of those antioxidants were also developed by continuous back-crossing or cross-breeding. By using those transgenic mice we have tested whether direct antioxidant protection of pancreatic beta cells provides protection against oxidative damage to pancreatic beta cells and against Type 1 and Type 2 diabetes. 


\section{Materials and Methods}

Chemicals: Collagenase (type V), Histopaque 1077, purified bovine erythrocyte SOD and rat liver metallothionein were obtained from Sigma (St. Louis, MO). Hank's balanced salt solution (HBSS), RPMI 1640 medium, fetal bovine serum (FBS) and PCR reaction reagents (including Platinum Taq DNA polymerase, dNTP, Tris PCR buffer and $\mathrm{MgCl}_{2}$ ) were supplied by Invitrogen (Carlsbad, CA). Proteinase $\mathrm{K}$ was purchased from Qiagen (Valencia, CA) and ${ }^{109}$ cadmium was from Amersham (Piscataway, NJ). Rat insulin standard was bought from Linco (St. Charles, MO). Rabbit antiserum to guinea pig insulin, was purchased from BioGenex (San Ramon, CA). Mouse anti-horse MT antibody was supplied by Dako (Carpinteria, CA). Sheep anti-human SOD antibody was bought from Biodesign International (Saco, Maine). For construction of the transgene enzymes were obtained from New England Biolabs (Beverly, MA). Other non-indicated reagents were standard chemicals from Sigma (St. Louis, MO), Fisher Scientific (Pittsburgh, PA) or Amersham Biosciences (Piscataway, NJ).

Solutions: The following solutions, very commonly used in this project, will not be further described wherever they were used. They are islet culture medium (with or without phenol red) (RPMI 1640 medium supplemented with $10 \%$ fetal bovine serum plus $100 \mathrm{U} / \mathrm{ml}$ penicillin and $100 \mu \mathrm{g} / \mathrm{ml}$ streptomycin), modified KRB buffer (Krebs-Ringer bicarbonate buffer, containing the designated concentration of glucose and supplemented with $0.1 \%$ BSA and $10 \mathrm{mM}$ Hepes, pH 7.4), islet sonication buffer (10mM Tris/HCl, pH 7.0, 1mM EDTA, 1mg/ml RIA grade BSA,), acid ethanol for islet insulin extraction (75 ethanol / 2 concentrated $\left.(12 \mathrm{M}) \mathrm{HCl} / 23 \mathrm{H}_{2} \mathrm{O}, \mathrm{v} / \mathrm{v} / \mathrm{v}\right)$, islet DNA digestion buffer $(0.1 \mathrm{M}$ Tris/HCl, pH 8.5, $5 \mathrm{mM}$ EDTA, $0.2 \%$ SDS, $200 \mu \mathrm{g} / \mathrm{ml}$ proteinase $\mathrm{K}$ ), Tris-acetate-EDTA (TAE) buffer for electrophoresis (0.04 M Tris/HCl, 0.001 M EDTA, pH 8.5).

Animals: The transgenic mice were generated on FVB strain which was purchased from Jackson Laboratory (Bar Harbor, Maine). Transgenic mice were maintained by cross-breeding with plain FVB mice. The presence of transgenes was determined by PCR of mouse tail DNA and/or by observation of coat color which was a product from a co-injected second transgene tyrosinase. All mice were housed in ventilating cages at the University of Louisville, Reseach Resource Center with free access to tap water and 
standard mouse chow. All animal procedures were approved by the Institutional Animal Care and Use Committee. The laboratory was certified by the American Association of Accreditation for Laboratory Animal Care.

Transgene constructs and establishment of transgenic mice: Figure 2-1 is a simplified scheme showing the construct of our antioxidant transgenes. The antioxidant gene was regulated with a rat or human insulin promoter, which allowed the antioxidant protein to express specifically in pancreatic beta cells. An SV-40 enhancer sequence was placed in the downstream portion of the antioxidant gene for an efficient expression in the target tissues. These transgenes were constructed in specific plasmids as described below. To generate transgenic mice, the transgenes were removed from the plasmid sequences by cutting with specific restriction enzymes and microinjected into FVB mouse embryos. A second transgene containing a cDNA for the enzyme tyrosinase was co-injected with the antioxidant transgene. The enzyme tyrosinase produces coat color pigmentation in albino mice (Brooks 1997) such as FVB and NOD mice. This pigmentation helps to conveniently identify transgenic animals. The embryos injected with transgenes were transferred back into the womb of pseudopregnant FVB female mice to produce offspring. From those offspring, the transgenic progenitors were established by determining the presence of the transgenes using PCR of the tail DNA. Cross-breeding between these progenitors and plain FVB mice was carried out to maintain and expand the colonies. Transgenic mice were further assessed by immunohistochemistry and antioxidant protein activity assays for assessing the expression activity of transgenes.

In more detail, the MT transgene, designated HMT, was constructed utilizing the plasmid INS.HBS provided by Dr. Timothy Stewart (Genentech, California). This plasmid contained the human insulin promoter and first intron followed by unique BamH I and Hind III sites. A $2.4 \mathrm{~kb}$ Nco I / Hind III fragment containing all introns and exons of the human MT II gene was ligated behind the insulin promoter utilizing the BamH I and Hind III sites of INS.HBS. Prior to ligation the Nco I and BamH I sites were blunt ended with Klenow polymerase. Before microinjection, the $4100 \mathrm{bp}$ HMT transgene was removed from plasmid sequences by cutting with Hind III and EcoRI. 
The catalase transgene, designated Cat, was constructed in a plamsid pKS/RIP provided by Dr. Fatima Bosch (Barcelona, Spain) as described previously (Xu et al. 1999). This gene contained the rat insulin I promoter linked to $1.8 \mathrm{~kb} \mathrm{BamHI} / \mathrm{Xho}$ I fragment of the rabbit beta globin gene that included the last beta globin intron and the globin polyadenylation sequence. An SV-40 enhancer sequence was contained in the downstream portion of the gene. The catalase cDNA was inserted into a unique Eco RI site of pKS/RIP located $45 \mathrm{bp}$ downstream from the rabbit beta globin intron. The rat catalase cDNA contained the entire $1581 \mathrm{bp}$ catalse coding sequence and was cleaved from the transgene MyCat (Kang et al. 1996) with Sal I and Hind III. The termini of the catalase fragment were converted to Eco RI sites by blunt ending and ligation to Eco RI linkers. The $4250 \mathrm{bp}$ transgene was removed from the plasmid sequences by cutting with Xho I and Sac I. The MnSOD transgene, designated as MnSOD, was constructed in the same plasmid pKS/RIP by replacing the catalase cDNA with a full length human MnSOD cDNA.

PCR typing of transgenes: To determine the presence of transgenes, mouse tail DNA was extracted with Qiagen DNAeasy kit (Qiagen, Valencia, CA) according to the manufacturer instructional manual. PCR reaction was performed in a $20 \mu \mathrm{l}$ volume containing $1.5 \mu \mathrm{l}$ sample DNA (about $20 \mathrm{ng}$ DNA), 200 $\mu \mathrm{M} d N T P, 1 \mu \mathrm{M}$ of each primer, 0.8 unit of Platinum Taq DNA polymerase, $2 \mathrm{mM} \mathrm{MgCl}_{2}$ and $10 \mathrm{mM}$ Tris $1 \times$ PCR buffer (Invitrogen, Carlsbad, CA). PCR reaction was carried out in an Eppendorf thermocycler equipped with a heated hood. The reaction contained a first polymerase activation step at $95^{\circ} \mathrm{C}$ for 2 min, followed with 35 cycles of amplification, and ended with an extension step at $72^{\circ} \mathrm{C}$ for 7 min. Each amplification cycle consisted of $1 \mathrm{~min}$ of denaturing at $94^{\circ} \mathrm{C}, 1 \mathrm{~min}$ of annealing at $57^{\circ} \mathrm{C}$ and $1 \mathrm{~min}$ of extension at $72^{\circ} \mathrm{C}$. PCR products were fractionated on $0.9 \%$ agarose gel in TAE electrophoresis buffer and stained with $0.5 \mu \mathrm{g} / \mathrm{ml}$ ethidium bromide. The sequences for each set of primers for each transgene were as follows: MnSOD (forward 5'-GGATCCTGAGAACTTCAG-3', reverse 5'-TTCTGCCTGGAGCCCAGAT AC-3'); Catalase (forward 5'-AATATCGTGGGTGACCTCAA-3', reverse 5'-GGATCCTGAGAACTTC AG-3'); MT (forward 5'-AATCGGTTGTGGACTGAGGA-3', reverse 5'-CCTGATAAGATCACTGAG GA-3'). In addition to the primers for transgene, another set of primers (forward 5'-CTGGTGATGGCTT CCTTG-3', reverse 5'- CTCAATGTTGAGCAGGAA-3') was included in every PCR reaction in order to monitor the quality of DNA sample and PCR reaction. This pair of primers was used to identify heme 
oxygenase which is ubiquitously expressed in all strains of our mice. At each time control PCR reactions for transgene positive and negative DNA were also performed.

Islet isolation: The isolation procedure was based on a modification of the method of Gotoh et al (Gotoh et al. 1985). Mice were anesthetized with avertin at the dose of $6 \mathrm{mg} / \mathrm{g}$ body weight via intraperitoneal injection. The pancreas was inflated with $3 \mathrm{ml}$ of $1.55 \mathrm{mg} / \mathrm{ml}$ collagenase Type $\mathrm{V}$, and carefully removed from the mouse body and incubated at $37^{\circ} \mathrm{C}$ for $20 \mathrm{~min}$. Digestion was stopped by adding cold HBSS followed by vigorous shaking and 3 washes in $50 \mathrm{ml}$ cold HBSS and passage through a $200 \mu \mathrm{m}$ mesh. The final pancreas pellet was resuspended in $10 \mathrm{~mL}$ Histopaque 1077 , overlaid with $10 \mathrm{ml}$ of HBSS and centrifuged at $700 \times \mathrm{g}$ for $20 \mathrm{~min}$ at $10^{\circ} \mathrm{C}$. Most of the gradient, excluding the pellet was collected and washed twice with cold HBSS. Islets were then handpicked and maintained in islet culture medium at $37^{\circ} \mathrm{C}$ and $5 \% \mathrm{CO}_{2}$ plus humid air. Islets were normally be used the day after isolation. For experiments requiring extended culture, medium was changed every two days.

Immunohistochemistry for insulin, MT and MnSOD: Transgenic or control mouse pancreas was

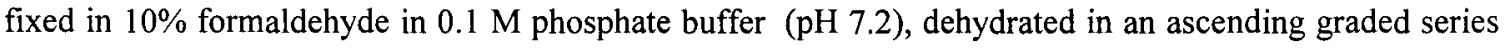
of ethanol and subsequently infiltrated with paraffin. Serial sections were cut at $5 \mu \mathrm{m}$, mounted on polylysine coated slides and then rehydrated in a descending graded series of ethanol. Slides were treated with $3 \%$ hydrogen peroxide followed by an additional 10 min incubation with Power Block (Biogenex). Sections were treated with primary antibody (rabbit anti-guinea pig insulin antibody diluted 1 to 100 or mouse anti-horse MT and sheep anti-human SOD antibodies at a 1:50 dilution). Primary antibody incubations were $90 \mathrm{~min}$ at $37^{\circ} \mathrm{C}$. After 4 washes in phosphate buffered saline slides were incubated with the appropriate biotin-labeled second antibody followed by peroxidase labeled streptavidin and developed with 3-amino-ethylcarbazone (AEC) as chromagen. On some sections the primary antibodies were omitted or replaced with non-immune sera of the appropriate species. Those sections always demonstrated negligible levels of staining. 
Measurement of islet MT content. Islet metallothionein content was determined by Cadmium-Chelex assay (Bartsch et al. 1990). After an overnight incubation, 100 to 150 islets were rinsed 3 times with HBSS and then sonicated in $150 \mu \mathrm{l} 10 \mathrm{mM}$ Tris $\mathrm{HCl}, 85 \mathrm{mM} \mathrm{NaCl}, \mathrm{pH} 7.4$ for 1 minute on ice. After 30 minutes of centrifugation at $20,000 \times \mathrm{g}$ at $4{ }^{\circ} \mathrm{C}, 100 \mu \mathrm{l}$ of the supernatant was incubated with $100 \mu \mathrm{l}$ acetonitrile for $3 \mathrm{~min}$ at room temperature. One $\mathrm{ml}$ of $10 \mathrm{mM}$ Tris $\mathrm{HCl}, 85 \mathrm{mM} \mathrm{NaCl}, \mathrm{pH} 7.4$ was added to the mixture. The solution was then incubated with $10 \mu \mathrm{l}$ of $1 \mathrm{mM} \mathrm{CdCl} \mathrm{Cl}_{2}$ containing $740 \mathrm{kBq} / \mathrm{ml}{ }^{109} \mathrm{Cd}$ for 5 min at room temperature. Chelex binding was carried out on a shaker for $15 \mathrm{~min}$ after adding $100 \mu \mathrm{l}$ Chelex suspension ( $66 \% \mathrm{v} / \mathrm{v}$ in $10 \mathrm{mM}$ Tris $\mathrm{HCl}, 85 \mathrm{mM} \mathrm{NaCl}, \mathrm{pH} 7.4)$. The resin and precipitate were removed by centrifugation at $12,000 \times \mathrm{g}$ for $5 \mathrm{~min}$, and the supernatant fraction was analyzed for ${ }^{109} \mathrm{Cd}$ with a Beckman gamma counter. Metallothionein concentrations were determined from a standard curve of purified rat liver metallothionein (Sigma). Values were expressed per $\mu$ g of islet protein in the sonicate, determined with the Pierce BCA protein assay.

Measurement of SOD activity in isolated islets. Islet SOD activity was measured by inhibition of the autooxidation of pyrogallol (Marklund \& Marklund 1974). Two hundred frozen islets were sonicated in $250 \mu \mathrm{l}$ of prechilled assay buffer $\quad(50 \mathrm{mM}$ Tris-cacodylic acid, $\mathrm{pH} \quad 8.2, \quad 1 \mathrm{mM}$ diethylenetriamineeprentaacetic acid) for $1 \mathrm{~min}$ on ice. After solubilization for $30 \mathrm{~min}$ on ice, the sonicate was centrifuged at $20,000 \times \mathrm{g}$ for $30 \mathrm{~min}$. The supernatant was used to determine islet SOD activity performed in a 96-well plate. In each well $50 \mu \mathrm{l}$ of the supernatant was mixed with $175 \mu \mathrm{l}$ of assay buffer, followed by a quick addition of $25 \mu \mathrm{l}, 2 \mathrm{mM}$ pyragallol dissolved in $10 \mathrm{mM} \mathrm{HCl}$. After reaction for $2 \mathrm{~min}$, the absorbance increasing rate of each well was read in a microplate reader at the wavelength of $405 \mathrm{~nm}$ for $2 \mathrm{~min}$ at a 10 -second interval. SOD activity was determined from a standard curve obtained with purified bovine erythrocyte SOD (Sigma). Values were expressed per mg of islet protein in the sonicate, determined with the Pierce BCA protein assay.

Measurement of catalase activity in isolated islets. The assay for islet catalase activity was carried out by Bo Xu (Xu et al. 1999) based on a peroxidatic procedure of (Johansson \& Borg 1988). Briefly 100 
islets were homogenized in $100 \mu \mathrm{l}$ of $25 \mathrm{mM} \mathrm{KH}_{2} \mathrm{PO}_{4}$, pH 7.0 by sonication. Twenty microliters of this extract was added to a reaction mixture containing $45 \%$ methanol, $0.025 \% \mathrm{H}_{2} \mathrm{O}_{2}$ as the substrate of catalase. After the reaction was stopped by $7.8 \mathrm{M} \mathrm{KOH}$, the chromogen purpald ( $34 \mathrm{mM}$, Sigma) was added to develop colorized product which was further oxidized and stabilized by addition of $\mathrm{KIO}_{4}$. The final absorbance of each vial was measured at the wavelength of $550 \mathrm{~nm}$. The islet catalase activity was calculated from a standard curve of purified bovine catalase (Sigma) and expressed as equivalent milliunits of that bovine catalase per islet.

Assays for insulin secretion, insulin content and DNA content. Glucose stimulated islet insulin secretion was measured by static assay on 96-well tissue-culture microplates. For each well 6-10 islets were hand picked into modified $\mathrm{KRB}$ buffer containing $3 \mathrm{mM}$ glucose at $37^{\circ} \mathrm{C}$. After two washes in $3 \mathrm{mM}$ glucose modified KRB solution, the islets were incubated for consecutive 30 min incubations in $200 \mu 1$ of 3 and $20 \mathrm{mM}$ glucose modified KRB solution. The supernatant was removed carefully for the measurement of released insulin. To determine islet insulin content, islets were sonicated in $150 \mu 10 \mathrm{mM}$ Tris $\mathrm{HCl}$, $1 \mathrm{mM}$ EDTA, $1 \mathrm{mg} / \mathrm{ml}$ RIA grade BSA, $\mathrm{pH} 7.0$ for 30 seconds. Fifty $\mu 1$ of the solution was used to extract islet insulin with $100 \mu \mathrm{l}$ acid ethanol at $4{ }^{\circ} \mathrm{C}$ overnight. The remainder of the sonicate was digested with an equal volume of $200 \mu \mathrm{g} / \mathrm{ml}$ proteinase $\mathrm{K}$ in $100 \mathrm{mM}$ Tris $\mathrm{HCl}$, pH 8.5, $5 \mathrm{mM}$ EDTA, $0.2 \%$ SDS at $55{ }^{\circ} \mathrm{C}$ for 2 hours and used for islet DNA quantification with picogreen kit from Molecular Probes (Eugene, OR). Insulin was measured with the coated tube RIA kit from Diagnostic Products (Los Angeles, CA) and rat insulin standards.

In vivo glucose tolerance test. Prior to the test, sex- and age-matched mice were fasted overnight (about 16 hours). On the second day, mice were injected i.p. with a sterile $10 \%$ glucose solution at a dose of $1 \mathrm{~g} / \mathrm{kg}$ body weight. Tail blood glucose of each mouse was measured by a glucometer (OneTouch Ultra, Life Scan) at the times $0,5,15,30,60,120 \mathrm{~min}$ after glucose administration. 
Data analysis. Data are presented as the mean \pm standard error. Statistical analysis was performed by one-way or two-way ANOVA and Dunnet's post hoc (2-tailed) test. Computations were carried using statistical programs from SPSS (version 10.0) and Sigmastat (version 2.03). 


\section{$\underline{\text { Results }}$}

\section{Transgenic mice with elevated MT expression in pancreatic beta cells.}

Two independent MT transgenic lines, designated HMT-1 and HMT-2, were produced in this study. The expression level of MT in pancreatic beta cells was assessed by measuring $C \mathrm{Cd}^{109}$ binding in isolated pancreatic islets. This assay is based on the fact that MT binds cadmium with very high affinity (Bartsch et al. 1990). As shown in Figure 2-2, islets of lines HMT-1 and HMT-2 contained 30 fold and 4 fold higher levels of MT, respectively, than islets of FVB. This elevated expression of MT was confirmed by immunohistochemical staining of pancreas section from line HMT-1 with MT specific antibodies (Upper panels, Figure 2-3). HMT-1 islet had enormous MT staining compared to FVB control islet whose MT expression was barely detectable. This figure also demonstrates that MT is specifically expressed in pancreatic islets in that the MT expression was almost undetectable in the nearby acinar tissues of pancreas. Similar results were obtained with line HMT-2 (data not shown).

Since several transgenes have produced unintended beta cell dysfunction (Epstein et al. 1989; Lo et al. 1988; Wogensen et al. 1994; Wogensen et al. 1994), we also characterized HMT-1 and HMT-2 islets with respect to pancreatic islet morphology and insulin staining, isolated islet insulin content, DNA content and glucose stimulated insulin secretion. The result showed that the insulin staining and islet morphology appeared normal in MT transgenic islets (Lower panels, Figure 2-3). Both HMT-1 and HMT-2 transgenic islets contained similar content of islet insulin and DNA, and responded to glucose by secreting insulin to the same extent as control FVB islets (Table 2-1). An intraperitoneal glucose tolerance test (Figure 2-4) was performed in both transgenic lines and control FVB mice. The MT transgene, either at high or low levels of expression, did not alter the response of whole animals to in vivo glucose challenge. These results demonstrated that MT overexpression was not harmful to the beta cell.

\section{Transgenic mice with beta cell specific overexpression of MnSOD and/or Catalase.}

In total, five lines of MnSOD transgenic mice, designated $\mathrm{MnSOD}_{1}, \mathrm{MnSOD}_{2}, \mathrm{MnSOD}_{3}, \mathrm{MnSOD}_{4}$, $\mathrm{MnSOD}_{5}$, were generated in this study. Three of them had elevated SOD activity in isolated pancreatic islets as measured by inhibition of the autooxidation of pyrogallol (Figure 2-5). The line $\mathrm{MnSOD}_{3}$ 
contained the highest SOD activity, about 9 fold higher than FVB control. Immunohistochemical staining with an anti-human SOD antibody verified this increased SOD activity in the pancreatic islets (Figure 2-6). Seven lines of catalase transgenic mice were produced in our laboratory. Enzymatic activity assay, performed by Dr. Bo Xu, revealed that all lines of catalase transgenic mice had increased levels of catalase expression. Line 25 had the highest catalase activity (Figure 2-7).

Since MnSOD and catalase work successively to scavenge superoxide radical and $\mathrm{H}_{2} \mathrm{O}_{2}$, the combination of MnSOD and catalase might provide more powerful protection in pancreatic beta cells. Therefore in this study the line $\mathrm{MnSOD}_{3}$ and line 25 of the catalase transgenic mice, which had the highest level of enzyme activities, were selected for cross-breeding. Four types of offspring, nontransgenic, MnSOD, catalase, and MnSOD plus catalase transgenic mice were produced from this breeding. Theoretically each of them occupies $25 \%$ of the litters. The presence of transgenes in those litters was determined by routine PCR technique as shown in Figure 2-8.

Similar to the analysis performed in MT transgenic mice, the evaluation study of beta cell function and morphology was also carried out in catalase and MnSOD transgenic mice. The insulin staining and islet morphology appeared normal in both catalase and MnSOD transgenic islets (Figure 2-9). Either transgene alone or combination of MnSOD and catalase transgenes did not change the normal insulin secretion function in isolated islets (Figure 2-10). The islet insulin content and DNA content were also similar among transgenic and control FVB islets (Data not shown). In vivo transgenic animals with beta cell expression of either transgene (catalase or MnSOD) or both behaved similarly to FVB control mice after glucose administration (Figure 2-11). These data suggested that overexpression of MnSOD or catalase or both did not impair normal pancreatic beta cell function and structure. 


\section{Discussion}

In this part of study, we produced lines of transgenic mice with beta cell specific expression of antioxidant proteins MT, catalase, MnSOD and MnSOD plus Catalase. Both antioxidant protein activity assay and immunohistochemistry revealed that the antioxidant proteins were expressed and localized in pancreatic islets. Overexpression of those antioxidants was not harmful to beta cells since the insulin content, DNA content and insulin secretion function of transgenic islets, as well as glucose tolerance response in transgenic mice were not different from control FVB islets or mice.

We were able to produce several transgenic lines for each of our transgenes. Part of this success was due to use of the FVB mouse strain. This inbred strain that has been widely used to produce transgenic mice. At each step of transgenic technique procedure, FVB mouse have been shown to have the highest efficiency compared to other strains such as Swiss Webster (SW) outbred and C57BL/6 inbred mice. FVB mice are easy to superovulate and its embryo is relative large, thereby relatively easy for manipulation of transgene microinjection. The fertilized eggs of FVB mouse survive much longer than those from C57BL/7 inbred or other strains. FVB mouse are also productive and easy to breed (Taketo et al. 1991; Auerbach et al. 2003).

Our transgenic mice were created by microinjection technique. This technique allows the foreign transgene sequence to integrate into the host genome by a DNA recombination known to be largely random (Copeland et al. 2001). Multiple insertion sites and incorrect insertion orientation may both occur. Therefore the expression levels of the same transgene in different transgenic lines could be highly variable. In this study each line had a very different expression level of antioxidant protein. A few of them even contain no increase in activity compared to the control mice although they were all shown positive for the transgene by tail DNA PCR. The low level of expression was probably due to a bad insertion site. Due to variable expression in different lines studies on multiple lines of transgenic mice are usually required (Pujol-Borrell \& Bottazzo 1988). 
However, from another point of view, the variation of transgene expression makes it possible to test the effect of protein dosage (Black, Jr. et al. 2002). Different levels of gene expression may trigger cellular process at different degrees and thus lead to different levels of biological function or phenotype. Such a study assures a more complete identification of the potential of the transgene. As seen in our MT transgenic mice, the line of HMT-1 has a higher expression of MT than the line of HMT-2. We found that the higher level of expression of MT produced more protection (shown in Figure 3-13). In this project three types of antioxidant transgenes with different ROS scavenging spectrum were selected. For each transgene at least two lines of transgenic mice with different levels of antioxidant expression were maintained. Consistent results with two different transgenic lines eliminates the possibility that insertional mutagenesis could cause observed phenotypes. Therefore the conclusions drawn from these mice should be specific and reliable.

All of our transgenes were regulated by the rat or human insulin promoters. The insulin promoter drives the antioxidant gene expression specifically in the beta cells. We have confirmed this specificity with immunohistochemical staining in pancreatic sections. However, the insulin promoter, especially the rat insulin promoter may also drive the transgene to express in other types of tissues (Sarvetnick et al. 1988). Apart from the expression in pancreatic islets, our catalase transgene has been shown to be also expressed in liver and kidney, at a very low levels (Xu et al. 1999). Such ectopic expression might be critical to interpret the resultant data under certain situations, for example in the NOD mouse. Since the beta cell destruction in NOD mouse is mediated by an immune reaction, the expression of antioxidant transgene in immune system could alter the overall effect in the transgenic mice. Unfortunately, we have not carried out studies to examine the expression pattern of MT and MnSOD transgenes in the immune system. This needs to be completed in the near future.

We analyzed the isolated islet function and structure as well as the glucose tolerance response in the transgenic mice. The results from these studies imply that our transgenic mice or beta cells are normal compared to FVB controls. These are important analyses because transgene expression may produce unintended beta cell dysfunction and cell death (Epstein et al. 1989; Lo et al. 1988; Wogensen et al. 1994). 
Several reasons may explain this unexpected effect. DNA recombination between transgene and host genome is completely random; thereby some host genes, important for beta cell function and survival, might be disrupted. The transgene itself is highly expressed which is not physiological. The overproduction of transgenic protein could be a stressful burden to beta cells (Contreras et al. 2003). For undetermined reasons some transgenes have been found to modify normal cellular physiological signal pathways and promote beta cell death (Epstein et al. 1989). However, in this research we were lucky enough to establish lines of antioxidant transgenic mice without damaging normal beta cell function and morphology. This allowed us to further investigate their potential roles in diabetes onset and development. 


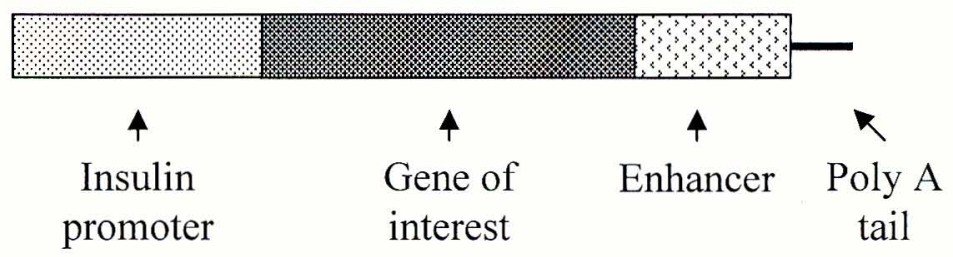

Figure 2-1. Scheme of antioxidant transgene construct. The antioxidant transgene was regulated by a rat or human insulin promoter, which allowed a specific expression in pancreatic beta cells. Following the insulin promoter was the gene or cDNA of interest containing coding sequences for rat catalase, human MnSOD or human MT-II gene. An SV-40 enhancer sequence and poly A sequence were placed in the downstream portion of the antioxidant gene for efficient expression and processing in the transgenic mice. 


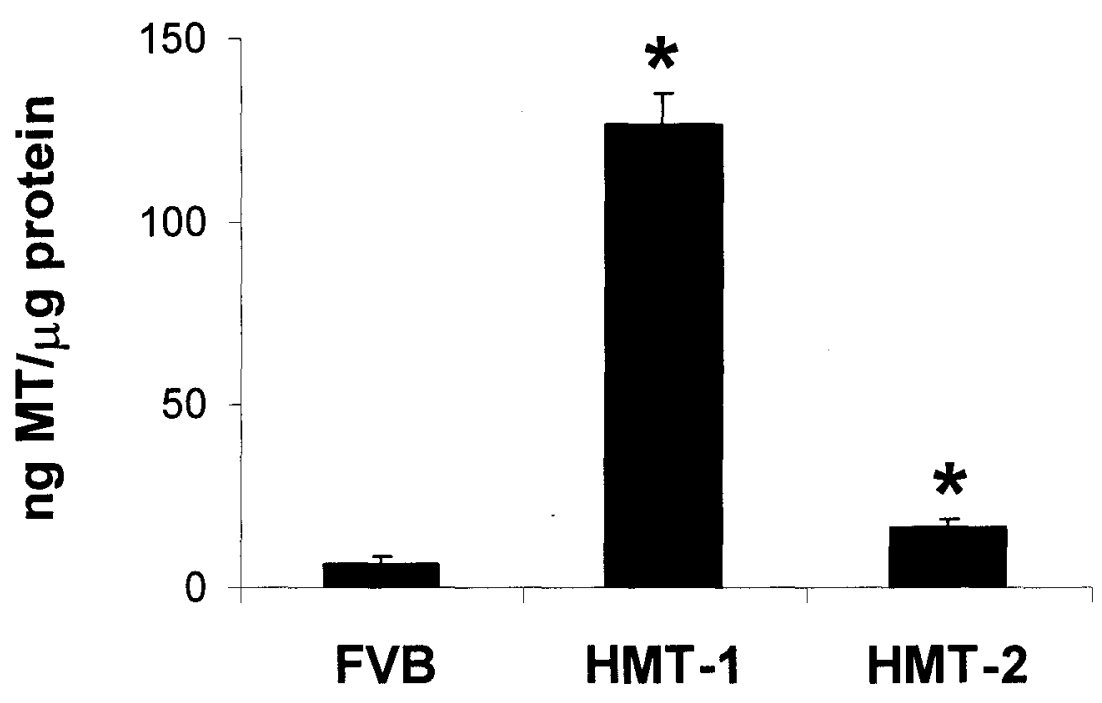

Figure 2-2. Elevated MT expression in isolated islets of HMT-1 and HMT-2 transgenic mice. The level of MT was indicated by cadmium binding activity in islet extracts as described in the methods. Four or more assays using at least 100 islets were performed for each point. An asterisk indicates different from FVB by $p<0.01$ by ANOVA and Dunnet's post hoc test. Vertical bars indicate the standard error. 

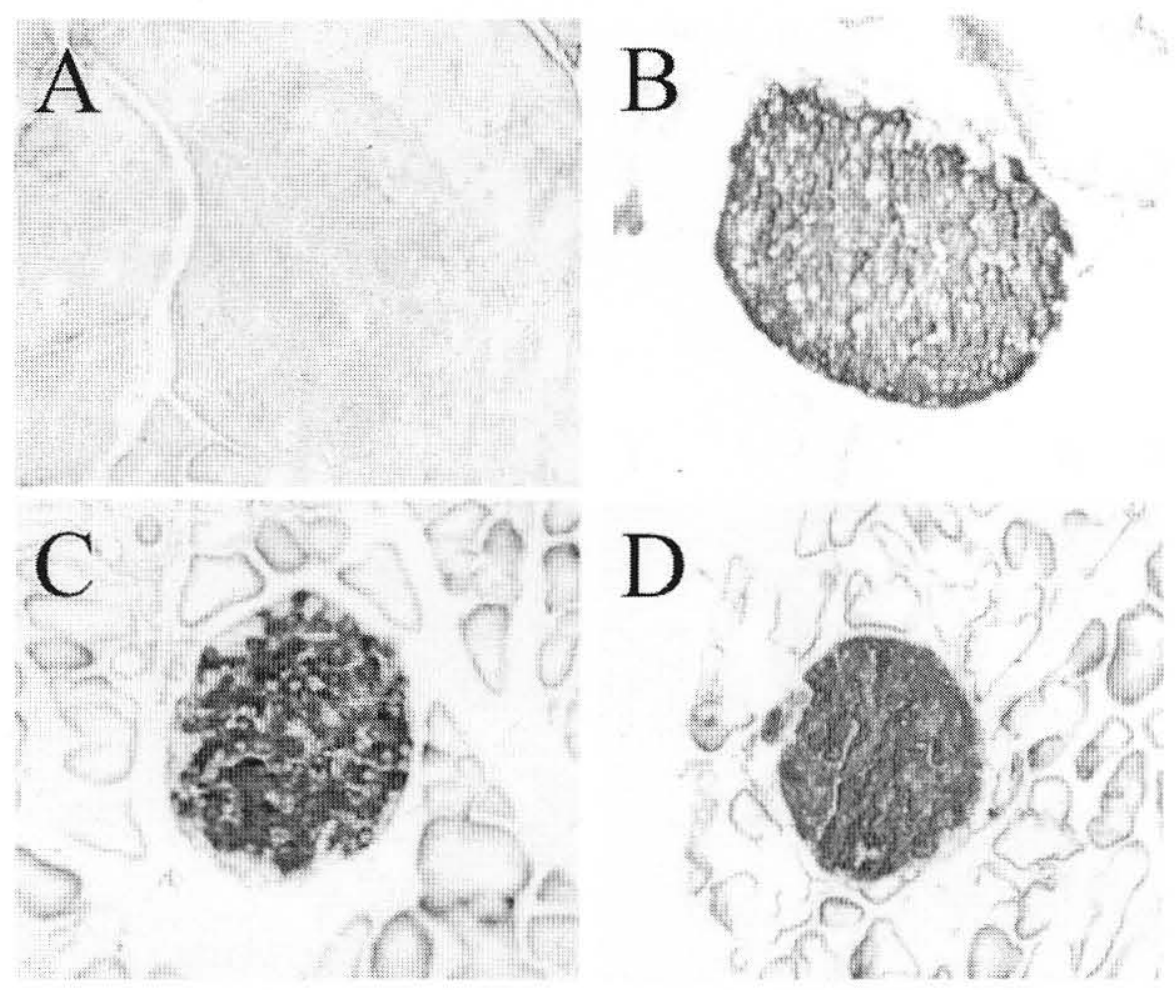

Figure 2-3. Increased MT immunostaining and normal insulin staining in HMT-1 islets. Islets in A (FVB) and B (HMT-1) were stained with a monoclonal anti-horse MT primary antibody. Islets in C (FVB) and D (HMT-1) were stained with a rabbit anti-guinea pig insulin primary antibody. Magnification $\times 200$. 
TABLE 2-1. Characteristics of FVB control and MT transgenic islets.

\begin{tabular}{cccc}
\hline & DNA (ng/islet) & Insulin ( $\mu \mathrm{U} / \mathrm{ng}$ DNA) & $\begin{array}{c}\text { Glucose induced insulin } \\
\text { secretion }\end{array}$ \\
& & & \\
FVB & $11.48 \pm 0.61$ & $190.9 \pm 7.28$ & $8.14 \pm 1.22$ \\
HMT-1 & $12.20 \pm 1.84$ & $172.5 \pm 19.9$ & $8.10 \pm 2.07$ \\
HMT-2 & $12.85 \pm 1.57$ & $161.9 \pm 11.6$ & $8.48 \pm 1.14$ \\
\hline
\end{tabular}

Data are the means \pm SE from at least 9 assays. No significant differences were found among groups. 


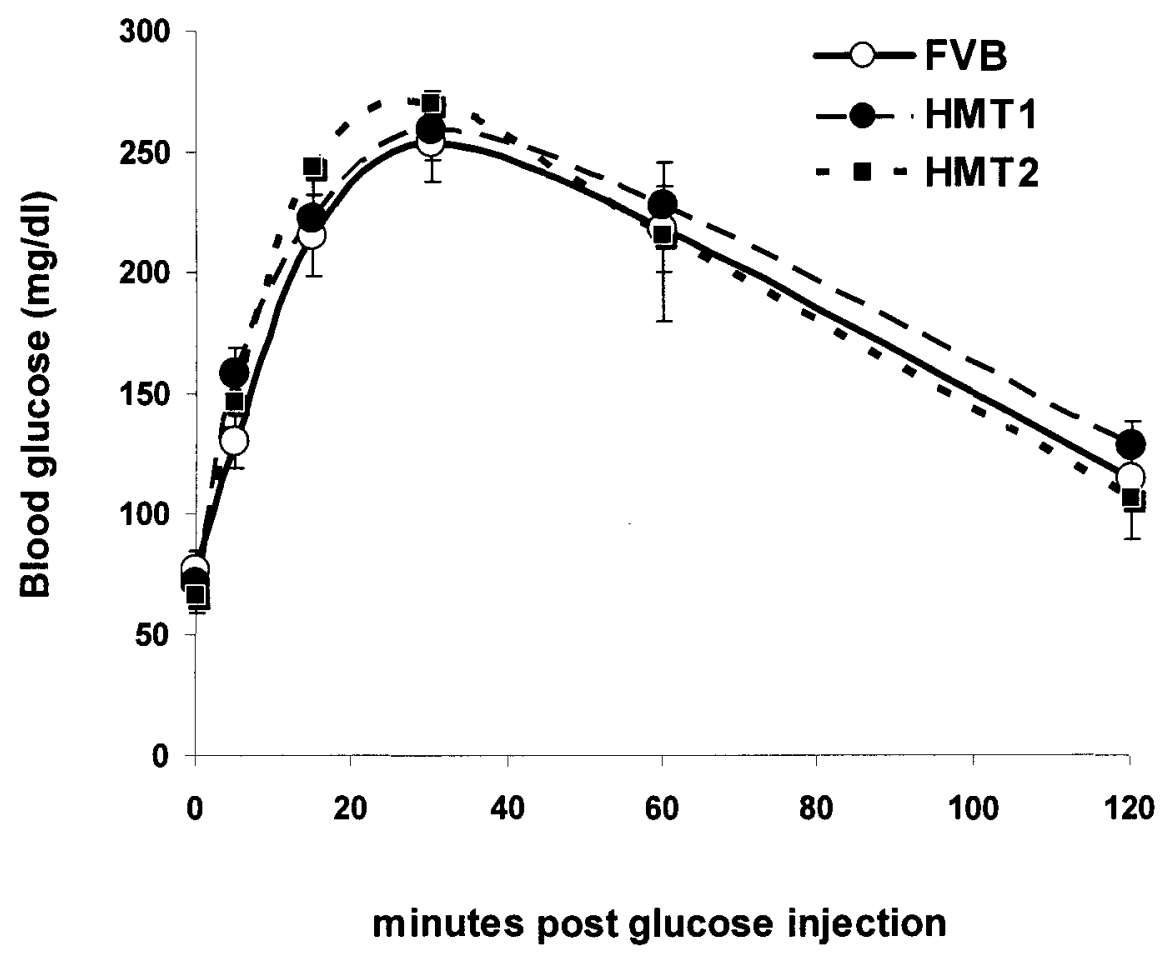

Figure 2-4. Glucose tolerance test in MT transgenic and control FVB mice. Mice were fasted for 16 hrs and then received an intraperitoneal injection of 1 gram glucose/ $\mathrm{kg}$ bodyweight. Blood glucose levels were measured with a Lifescan glucometer at the indicated time after injection. Each group contained at least seven animals. Vertical bars indicate the standard error of the mean. No significant difference was present among either line of transgenic mice and control mice by two-way ANOVA. 


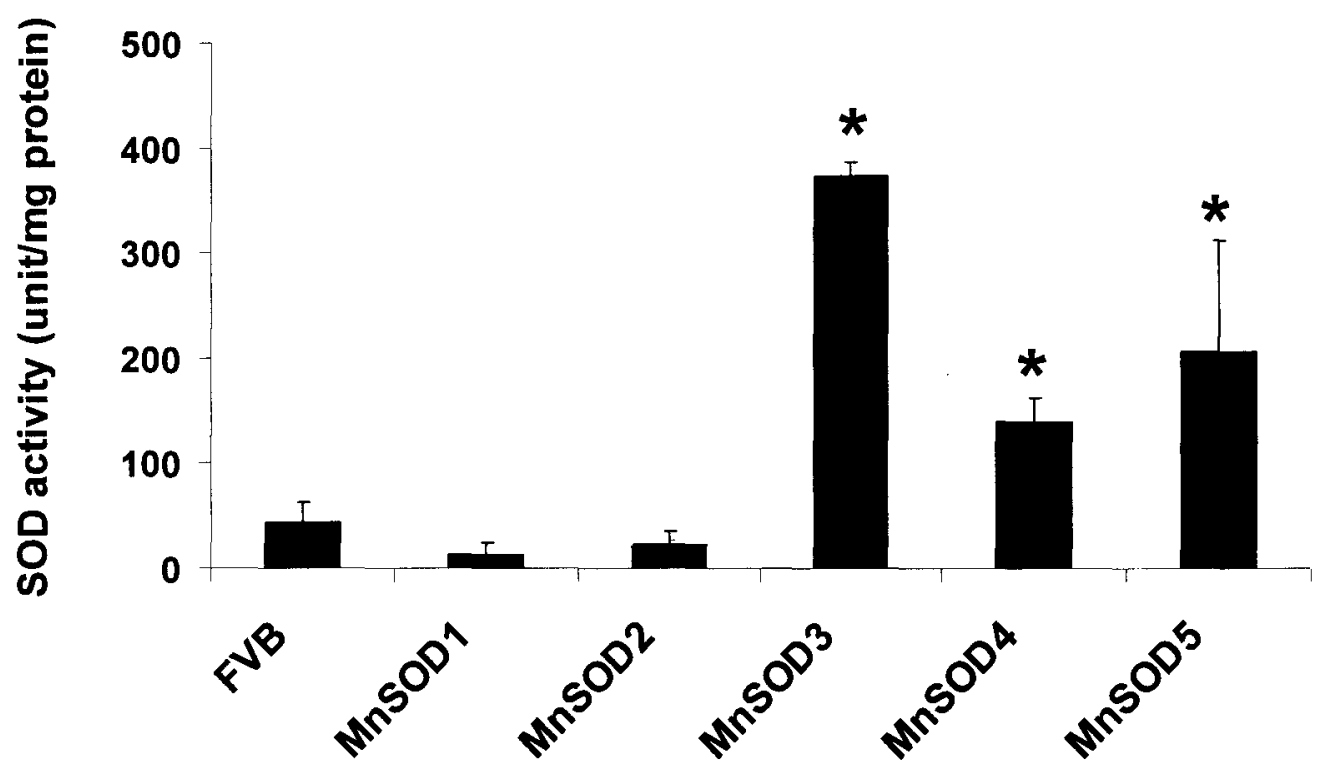

Figure 2-5. SOD activity in isolated islets from five lines of MnSOD transgenic mice and control FVB mice. SOD activity was measured by inhibition of the autooxidation of pyrogallol as described in the Materials and Methods. At least six assays from three islet isolations were performed for each group. Three out of five lines of transgenic mice have elevated pancreatic islet SOD activity. The ${ }^{*}$ indicates different from FVB ( $\mathrm{p}<0.01$ by one way ANOVA). Vertical bars indicate the standard error. 


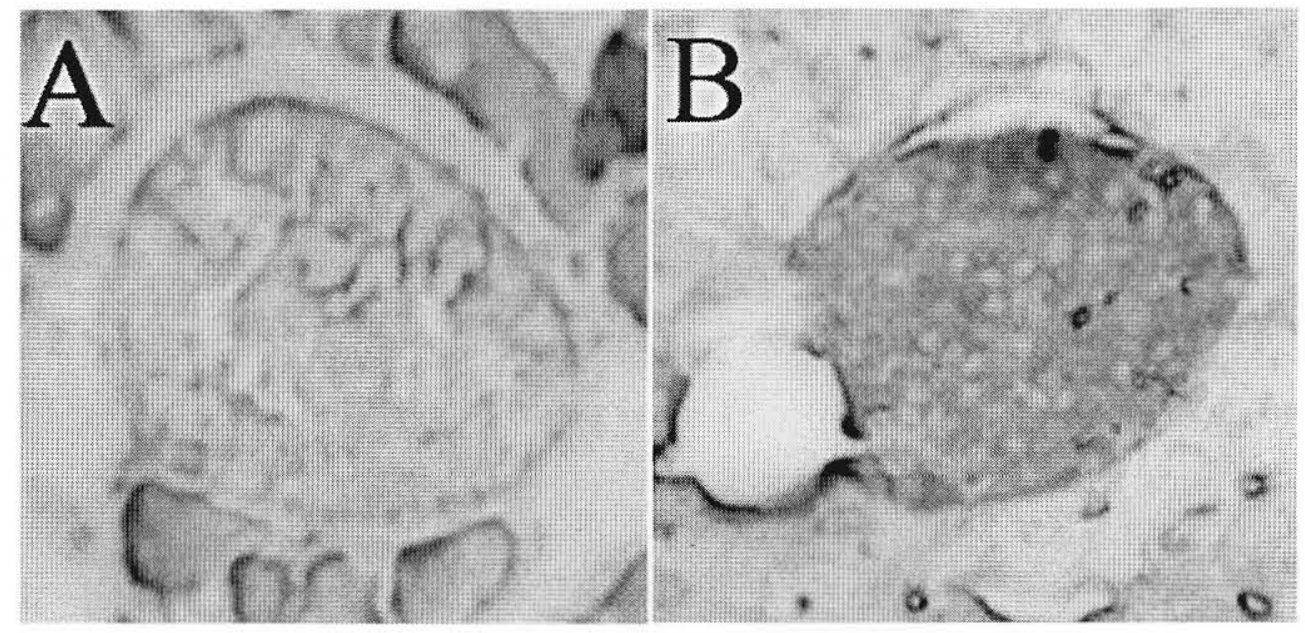

Figure 2-6. Elevated SOD immunostaining in MnSOD transgenic islets compared to FVB control islets. (A) FVB, (B) MnSOD5. Pancreas sections were stained with a sheep anti-human MnSOD primary antibody. Magnification $\times 400$. 


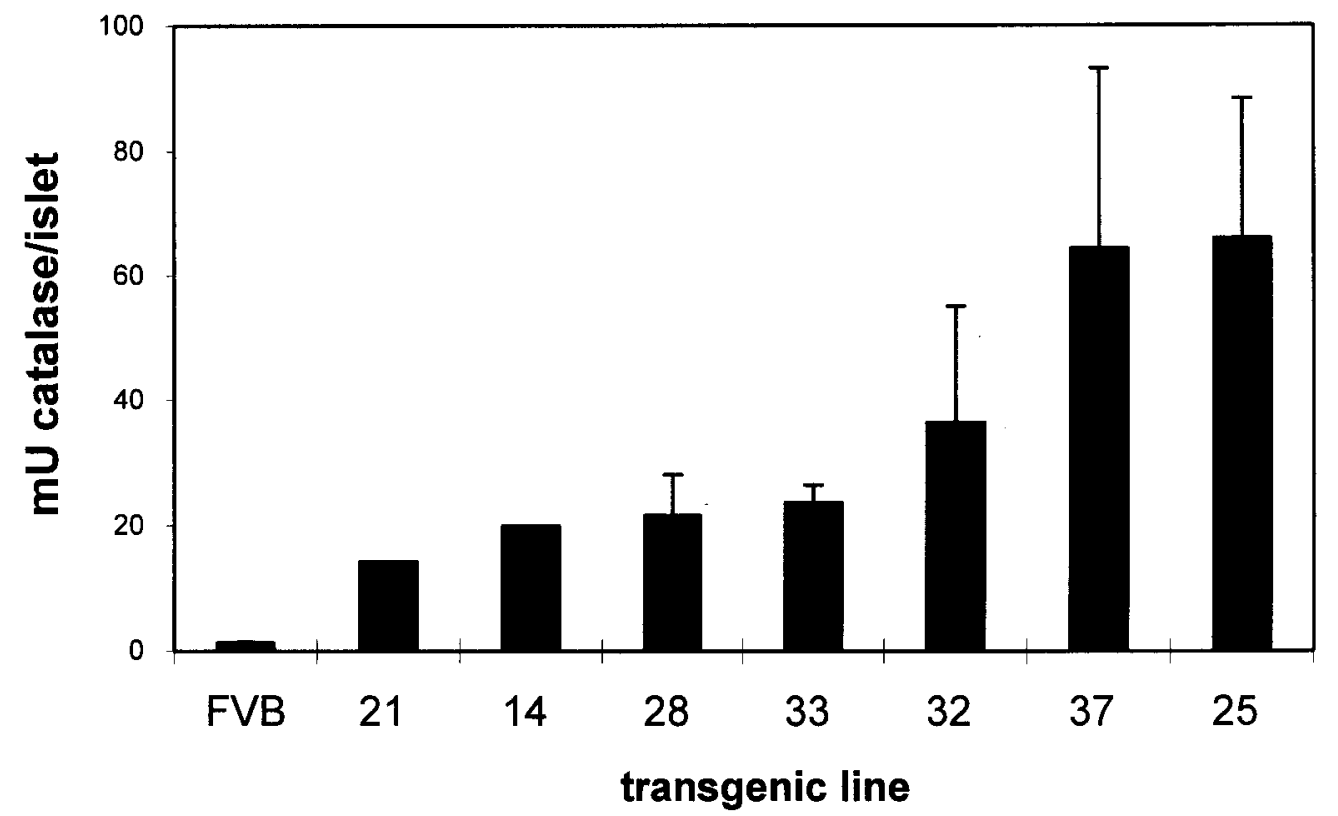

Figure 2-7. Increased catalase activity in isolated islets of catalase transgenic mice. Activity is expressed as equivalent milliunits of purified bovine catalase per islet. Three or more assays were performed on at least 100 islets from each transgenic line. Vertical bars indicate the standard error. Graph was adapted from the work of Dr. Bo Xu, et al. (Xu et al. 1999). 

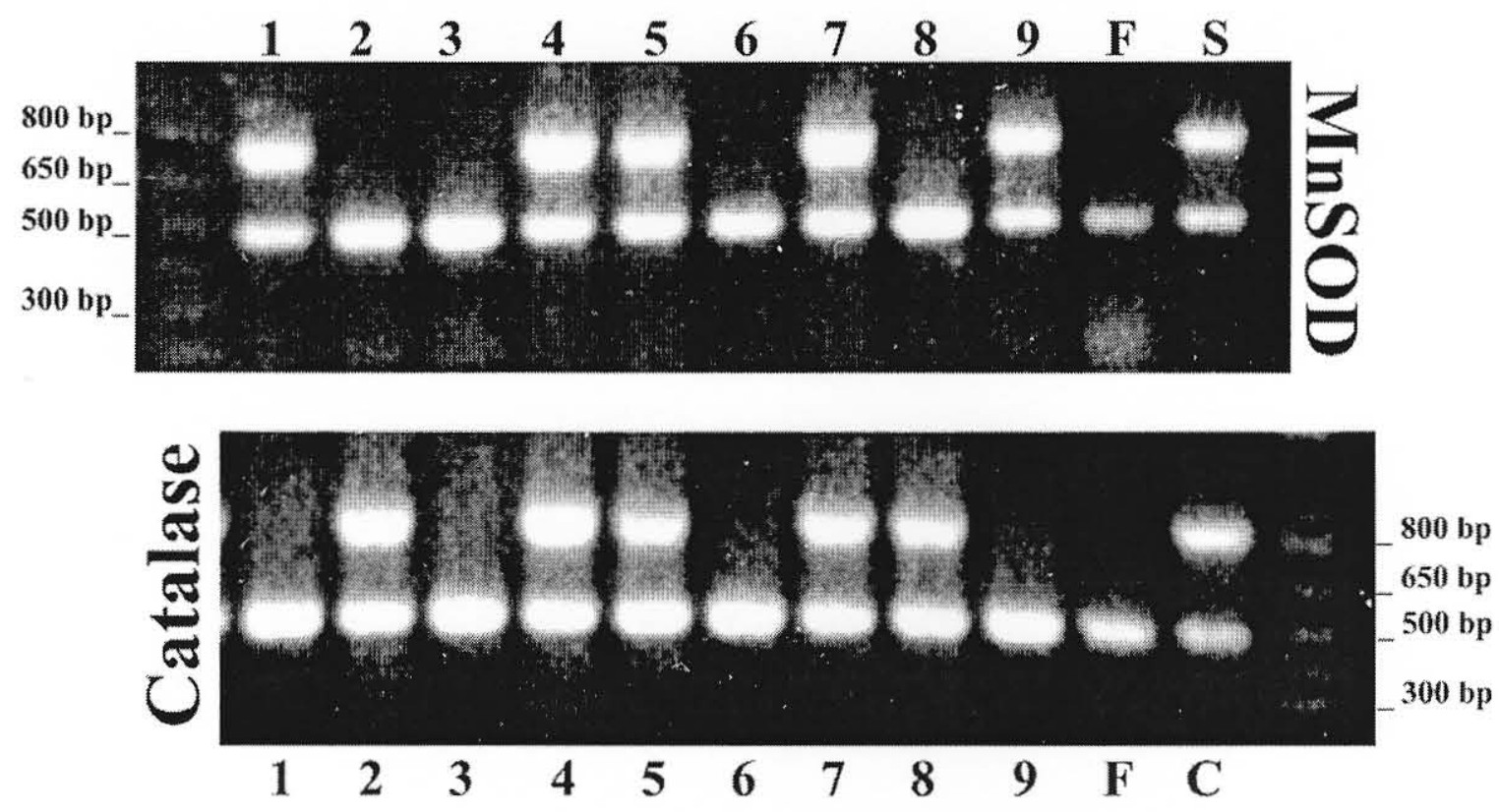

Figure 2-8. PCR identification of MnSOD and catalase transgenes in offspring from cross-breeding of MnSOD (line 3) and catalase (line 25) transgenic mice. This is a typical gel showing PCR products of MnSOD and catalase transgenes. Each lane represents an individual mouse. Lane 1 to Lane 9 are DNA samples from the offspring; (F) negative control of FVB mouse DNA; (S) positive control for MnSOD transgene, (C) positive control of catalase transgene. MnSOD transgene gives a product about $800 \mathrm{bp}$; Catalase transgene gives a product about $850 \mathrm{bp}$; the band with a size about $490 \mathrm{bp}$ is a product of heme oxygenase gene which is expressed in every type of our mice. Mice 1 and 9 carried only MnSOD transgene, mice 2 and 8 carried catalase transgene, and only mice 4,5 and 7 carried both transgenes, whereas mice 3 and 6 did not contain any transgene. 

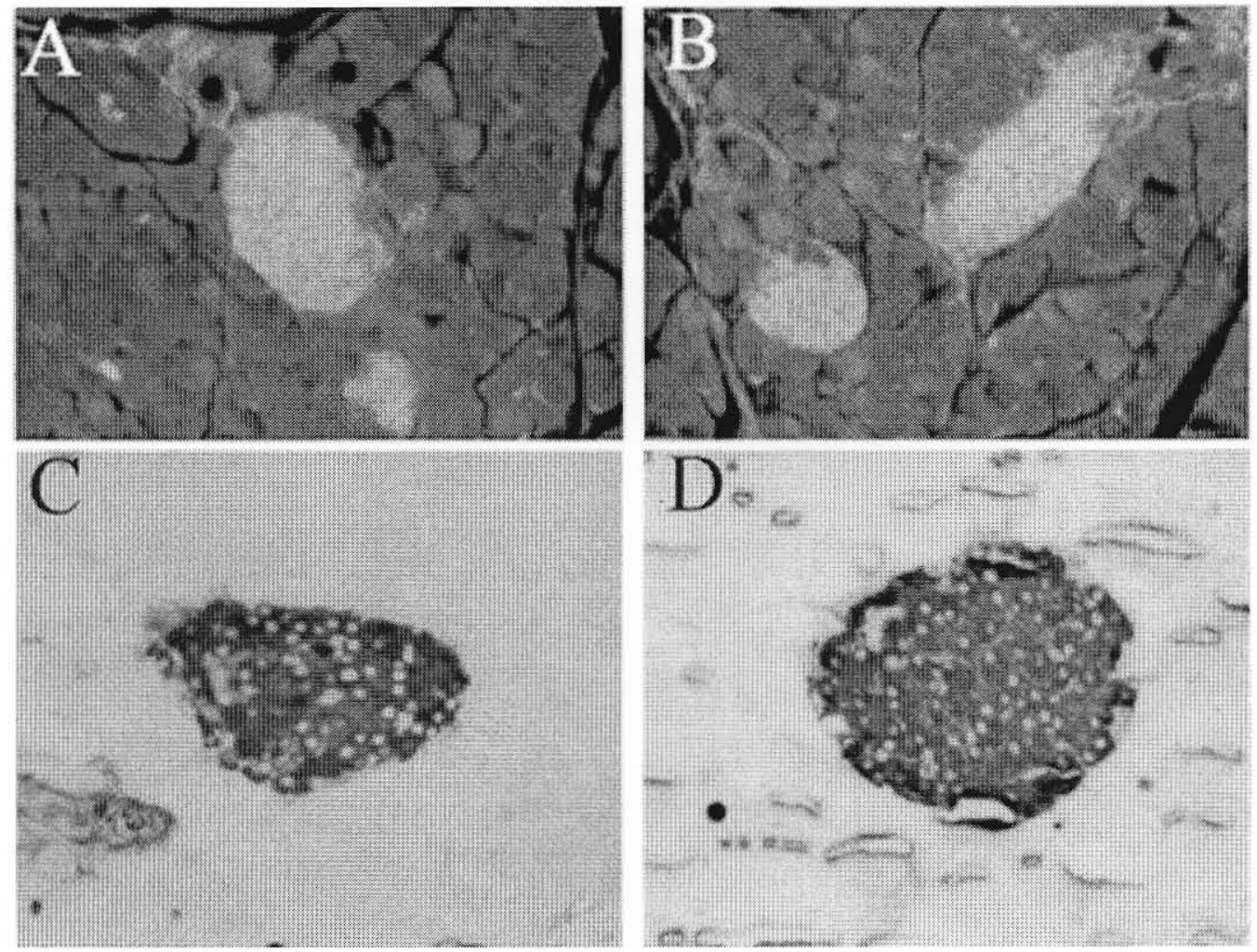

Figure 2-9. Normal insulin immunostaining and islet morphology in catalase and MnSOD transgenic islets. $\mathrm{A}$ and $\mathrm{C}$ are FVB control islets. $\mathrm{B}$ shows islets from catalase transgenic mice. D shows MnSOD transgenic islets. Pancreas sections were stained with a rabbit anti-guinea pig insulin primary antibody, and colored by fluorescein (A and B) or AEC (C and D). Magnification $\times 200$. 


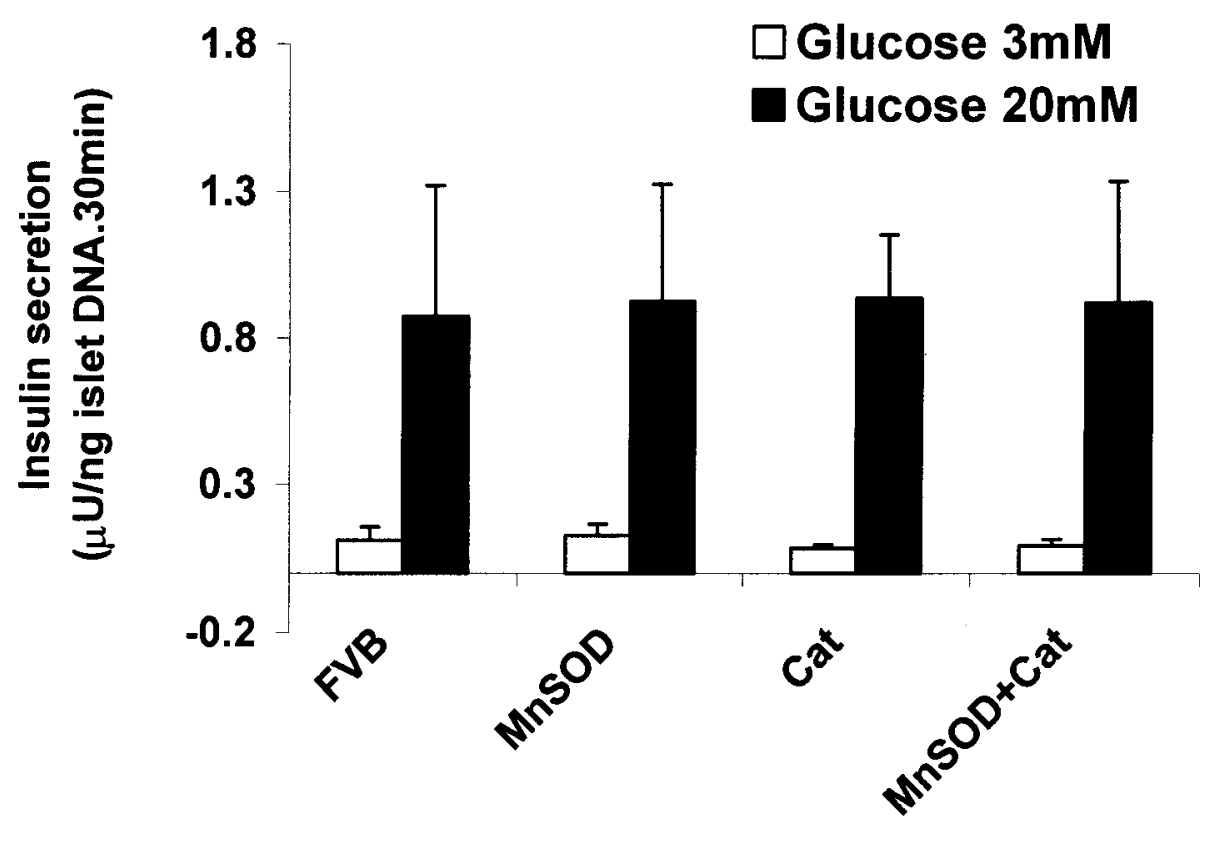

Figure 2-10. Basal (3mM glucose) and $20 \mathrm{mM}$ glucose stimulated insulin secretion in MnSOD, catalase (Cat), MnSOD plus catalase (MnSOD+Cat) transgenic islets and control FVB islets. Insulin secretion assay was performed on 6 to 10 islets in a 96-well plate as described in Materials and Methods. Four independent measurements were carried out per group. Vertical bars indicate the standard error. No statistical difference was found among four groups by two-way ANOVA, indicating that transgenic islets and FVB control islets had similar insulin secretion responses to glucose stimulation. 


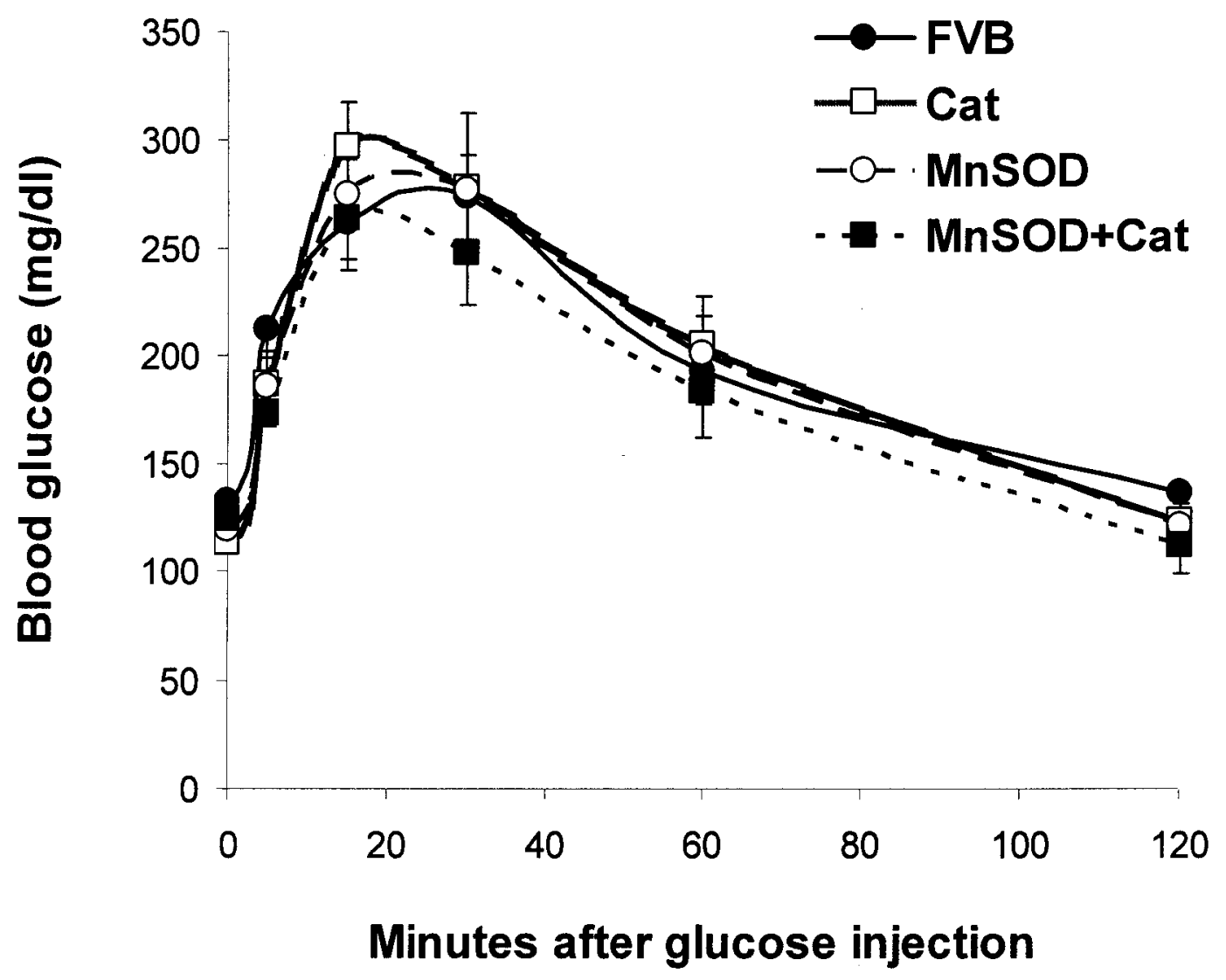

Figure 2-11. Glucose tolerance test in MnSOD, catalase (CAT), MnSOD plus catalase (MnSOD+Cat) transgenic and control FVB mice. Mice were fasted for $16 \mathrm{hrs}$ and then received an intraperitoneal injection of 1 gram glucose/kg bodyweight. Blood glucose levels were measured with a Lifescan glucometer at the indicated time points after injection. Each group contained at least six animals. Vertical bars indicate the standard error. Neither MnSOD/catalase transgene alone nor combination of these two transgenes changed glucose tolerance responses ( $P>0.05$ by two-way ANOVA). 


\section{CHAPTER III}

\section{THE PROTECTIVE EFFECT OF ANTIOXIDANT TRANSGENES AGAINST ROS INDUCED PANCREATIC BETA CELL DESTRUCTION AND STREPTOZOTOCIN INDUCED DIABETES}

\section{Introduction}

In both Type 1 and Type 2 diabetes excessive ROS are thought to be produced in pancreatic beta cells by multiple processes regulated by a variety of factors (Cnop et al. 2001; Ihara et al. 1999; Kessler et al. 1996; Rabinovitch et al. 1992; Rabinovitch 1998). Compared to other cell types, pancreatic beta cells are exceptionally susceptible to ROS induced damage. A striking example for this vulnerability of beta cells is the severe damage done by streptozotocin (STZ). This natural toxin has several destructive actions, including DNA methylation (Murata et al. 1999), protein modification (Liu et al. 2000b), and ROS generation (Ohkuwa et al. 1995). STZ enters the cytoplasm via GLUT2 (Schnedl et al. 1994), which is the ß-cell's glucose transporter. The presence of this transporter may account for part of the specific vulnerability of the B-cell. However, GLUT2 is also present in the liver and kidney, tissues that are relatively resistant to STZ damage. The much greater sensitivity of the ß-cell is probably due to its very low level of antioxidant enzyme expression and activity, particularly hydrogen peroxide-inactivating enzymes catalase and glutathione peroxidase, which are only $2 \%$ as active as they are in liver (Grankvist et al. 1981b; Lenzen et al. 1996; Tiedge et al. 1997). In addition, the adaptive properties of antioxidant enzyme gene expression are very limited in insulin-producing cells (Tiedge et al. 1997). Therefore, increasing pancreatic beta cell antioxidant expression is an attractive strategy to strengthen beta cell resistance to oxidative damage, thereby protecting from diabetic destruction.

A variety of studies have been carried out in vitro using insulin secreting cell lines to test whether elevated antioxidant enzymes could confer protection against ROS damage. Tiedge and his colleagues 
(Lortz et al. 2000; Tiedge et al. 1998; Tiedge et al. 1999) bioengineered RINm5F cells with high expression of glutathione peroxidase, catalase and $\mathrm{Cu} / \mathrm{Zn}$ SOD. Elevated expression of those antioxidants greatly improved cellular resistance of RINm5F cells to oxygen free radicals and NO insults. Moreover those cells are very resistant to cytokine-mediated toxicity. This is also true in other lines of insulin secreting cells. In INS-1 cells the resistance to interleukin-1 $\beta$ is remarkably elevated due to a stable overexpression of MnSOD (Hohmeier et al. 1998), and the resistance to oxidative stress is greatly enhanced through an adenoviral-mediated glutathione peroxidase overexpression (Moriscot et al. 2003). In some other studies increased antioxidant expression in isolated human, rat and porcine islets (Bottino et al. 2002; Benhamou et al. 1998) by adenoviral gene transfer also provided protection from oxidative damage. However, extensive investigations have not been carried out in vivo. Therefore in this project we developed beta cell protected animals instead of beta cell protected cell lines with beta cell specific overexpression of three types of antioxidants: MT, catalase and MnSOD. In this part of the study these animals were evaluated for their resistance to ROS damage and STZ induced diabetes. 


\section{Materials and Methods}

Chemicals: 5- (6)-chloromethyl-2', 7'-dichlorodihydrofluorescein diacetate (CM- $\left.\mathrm{H}_{2} \mathrm{DCFDA}\right)$, picogreen was purchased from Molecular Probes (Eugene, OR). Alarma Blue was bought from Biosource International (Camarillo, CA). $\mathrm{H}_{2} \mathrm{O}_{2}$, hypoxanthine, xanthine oxidase, 3-morpholinosydnonimine (SIN-1), S-nitro-N-acetyl-penicillamine (SNAP), and streptozotocin were bought from Sigma (St. Louis, MO). Polyclonal anti-rabbit nitrotyrosine antibody was supplied by Upstate biotechnology (Charlottesville, VA). Other non-indicated chemicals were standard reagents from Sigma, Fisher Scientific, or Amersham Biosciences.

Dispersion of islets into single cells. Overnight cultured islets were dispersed by trypsin digestion with a modified procedure as described by Appels et al. (Appels et al. 1989). Briefly, 100 to 200 islets were hand picked into $1.5 \mathrm{ml}$ microtube and rinsed with HBSS solution twice. After removal of most medium, $500 \mu \mathrm{l}$ digestion buffer (HBSS solution, $\mathrm{Ca}^{2+}$ and $\mathrm{Mg}^{2+}$-free, no phenol red but containing $0.0075 \%$ trypsin, $3 \mathrm{mM}$ EGTA, $2 \mathrm{mg} / \mathrm{ml}$ Glucose and $20 \mathrm{mg} / \mathrm{ml}$ RIA graded BSA) was added to the islet pellets. Digestion was carried at $37^{\circ} \mathrm{C}$ for 10 minutes. Islets were then mechanically dispersed into single cells by 50 to 80 times of repeated pipetting with a gel loading tip $(0.37 \mu \mathrm{M}$ diameter). After two washes of fresh culture medium (no phenol red), single islet cells were resuspended in $500 \mu$ culture medium without phenol red for next uses.

Measurement of ROS production in dispersed islet cells. A cell membrane-permeable and oxidant sensitive fluorescent dye 5- (6)-chloromethyl-2', 7'-dichlorodihydrofluorescein diacetate (CM$\mathrm{H}_{2}$ DCFDA) was utilized to indirectly measure ROS ( $\mathrm{Li}$ et al. 2003). The dispersed islet cells were loaded with $10 \mu \mathrm{M} \mathrm{CM}-\mathrm{H}_{2} \mathrm{DCFDA}$ for $30 \mathrm{~min}$ followed by three washes of fresh islet culture medium without phenol red. The cells were re-suspended in culture medium without phenol red and cell numbers were counted. These dispersed islet cells loaded with $\mathrm{CM}-\mathrm{H}_{2}$ DCFDA were distributed into a 96-well plate at concentration of 40,000 cells per well in $200 \mu \mathrm{l}$ islet culture medium without phenol red. The exogenous sources of ROS: $\mathrm{H}_{2} \mathrm{O}_{2}$, SIN-1 or hypoxanthine/xanthine oxidase, were quickly added to the wells. Right 
after addition of ROS, the increase of fluorescence intensity in each well was measured continuously for 4 to 6 hours at room temperature on a fluorescent microplate reader (Tecan, Durham, NC) at an excitation wavelength of $485 \mathrm{~nm}$ and an emission wavelength of $530 \mathrm{~nm}$. The data were expressed as increased fluorescent intensity per well.

Cell viability assay by Alamar Blue. The Alamar Blue assay, which incorporates a redox indicator that changes color and fluorescence in response to cell metabolic activity, is a commonly used method to assess cell viability and/or proliferation of mammalian cells (Gonzalez \& Tarloff 2001) and microorganisms (Byth et al. 2001). In our studies, 15 overnight-cultured FVB control islets or transgenic islets were hand picked into $200 \mu$ f fresh culture medium (no phenol red) containing 1:20 diluted Alamar Blue in a 96-well plate. Islets were cultured for $4 \mathrm{hr}$ and the Alamar Blue fluorescence was measured on a fluorescent microplate reader (Tecan, Durham, NC) at the excitation wavelength of $535 \mathrm{~nm}$ and the emission wavelength of $595 \mathrm{~nm}$. This measurement provided an absorbance value indicating the pretreatment metabolic activity and was used to normalize the post-treatment metabolic activity. After three washes with fresh culture medium, islets were cultured in $200 \mu$ culture medium containing various agents, including $\mathrm{H}_{2} \mathrm{O}_{2}$ and STZ, at various concentrations for varying time. In the end of treatment, fifty $\mu \mathrm{l}$ culture medium was replaced with $50 \mu \mathrm{l}$ fresh culture medium containing 1:5 diluted Alamar Blue, for a final dilution of 1:20. The color was developed for another $4 \mathrm{hr}$ and the fluorescence was measured again. A fluorescent value from the "blank well", which contained no islets but exactly followed the above procedure with highest level of ROS treatment, was deducted from all other fluorescent values. Islet cell viability was calculated as the ratio of fluorescence after treatment to the fluorescence before treatment. The untreated islet cell viability was arbitrarily set as $100 \%$.

Nitric oxide in vitro studies. Isolated FVB and HMT-1 islets were exposed to different concentrations of a nitric oxide donor, SNAP for 24 hours. Apoptotic and necrotic DNA were detected with an anti-histone biotin/anti-DNA POD ELISA ${ }^{\text {plus }}$ kit (Roche, Indianapolis, IN) based on the manufacturer's instructions. Briefly, 40 to 50 islets were cultured for $24 \mathrm{hrs}$ in $500 \mu \mathrm{l}$ fresh culture medium in a $1.5 \mathrm{ml}$ 
microtube with or without SNAP treatment. After treatment the microtube was centrifuged at $200 \times \mathrm{g}$ for 10 minutes at $4{ }^{\circ} \mathrm{C}$. The supernatant was removed as the necrosis DNA sample. The pellet was lysed with 100 $\mu 1$ lysis buffer for 30 minutes at room temperature. The microtube was centrifuged again at $200 \times \mathrm{g}$ for 10 minutes at $4^{\circ} \mathrm{C}$. The supernatant was removed as apoptotic DNA sample. To quantify the necrotic and apoptotic DNA, both DNA samples were added to the streptavidin-coated microplate contained in this antihistone biotin/anti-DNA POD ELISA ${ }^{\text {plus }}$ kit. All values were normalized to islet total DNA measured by picogreen DNA quantification (Molecular Probes, Eugene, OR).

In vitro STZ treatment. Overnight incubated islets were handpicked into $2 \mathrm{ml}$ 'for islet DNA damage and $\mathrm{NAD}^{+}$measurements in 24-well tissue culture plate) or $200 \mu \mathrm{l}$ (for islet cell viability and insulin content assays in 96-well tissue culture plate) islet culture medium. Within 1 min before use, STZ was dissolved in $4^{\circ} \mathrm{C} 0.1 \mathrm{M}$ sodium citrate $(\mathrm{pH} 4.5)$. Ten microliters of this solution were added to the islets to obtain the required $\mathrm{STZ}$ concentrations. Islets were incubated at $37^{\circ} \mathrm{C}$ in humidified air and $5 \% \mathrm{CO}_{2}$ for a period of time as indicated in the Results. After treatment, the islets were retrieved for assessment of cell viability assayed by Alamar Blue, preserved islet insulin content measured by an anti-insulin antibody coated tube RIA kit (Diagnostic Products, Los Angeles, CA) in MnSOD and catalase transgenic islets, and measurement of DNA fragmentation and NAD ${ }^{+}$level in HMT-1 transgenic islets.

Analysis of STZ fragmentation of islet DNA. STZ-treated and untreated islets were washed with HBSS and then digested with $50 \mu \mathrm{l} 200 \mu \mathrm{g} / \mathrm{ml}$ proteinase $\mathrm{K}$ in $100 \mathrm{mmol} / \mathrm{l} \mathrm{Tris-} \mathrm{HCl}, \mathrm{pH} 8.5,5 \mathrm{mmol} / 1$ EDTA, and $0.2 \%$ SDS at $55^{\circ} \mathrm{C}$ for $2 \mathrm{~h}$. The islet DNA was then purified with the Prep-A-Gene DNA purification kit from Bio-Rad. After quantification of DNA concentration using the picogreen kit from Molecular Probes, equal amounts of sample DNA were loaded on a $0.3 \%$ SeaKem Gold agarose (FMC Bioproducts) gel. The gel was run at 21 Volts in $0.04 \mathrm{~mol} / \mathrm{l}$ Tris-acetate and $0.001 \mathrm{~mol} / \mathrm{l}$ EDTA buffer for 6 $\mathrm{h}$ and stained with $0.5 \mu \mathrm{g} / \mathrm{ml}$ ethidium bromide.

Measurement of STZ-induced depletion of $\mathrm{NAD}^{+}$. STZ-treated and untreated islets were washed in HBSS and disrupted in $250 \mu \mathrm{l}$ precooled $0.1 \mathrm{~mol} / \mathrm{l}$ bicine ( $\mathrm{pH} 7.8$ ) by freeze/thaw, followed by sonication. 
The sample was centrifuged at $16,000 \times g$ for $10 \mathrm{~min}$, and $100 \mu \mathrm{l}$ of the supernatant was applied for $\mathrm{NAD}^{+}$ determination by an alcohol dehydrogenase cycling method (Shah et al. 1995). Briefly, $100 \mu$ of supernatant was reacted with $100 \mu \mathrm{l}$ freshly prepared reagent mix (to obtain final concentration of $0.1 \mathrm{~mol} / 1$ bicine [pH 7.8], $0.5 \mathrm{~mol} / \mathrm{l}$ ethanol, $4.17 \mathrm{mmol} / 1$ EDTA-Na $4,0.83 \mathrm{mg} / \mathrm{ml} \mathrm{BSA}, 0.42 \mathrm{mmol} / 1$ 3-[4,5dimethylthiazol-2-yl]-diphenlyltetrazolium bromide [MTT], $1.66 \mathrm{mmol} / \mathrm{l}$ phenazine ethosulfate, and 1.25 units $/ \mathrm{ml}$ alcohol dehydrogenase). The reaction was carried out on a 96-well microplate. Color was developed in the dark at room temperature for $45 \mathrm{~min}$, and the absorbance was read at $560 \mathrm{~nm}$. Sample $\mathrm{NAD}^{+}$concentrations were calculated from an $\mathrm{NAD}^{+}$standard curve. Values were expressed per microgram of islet protein in the sonicate, as measured with the Pierce BCA kit.

In vivo STZ treatment. For MT transgene study, a total of 12 HMT-2 mice, 16 HMT-1 mice, and 26 control FVB mice, and for MnSOD/catalase trangene study, a total of 16 control FVB mice, 16 catalase mice, $15 \mathrm{MnSOD}$ mice, and $16 \mathrm{MnSOD}$ plus catalase mice, received a single intraperitoneal injection of freshly dissolved STZ in $0.1 \mathrm{~mol} / \mathrm{l}$ sodium citrate $(\mathrm{pH} 4.5)$ at a dose of $220 \mathrm{mg} / \mathrm{kg}$ body weight. Mice were sex-matched at the age between 10 and 17 weeks. The tail blood glucose levels were monitored daily up for eight days after STZ treatment by a glucometer (OneTouch Ultra, Life Scan, Milpitas, CA). Some mice were sacrificed 30 or 48 hours after STZ administration. The pancreata were removed and sectioned for immunohistochemical staining of nitrotyrosine, or the pancreatic islets were isolated for electron microscopy.

Immunohistochemistry for nitrotyrosine. STZ untreated or treated mouse pancreata were fixed in $10 \%$ formaldehyde in $0.1 \mathrm{~mol} / \mathrm{L}$ phosphate buffer $(\mathrm{pH} 7.2$ ), dehydrated in an ascending graded series of ethanol, and subsequently infiltrated with paraffin. Serial section were cut at $5 \mu \mathrm{m}$, mounted on polylysinecoated slides, and then deparaffinized in xylenes and a descending graded series of ethanol. To stain nitrotyrosine, slides were treated with target retrieval solution (Dako corporation, Carpinteria, CA), followed by M.O.M mouse Ig blocking reagent (Vector Laboratories, Burlingame, CA). Polyclonal antirabbit nitrotyrosine antibody (Upstate biotechnology, Charlottesville, VA) was added to the slides at a dilution oh 1:800 and incubated overnight at $4{ }^{\circ} \mathrm{C}$. After 3 washes in phosphate-buffered saline, slides were 
incubated with biotinylated anti-mouse IgG reagent, followed by $\mathrm{ABC}$ reagent and developed with $\mathrm{DAB}$ as chromagen. Slides without primary antibody treatment were used as negative control. The positive nitrotyrosine signal was obtained in the slides that were pre-treated with commercial peroxynitrite (Sigma).

Electron microscopy. Isolated pancreatic islets were centrifuged to a soft pellet and prepared for transmission electron microscopy by techniques previously described (Carlson et al. 1997). In brief, fixation was carried out for $2-4 \mathrm{~h}$ in cold $\left(4^{\circ} \mathrm{C}\right)$ Karnovsky's (Karnovsky 1965) fixative (pH 7.4). The pellets were then rinsed in $0.2 \mathrm{~mol} / \mathrm{l}$ sodium cacodylate buffer, postfixed in $1 \% \mathrm{OsO} 4$ at $4^{\circ} \mathrm{C}(90 \mathrm{~min})$, and rinsed with distilled water. Dehydration was carried out in graded ethanols and propylene oxide. Pellets were embedded in Epon/Araldite and cured for $48 \mathrm{~h}$ at $55^{\circ} \mathrm{C}$. Epoxy blocks were thin-sectioned (silver-gray interference color) with a Diatome diamond knife. Sections were mounted on 200- $\mu \mathrm{m}$ mesh naked copper grids and stained with lead citrate and uranyl acetate ( $4 \%$ in absolute ethanol). Sections were observed and photographed in a Hitachi 7500 transmission electron microscope.

Data analysis. Data are presented as the mean \pm standard error. Statistical analysis was performed by one-way or two-way ANOVA and Dunnet's post hoc (2-tailed) test. Computations were accomplished using statistical programs from SPSS (version 10.0) and Sigmastat (version 2.03). 


\section{$\underline{\text { Results }}$}

Enhanced ROS scavenging capacity in transgenic beta cells. To determine whether the antioxidant transgenes enhance pancreatic beta cell ROS scavenging ability, the dispersed single islet cells from control FVB mice and transgenic mice were exposed to different concentrations of superoxide radical produced by hypoxanthine and xanthine oxidase (Figure 3-1), peroxynitrite radical generated from SIN-1 (Figure 3-2) and $\mathrm{H}_{2} \mathrm{O}_{2}$ (Figure 3-3). Beta cell ROS production was measured with $\mathrm{CM}-\mathrm{H}_{2}$ DCFDA that had been preloaded into the islet cells prior to ROS addition. ROS oxidized CM- $\mathrm{H}_{2}$ DCFDA, causes an increase of fluorescence intensity. Thus cellular fluorescence provides an indication of intracellular ROS levels. Our data showed that the catalase transgene made pancreatic beta cells more effective in scavenging all three ROS sources. MnSOD transgenic islet cells disposed of superoxide radical and peroxynitrite more efficiently than control FVB islet cells. However, the MnSOD transgene promoted $\mathrm{H}_{2} \mathrm{O}_{2}$ induced CM$\mathrm{H}_{2}$ DCFDA oxidation, indicating the level of beta cell ROS in the presence $\mathrm{H}_{2} \mathrm{O}_{2}$ of was increased rather than reduced by MnSOD expression. Actually, even under basal conditions, ROS levels in MnSOD transgenic islet cells already tended to be higher than in control FVB islet cells (Figure 3-1 A). The combination of MnSOD and catalase trangenes enabled beta cells to more effectively scavenge all three ROS sources. The islet cells containing both transgenes were especially potent in their ability to detoxify SIN-1 generated peroxynitrite radical compared to the islet cells containing either transgene alone (Figure 3-2). This suggests an additive or synergistic action of MnSOD and catalase in scavenging of peroxynitrite radical.

Since the MnSOD transgene enhanced beta cell ROS production after $\mathrm{H}_{2} \mathrm{O}_{2}$ exposure, it should also be true that MnSOD transgenic islets would have a higher sensitivity to $\mathrm{H}_{2} \mathrm{O}_{2}$ toxicity. Therefore we performed a cell viability assay by measuring islet metabolism with Alamar Blue. As shown in Figure 3-4, catalase alone or MnSOD plus catalase significantly decreased $\mathrm{H}_{2} \mathrm{O}_{2}$ induced islet cell death. However the MnSOD transgene by itself sensitized islet cells to $\mathrm{H}_{2} \mathrm{O}_{2}$ toxicity. From the above data we demonstrated that overexpression of MnSOD and/or catalase enabled pancreatic beta cells to discard most forms of ROS more potently. MnSOD and catalase worked together to dispose of peroxynitrite, but MnSOD itself enhanced $\mathrm{H}_{2} \mathrm{O}_{2}$ toxicity to beta cells. 
MT has been recognized as an ROS scavenger with high competence in scavenging of nitric oxide radicals (Sato \& Bremner 1993), superoxide radicals (Lazo et al. 1998), hydroxyl radicals (Kumari et al. 1998), and peroxynitrite (Cai et al. 2000). In this study a similar CM- $\mathrm{H}_{2}$ DCFDA measurement of ROS was carried out in our laboratory by Xiaoyan Li. The dispersed HMT-1 transgenic and control FVB islet cells were exposed to three ROS sources: superoxide radial from the reaction of hypoxanthine and xanthine oxidase, SIN-1 derived peroxynitrite and $\mathrm{H}_{2} \mathrm{O}_{2}$. As shown in Figure 3-5, beta cell ROS production was dramatically reduced by the MT transgene following exposure to all three ROS sources. Islets were also exposed to nitric oxide by incubation with SNAP, a nitric oxide donor. SNAP did not increase CM$\mathrm{H}_{2}$ DCFDA fluorescence in our assay probably because CM- $\mathrm{H}_{2}$ DCFDA is not sensitive to nitric oxide radical; consequently we assessed MT induced resistance to nitric oxide by observing islet morphological changes and quantitating islet cell death as measured by DNA cleavage. As shown in Figure 3-6, MT transgenic islets were more resistant to SNAP induced morphological damage and cell death than control FVB islets. These data demonstrate that the beta cells containing MT transgene are capable of scavenging all or most forms of ROS.

\section{Protective effect of MnSOD and catalase transgenes against STZ induced beta cell toxicity and}

diabetes. Since MnSOD and/or catalase greatly enhance pancreatic beta cell ROS scavenging capacities, further studies were employed to test whether MnSOD and catalase provided protection against STZ induced beta cell death and diabetes. STZ is a commonly used diabeteogenic agent which produces ROS and specifically destroys pancreatic beta cells (Flodstrom et al. 1999; Ohkuwa et al. 1995). In our study the islet cell viability and preserved islet insulin content were measured in transgenic islets (MnSOD, catalase, or MnSOD plus catalase) and control FVB islets after exposure to STZ for two days. As shown in Figure 37, either MnSOD or catalase transgene tended to reduce STZ induced islet cell death and insulin content loss a little bit. But this protective effect was remarkably amplified when MnSOD and catalase were present together in the beta cell. 
decrease in the strength of the band running above $48 \mathrm{~kb}$ was taken as the primary indicator of STZ-induced DNA damage. MT clearly protected this high-molecular weight band, particularly at the lower doses of STZ. In control islets, the high-molecular weight DNA declined at concentrations between 0.25 and 0.5 mM STZ. In islets overexpressing MT, this degree of degradation did not occur until a concentration of 1.0 mM STZ was reached. DNA protection by MT was found in seven consecutive experiments. These results demonstrated that MT increases the threshold for DNA damage. However, this protection was breached by doubling the dose of STZ. In no instance did we see a DNA ladder characteristic of apoptosis. DNA cleavage by STZ occurs at largely random sites (LeDoux et al. 1986), due to chemical attack rather than regularly spaced sites produced by enzymatic cleavage, as seen in apoptosis.

STZ-induced depletion of cellular $\mathrm{NAD}^{+}$is thought to be a consequence of DNA damage (Masutani et al. 1999). Therefore, we anticipated that MT would protect cellular $\mathrm{NAD}^{+}$content. This was tested in islets exposed to STZ under the same conditions used for DNA damage analysis. As shown in Figure 3-12, MT overexpression right-shifted the dose response curve for depletion of $\mathrm{NAD}^{+}$. Because it is the lack of $\mathrm{NAD}^{+}$ that ultimately causes the downfall of the $\beta$-cell, this result indicates that MT overexpression can prevent $\beta$ cell death and diabetes.

For In vivo analysis, both lines of MT transgenic and control mice received a single injection of STZ. Blood glucose levels were monitored for the next 6 days after STZ administration. The data shown in Figure 3-13 indicated that the onset of diabetes was remarkably delayed by the MT transgene, particularly in HMT-1 mice. In control animals, blood glucose values $>400 \mathrm{mg} / \mathrm{dl}$ usually occurred 2 days after the administration of STZ. A similar degree of diabetes required 3 days in HMT-2 mice and did not occur during the 6-day observation period in HMT-1 mice.

The damage of STZ resulted in morphological changes to islet cells. MT overexpression prevented this destruction. After two days of STZ administration, we isolated islets and made morphological observations in HMT-1 and FVB islets by electron microscopy (Figure 3-14). We found untreated HMT-1 islets appeared normal and were indistinguishable from untreated FVB islets (Figure 3-14 A and B). After 
STZ treatment, islet preparations from FVB mice showed markedly different morphological features (Figure 3-14 C). In these preparations, many cells, putatively identified as beta cells, exhibited several features of necrosis, including vacuolization, moth-eaten mitochondria, dilated cisternae of rough endoplasmic reticulum, and electron-lucent cytoplasm. Beta cells in these preparations showed fewer granules than their untreated counterparts, and many were morphologically disorganized or necrotic. In contrast, islets isolated from STZ-treated HMT-1 transgenic mice showed no evidence of necrosis, and though some beta cells were moderately degranulated, vacuolization and biomembrane discontinuities were not seen (Figure 3-14 D). 


\section{$\underline{\text { Discussion }}$}

In this part of study we explored whether the antioxidant transgenes MnSOD, catalase, MnSOD plus catalase, and MT could effectively scavenge ROS and could protect against ROS induced pancreatic beta cell destruction and STZ-induced diabetes. By measuring CM- $\mathrm{H}_{2}$ DCFDA oxidation, we verified that overexpression of these antioxidants greatly enhanced beta cell ROS scavenging abilities. Cell viability assays, DNA fragmentation assays and insulin content measurements confirmed the protective effect of these antioxidants. The pancreatic beta cells containing these antioxidants proteins, with the exception of MnSOD, were more resistant to the toxicity of $\mathrm{H}_{2} \mathrm{O}_{2}$, nitric oxide and STZ compared with FVB control cells. MnSOD actually increased measured ROS levels and sensitized toxicity to beta cells when exposed to $\mathrm{H}_{2} \mathrm{O}_{2}$. The protective effect against STZ-induced diabetes was also investigated in these antioxidant transgenic mice. The result showed that all of these antioxidant transgenes tended to delay and reduce STZ induced diabetes. The MT transgene significantly suppressed STZ-induced beta cell morphological damage. MnSOD and catalase transgenes worked synergistically to prevent ROS damage as the combination of these two transgenes not only made beta cells more efficient in scavenging peroxynitrite radical and peroxynitrite derived protein nitrotyrosine production, but also made mice more resistant to STZ induced diabetes than either transgene alone.

This synergistic action of MnSOD and catalase comes from their enzymatic actions. The ROS scavenging reactions of these two antioxidants are sequentially linked. MnSOD is located in the mitochondrial which is the main site for production of superoxide radical. MnSOD converts superoxide to $\mathrm{H}_{2} \mathrm{O}_{2}$ and oxygen. $\mathrm{H}_{2} \mathrm{O}_{2}$ can freely diffuse through the mitochondrial membrane into the cytoplasm and peroxisome where catalase is located. $\mathrm{H}_{2} \mathrm{O}_{2}$ is then detoxified by catalase to water. These processes are very important for beta cell survival under oxidative stress since pancreatic beta cells contain very low levels of antioxidant protein expression and activity, particularly catalase and glutathione peroxidase (Lenzen et al. 1996). Simple overexpression of MnSOD will increase $\mathrm{H}_{2} \mathrm{O}_{2}$ production with which the beta cell is poorly equipped to deal. Therefore simultaneous overexpression of both MnSOD and catalase renders the beta cell much more effective in dealing with ROS than either single transgene alone. Not only our results, but results from other studies indicated this benefit of combined antioxidants. In Drosophila 
Melanogaster, overexpression of both SOD and catalase significantly extended its life-time and retarded aging related-protein oxidative damage while neither protein alone was effective (Orr \& Sohal 1994). In the beta cell line RINm5F, combined expression of $\mathrm{Cu} / \mathrm{Zn}$ SOD plus catalase or $\mathrm{Cu} / \mathrm{Zn}$ SOD plus glutathione peroxidase provided more protection against $\mathrm{H}_{2} \mathrm{O}_{2}$, superoxide radical, and NO than single expression of either transgene (Tiedge et al. 1998; Tiedge et al. 1999). In the same RINm5F cells, coexpression of MnSOD and catalase provided an even better protection against $\mathrm{H}_{2} \mathrm{O}_{2}$ than did co-expression of $\mathrm{Cu} / \mathrm{Zn} \mathrm{SOD}$ and catalase, indicating the important role of mitochondria in ROS production (Lortz \& Tiedge 2003). In cultured rat islets, Uchigata et al. (Uchigata et al. 1982) demonstrated that coadministration of SOD and catalase more effectively prevented alloxan induced islet DNA breaks and proinsulin biosynthesis inhibition than did each ROS scavenger alone. All these results suggest that the best protection of pancreatic beta cells against ROS destruction is achieved by a coordinated inactivation of various types of ROS by a combination of antioxidant enzymes rather than any single enzyme in isolation.

Some earlier studies questioned the role of ROS in producing beta cell damage by STZ. In the study of Uchigata et.al. (Uchigata et al. 1982) neither SOD nor catalase was found to prevent STZ induced islet DNA breaks and inhibition of proinsulin synthesis. Also other studies reported that SOD failed to protect against the diabetogenic action of STZ (Gold et al. 1981) Therefore, Uchigata et al. proposed that, unlike alloxan, STZ- induced beta cell DNA damage was mediated by an alkylating activity of STZ itself (Murata et al. 1999) rather than mediated by STZ generated ROS. In our studies we found that MnSOD was protective against STZ, but the effect of MnSOD alone was quite weak. However when combined with catalase, MnSOD was very effective. Therefore that data supports the ROS mechanism of STZ toxicity. Our study with the MT antioxidant also clearly showed protection from STZ induced beta cell DNA fragmentation and beta cell death, further supporting the role of ROS. In fact it has been proven that both NO (Kroncke et al. 1995), hydroxyl radical (Gille et al. 2002), and $\mathrm{H}_{2} \mathrm{O}_{2}$ (Takasu et al. 1991) are generated by STZ and they greatly contribute to beta cell DNA damage. The reason for those studies that failed to observe the protective effect of antioxidants against STZ probably is due to the limitation of their procedure in delivering antioxidants. With their procedure, the amount of antioxidants reaching the target beta cells could not be as high as that achieved by our transgenes. 
Another interesting finding in this study is that MnSOD overexpression appears to increase $\mathrm{H}_{2} \mathrm{O}_{2}$ induced ROS production and sensitizes $\mathrm{H}_{2} \mathrm{O}_{2}$ toxicity to beta cells. This finding is consistent with some other studies performed in beta cell lines where SOD either is shown to be not protective against or sensitizing to the toxicity of some types of ROS. For example, in insulin secreting RINm5F cells overexpression of SOD does not alter the resistance of the cells to $\mathrm{H}_{2} \mathrm{O}_{2}$ but sensitized to the toxicity of menadione and butylalloxan (Tiedge et al. 1998; Tiedge et al. 1999). This increased sensitivity due to SOD overexpression is more widely reported in the field of cancer research (Kim et al. 2001; Li et al. 1999; Zhong et al. 1996). In numerous transformed cancer cell lines, the overexpression of MnSOD results in an increased ROS level under basal cell culture condition ( $\mathrm{Li}$ et al. 1998), and leads to reversion of tumorigenicity in vivo (Church et al. 1993; Kim et al. 2001) and loss of the malignant phenotype in vitro (Li et al. 1998; Zhong et al. 1996). Unfortunately, the mechanism underlying the tumor-suppressing ability of MnSOD has not been defined. A prevailing thought is that an imbalance in the redox state of the cells causes an inhibition of cell growth, though the precise role of excess MnSOD is not known. In theory, scavenging of superoxide anion by MnSOD leads to an increase in $\mathrm{H}_{2} \mathrm{O}_{2}$, and without a concomitant increase in the peroxide-scavenging enzymes, this oxygen intermediate may become toxic. In the mitochondrial microenvironment, excess $\mathrm{H}_{2} \mathrm{O}_{2}$ would be available to react with $\mathrm{Fe}^{2+}$, contained in multiple Fe-S electron transport chain components, leading either to the production of hydroxyl radical via HaberWeiss reaction (Liochev 1996) or of ferry or perferryl species (Harel \& Kanner 1988).

In our studies, the $\mathrm{H}_{2} \mathrm{O}_{2}$ was added in the culture medium. Some ROS produced could enter the mitochondria and form superoxide. Excess MnSOD would convert this to $\mathrm{H}_{2} \mathrm{O}_{2}$ which through the HaberWeiss reaction can form more potent ROS such as hydroxyl radical. Thereby the overall effect on beta cells would be more toxic. However if catalase was also overexpressed in the beta cells, $\mathrm{H}_{2} \mathrm{O}_{2}$ would be decomposed rapidly.

The protective value of MT in the $\beta$-cell was anticipated because MT is an antioxidant protein (Sato \& Bremner 1993), B-cell toxins induce it (Minami et al. 1999; Ohly \& Gleichmann 1995; Ohly et al. 
2000), and MT has demonstrated efficacy against DNA damage in other cell types (Chubatsu \& Meneghini 1993). These facts led several laboratories to examine whether increased levels of MT could produce a more resistant $\beta$-cell. Initial results were very promising. Ohly et al. (Ohly \& Gleichmann 1995)showed that, after STZ treatment in vitro, induction of MT with zinc pretreatment helped preserve islet function and prevented diabetes induced by multiple low doses of STZ treatment in vivo (Ohly et al. 2000). However, zinc has actions other than MT induction (Bray \& Bettger 1990; Vallee \& Falchuk 1993). A particularly relevant example of zinc actions that are independent of MT is shown in the report by Apostolova et al. (Apostolova et al. 1997), who found that, in mice lacking functional MT I and II genes, zinc treatment reduces several actions of STZ, including hypoinsulinemia and elevated blood glucose. Therefore zinc can be protective even without induction of MT. In this study we utilized a far more specific approach than zinc pretreatment to elevate MT in pancreatic beta cells. Therefore the results of our study clearly confirmed the original suggestion that MT can protect B-cells.

In conclusion, for this part of study, we demonstrated that elevated activity of ROS scavengers is beneficial for beta cell survival after exposure to oxidative stress. This result has potential therapeutic values for diabetes therapy. First, overexpression or pretreatment with antioxidant compounds or proteins on cultured islets may produce a donor islet highly resistant to ROS damage for islet transplantation. Several studies have demonstrated that this is a feasible approach to improve the chance of successful islet transplantation. Pretreatment with a SOD mimic compound significantly preserved cultured islet mass (Bottino et al. 2002) and gene transfer of MnSOD by adenovirus remarkably extended islet graft function in diabetic NOD recipient (Bertera et al. 2003). Second, induction of the endogenous antioxidant genes may provide an alternative strategy for producing a more robust $\beta$-cell, without the need to insert foreign genes. Many beta cell antioxidant genes such as MnSOD, thioredoxin and MT, are inducible. Pretreatment with some agents such as glucocorticoids is able to increase antioxidant expression by over 10 -fold in some cell types (Palmiter et al. 1993). This increase is similar to the 4- to 30 -fold increases we obtained with our transgene. Therefore, developing and applying a specific reagent to induce beta cell antioxidants might be a valuable approach for clinic diabetes treatment. 
However, in this part of the study we only focused on ROS scavenging property of these antioxidant transgenes. Neither in vitro exposure to ROS producing compounds nor in vivo production of STZ diabetes closely resembles human Type 1 and Type 2 diabetes. The results from this part only suggest that the pancreatic beta cells in our transgenic mice become resistant to ROS damage. Cytokines may be more important mediators for beta cell death in Type 1 diabetes. Therefore, in the next part of this project we studied the potential role of antioxidant transgenes in cytokine mediated beta cell dysfunction and cell death. 

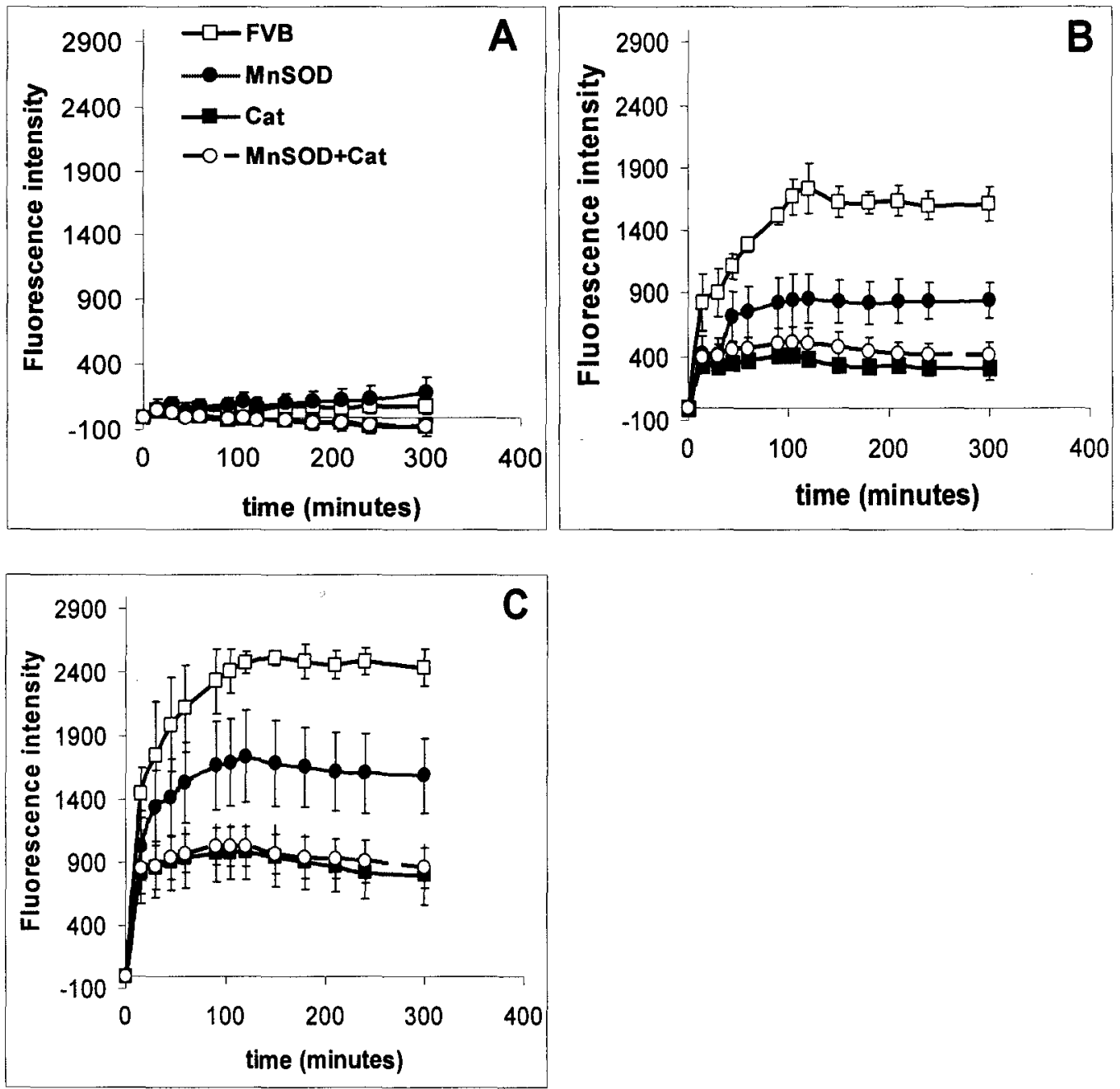

Figure 3-1. Superoxide radical in dispersed MnSOD, catalase (Cat), MnSOD plus catalase (MnSOD+Cat) transgenic and control FVB islet cells. ROS production was measured with oxidant sensitive fluorescent probe $\mathrm{CM}-\mathrm{H}_{2}$ DCFDA as described in Materials and Methods. Cells were treated with vehicle containing $1 \mathrm{mM} \mathrm{NaOH}$ (panel A), or $1 \mathrm{mM}$ hypoxanthine (dissolved in $1 \mathrm{mM} \mathrm{NaOH}$ ) plus $2 \mathrm{mU}$ xanthine oxidase (panel B), or $1 \mathrm{mM}$ hypoxanthine plus $10 \mathrm{mU}$ xanthine oxidase (panel C). Either catalase and MnSOD alone or combination of MnSOD and catalase decreased islet cell superoxide radical generated from the reaction of hypoxanthine and xanthine oxidase. Data were calculated from three or four independent measurements. Vertical bars represent standard error. 

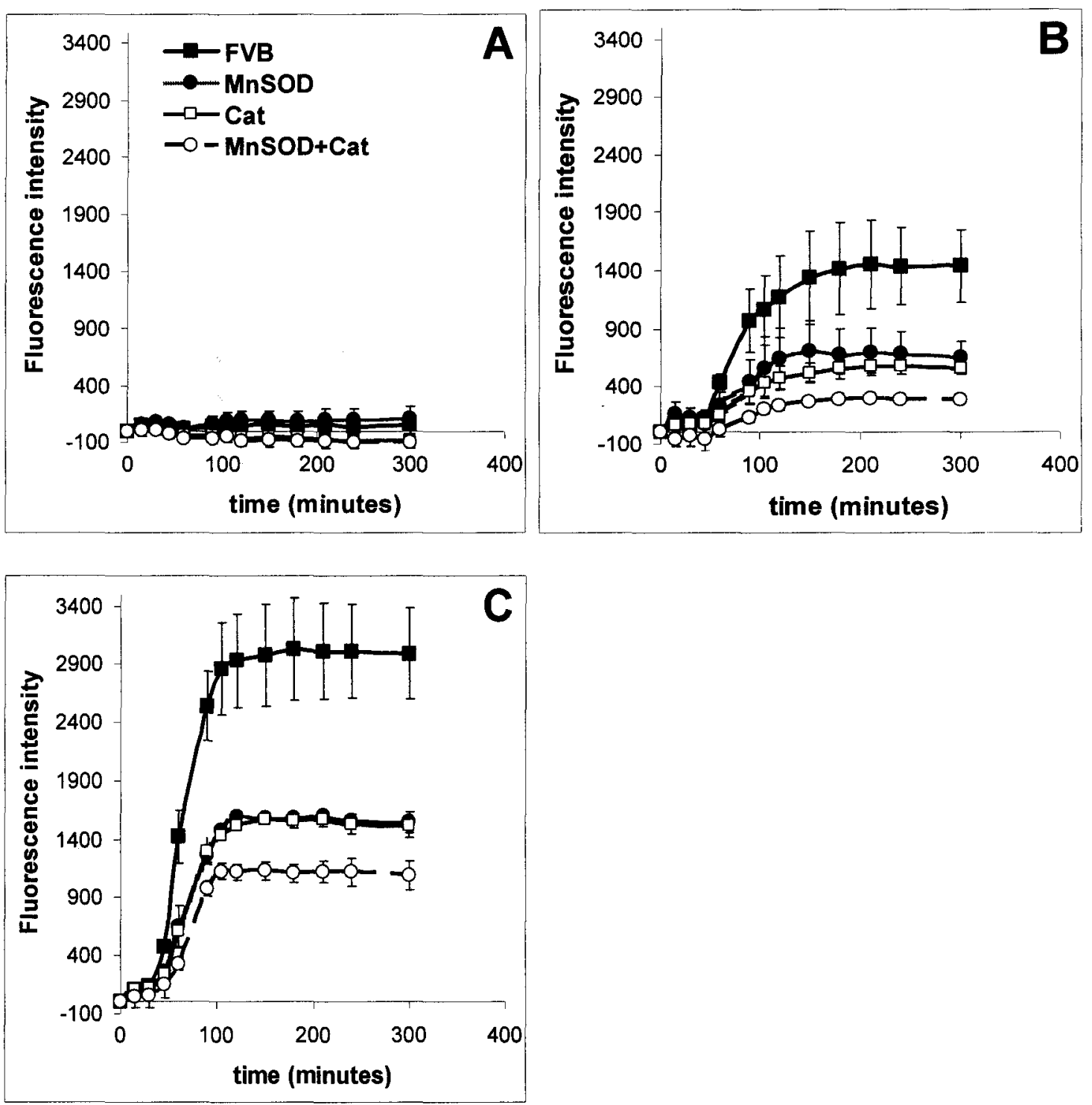

Figure 3-2. ROS production in dispersed MnSOD, catalase (Cat), MnSOD plus catalase (MnSOD+Cat) transgenic and control FVB islet cells after treatment with SIN-1. ROS production was measured with oxidant sensitive fluorescent probe $\mathrm{CM}-\mathrm{H}_{2}$ DCFDA as described in Materials and Methods. Cells were not treated (panel A), or treated with SIN-1 $30 \mu \mathrm{M}$ (panel B) and $100 \mu \mathrm{M}$ (panel C). Either catalase or MnSOD remarkably decreased ROS production in islet cells. MnSOD plus catalase reduced ROS in islet cells more potently than either single transgene, indicating a synergistic action of MnSOD and catalase to dispose of SIN-1 induced ROS production. Data were calculated from three or four independent measurements. Vertical bars represent standard error. 

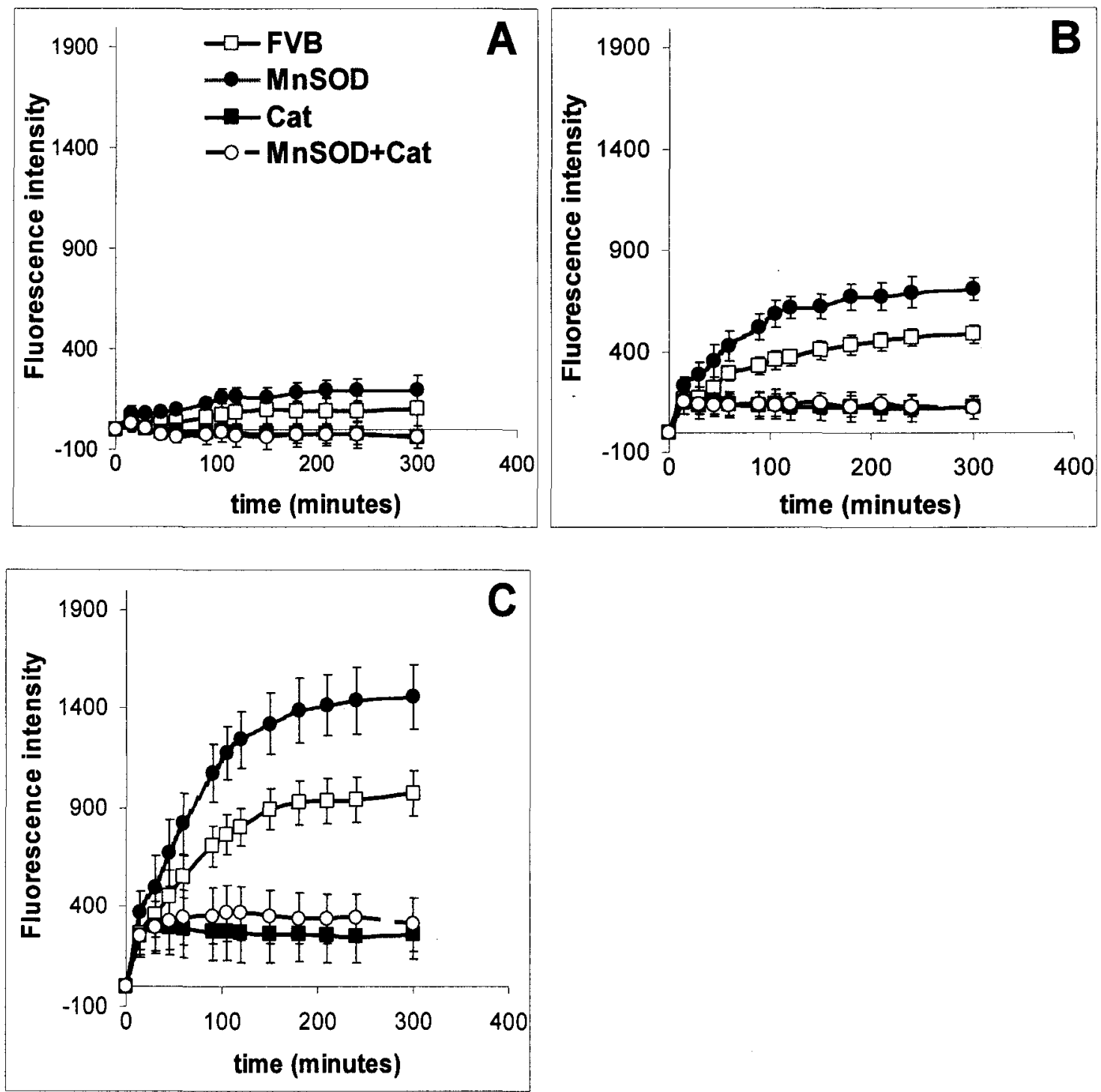

Figure 3-3. ROS production in dispersed MnSOD, catalase (Cat), MnSOD plus catalase (MnSOD+Cat) transgenic and control FVB islet cells after treatment with $\mathrm{H}_{2} \mathrm{O}_{2}$. ROS production was measured with oxidant sensitive fluorescent probe $\mathrm{CM}-\mathrm{H}_{2}$ DCFDA as described in Materials and Methods. Cells were not treated (panel A), or treated with $\mathrm{H}_{2} \mathrm{O}_{2} 20 \mu \mathrm{M}$ (panel B) and $100 \mu \mathrm{M}$ (panel C). Either catalase or MnSOD plus catalase remarkably decreased ROS production in islet cells. However, MnSOD alone increased DCF signal, indicating more ROS were produced in MnSOD transgenic islet cells after treatment with $\mathrm{H}_{2} \mathrm{O}_{2}$. Data were calculated from three or four independent measurements. Vertical bars represent standard error. 


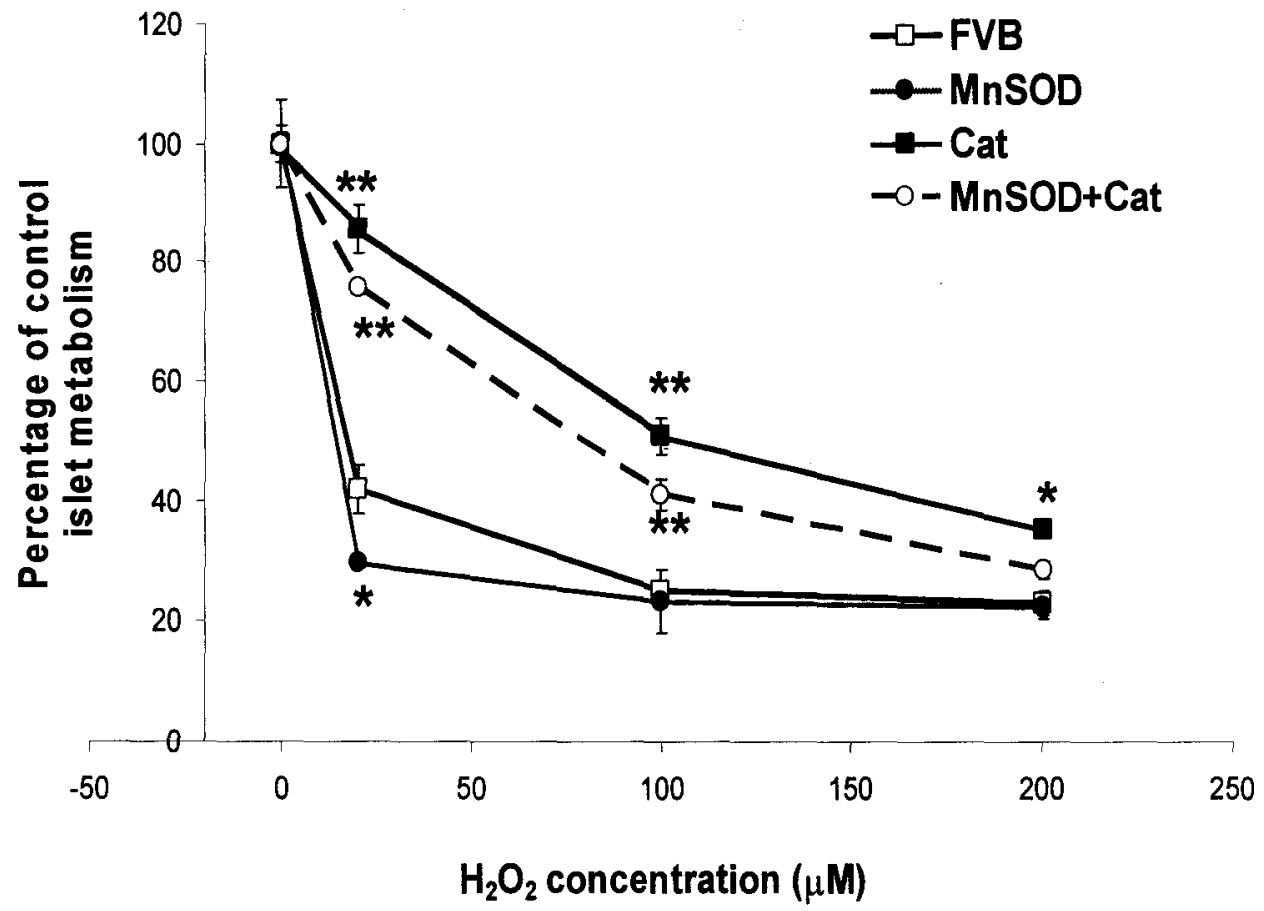

Figure 3-4. Cell viability in MnSOD, catalase (Cat), MnSOD plus catalase (MnSOD+Cat) transgenic islets and control FVB islets after treatment with $\mathrm{H}_{2} \mathrm{O}_{2}$ for 12 hours. Islet cell viability was calculated as percentage of control islet cell metabolism indicated by Alamar Blue absorbance as described in Materials and Methods. Data were mean $\pm \mathrm{SE}$ from four independent measurements. Vertical bars represent standard error. ${ }^{*} \mathrm{P}<0.05$ and ${ }^{* *} \mathrm{P}<0.01$ between the indicated transgenic islets and FVB control islets at the corresponding concentration of $\mathrm{H}_{2} \mathrm{O}_{2}$ by two-way ANOVA. Either catalase alone or MnSOD plus catalase significantly reduced $\mathrm{H}_{2} \mathrm{O}_{2}$ induced islet cell death. But MnSOD alone sensitized $\mathrm{H}_{2} \mathrm{O}_{2}$ toxicity to islet cells at the lowest concentration of $\mathrm{H}_{2} \mathrm{O}_{2}$. 

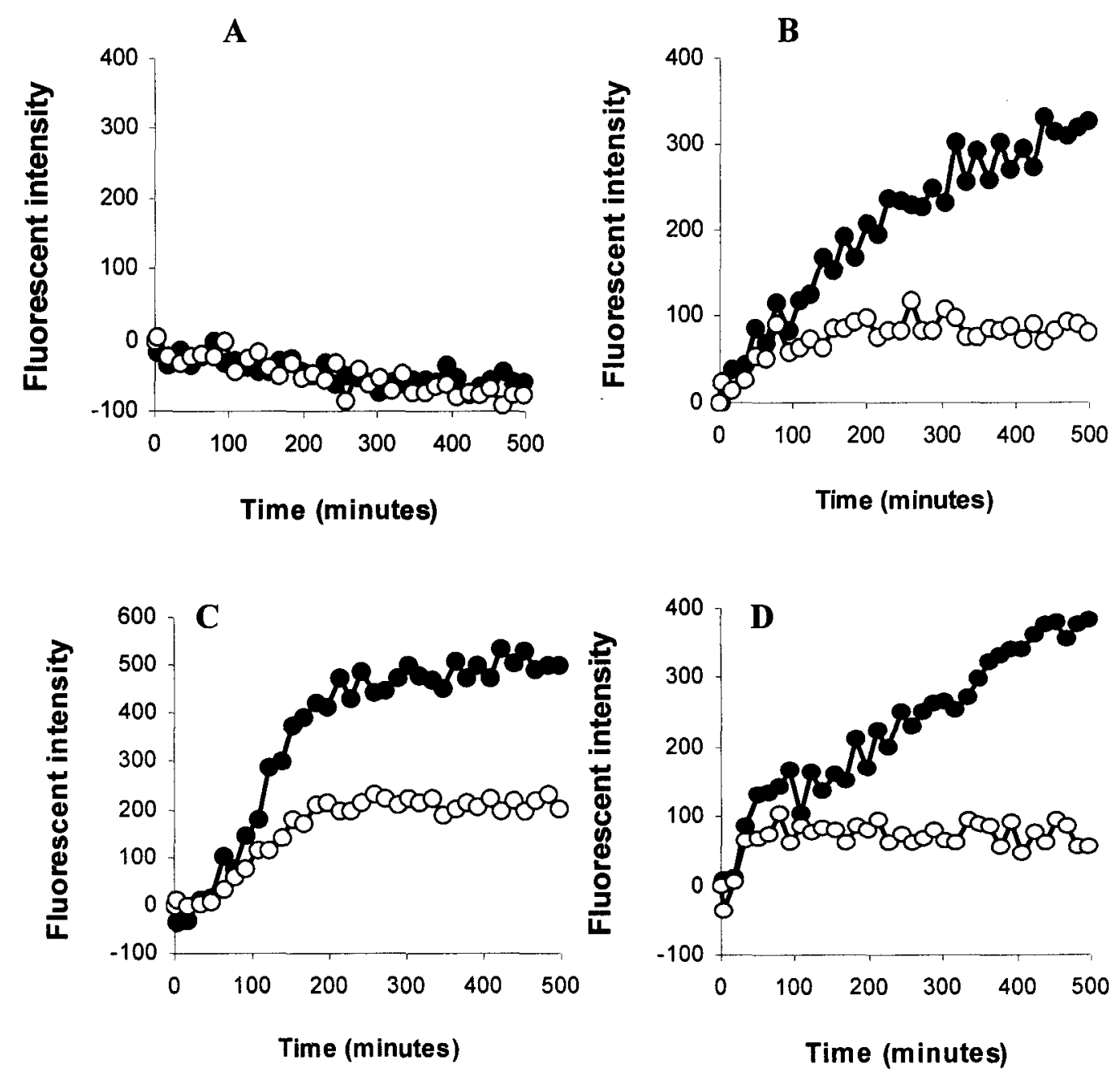

Figure 3-5. ROS production in dispersed FVB control and transgenic HMT-1 islet cells. ROS production was measured with oxidant sensitive fluorescent probe $\mathrm{CM}-\mathrm{H}_{2} \mathrm{DCFDA}$ as described in Materials and Methods. Islet cells were not treated (panel A), treated with $100 \mu \mathrm{M} \mathrm{H} 2 \mathrm{O} 2$ (panel B), treated with $30 \mu \mathrm{M} \mathrm{SIN-1} \mathrm{(panel} \mathrm{C),} \mathrm{treated} \mathrm{with} 1 \mathrm{mM}$ hypoxanthine plus $2 \mathrm{mU}$ xanthine oxidase (panel D). The solid circles indicate FVB control islet cells. The open circles indicate HMT-1 transgenic islet cells. This figure is typical of three independent experiments. This work was accomplished by Xiaoyan $\mathrm{Li}$ in our laboratory. 
A

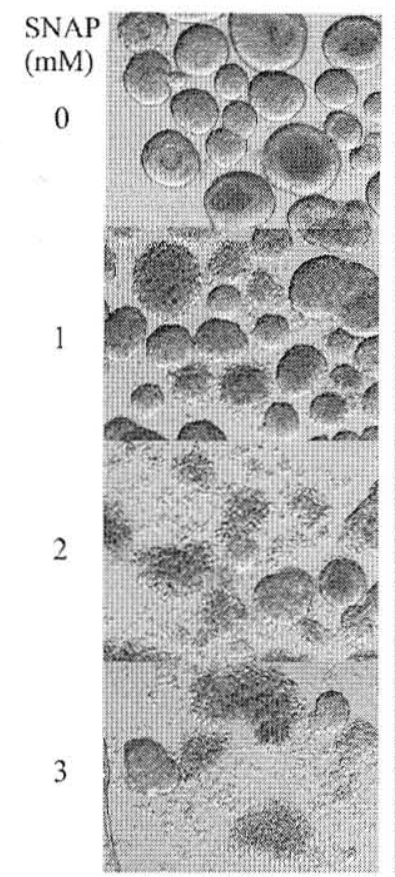

FVB

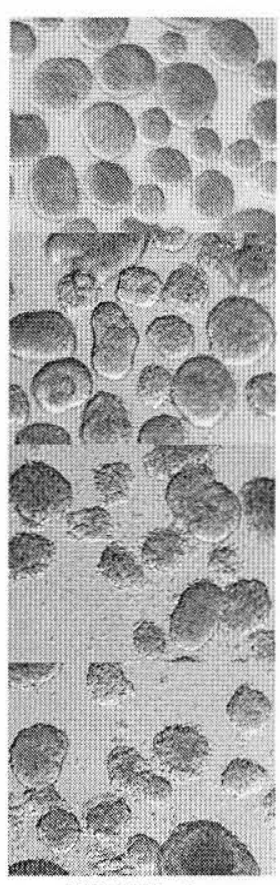

HMT-1
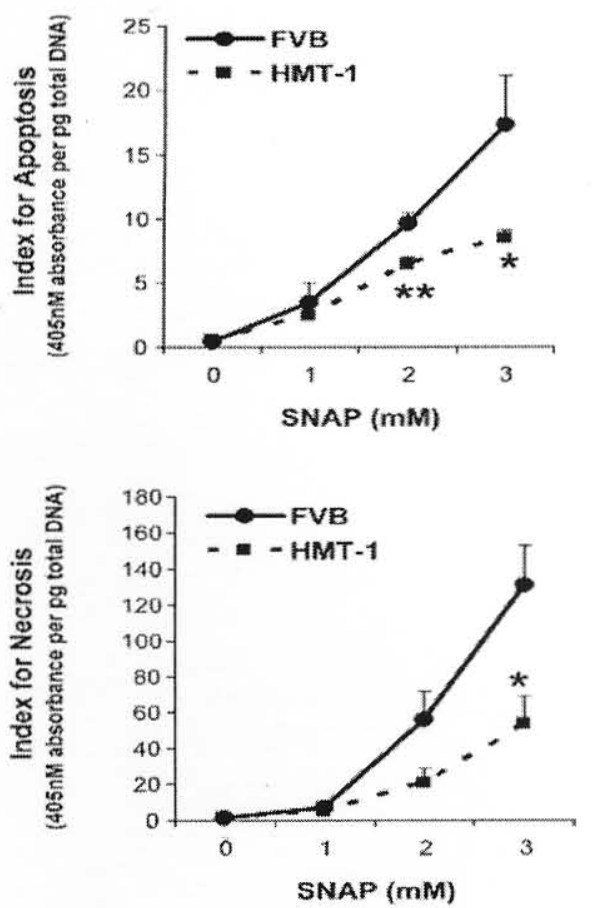

Figure 3-6. Nitric oxide induced damage in FVB and HMT-1 transgenic islets exposed to SNAP for 24 hr. (A) Representative photomicrographs of FVB and MT islets after SNAP treatment. The concentrations of SNAP are shown on the left. Similar results were obtained in four independent experiments. Magnification $\times 100$. (B) Necrosis and apoptosis in SNAP treated islets. Cell death was measured as described in Methods. Data were calculated from four independent experiments. * and ** indicate MT and FVB values were different at the same SNAP concentration $(\mathrm{P}<0.05$ and $\mathrm{P}<0.01$, respectively by one-way ANOVA test). Vertical bars indicate standard error of the mean. 
A
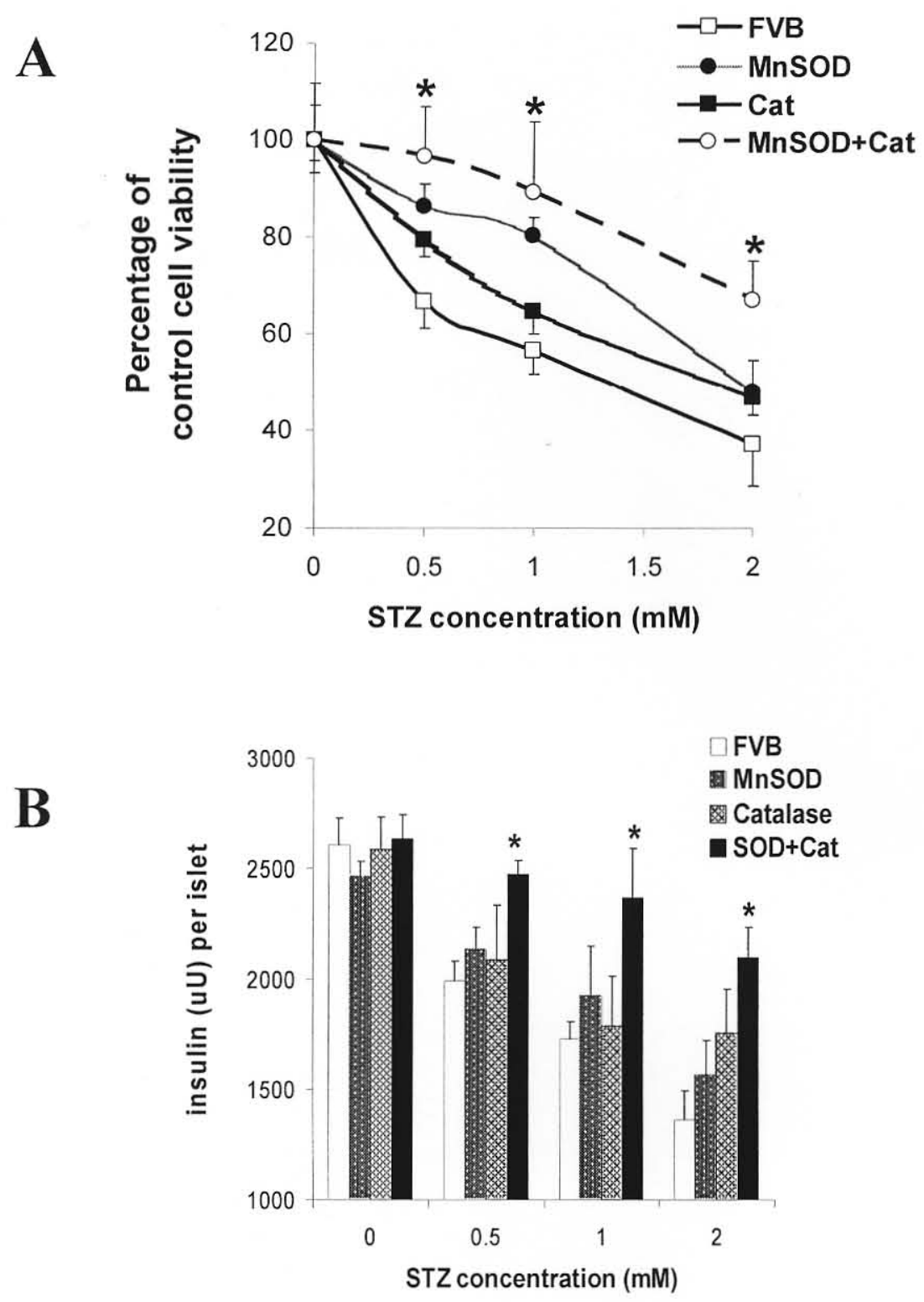

Figure 3-7. Synergistic protection by MnSOD plus Catalase against STZ induced islet cell death and insulin loss in vitro. Isolated islets were treated with the indicated concentrations of STZ for 2 days. (A) Islet cell viability measured with alamar blue assay as described in Materials and Methods. (B) Preserved islet insulin content after STZ treatment. Data were calculated from 4 independent experiments in each group. * indicates $\mathrm{P}<0.05$ between MnSOD plus Cat transgenic islets and FVB islets by two way ANOVA. Vertical bars stand for standard error of the mean. 


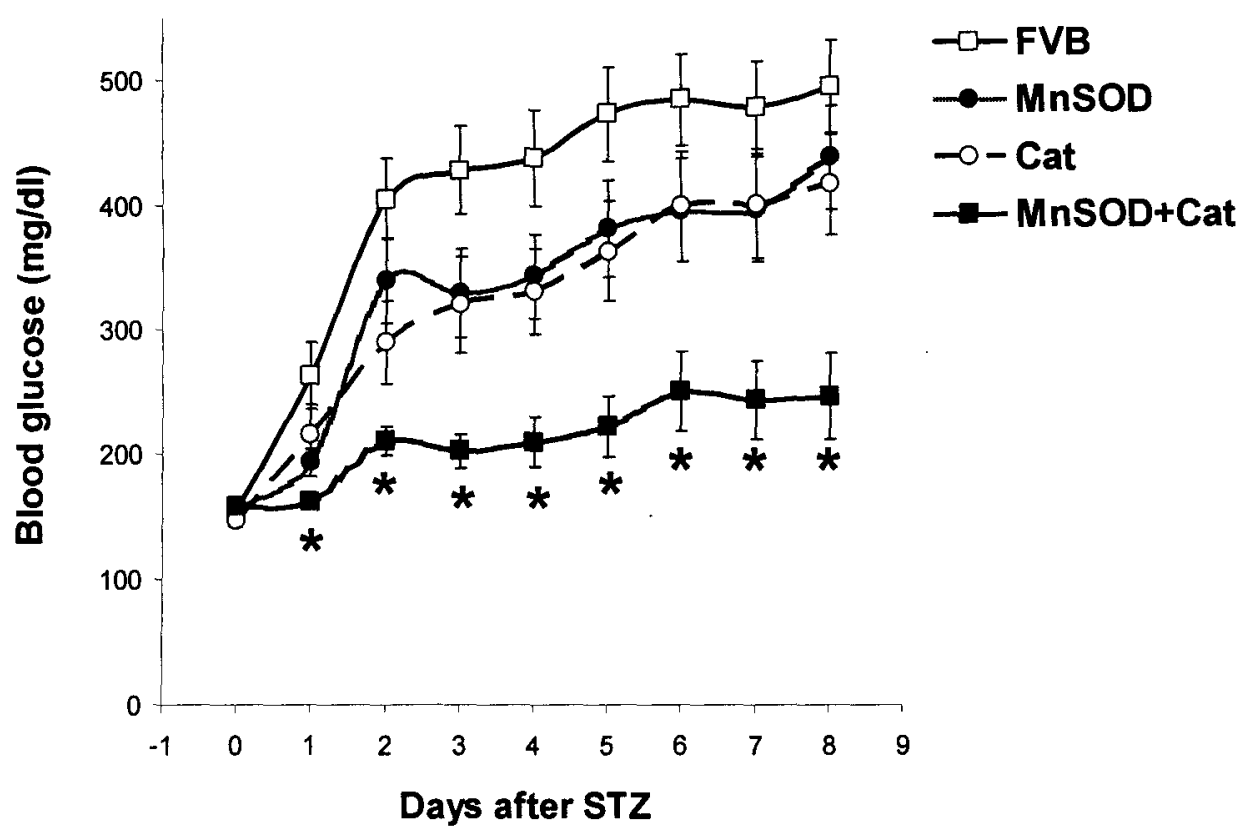

Figure 3-8. Synergistic protection by MnSOD plus Catalase against STZ induced diabetes. Sex and age matched transgenic and nontransgenic animals received a single high dose $(220 \mathrm{mg} / \mathrm{kg}$ body weight $)$ of STZ via ip injection. Blood glucose levels were monitored daily for eight days following the injection. Data shown are the mean value \pm SE from 15 or 16 animals in each group. ${ }^{*}$ indicates $p<0.01$, by two-way ANOVA for the difference between FVB and MnSOD+Cat transgenic mice at the corresponding time point. Vertical bars show the standard error of the mean. 


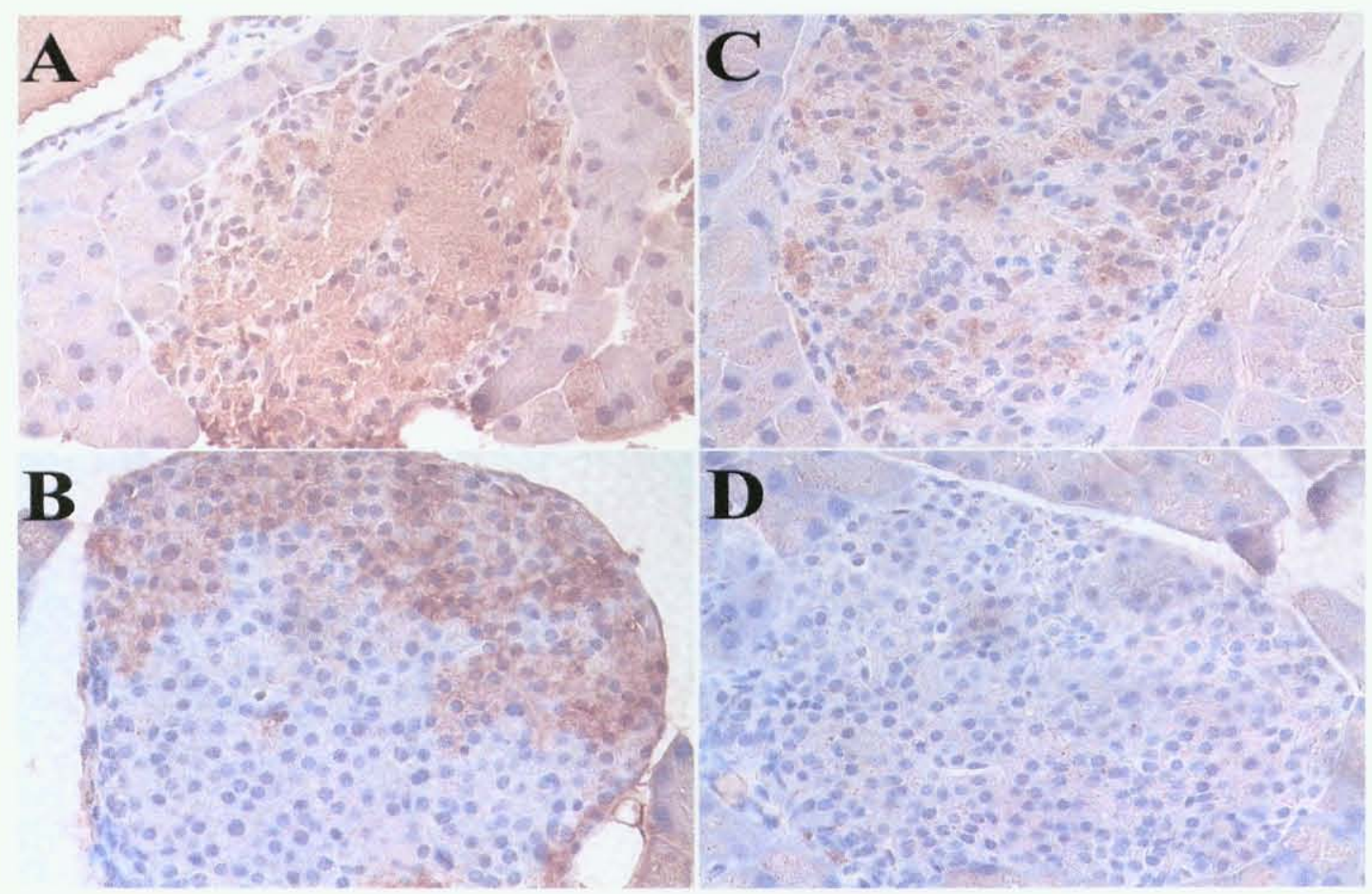

Figure 3-9. Nitrotyrosine staining in pancreatic islets from STZ treated animals. Transgenic and control mice received an intraperitoneal injection of STZ at a dose of $220 \mathrm{mg} / \mathrm{kg}$ body weight. Pancreata were removed after $30 \mathrm{hr}$ and sections were stained with a polyclonal anti-rabbit nitrotyrosine antibody. (A) FVB, (B) Cat, (C) MnSOD, (D) MnSOD+Cat. Combination of antioxidant transgene MnSOD and Catalase remarkably reduced STZ induced nitrotyrosine production in pancreatic islets. Similar results were obtained from 2 to 4 animals in each group. Magnification $\times 400$. 


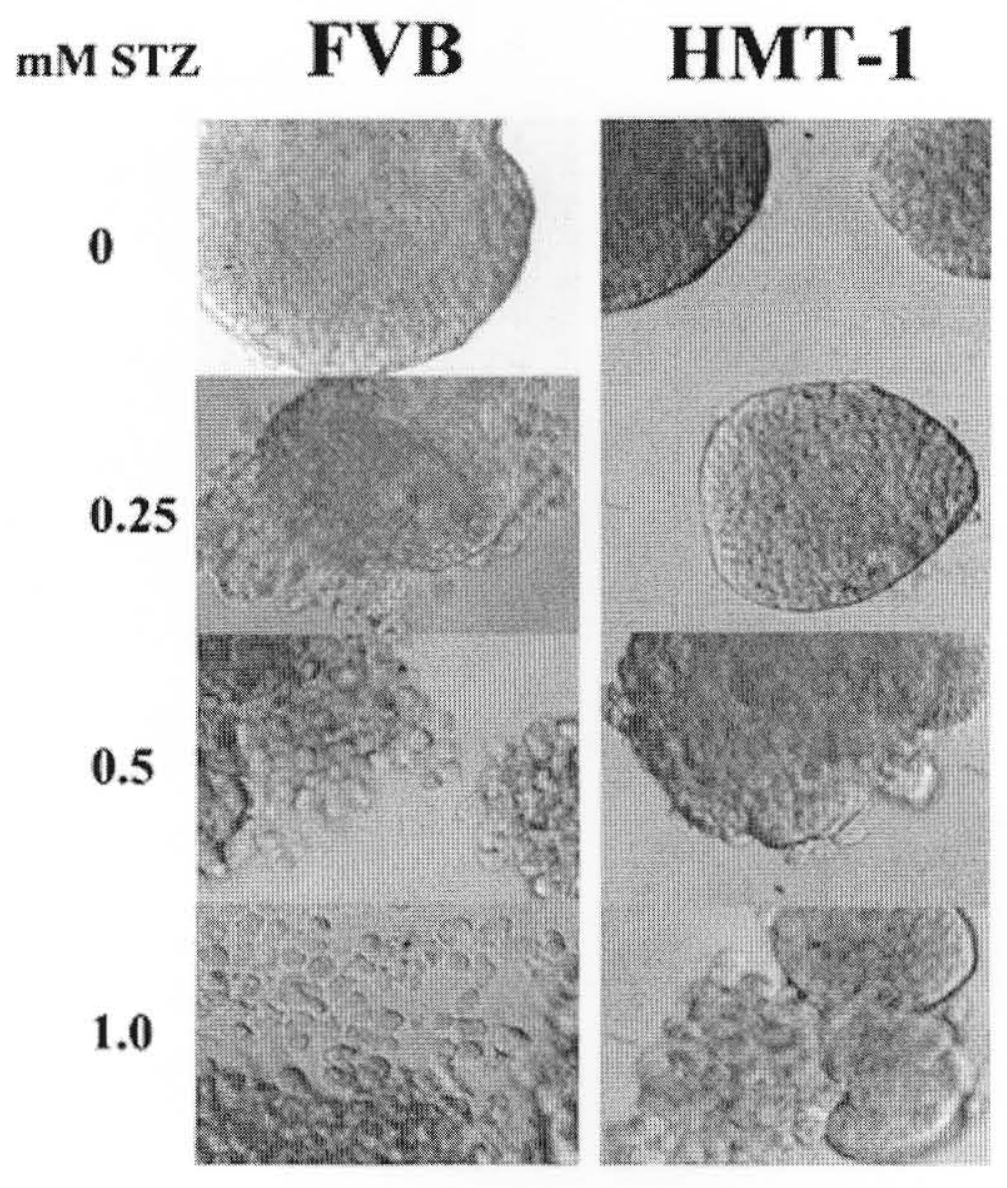

Figure 3-10. Photomicrographs of FVB and HMT-1 islets after STZ treatment in vitro. Both control and transgenic islets were treated with STZ as describe in Materials and Methods. The dose of STZ is shown on the left. In HMT-1 islets, MT overexpression reduced the disruption to morphology. Similar results were obtained in seven independent experiments. Magnification $\times 200$. 


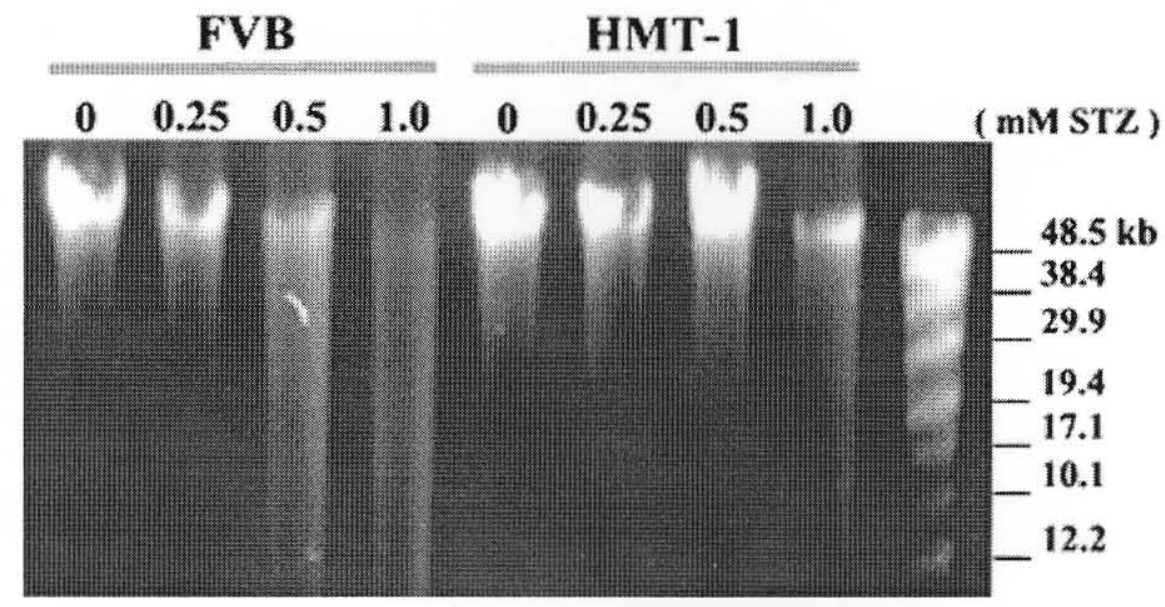

Figure 3-11. Fragmentation of high-molecular weight DNA by STZ treatment in FVB and HMT-1 islets. The MT transgene reduced DNA fragmentation. Islets were exposed to the indicated concentrations of STZ for 18 hours. DNA was analyzed as described in Materials and Methods. Similar results were obtained in seven independent experiments. 


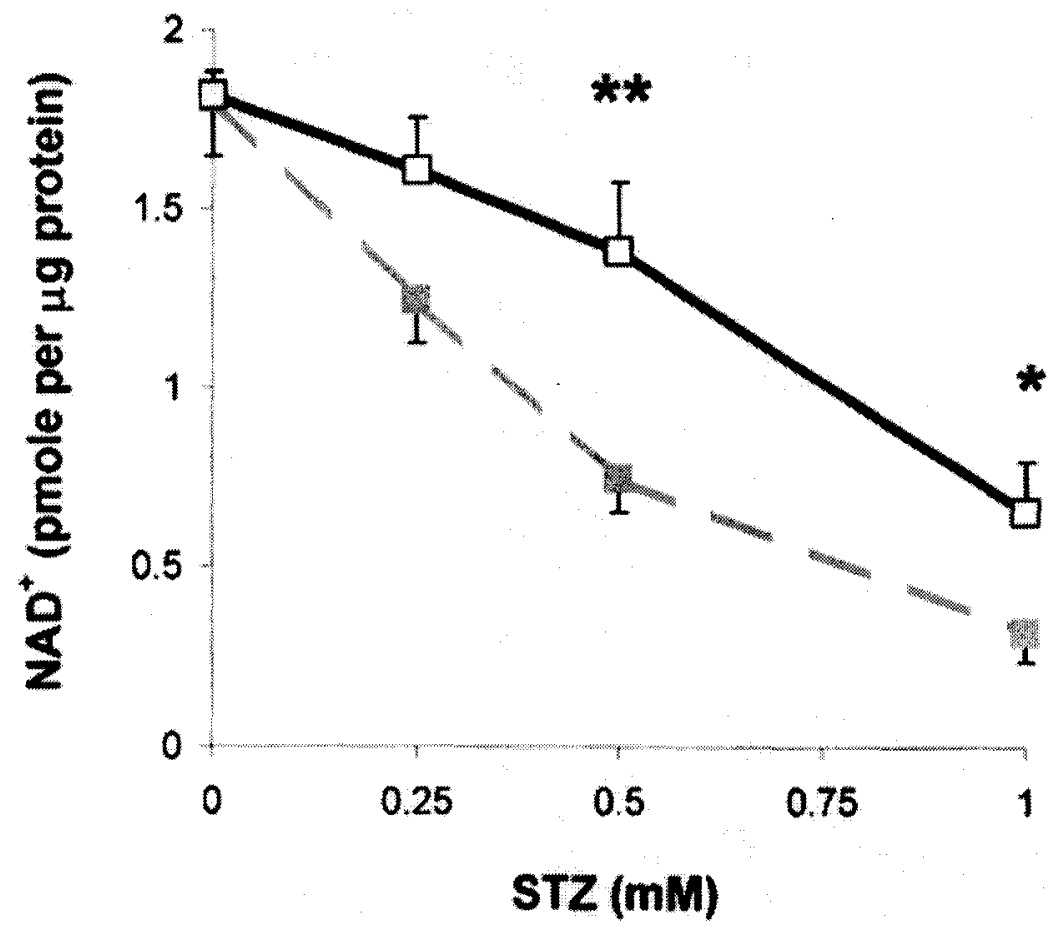

Figure 3-12. NAD ${ }^{+}$levels in FVB $(\square)$ and HMT-1 $(\square)$ islets exposed to STZ in vitro. MT overexpression protected islet $\mathrm{NAD}^{+}$from depletion by STZ. Data were calculated from five independent experiments. Where HMT-1 values are different from the corresponding FVB values, ${ }^{*} P<0.05$ and $* * P<$ 0.01 . 


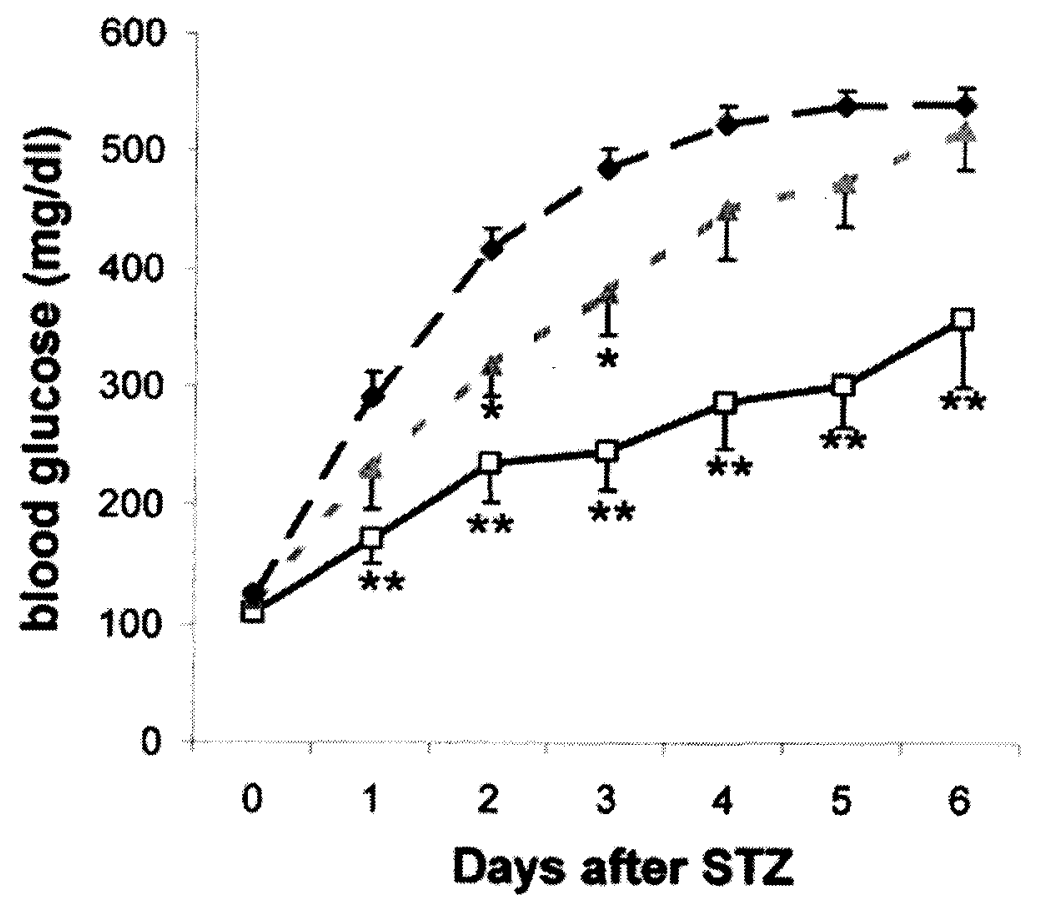

Figure 3-13. Blood glucose values measured after STZ treatment of FVB ( $)$, HMT-1 (D), and HMT-2 ( 4 ) mice. MT overexpression slows the onset of diabetes. Differences from FVB are indicated: ${ }^{*} P$ $<0.05$ and ${ }^{* *} P<0.01$ by one-way ANOVA. Vertical bars show standard error of the mean value. 

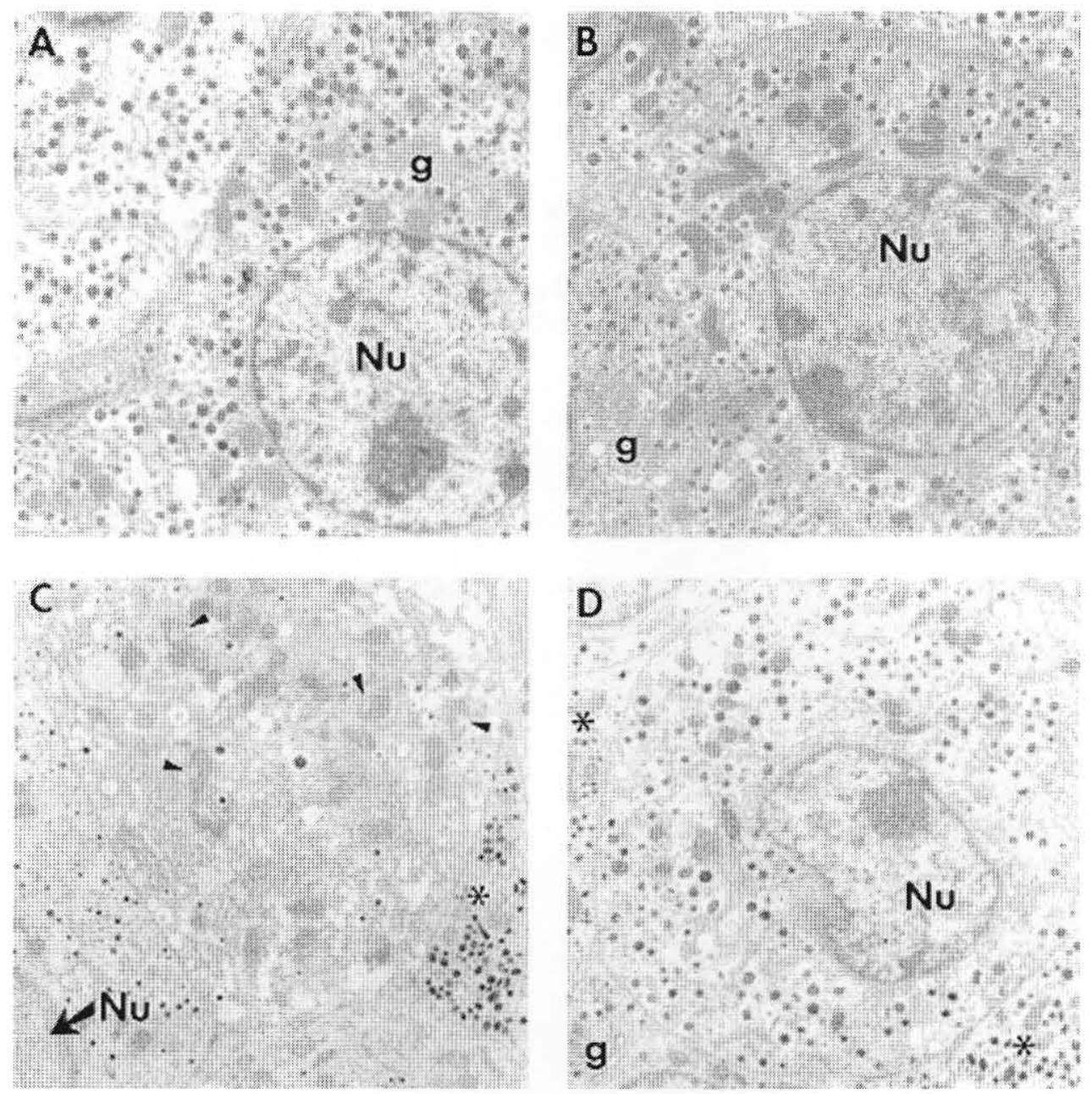

Figure 3-14. Transmission electron micrographs of isolated pancreatic FVB control and HMT-1 transgenic islets before and after STZ treatment. $A$ : Untreated islets from control (FVB) mice. $B$ : Untreated islets from transgenic (HMT-1) mice. C: STZ-treated islets from control (FVB) mice. D: STZtreated islets from transgenic (HMT-1) mice. Apparent $\beta$-cells dominate the field in all figures, but normal complements of $\beta$ granules are seen in only $A, B$, and $D$. In $C$, $B$-cells show various levels of degranulation, and the central cell in the field is dominated by vacuoles, dilated cisternae of rough endoplasmic reticulum, and "moth-eaten" mitochondria (arrow heads) in an electron-lucent cytoplasm. In all islet preparations, non- $\beta$ endocrine cells $\left(^{*}\right)$ are granule-rich and show normal morphological features. g, Golgi complex; Nu, nucleus. Magnification x 9,100 for all figures. 


\section{CHAPTER IV}

\section{THE ROLE OF ANTIOXIDANT TRANSGENES IN TOXIC EFFECT OF CYTOKINES ON PANCREATIC BETA CELLS}

\section{Introduction}

Type 1 diabetes is an autoimmune disease, characterized by selective destruction of pancreatic beta cells. In the early stage of the disease pancreatic islets are invaded with immune cells, a process termed insulitis. A large body of evidence has emerged that proinflammatory cytokines, secreted from those infiltrated macrophages and T-lymphocytes, are important mediators of beta cell functional disturbance and cell death through necrosis and apoptosis (Eizirik et al. 1996; Rabinovitch 1998). In cultured beta cells, including isolated murine, rodent and human islets, and insulin secreting cell lines, interleukin-1 $\beta$ alone or interleukin-1 $\beta$ combined with other cytokines inhibit beta cell insulin secretion, lower the synthesis of insulin, protein and DNA, and finally cause beta cell death (Corbett et al. 1993; de-Mello et al. 1996; Delaney et al. 1997; Flodstrom \& Eizirik 1997; Heimberg et al. 2001; Iwahashi et al. 1996). The detrimental effect of cytokines on beta cells is also reported in some animal models of Type 1 diabetes. In the lymphocytic choriomeningitis virus (LCMV)-induced diabetic model, LCMV induced beta cell destruction and diabetes does not occur in mice that are interferon- $\gamma$-deficient or that express a mutated interferon- $\gamma$ receptor specifically in beta cells (Oldstone et al. 1991; von et al. 1997). This indicates the importance of the cytokine interferon- $\gamma$ in the development of diabetes. Elevated beta cell expression of cytokines and cytokine receptors has been found in NOD mice that spontaneously develop autoimmune diabetes (Cardozo et al. 2003). Cytokines have also been shown to play important roles in beta cell damage in other animal models of autoimmune diabetes such as BDC2.5 T-cell receptor transgenic models (Ji et al. 1999). 
The destructive effect of cytokines on beta cells is generally recognized to be induced in part by an effect of NO that is produced by the inducible nitric oxide synthase (iNOS) upon stimulation with cytokines. Increased expression of iNOS and excessive NO production are seen in both cultured islets after treatment with cytokines and beta cell infiltrated with lymphocytes in NOD mice (Suarez-Pinzon et al. 2001) and BB rats (Kleemann et al. 1993). Application of iNOS inhibitors benefits cultured beta cells (Brandhorst et al. 2001) and prevented diabetes in a variety of animal models (Fukuda et al. 1995; SuarezPinzon et al. 2001). A target disruption of the iNOS gene provided partial protection against diabetes in mice treated with multiple low-dose STZ (Flodstrom et al. 1999). However, in some other studies cytokine induced beta cell dysfunction and cell death were shown to be independent of the NO generation pathway (Wu et al. 2001; Saldeen et al. 2001). In human islets pharmacological inhibition of iNOS activity failed to prevent cytokine induced impairment of insulin secretion (Eizirik et al. 1994b; Welsh et al. 1994a). The islets isolated from iNOS gene deficient mice still undergo apoptosis after cytokine treatment despite high resistance to cytokine induced suppression of islet function (Liu et al. 2000a). Therefore cytokine induced beta cell toxicity may involve multiple signal pathways (Saldeen et al. 2001; Saldeen et al. 2001; Andersen et al. 2000).

The partial independence of cytokine toxicity to NO may be explained, at least in part, by cytokine induced ROS generation in the beta cells. ROS and ROS derived lipid oxidation have been found in cultured rat (Rabinovitch et al. 1992) and human islets (Rabinovitch et al. 1996) after cytokine treatment. In NOD mice beta cell destruction is found to be partially mediated by reactive oxygen intermediates, including peroxynitrite (Horio et al. 1994; Suarez-Pinzon et al. 1997). Those ROS cooperatively enhance NO mediated beta cell toxicity (Tiedge et al. 1999). Therefore, some laboratories have tested the potential effect of antioxidant enzymes or compounds against cytokine toxicity to beta cells. In insulinoma RINm5F cells (Lortz et al. 2000; Tiedge et al. 1998) and INS-1 cells (Moriscot et al. 2003; Hohmeier et al. 1998) elevated antioxidant proteins, such as catalase, glutathione peroxidase, $\mathrm{Cu} / \mathrm{Zn} \mathrm{SOD}$ or MnSOD conferred protections against cytokine mediated toxicity. But their data were not completely consistent because one group reported the protection of MnSOD against interleukin-1 $\beta$ was through the reduction of NO (Hohmeier et al. 1998), presumably due to reduced iNOS expression, while the other report showed the 
protection by these antioxidants was unrelated to iNOS expression (Lortz et al. 2000). External application of antioxidant enzymes or compounds also showed conflicting results; in some studies ROS scavengers were protective against cytokine toxicity, whereas in other reports there was no protective effect (de-Mello et al. 1996; Welsh et al. 1994b). Therefore, it still remains uncertain whether antioxidant treatment is beneficial for pancreatic beta cells exposed to cytokines. Moreover, no study has been carried out to test whether the constant increase of antioxidant expression in a transgenic animals could protect primary beta cells from cytokines even though a transgenic mouse model with $\mathrm{Cu} / \mathrm{Zn}-\mathrm{SOD}$ overexpression, which is resistant to alloxan induced diabetes, was established in 1997 (Kubisch et al. 1997).

Therefore, in this part of study we used our transgenic mice to further investigate the potential effect of antioxidants, catalase, MnSOD, and MT on cytokine induced beta cell destruction in isolated primary mouse pancreatic islets. Since our antioxidants were expressed in real beta cells rather than insulinoma cell lines and the expression is permanent and within the cell, our results are not subject to many of the limitations of earlier studies. Our result showed that none of these antioxidants conferred any benefit at all to pancreatic mouse islets after exposure to cytokines. 


\section{Materials and Methods}

Islet insulin secretion after cytokine treatment: Cytokine treatment and followed insulin secretion assay were carried out on 96-well tissue-culture plates. In each well 10 islets were incubated in $200 \mu \mathrm{I}$ culture medium in absence or presence of different concentrations of cytokines (mouse recombinant interleukin-1 $\beta$ plus interferon- $\gamma$, Sigma, St. Louis, MO) for 18 hours. After incubation, islets were rinsed with $3 \mathrm{mM}$ glucose modified KRB buffer twice. Static islet insulin secretion was performed by stimulation in $20 \mathrm{mM}$ glucose modified $\mathrm{KRB}$ solution, and secreted insulin level was measured with the procedure as stated in MATERIALS AND METHODS OF CHAPTER II (Page 25).

Islet cell viability after cytokine treatment. Islet cell viability was indicated by islet cell metabolic activity that was measured with Alamar Blue, as described in MATERIALS AND METHODS OF PART II. In detail, fifteen overnight-cultured islets were hand picked into $200 \mu \mathrm{l}$ fresh culture medium (no phenol red) containing 1:20 diluted Alamar Blue in a 96-well microplate. A pre-treatment 4-hr metabolic activity of the islets was measured for normalizing the post-treatment islet metabolic activity. After three washes of fresh culture medium (no phenol red), islets were cultured for six days in $200 \mu$ l culture medium (no phenol red) supplemented with or without varying concentrations of cytokines (mouse recombinant interleukin- $1 \beta$ plus interferon- $\gamma$ ). Every other day $100 \mu$ culture medium was replaced with $100 \mu$ fresh culture medium (no phenol red) containing cytokines, thereby no change of final cytokine levels. In the end of treatment, $50 \mu \mathrm{l}$ culture medium was replaced with $50 \mu \mathrm{l}$ fresh culture medium containing 1:5 diluted Alamar Blue, for a final dilution of 1:20. The color was developed for another $4 \mathrm{hr}$ and the fluorescence was measured again. A fluorescent value from the "blank well", which contained no islets but exactly followed the above procedure with highest level of cytokine treatment, was deducted from all other fluorescent values. Islet cell viability was calculated as the ratio of fluorescence after treatment to the fluorescence before treatment. The untreated islet cell viability was arbitrarily set as $100 \%$.

Measurement of nitrite formation: Nitrite formation was measured in islet culture medium as an indication of cytokine-induced NO production, as previously described (Hohmeier et al. 1998). The 
treatment of cytokines was performed on 96-well tissue-culture plates. For each well 40 to 50 overnightcultured islets were cultured for 24 hours in $170 \mu$ islet culture medium (no phenol red) with or without cytokines (mouse recombinant interleukin- $1 \beta$ plus interferon- $\gamma$ ). After incubation, the culture media were collected, and $150 \mu \mathrm{l}$ of culture medium was reacted with $150 \mu \mathrm{l}$ of Griess reagent (Molecular Probes, Eugene, OR) at room temperature for $30 \mathrm{~min}$. The optical density was read at $550 \mathrm{~nm}$ using a Tecan (Durham, NC) plate reader. Nitrite concentration was calculated using a sodium nitrite (Sigma, St. Louis, MO) standard curve prepared in islet culture medium (no phenol). Data were normalized to total islet DNA determined by picogreen DNA assay (Molecular Probes, Eugene, OR).

Islet iNOS expression after cytokine treatment. The messenger RNA level of iNOS in cultured islets was measured by real-time quantitative RT-PCR. Eighty to one hundred overnight-cultured islets were treated without or with cytokines (mouse recombinant interleukin- $1 \beta 5 \mathrm{U} / \mathrm{ml}$ plus interferon- $\gamma 100$ $\mathrm{U} / \mathrm{ml}$ ) in $300 \mu \mathrm{l}$ culture medium for six hours in $1.5 \mathrm{ml}$ microtubes. After treatment islets were rinsed with fresh HBSS solution twice and islet total RNA was extracted with an RNA preparation kit from Stratagene (Absolutely RNA Microprep Kit, Stratagene, La Jalla, CA) according to the manufacturer instructional manual. The RNA sample was treated with DNase to remove all traces of genomic DNA contamination and its concentration was determined by Ribogreen RNA quantification kit (Molecular Probes, Eugene, OR). Fifty ng total islet RNA was reverse-transcribed to cDNA with a StrataScript reverse transcriptase (Stratagene) in the presence of oligo dT based on the manufacturer provided procedure. Real time quantitative PCR was prepared using Brilliant ${ }^{\mathrm{TM}}$ plus Two-Step Quantitative RT-PCR Core Reagent kit (Stratagene) and executed using Mx4000 ${ }^{\mathrm{TM}}$ real-time multiplex quantitative PCR system (Stratagene). The cDNA sequences for murine $\beta$-actin and iNOS were obtained from GenBank, and the primer and probe sequences were designed using Primer3 software (Steve Rozen and Helen J. Skaletsky (2000) Primer3 on the WWW for general users and for biologist programmers. In: Krawetz S, Misener S (eds) Bioinformatics Methods and Protocols: Methods in Molecular Biology. Humana Press, Totowa, NJ, pp 365-386). To avoid amplification of genomic DNA, the primer-probe sets were designed to span exon-exon boundaries. The probes were labeled with a fluorescent reporter 6-carboxyfluorescin (FAM) at the 5' end and a quencher, 
6-carboxytetramethylrhodamine (TAMRA) at the 3' end. The sequences for the primer-probe sets for each gene were as follows: iNOS (forward 5'-GTAGCCAGCGTACCGGATGA-3', reverse 5'CAACATCAGGTCGGCCATCAC-3', probe 5'- CCCCAGCGGACTGACGGCAAACAT-3'); $\beta$-actin (forward 5'-ATGGCTGGGGTGTTGAAGGT-3', reverse 5'-TACAATGAGCTGCGTGTGGC-3', probe 5'- CACCCTGTGCTGCTCACCGAGGC-3'). PCR amplifications were performed in a total volume of 25 $\mu \mathrm{l}$, containing $1 \mu \mathrm{l}$ of cDNA sample (transcribed from $\sim 50 \mathrm{ng}$ total islet RNA), $900 \mu \mathrm{M}$ of each primer, $250 \mu \mathrm{M}$ of the corresponding probe, $2.0 \mu \mathrm{l}$ of GAUC mix $(200 \mu \mathrm{M}$ each of dATP, dCTP and dGTP, but $400 \mu \mathrm{M}$ of dUTP), $0.01 \mathrm{U} / \mu \mathrm{l}$ Uracil-DNA-N-glycosylase (UNG), $0.05 \mathrm{U} / \mu \mathrm{l}$ of SureStart Taq DNA polymerase, $1 \times$ core $\mathrm{PCR}$ buffer, $5.5 \mathrm{mM} \mathrm{MgCl}_{2}$. For each reaction, $\mathrm{UNG}$ reaction was processed at $50^{\circ} \mathrm{C}$ for $2 \mathrm{~min}$ followed a polymerase activation step at $95^{\circ} \mathrm{C}$ for $10 \mathrm{~min}$, then amplification was executed by alternating between $95^{\circ} \mathrm{C}$ for $15 \mathrm{~s}$ and $60^{\circ} \mathrm{C}$ for $60 \mathrm{~s}$ for 40 cycles. The sample iNOS and $\beta$-action mRNA levels were calculated respectively from standard curves of iNOS and $\beta$-actin using FVB control islets exposure to mouse recombinant interleukin- $1 \beta 50 \mathrm{U} / \mathrm{ml}$ plus interferon- $\gamma 1000 \mathrm{U} / \mathrm{ml}$ for 6 hours. Data were expressed as folds of iNOS expression over $\beta$-actin expression.

Data analysis: Data are presented as the mean \pm standard error. Statistical analysis was performed by one-way or two-way ANOVA and Dunnet's post hoc (2-tailed) test. Computations were carried by statistical programs from SPSS (version 10.0) and Sigmastat (version 2.03). 


\section{$\underline{\text { Results }}$}

Neither MnSOD nor catalase protected pancreatic islets from cytokine cytotoxicity. It is well established that inflammatory cytokines suppress pancreatic beta cell insulin secretion and lead to cell death through necrosis and apoptosis (Eizirik et al. 1996; Eizirik \& Darville 2001). In this study both beta cell function of insulin secretion and cell viability were assessed in cultured control FVB and transgenic islets after exposure to cytokines. As shown in Figure 4-1, cytokine treatment (interleukin-1 $\beta$ plus interferon- $\gamma$ ) caused a drastic inhibition of islet insulin secretion. However neither the MnSOD nor catalase transgene reversed this suppression at any concentration of cytokine tested. Instead of having synergistic protection against STZ and peroxynitrite as demonstrated in Part II, combination of MnSOD and catalase did not provide pancreatic islets with any resistance to cytokine induced beta cell toxicity as revealed by both islet insulin secretion (Figure 4-1A) and islet metabolic activity (Figure 4-1B).

Cytokine mediated destruction of pancreatic beta cells is accompanied by activation of iNOS expression and production of NO. MnSOD and antioxidant compounds have been shown to inhibit iNOS activation and NO production in insulin secreting cell lines after cytokine treatment. Therefore we further measured iNOS expression levels by real time quantitative RT-PCR and NO production by the Griess reaction in islets exposed to cytokines. None of the beta cell antioxidant transgenes, including MnSOD, catalase or MnSOD plus catalase was able to decrease cytokine induced activation of iNOS expression (Figure 4-2) and NO production (Figure 4-3) at every concentration of cytokines we examined. These data indicate no protective effect of MnSOD and catalase against cytokine cytotoxicity in primary mouse beta cells.

MT did not protected pancreatic islets from cytokine cytotoxicity. We carried out similar studies on MT transgenic islets exposed to cytokines. MT seemed a promising candidate for protection from cytokines since MT is a potent NO radical scavenger as demonstrated by our own and other laboratories (Cai et al. 2000; Kennedy et al. 1993). However, our data showed that the MT transgene also failed to provide protection against cytokine induced beta cell toxicity. After cytokine treatment the inhibition of 
glucose stimulated insulin secretion (Figure 4-4) and stimulation of NO production (Figure 4-5) were the same in control FVB and HMT-1 transgenic islets. Therefore, our data suggest, unlike the data from insulin secreting tumor cell lines, that ROS may not mediate cytokine induced cytotoxicity in real beta cells. 


\section{$\underline{\text { Discussion }}$}

In this part of study, we investigated the potential role of antioxidant transgenes against cytokine toxicity to pancreatic islet cells. The data showed that none of the antioxidant transgenes we tested, including MnSOD, catalase, MnSOD plus catalase, and MT, was able to protect from cytokine toxicity revealed by measurements of glucose stimulated islet insulin secretion, islet cell metabolic activity, iNOS expression and NO production in culture islets exposure to cytokines. Our results imply that antioxidant proteins do not alter cytokine toxicity in primary mouse beta cells.

Proinflammatory cytokines are important destructive mediators causing pancreatic beta cell death in various species, including human, rat and mouse (Eizirik et al. 1994b; Cardozo et al. 2001; Eizirik et al. 1994b; Rabinovitch et al. 1992; Utsugi et al. 1996). A variety of studies have demonstrated that IL-1 $\beta$ alone is able to provoke dysfunction of beta cells and finally lead to cell death (Ling et al. 2000). The effect of $I L-1 \beta$ is potentiated by combination with other cytokines such as interferon- $\gamma$ and tumor necrosis factor$\alpha$ (Eizirik et al. 1996). Induction of MnSOD, heat shock protein 70, and heme oxygenase and iNOS are commonly seen in pancreatic beta cells exposed to cytokines (Andrade et al. 1996; Ling et al. 2000; Strandell et al. 1995). As a consequence of iNOS induction intracellular production of NO is greatly increased. NO is considered to be a major culprit leading to beta cell death, although there are several studies showing NO is not involved in beta cell apoptosis after cytokine treatment (Liu et al. 2000a; Ling et al. 2000; Saldeen et al. 2001). In addition, experimental evidence indicates that ROS are generated in cytokine-stimulated beta cell via unknown mechanisms (Horio et al. 1994; Rabinovitch et al. 1992). Therefore studies have been performed to show that overexpression of antioxidants in insulin secreting cell lines resulted in a significantly higher resistance against cytokines and NO mediated cytotoxicity. Stable overexpression of MnSOD in INS-1 and RIN1046-38 cells (Hohmeier et al. 1998) provided complete protection against IL-1 $\beta$ toxicity via the inhibition of NO production. In RIN5mF cell lines, overexpression of catalase, glutathione peroxidase and $\mathrm{Cu} / \mathrm{Zn}$ SOD prevents cell viability loss caused by cytokine mixtures (Azevedo-Martins et al. 2003; Lortz et al. 2000; Tiedge et al. 1999). However our results totally disagreed with these results showing protection of beta cell lines from cytokines by antioxidants. 
The reason for the discrepancy between our results and other labs results is uncertain. The simplest explanation is that our primary mouse beta cells (real beta cells) have a different response pathway to cytokine induced damage than exists in beta cell tumor lines (abnormal beta cells). Our results indicate that ROS are not involved in the cytokine response of "real", primary beta cells. The results of other labs indicate that ROS are involved in the response of "artificial" tumor beta cell lines. However, this interpretation could be overly simplistic. Recent studies, consistent with some of our results in the next section, suggest that ROS are involved not only in cell destruction but also in activation of cell protective pathways (Kamata \& Hirata 1999; Forman et al. 2002). Thus an alternative explanation for our failure to see protection from cytokines by antioxidants is that our antioxidant transgenes prevent cell protective pathways from being turned on by ROS. Our antioxidant response could be a combination of ROS protection from cytokines and inhibition of cell protection pathways. The net effect may be that we saw no benefit of antioxidants in the preceding studies. At this time this is of course, speculative. 


\section{A}

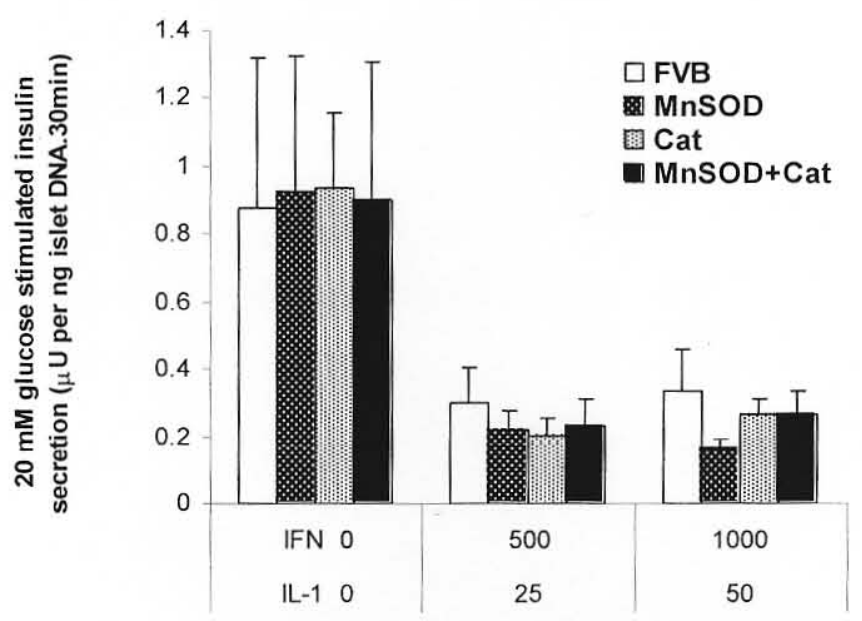

Cytokine concentrations (Unit/ml)
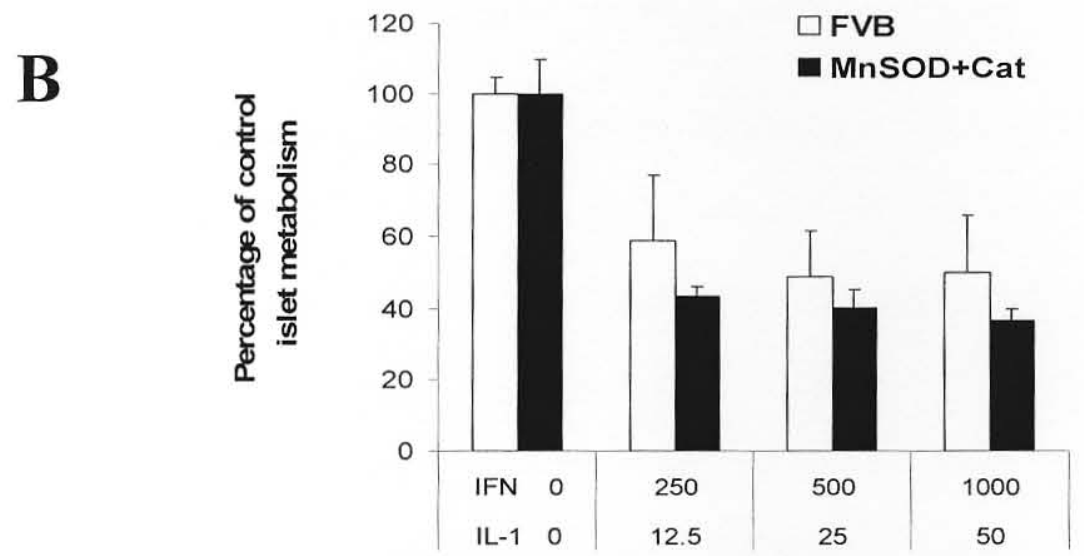

Cytokine concentrations (Unit/ml)

Figure 4-1. Glucose stimulated insulin secretion and cell viability in control FVB and transgenic islets after cytokine treatment. (A) Isolated islets were treated with indicated concentrations of interleukin-1 $\beta$ (IL-1) and interferon- $\gamma$ (IFN) for 18 hours. Basal (3mM glucose, data not shown) and $20 \mathrm{mM}$ glucose stimulated islet insulin secretion were measured with coated tube RIA kit as described in the Methods. (B) Cell viability in cultured islets after 6 days of cytokine treatment. Cell viability was measured by Alamar Blue assay. Data were calculated from four independent measurements of two experiments in each group. Neither single transgene (MnSOD or Catalase) nor combination of MnSOD and Catalase prevented from cytokine induced islet cell insulin secretion dysfunction and cell death. Vertical bars show the standard error of the mean. 


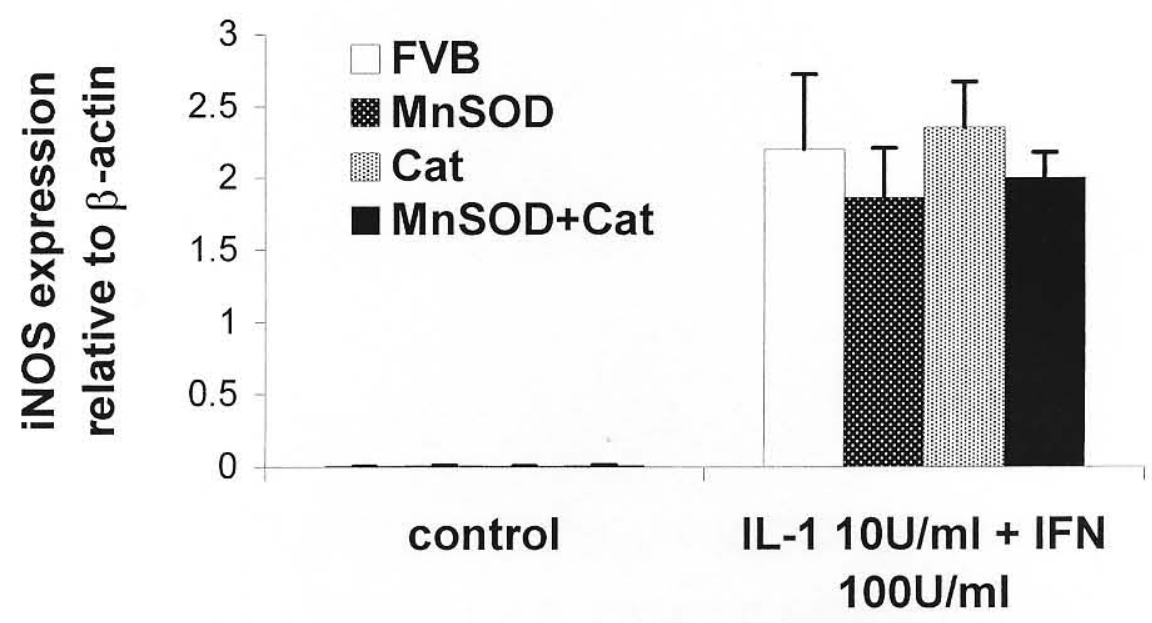

Figure 4-2. Inducible nitric oxide synthase (iNOS) expression in transgenic and control islets after treatment with cytokines. Isolated islets were treated with $10 \mathrm{U} / \mathrm{ml}$ interleukin- $1 \beta$ (IL-1) and $100 \mathrm{U} / \mathrm{ml}$ interferon- $\gamma$ (IFN) for 6 hours. Total RNA was extracted from islets. iNOS expression was measured with real-time quantitative RT-PCR and normalized with islet $\beta$-actin expression. Either single transgene (MnSOD or Catalase) or combination of MnSOD and Catalase was unable to suppress cytokine-induced iNOS activation in the islets. Vertical bars show the standard error of the mean. 


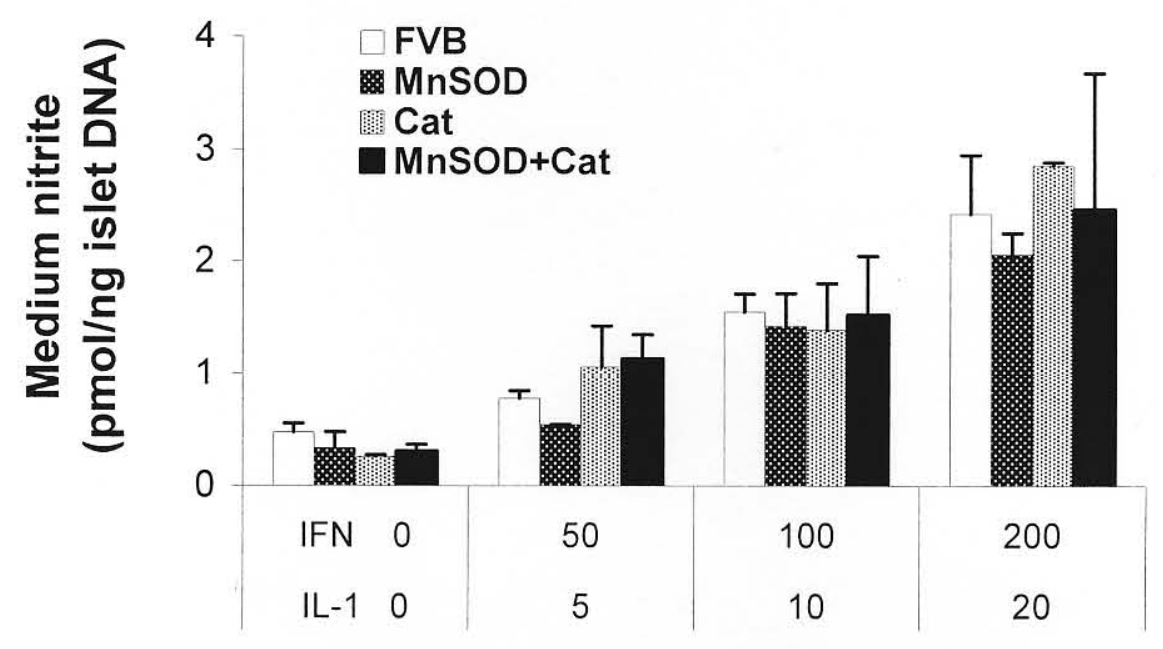

Cytokine concentrations (Unit/ml)

Figure 4-3. Nitric oxide release in transgenic and control islets after treatment with cytokines for 24 hours. Isolated islets were treated with indicated conentrations of IL-1 $\beta$ and IFN- $\gamma$. The level of nitrite released into the culture medium was measured by Greiss agent. Values were normalized with islet DNA content. Data were calculated from four independent experiments in each group. Either single transgene (MnSOD or Catalase) or combination of MnSOD and Catalase was unable to suppress cytokine-induced NO production in the islets. Vertical bars show the standard error of the mean. 


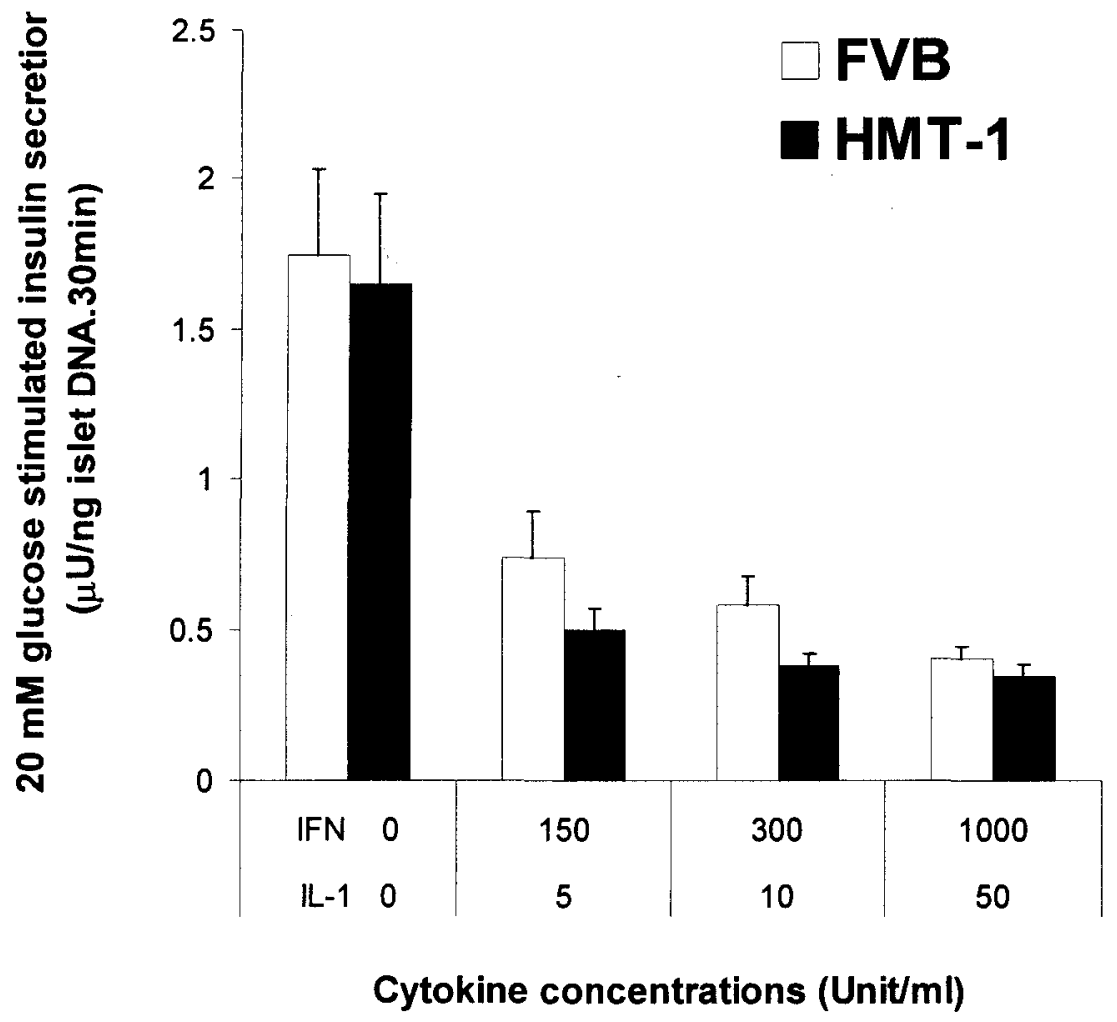

Figure 4-4. Glucose stimulated insulin secretion in FVB control and HMT-1 transgenic islets after cytokine treatment. Isolated islets were treated with indicated concentrations of interleukin-1 $\beta$ (IL-1) and interferon- $\gamma$ (IFN) for 18 hours. Basal (3mM glucose, data not shown) and $20 \mathrm{mM}$ glucose stimulated islet insulin secretion were measured by coated tube RIA kit as described in the Methods. Data were calculated from 8 to 12 independent measurements at every concentration of cytokines in each group. MT transgene did not block the inhibition of islet insulin secretion induced by cytokines. Vertical bars show the standard error of the mean. 


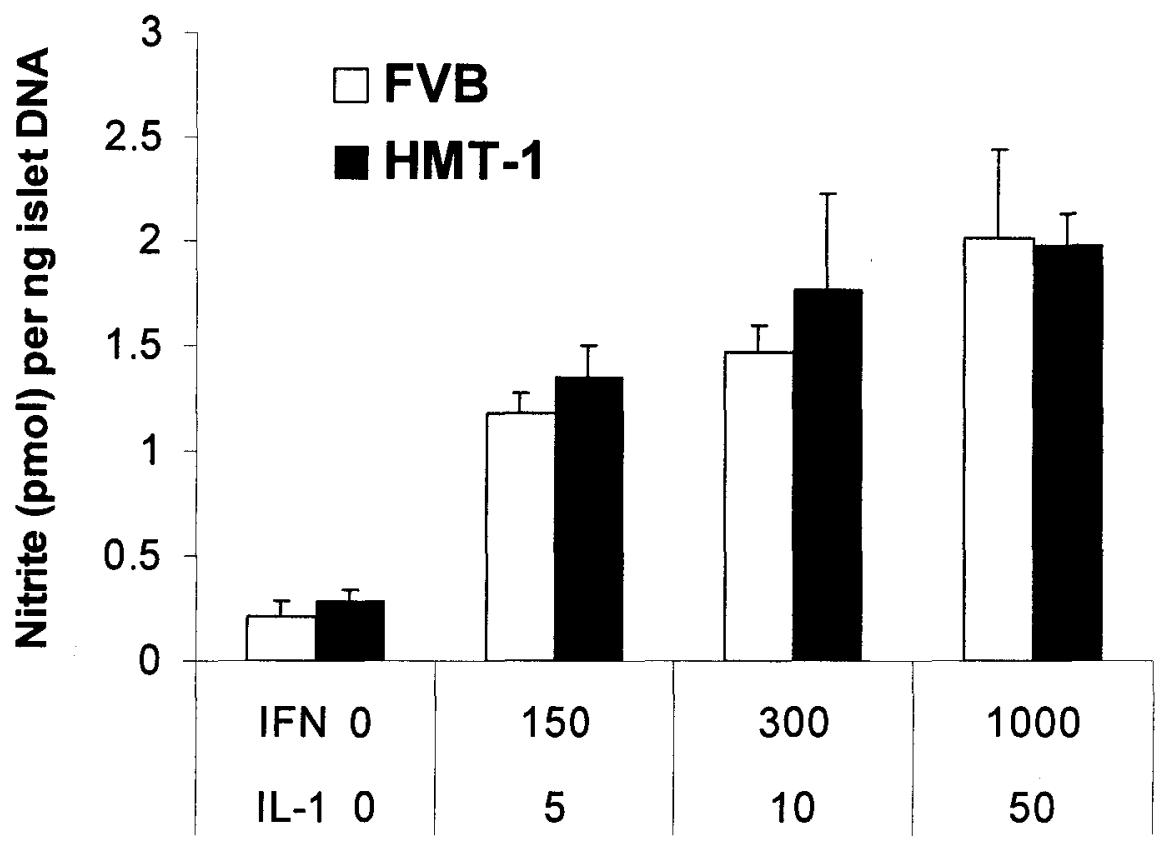

Cytokine concentrations (Unit/ml)

Figure 4-5. Nitric oxide release in HMT-1 transgenic and control FVB islets after treatment with cytokines. Isolated islets were treated with indicated concentrations of IL-1 $\beta$ (IL-1) and IFN- $\gamma$ (IFN) for 24 hours. The level of nitrite released into culture medium was measured by Greiss agent. Data were calculated from four independent experiments in each group. MT did not suppress cytokine-induced NO production in the islets. Vertical bars show the standard error of the mean. 


\section{CHAPTER V}

\section{THE ROLE OF ANTIOXIDANT TRANSGENES IN TYPE 1 DIABETES OF NOD MICE}

\section{Introduction}

Several models are available to study human Type 1 diabetes. NOD mice and Bio-Breeding (BB) rat spontaneously develop autoimmune diabetes (Leiter et al. 1987b; Maclaren et al. 1983). Another model, multiple low dose administration of STZ model (Shimizu et al. 1991) is the most rapid and convenient way to produce autoimmune diabetes. In this model animals receive up to five STZ injections at a very low dosage (40 to $60 \mathrm{mg} / \mathrm{kg}$ ) (Flodstrom et al. 1999). Such low doses of STZ initially cause a limited damage to pancreatic beta cells, which activates the immune system and triggers a late, massive immune destruction of beta cells, resulting in autoimmune diabetes. However, this model is inappropriate in our project since all of our antioxidant transgenes; MnSOD, catalase and MT are able to protect from STZ. Due to this protection it becomes impossible for us to discriminate whether the potential protection from multiple low doses of STZ by catalase, MnSOD or MT comes from the protection against immune attack or from the direct protection against STZ itself. Therefore, in this study the NOD mouse model for Type 1 diabetes was chosen for investigation of the potential effects of antioxidant transgenes against Type 1 diabetes.

NOD mice have been accepted as one of the best models of human autoimmune Type 1 diabetes. In this strain, both female and male NOD mice develop diabetes spontaneously, but the females are more susceptible. In the female mice the incidence of diabetes is $90-100 \%$ by 30 weeks of age. Male mice develop diabetes at a frequency of 40 to $60 \%$ by $30-40$ weeks of age (Leiter et al. 1987b). This strain of mice was first reported in 1980 (Makino et al. 1980) and now it has been recognized to have very similar features to human Type 1 diabetes. Before becoming overtly diabetic, with non-fasting plasma glucose higher than $250 \mathrm{mg} / \mathrm{dl}$, NOD mice go through a long, clinically silent period characterized by a leukocytic 
infiltrate of the pancreatic islets, referred to as insulitis. This insulitis phase is also present in prediabetic human patients. As early as 12 weeks of age a marked reduction of pancreatic insulin contents occurs in female mice, and a few weeks later in males, due to a selective destruction of pancreatic islet beta cells. The destruction to beta cells is a progressive process mediated by autoimmune reaction involving inflammatory cells, cytokines, and autoantibodies. All of these processes are very similar to human Type 1 diabetes (Leiter et al. 1987b; McAleer et al. 1995).

Susceptibility to Type 1 diabetes in NOD mice is polygenic. Currently at least 20 diabetic susceptible alleles have been identified that are associated with the onset and development of diabetes in the NOD mouse (Adorini et al. 2002). These alleles are highly polymorphic. This makes it possible to identify the diabetic susceptible alleles with the aid of microsatellite markers, a group of variable DNA sequences closely linked to the diabetic susceptibility loci. By genotyping these diabetic susceptible alleles, Serreze $e t$ al. (Serreze et al. 1996) established a successful protocol for producing "speed congenic NOD mice" from other strains of mice. In his procedure, a transgenic FVB mouse was continuously backcrossed to NOD mice for four generations. From the fourth generation, the transgenic litters were assessed for homozygosity of 15 diabetic susceptible alleles by standard PCR using a series of microsatellite markers. Serreze et al. found that five backcrosses were sufficient to obtain one animal homozygous for all of 15 diabetic susceptible alleles. This animal was used as the progenitor to breed with NOD mice to produce offspring with greater identity to NOD mice. Since our antioxidant transgenic mice were established and maintained on the FVB background strain, we utilized Serreze's procedure to more rapidly generate new NOD strains homozygous for all NOD susceptibility alleles which were congenic for our pancreatic beta cell antioxidant transgenes.

The susceptibility to Type 1 diabetes in NOD mice is also significantly affected by environmental factors, including housing conditions, health status, diet and chemical or virus exposure. The onset of diabetes in NOD mice is relatively slow and random. The onset of diabetes in NOD mice is known to be highly variable from one mouse to another. Therefore cyclophosphamide (CYP), an alkylating cytostatic drug is often employed to speed up diabetes onset in NOD mice (Harada \& Makino 1984). The mechanism 
for this accelerated diabetes by CYP is not completely defined but in some studies CYP has been shown to activate immune response in NOD mice by suppressing T-helper cells (Yasunami \& Bach 1988). In this project we injected CYP into our transgenic NOD mice to accelerate and synchronize NOD diabetes.

Some others laboratories have used NOD mice to show the protective effect of antioxidant compounds in prevention of diabetes (Fukuda et al. 1995; Hotta et al. 1998). But the results from those studies are ambiguous since the antioxidant chemicals they used have multiple actions and have no specificity to the beta cell. The beneficial effect exerted by those antioxidants against NOD diabetes could come from actions on other organs, such as immune system, rather than a direct protective action on beta cells. One other antioxidant protein, thioredoxin (Hotta et al. 1998), has been specifically expressed in pancreatic beta cells. This produced a significant protection against NOD diabetes. However, thioredoxin has multiple actions in addition to being an antioxidant (Saitoh et al. 1998). Therefore to answer whether beta cell antioxidant protection is beneficial to prevent autoimmune diabetes, it is crucial to use more specific antioxidant proteins with more defined expression in the beta cell. Our transgenic models meet these requirements because the antioxidant proteins are expressed uniquely in beta cells, and the ROS scavenging effects of these antioxidants are efficient and specific. 


\section{Materials and Methods}

Maintenance of NOD mice and generation of transgenic NOD strain congenic for transgenes: NOD mice, purchased from Jackson Laboratory (Bar Harbor, Maine), were housed in individually ventilated microisolator cages at the Research Resource Center in University of Louisville. Strict cage changing and animal handling procedures were carried out to minimize viral infection. The standard mouse chow was Laboratory Rodent Diet 5001 (PMI Feeds, St. Louis, MO) containing 23\% protein and $4.5 \%$ fat. This diet was suitable for maintaining a high incidence of diabetes in NOD mice. Transgenic NOD mice with beta cell specific expression of catalase and MT were generated from our transgenic FVB mice using a speed congenic procedure described by Serreze, et al. (Serreze et al. 1996). In details, the MT and catalase transgenic FVB mice were backcrossed to NOD mice for up to 10 generations. Since the $5^{\text {th }}$ or $7^{\text {th }}$ generation, PCR based genotyping with the aid of microsatellite markers was performed to find a founder animal congenic for both transgene and all 19 NOD diabetic susceptible alleles. This animal was used as the progenitor for additional one or two backcrosses to NOD mouse for more homozygosity of NOD mouse genome. The produced transgenic and nontransgenic NOD offspring were intercrossed to generate animals which were used in this study.

PCR typing of NOD diabetes loci: The appropriate PCR primer pairs for polymorphic microsatellite markers of diabetic susceptible alleles of NOD mice were based on the report of Serreze et al (Serreze et al. 1996) and the Mouse Genome Database in Jackson Laboratory. Those primers were purchased from Research Genetics and listed in Table 5-1. Mouse tail DNA was extracted from transgenic NOD offspring and control FVB and NOD mice with Qiagen DNAeasy Kit (Qiagen, Valencia, CA) according to the manufacturer instructional manual. PCR reaction was performed in a $20 \mu \mathrm{l}$ volume containing $1.5 \mu \mathrm{l}$ sample DNA (about 20ng DNA), $200 \mu \mathrm{M}$ dNTP, $0.2 \mu \mathrm{M}$ of each primer, 0.45 unit of Platinum Taq DNA polymerase, $16 \mathrm{mM}\left(\mathrm{NH}_{4}\right)_{2} \mathrm{SO}_{4}, 67 \mathrm{mM}$ Tris-HCl, $\mathrm{pH} 8.8,0.01 \%$ TWEEN-20, and appropriate concentrations of $\mathrm{MgCl}_{2} . \mathrm{MgCl}_{2}$ concentrations were varied between 1 and $5 \mathrm{mM}$ based on different primer sets (Table 5-2). PCR reaction was carried out in an Eppendorf thermocycler equipped with a heated hood. The reaction contained a first polymerase activation step at $95^{\circ} \mathrm{C}$ for $2 \mathrm{~min}$, followed with 32 cycles of 
amplification, and ended with an extenstion step at $72^{\circ} \mathrm{C}$ for $7 \mathrm{~min}$. Each amplification cycle consisted of 45 seconds of denaturing at $94^{\circ} \mathrm{C}, 45$ seconds of annealing at $57^{\circ} \mathrm{C}$ and $1 \mathrm{~min}$ of extension at $72^{\circ} \mathrm{C}$. PCR products were fractionated on $6 \%$ NuSieve (FMC) agarose/TAE and stained with $0.5 \mu \mathrm{g} / \mathrm{ml}$ ethidium bromide. Control PCR reactions of inbred FVB and NOD mouse DNA were exerted every time and were fractioned in parallel to PCR products from other samples in order for a clear-cut discrimination of NOD diabetic allele from FVB normal allele. The PCR $\mathrm{Mg}^{2+}$ concentration and approximate size of PCR products from FVB and NOD strains for each set of microsatellite markers were summarized in Table 5-2.

H\&E staining and immunostaining for MT: Transgenic or control mouse pancreas was fixed in 10\%

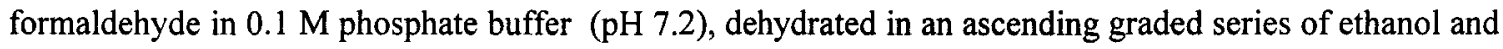
subsequently infiltrated with paraffin. Serial sections were cut at $5 \mu \mathrm{m}$, mounted on polylysine coated slides. Slides were stained with hematoxylin and eosin with a standard protocol. MT immune staining was carried out with a procedure as described in Materials and Methods of Part II, except that 3, 3'diaminobenzidine $(\mathrm{DAB})$ was used as the chromagen.

Cyclophosphamide administration and diabetes monitoring: Age and sex matched transgenic and nontransgenic NOD mice received two doses of intraperitoneal injection of $200 \mathrm{mg} / \mathrm{kg}$ body weight of cyclophosphamide. The first and second injections were two weeks apart. The mouse tail blood glucose level was monitored using a glucose meter (OneTouch Ultra, Life Scan) every other day after first injection of CYP. Mouse was considered diabetic after two consecutive readings of blood glucose over 200 $\mathrm{mg} / \mathrm{dl}$. The onset of diabetes was dated from the first of these two sequential measurements. Some mice were sacrificed after 8 days of CYP injection. Pancreas was removed for pancreatic insulin measurement, histology and immunohistochemistry study.

In vivo CYP administration to FVB mice: Two groups of transgenic HMT-1 and control FVB mice were used with age and sex ratio matched with above study in NOD mice. In one group, both transgenic and control FVB mice received two doses of intraperitoneal injection of $200 \mathrm{mg} / \mathrm{kg}$ body weight of cyclophosphamide, as did in the NOD mice. The first and second injection were two weeks apart. Another 
group of mice received a single intraperitoneal CYP injection at a dose of $380 \mathrm{mg} / \mathrm{kg}$ body weight. After CYP injection, mouse tail blood glucose level was monitored using a glucose meter (OneTouch Ultra, Life Scan) every other day. After 30 days for the group injected with low dose of CYP and 9 days for the group injected with high dose of CYP, mice were sacrificed and the pancreata were removed for measurement of preserved pancreatic insulin content.

Purification of mouse liver microsome (Devanesan et al. 1999). Normal FVB mouse was treated with cyclophosphamide $(300 \mathrm{mg} / \mathrm{kg}$ body weight, ip.) for 2 days for induction of liver microsome enzymes. The mouse liver was removed and homogenized in 3 volume of precooled Tris- $\mathrm{KCl}$ Buffer $(20 \mathrm{mM}$ Tris- $\mathrm{HCl}, \mathrm{pH} 7.4,0.15 \mathrm{M} \mathrm{KCl})$. The homogenate was centrifuged at $9,000 \times \mathrm{g}$ for $20 \mathrm{~min}$ at $4^{\circ} \mathrm{C}$. The supernatant was centrifuged at $105,000 \times \mathrm{g}$ for 1 hour at $4^{\circ} \mathrm{C}$ to obtain microsome pellet. After two washes with precooled Tris- $\mathrm{KCl}$ buffer, microsome pellet was resuspended in $1 \mathrm{ml}$ Tris- $\mathrm{KCl}$ buffer followed by a passage through $0.45 \mu \mathrm{M}$ filter. The filtered solution was determined by Pierce BCA protein assay kit for protein concentration and was then stored at $-70^{\circ} \mathrm{C}$ for next use.

In vitro CYP treatment on FVB islets: Assessment of CYP cytotoxicity to islets was carried out in a 96-well microplate with a modified procedure of Meyer et al. (Meyer et al. 1997). Ten overnight cultured HMT-1 transgenic or control FVB islets were incubated for $5 \mathrm{hr}$ in $200 \mu$ l culture medium (pH 7.4) containing $4 \mathrm{mM} \mathrm{NADP}+4 \mathrm{mM}$ glucose-6-phosphate, $2 \mathrm{mU} / \mathrm{ml}$ glucose-6-phosphate dehydrogenase, 75 $\mu \mathrm{g} / \mathrm{ml}$ liver microsome and appropriate concentrations of cyclophosphamide as indicated in the figure. After cyclophosphamide treatment, the culture medium was replaced with $200 \mu \mathrm{l}$ fresh culture medium and islets were incubated for another $16 \mathrm{hr}$. After incubation, both islet morphology and islet DNA content were analyzed.

Data analysis: The cumulative incidence of diabetes was calculated for each group and the significant difference was tested by Kaplan-Meier survival test using SPSS program (version 10.0). Two- 
way ANOVA and Dunnet's post hoc (2-tailed) test were used to test the difference between transgenic and control NOD mice before and after CYP treatment.

\section{$\underline{\text { Results }}$}

Production of transgenic NOD mice congenic for MT or catalase transgene: In this study, so far, lines on the NOD background with beta cell specific overexpression of antioxidants MT (designated MTNOD), and catalase (designated CatNOD) were derived from our FVB transgenic mice. The MnSOD transgene has been bred for several generations onto NOD mice but the MnSODNOD mice have not been achieved. For MTNOD mice, the MT transgenic FVB mice were backcrossed to NOD mice five times. On fifth generation, seven MT transgene positive offspring were selected for PCR genotyping of 19 diabetic susceptible alleles. The presence of the MT transgene in these progenitors, tentatively identified by their grayish coat color due to the co-injected transgene tyrosinase, was confirmed with PCR for the MT transgene. Two out of those seven mice were found homozygous for all 19 diabetic susceptible alleles (Figure 5-1). These two mice were used as founders to breed with NOD mice one more time. Currently the MT congenic NOD mice were maintained at the sixth generation by brother-sister breeding and these mice were used in this study.

In addition to PCR, MT transgene expression in pancreatic beta cells was also examined by immunohistochemistry. Figure 5-2 shows MT immunostaining and H\&E staining in pancreatic sections from MTNOD and NOD mice that were euglycemic and 150 days old. Compared to NOD control islet whose MT staining was negligible, MT staining was highly elevated in MTNOD islet. This expression of MT was specific to pancreatic islets in that almost no staining could be seen in the exocrine pancreas. This figure also showed that significant lymphocyte infiltration of pancreatic islets already occurred in both MTNOD and NOD prediabetic mice at 150 days of age (Figure 5-2, C and D), indicating the MT transgene did not prevent insulitis. Therefore these MTNOD mice should be useful to test MT induced resistance to Type 1 diabetes. 
For CatNOD mice, PCR analysis of homozygosity of all 19 diabetic susceptible alleles was started in the offspring at the $7^{\text {th }}$ generation of backcrossing of catalase FVB mice to NOD mice. But it was not until the $9^{\text {th }}$ generation that one mouse carrying the catalase transgene was found to be homozygous for all 19 diabetic susceptible alleles (data not shown). This mouse was backcrossed to NOD again. The offspring were intercrossed to maintain the line and produce transgenic and nontransgenic littermates used in this study. Similar to MTNOD, the CatNOD mice could be easily identified from their control littermates by coat color because of the second transgene, tyrosinase. However the presence of catalase transgene in that founder CatNOD mouse was also confirmed with PCR.

The effect of the MT transgene on NOD diabetes. Using these MTNOD and CatNOD mice we tested the potential effect of beta cell antioxidant transgenes on NOD diabetes. Fifteen MTNOD and 16 control NOD mice were injected with cyclophosphamide twice (at days 0 and 14) at a dose of $200 \mathrm{mg} / \mathrm{kg}$. After first injection diabetes onset and incidence were determined by the occurrence of hyperglycemia. To our great surprise, the MT transgene remarkably speeded up NOD diabetes (Figure 5-3) $\quad(\mathrm{P}<0.001$ by Kaplan-Meier survival analysis and Mantel-Cox Log-rank test), instead of delaying the onset of diabetes as we had hypothesized. In control NOD mice, diabetes generally did not occur until 22 days after the first injection of CYP. However in most MTNOD mice diabetes developed around ten days after the first injection of CYP.

This accelerated diabetes onset was confirmed by measurement of pancreatic insulin levels in control NOD and transgenic MTNOD mice before and 8 days after CYP injection. As shown in Figure 5-4, the pancreatic insulin content in MT transgenic and control NOD mice was similar before CYP injection. However, 8 days after CYP injection the pancreatic insulin level in MTNOD mice was only $25 \%$ of their original value before CYP injection; whereas the NOD control mice preserved almost $80 \%$ of their original insulin. This indicated that a many more beta cells were destroyed in MTNOD mice than in NOD mice, after CYP injection. Therefore these results suggest that MT overexpression in pancreatic beta cells makes NOD mice more susceptible to CYP accelerated autoimmune diabetes. 
This is a very puzzling result, completely opposite to our original hypothesis: that ROS scavengers would be helpful to prevent Type 1 diabetes. One possible explanation for this result is that the MT transgene sensitizes pancreatic beta cells to direct CYP toxicity. If true, this sensitizing effect should also be present in the FVB mice with beta cell overexpression of MT. Therefore we went back to MT transgenic FVB mice to test this possibility.

Protective effect of MT trangene against direct toxicity of CYP to pancreatic islets. To reveal whether the MT transgene increased direct CYP toxicity to beta cells, both in vivo and in vitro studies were performed. CYP is an anticancer drug with a lethal dose of $500 \mathrm{mg} / \mathrm{kg}$ in the mouse (Yan et al. 1991). In FVB mice CYP does not induce autoimmune diabetes, as it does on the NOD background, therefore in FVB mice any beta cell damage would be due to a direct effect of CYP on the beta cell. In the in vivo study, two groups of FVB mice containing or not containing the MT transgene were monitored for blood glucose levels after CYP injection. But neither one single high dose nor two low doses of CYP injection produced hyperglycemia in FVB and HMT-1 mice (Figure 5-5). The blood glucose level in HMT-1 mice was similar to (at the low dose of CYP) or probably a little lower (high dose of CYP) than that in FVB control mice. We also measured pancreatic insulin content in those mice one month (low dose of CYP) and 9 days (high dose of CYP) after CYP administration. We found that pancreatic insulin levels of both FVB and HMT-1 mice did not drop at all compared to the values from the animals without CYP injection (data not shown). This indicates that CYP does not have a direct toxic effect on beta cells when administrated in vivo.

However, activated CYP has indeed been shown to have a direct damaging effect on cultured cell lines. It causes DNA alkylation and apoptotic cell death (Marks \& Fox 1991; Sanderson \& Shield 1996). Therefore we also performed an in vitro study to test the potential effect of MT on isolated islets exposed to activated CYP. The activation of CYP was initiated by liver microsome extracts which contained $\mathrm{P}_{450}$ enzymes required for metabolism and activation of CYP. As shown in Figure 5-6, $0.5 \mathrm{mM} \mathrm{CYP}$ significantly caused morphological disruption in control FVB islets, but much less damage in HMT-1 islets. Islet DNA quantification also showed that the MT transgene significantly reduced islet DNA loss 
induced by activated CYP. MT induced DNA protection against CYP has also been shown in some cancer cell lines (Parsons et al. 1990; Wei et al. 1999). These data suggest that the MT transgene protects beta cells from direct toxicity of CYP, rather than sensitizing beta cells to CYP. The increased susceptibility to CYP induced NOD diabetes by the MT transgene is due to some other, still unidentified reason (s). But the similar result obtained with our other antioxidant, CatNOD line (see results below) suggests that antioxidant activity is involved in sensitization to NOD diabetes.

The effects of catalase transgene on NOD diabetes. Unlike the MT protein that scavenges many types of ROS, and also plays an important biological role in heavy metal homeostasis (Kumari et al. 1998), catalase is an antioxidant enzyme with relative simple action to dispose of $\mathrm{H}_{2} \mathrm{O}_{2}$. But interestingly, the catalase transgene behaved very similarly to the MT transgene in NOD mice. In Figure 5-7, eight CatNOD and 11 control NOD mice were treated with cyclophophamide with the same procedure as used on MTNOD mice. The data showed that the onset of NOD diabetes was significantly accelerated due to overexpression of beta cell catalase $(\mathrm{P}<0.05$ by Kaplan-Meier survival analysis and Mantel-Cox Log-rank test). Again around ten days after the first CYP injection diabetes occurred in most CatNOD mice, whereas most control NOD mice did not become diabetic until three weeks after the first injection of CYP. 


\section{Discussion}

In this part of our study, we produced two lines of transgenic NOD mice with elevated expression of MT and catalase specifically in pancreatic beta cells. Both MT and catalase transgenes made the NOD mice more susceptible to diabetes after CYP injection. The increased susceptibility was unlikely to be due to a direct damaging effect of the transgenes since the transgenic NOD mice had very similar pancreatic insulin level and islet morphology to control NOD mice. Also it was not due to a sensitization to direct CYP toxicity to beta cells by antioxidant transgenes because in the FVB strain, MT was shown to be protective against CYP. Our data suggest overexpression of antioxidant proteins in pancreatic beta cells increases the susceptibility to NOD diabetes.

This finding is at great variance with previous studies that ROS scavengers might be beneficial for beta cell survival during autoimmune diabetes. In NOD mice, increased formation of ROS and ROS derived oxidative damage has been found present in pancreatic beta cells themselves and islet-invading macrophages and lymphocytes (Horio et al. 1994; Suarez-Pinzon et al. 1997). Those ROS generally are considered to be detrimental factors causing beta cell death in autoimmune diabetes. Our MT and catalase transgenes can efficiently scavenge most types of ROS. But they actually exacerbate CYP accelerated NOD diabetes. Consequently our results suggest that the ROS produced in the beta cells are not merely detrimental factors leading to beta cell death in autoimmune NOD diabetes.

Extensive studies have been carried out showing the beneficial effects of antioxidant proteins. $\mathrm{MnSOD}, \mathrm{Cu} / \mathrm{Zn} \mathrm{SOD}$, catalase, and glutathione peroxidase, as well as some other antioxidant compounds have been shown to protect cultured cell lines or islets from exposure to ROS and cytokines (AzevedoMartins et al. 2003; Tiedge et al. 1997; Tiedge et al. 1998; Tiedge et al. 1999), and to reduce STZ and alloxan induced diabete (Welsh et al. 1995; Grankvist et al. 1981a; Uchigata et al. 1982). However, many of those studies were done in cell lines or on whole animals using systemic treatment of antioxidant compounds; thereby none of those treatments closely mimic human or NOD autoimmune diabetes. Several studies have demonstrated that injected antioxidant proteins or compounds protected both endogenous and 
transplanted islets in NOD mice (Bertera et al. 2003). But unlike our transgenes that were expressed only in pancreatic beta cells, the other procedure for antioxidant protein delivery effects all cells in the body, including the immune system. It is very possible that the protective effects observed with systemic administration of antioxidants may come from effects on the immune system. In the literature three transgenic animal models with beta cell specific expression of antioxidants have been described and tested against autoimmune diabetes. In one model pancreatic beta cell expression of thioredoxin (Hotta et al. 1998) prevented NOD diabetes; however thioredoxin is also an antiapoptitic protein, apart from being an antioxidant. In another model beta cell specific expression of extracellular SOD (Sandstrom et al. 2002) failed to prevent spontaneous NOD diabetes. Our study is the first study to find increased susceptibility to NOD diabetes due to antioxidant transgene expressions in pancreatic beta cells. Because the same results were obtained in two independent lines; catalase and MTNOD mice, the conclusion of increased susceptibility should be reliable.

However, the reason for the increased susceptibility to NOD diabetes by antioxidants has not yet been elucidated. In this study several possible artifacts have been ruled out: the prediabetic pancreatic insulin content and islet morphology in MTNOD and CatNOD mice are the same as that in control NOD mice, indicating no obvious damaging effect of the antioxidant transgenes; the antioxidant transgene does not sensitize to CYP toxicity directly in beta cells, in fact the MT transgene provides protection against CYP direct toxicity to cultured islets. Currently we know transgenic MTNOD beta cells die more rapidly than control NOD beta cells after cyclophosphamide injection. From these results we have a new hypothesis that permanent expression of antioxidants suppresses beta cell survival processes by scavenging ROS. This hypothesis and its background are discussed in greater details in the end of this dissertation, "Summary and Direction for Future Studies".

In addition to our hypothesis, several other important mechanisms may need also be considered to explain the increased diabetes susceptibility phenomenon. One possible mechanism is endoplasmic reticulum (ER) mediated stress (reviewed in (Harding \& Ron 2002; Oyadomari et al. 2002)). The pancreatic beta cells have a highly developed and active endoplasmic reticulum (ER) that leaves beta cells 
very susceptible to ER stress. Recent studies indicate that beta cell ER can sense and transduce apoptotic signals from various genetic and environmental stressors. ER stress-mediated beta cell apoptosis plays an important role in the development of diabetes. In our model, the overproduction of transgenic protein may impose a significant stress burden on beta cell ER and promote beta cell apoptosis. To test this possibility, we can measure the expression of some marker protein of ER stress in our transgenic NOD mice after CYP injection.

Another important question need to be considered is whether the increased susceptibility to CYP accelerated NOD diabetes can be extended to other types of autoimmune diabetes, such as spontaneous NOD diabetes and adoptive transfer NOD diabetes. CYP injected NOD mice supposedly develop autoimmune diabetes with similar pathology as in spontaneous NOD diabetes (Augstein et al. 1998). Beta cell destruction in each of these models is mediated by the autoimmune reaction. Therefore monitoring the diabetes incidence in spontaneous NOD diabetes models is necessary for a more complete understanding of the role of antioxidant transgenes in autoimmune Type 1 diabetes.

The third important factor is to know whether our antioxidant transgene is expressed ectopically, especially in the immune system. The insulin promoter is a strong promoter that has been shown in hundreds of transgenic mice to primarily target the pancreatic beta cell. Recent results indicate that the promoter is also active in brain cells that express insulin (Dandoy-Dron et al. 1995; Dandoy-Dron et al. 1991). However unlikely, it is possible that the MT transgene is also expressed in immune cells. This could alter their responsiveness to CYP. We can perform northern blots or immunohistochemistry to assure that antioxidant transgenes are negligibly expressed in tissues other than pancreatic beta cells. 
Table 5-1. Microsatellite linkage markes for diabetic susceptible alleles (Idds) homozygous for congenic NOD mice

\begin{tabular}{|c|c|c|c|}
\hline Idd locus & chromosome & genetic position (cM) & linkage marker \\
\hline $\operatorname{Idd} 1=H 2^{g /}$ & Chr17 & 19.5 & D17Mit34 \\
\hline Idd2 & Chr9 & 22 & D9Mit25 \\
\hline Idd3 & Chr3 & 19.2 & D3Mit21 \\
\hline Idd4 & Chr11 & 46 & D11Mit320 \\
\hline Idd5 & Chr1 & 40 & D1Mit18 \\
\hline Idd 6 & Chr6 & 73 & D6Mit339 \\
\hline Idd7 & Chr7 & 4 & D7Mit20 \\
\hline Idd8 & Chr14 & 3.5 & D14Mit222 \\
\hline Idd 9 & Chr4 & 82 & D4Mit357 \\
\hline Idd10 & Chr3 & 48.5 & D3Mit103 \\
\hline Idd11 & Chr4 & 64.6 & D4Mit148 \\
\hline Idd12 & Chr14 & 12 & D14Mit222 \\
\hline Idd13 & Chr2 & 71 & D2Mit395 \\
\hline Idd14 & Chr13 & syntenic & D13Mit61 \\
\hline Idd 15 & Chr5 & syntenic & D5Mit48 \\
\hline Idd16 & Chr17 & 18 & D17Mit50 \\
\hline Idd17 & Chr3 & 39 & D3Mit230 \\
\hline Idd18 & Chr3 & 53.3 & D3Mit103 \\
\hline Idd19 & Chr6 & 60.5 & D6Mit25 \\
\hline
\end{tabular}

Adapted from Serreze DV, et al. (1996) J. Exp. Med. 184:2049-2053, and modified based on Mouse Genome Database from Jackson Laboratory (Serreze et al. 1996). 
Table 5-2. $\mathrm{Mg}^{2+}$ concentration for PCR reaction and approximate PCR product size for each set of microsatellite markers from NOD and FVB strain

\begin{tabular}{|c|c|c|c|c|}
\hline Idd locus & linkage marker & $\begin{array}{c}\text { NOD size } \\
\text { (bp) }\end{array}$ & $\begin{array}{l}\text { Approximate } \\
\text { FVB size (bp) }\end{array}$ & $\begin{array}{l}\mathrm{Mg}^{2+} \text { concentration } \\
(\mathrm{mM}) \text { for } \mathrm{PCR} \text { reaction }\end{array}$ \\
\hline Idd1 & D17Mit34 & 126 & 146 & 2 \\
\hline Idd2 & D9Mit25 & 136 & 128 & 5.8 \\
\hline Idd3 & D3Mit21 & 218 & 230 & 3.2 \\
\hline Idd4 & D11Mit320 & 124 & 128 & 3.5 \\
\hline Idd5 & D1Mit18 & 170 & 160 & 3.2 \\
\hline Idd6 & D6Mit339 & 110 & 125 & 3 \\
\hline Idd7 & D7Mit20 & 94 & 102 & 4 \\
\hline Idd8/12 & D14Mit222 & 136 & 120 & 5 \\
\hline Idd9 & D4Mit357 & 112 & 105 & 4.2 \\
\hline Idd10/18 & D3Mit103 & 130 & 142 & 3 \\
\hline Idd11 & D4Mit148 & 128 & 145 & 3.4 \\
\hline Idd13 & D2Mit395 & 120 & 162 & 3 \\
\hline Idd14 & D13Mit61 & 180 & 170 & 2.8 \\
\hline Idd15 & D5Mit48 & 150 & 200 & 2.5 \\
\hline Idd16 & D17Mit50 & 122 & 114 & 2.2 \\
\hline Idd17 & D3Mit230 & 146 & 148 & 3.5 \\
\hline Idd19 & D6Mit25 & 122 & 127 & 2.5 \\
\hline
\end{tabular}



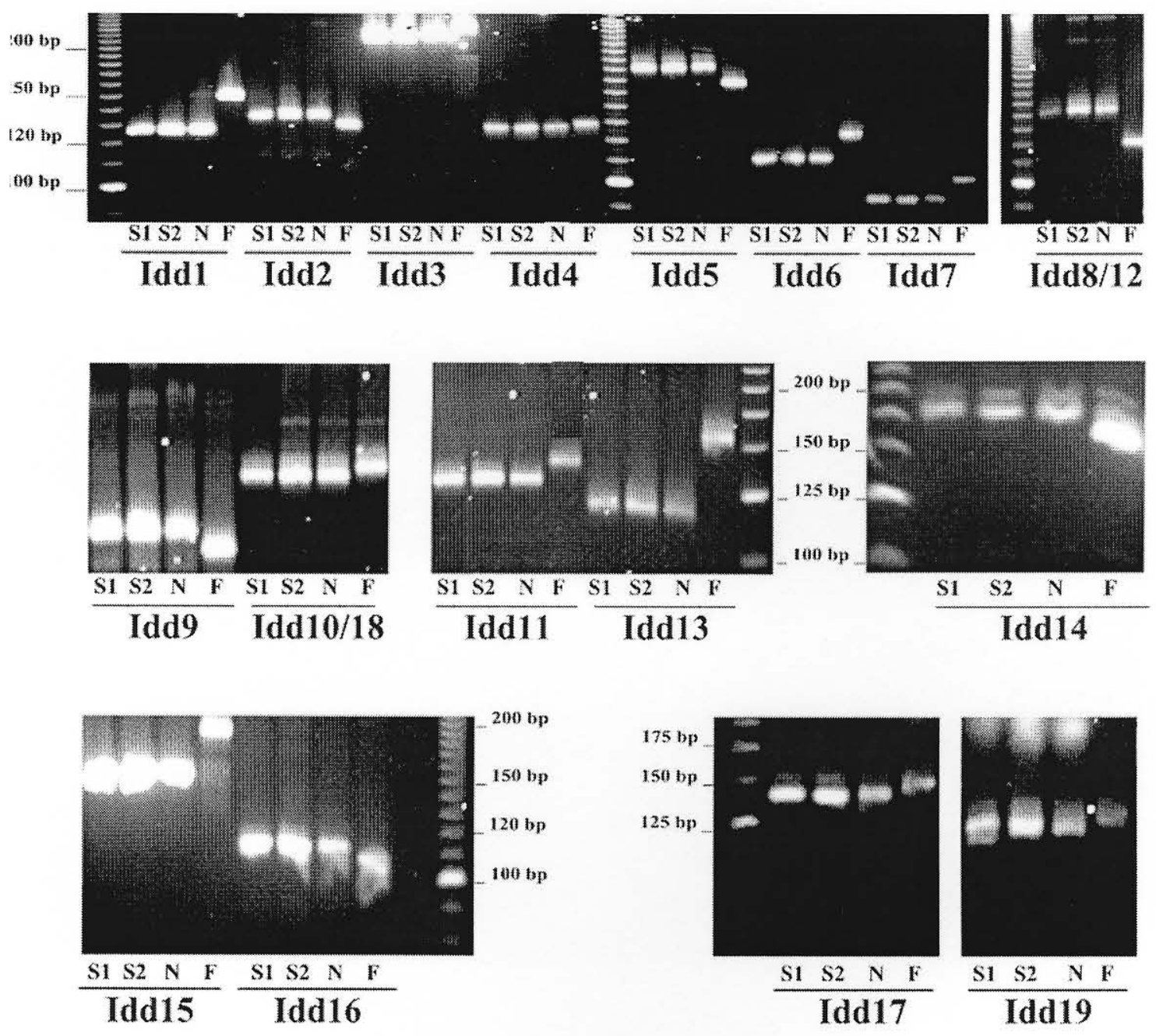

Figure 5-1. PCR-based genotyping of diabetic susceptible alleles in congenic NOD mice with $\beta$-cell specific expression of MT transgene. Homozygosity of 19 NOD diabetes loci (Idd) in the congenic NOD mice was determined by PCR using polymorphic microsatellite markers linked with these Idds. This figure is a representative gel graph showing 19 Idds in two MT congenic progenitors (S1, S2), inbred NOD (N), and FVB (F) mice. 


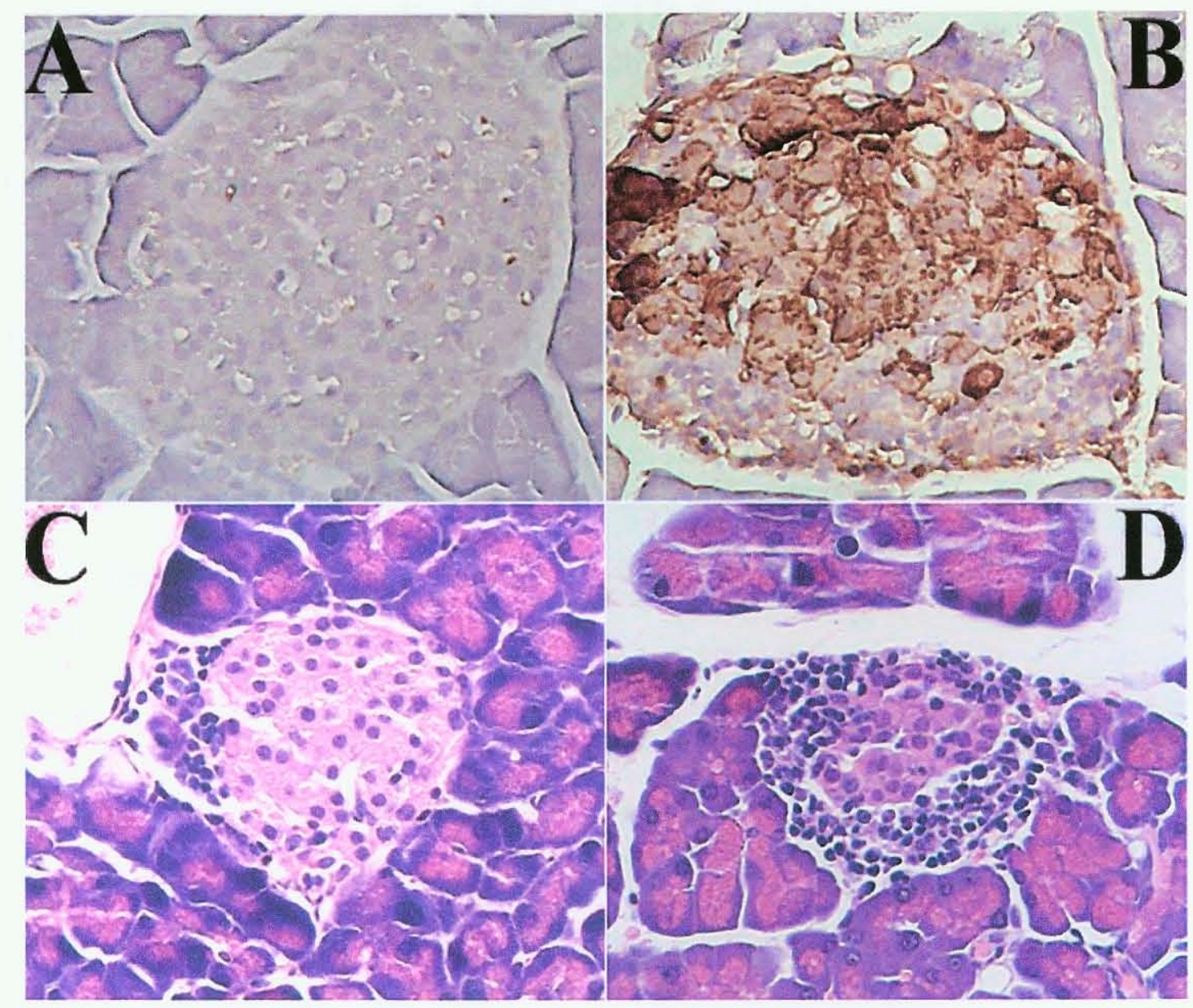

Figure 5-2. MT staining and insulitis in NOD and MTNOD congenic mice at the age of 150 days. Pancreatic sections in A (NOD) and B (MTNOD) were stained with a monoclonal anti-horse MT primary antibody. Magnification $\times 400$. MT transgene specifically expressed in MTNOD islets at a high level, whereas alnost no expression was found in control NOD islets and acinar tissues of both MT and control mice. Sections in C (NOD) and D (MTNOD) were stained with hematoxylin and cosin. Magnification $\times$ 200. MTNOD mice developed similar insulitis as control NOD mice did. 


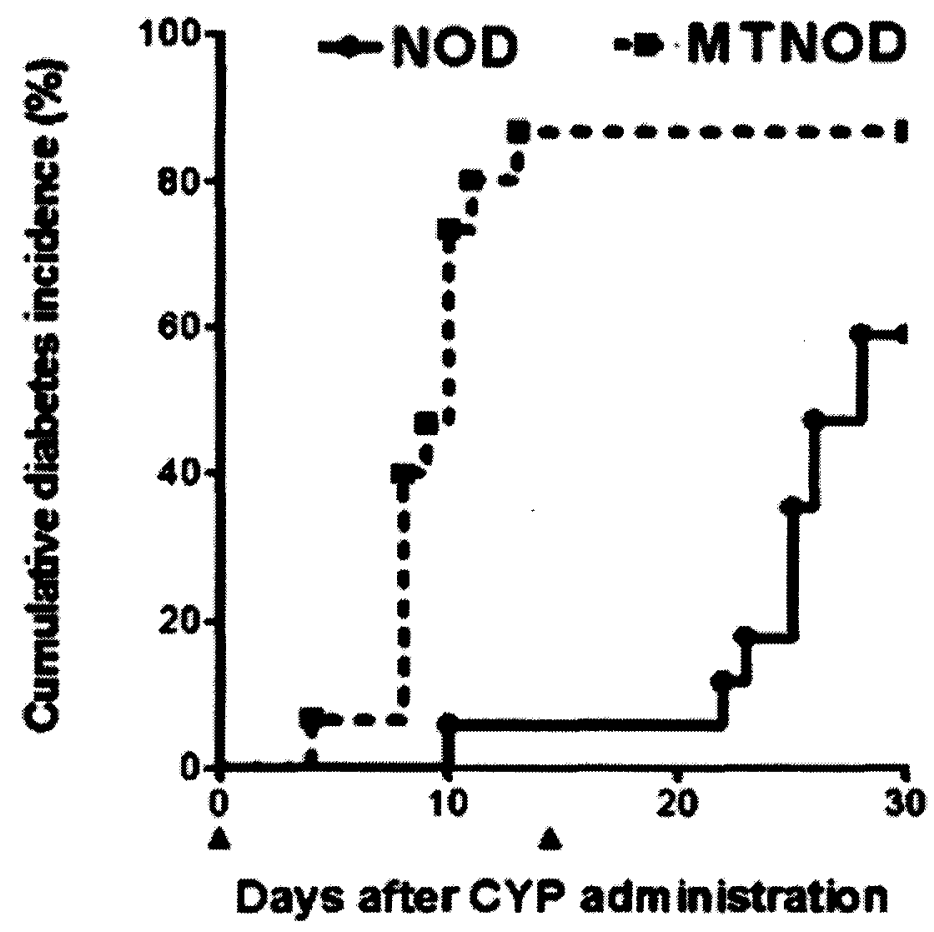

Figure 5-3. Cumulative diabetes incidence in MT transgenic and nontransgenic NOD mice injected with CYP. Groups of 16 nontransgenic NOD mice and 15 MT transgenic NOD mice, at the age of 9-10 weeks and sex-matched, were injected with CYP $(200 \mathrm{mg} / \mathrm{kg}$ body weight via ip.) at days 0 and 14 ( $\mathbf{\Delta})$. Tail blood glucose was monitored every other day. Onset of diabetes was defined when the first of two consecutive measurements of nonfasting blood glucose levels $>200 \mathrm{mg} / \mathrm{dl}$ was reached. Kaplan-Meier survival analysis and Mantel-Cox Log-rank test revealed that MT significantly hastened NOD diabetes onset $(\mathrm{P}<0.001)$. 


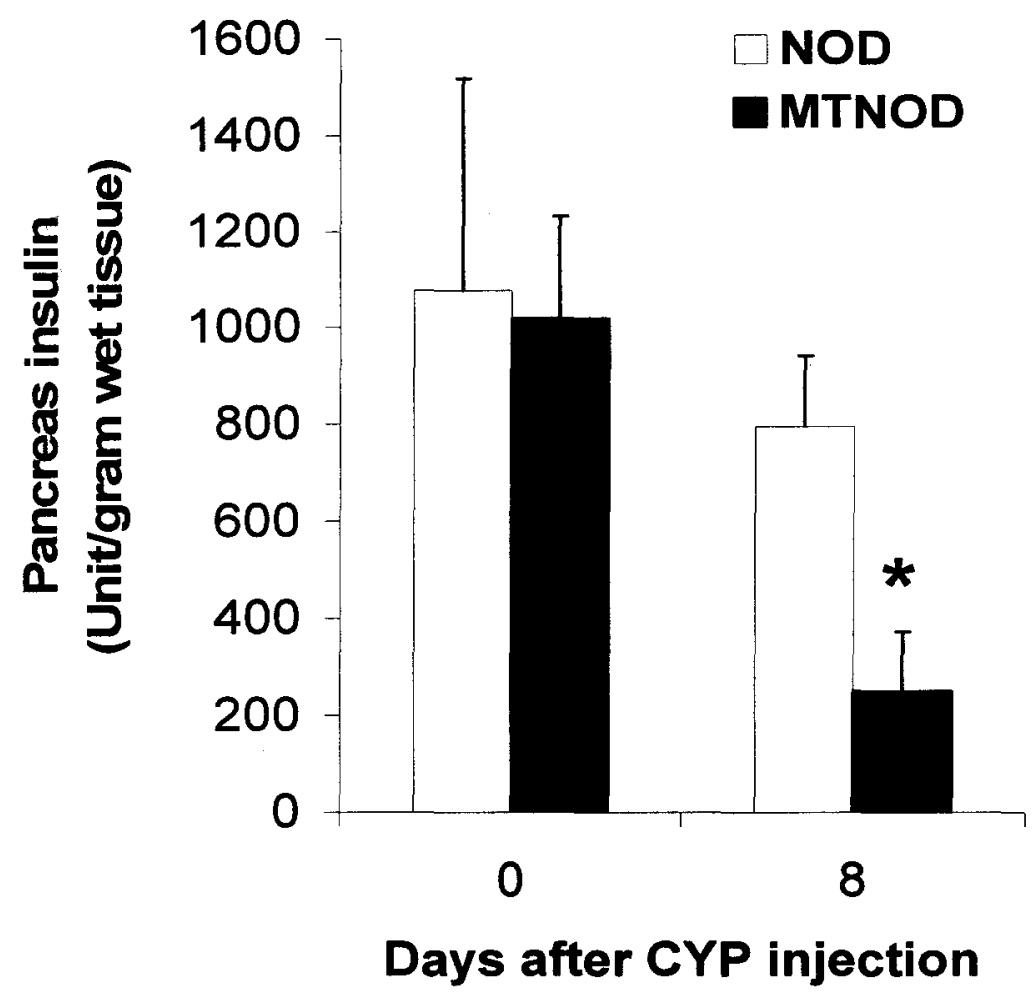

Figure 5-4. Preserved pancreatic insulin levels in MT transgenic and nontransgenic NOD mice 8 days after CYP administration. Pancreatic insulin levels were measured by an insulin antibody coated tube RIA kit as described in Materials and Methods. Data are expressed as mean \pm SE, taken from 3 or 4 mice in each group. * $\mathrm{P}<0.05$ in NOD vs. MTNOD 8 days after CYP injection by one-way ANOVA. Vertical bars show standard error of the mean. 


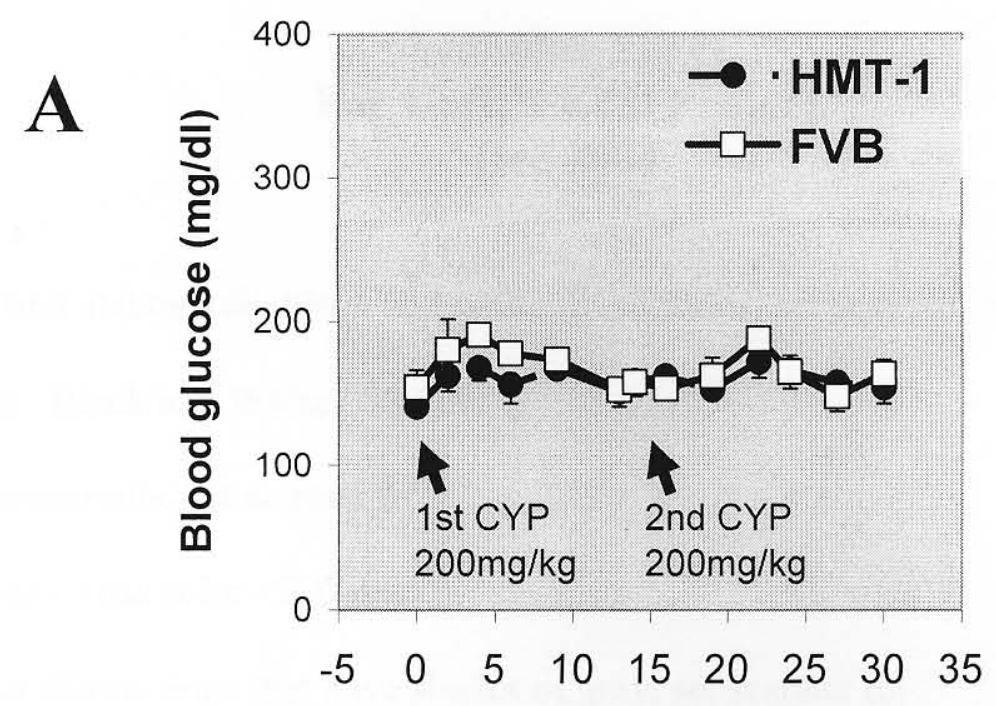

\section{Days after CYP administration}

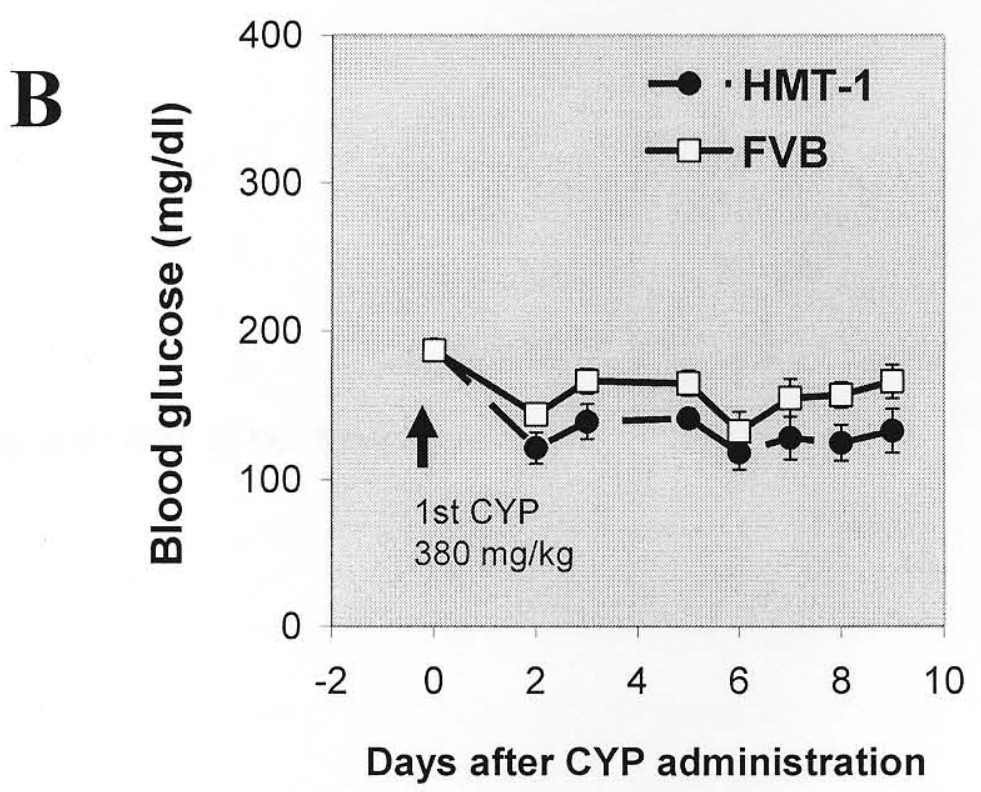

Figure 5-5. No incidence of diabetes in FVB control and HMT-1 mice after CYP administration. Both MT transgenic (HMT-1) FVB mice and control FVB mice were injected with two low doses (A) or a single high dose (B) of CYP as indicated. Diabetes onset was monitored based on occurrence of hyperglycemia as described in Materials and Methods. CYP did not cause diabetes in the FVB strain. MT transgene did not sensitize FVB mice to diabetes. 

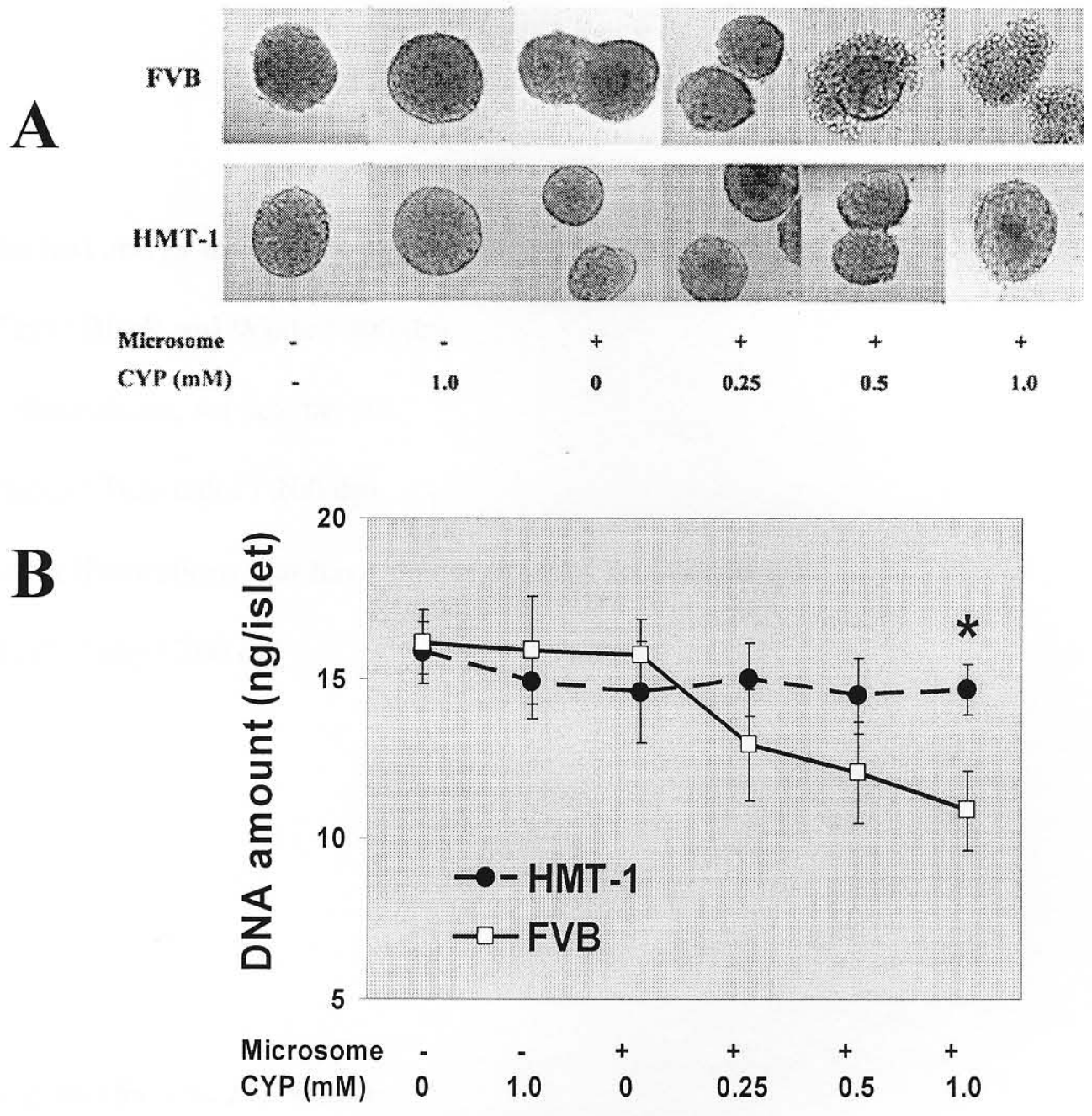

Figure 5-6. Protective effect of MT transgene against CYP mediated cytotoxicity to cultured islets from the FVB strain. Both transgenic (HMT-1) and nontransgenic (FVB) islets from FVB background were cultured in a medium containing indicated concentrations of CYP and mouse liver microsome $\left(\mathrm{P}_{450}\right)$ extract that catalyzes CYP to active and toxic form. After treatment for 5 hours, islet culture medium was changed with fresh medium and islets were incubated for 16 hours. Both islet morphology (A) and islet DNA content (B) were examined. MT transgene reduced CYP induced FVB islet morphological disruption and DNA loss. Results came from 4 independent experiments. ${ }^{*} \mathrm{P}<0.05$ HMT-1 vs. FVB at the corresponding concentration of CYP by one-way ANOVA. Vertical bars indicate standard error of the mean. 


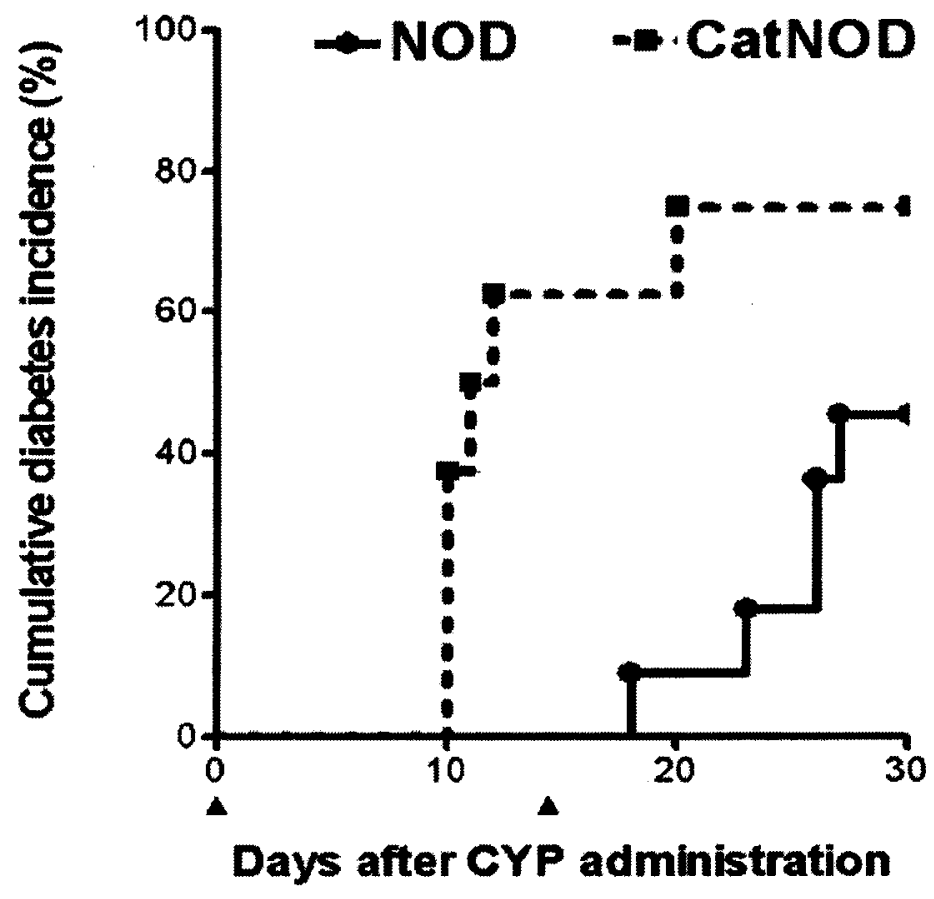

Figure 5-7. Acceleration of diabetes by $\beta$-cell specific catalase transgene in CYP injected NOD mice. Groups of 11 nontransgenic NOD mice and 8 catalase transgenic NOD mice (CatNOD), at the age of 5-6 weeks and sex-matched, were injected with CYP $(200 \mathrm{mg} / \mathrm{kg}$ body weight via ip. ) at days 0 and 14 (A). Diabetes onset was monitored and defined as described in Materials and Methods. Kaplan-Meier survival analysis and Mantel-Cox Log-rank test revealed that catalase transgene significantly speeded up NOD diabetes onset $(\mathrm{P}<0.05)$. 


\section{CHATER VI}

\section{THE ROLE OF ANTIOXIDANT TRANSGENES}

\section{IN AGOUTI OBESE DIABETIC MICE AND NORMAL C57BL/KsJ MICE}

\section{$\underline{\text { Introduction }}$}

Several lines of emerging evidence have demonstrated that ROS contribute to pancreatic beta cell dysfunction and beta cell degeneration in Type 2 diabetes. Chronic exposure to hyperglycemia and hyperlipidemia probably results in excessive ROS production in pancreatic beta cells (Tanaka et al. 2002; Listenberger et al. 2001). In both patients (Sakuraba et al. 2002) and animal models of Type 2 diabetes (Ihara et al. 1999), beta cell oxidative stress markers due to the oxidation of cellular DNA, lipid and protein are significantly increased. Some antioxidant compounds, such as $\mathrm{N}$-acetylcysteine (NAC), aminoguanidine and $\alpha$-tocopheral have been shown to be beneficial for diabetes treatment. These agents not only preserved insulin secretory function and improved cell viability in insulin secreting cell lines exposure to high level of glucose (Tanaka et al. 1999b), but effectively protected from Type 2 diabetes in animal models (Ihara et al. 2000; Kaneto et al. 1999; Laybutt et al. 2002) as well. A recent study (Tanaka et al. 2002) reported that adenoviral overexpression of glutathione peroxidase provided protection against high glucose toxicity by preserving islet insulin mRNA, islet insulin content and secretion in rat islets treated with high level of ribose. Therefore, a common hypothesis has been proposed that antioxidant therapy is beneficial to prevent beta cell dysfunction and degeneration in Type 2 diabetes. However, this concept has not been tested in any in vivo study using animal models of Type 2 diabetes combined with beta cell specific expression of antioxidants. For this, we produced lines of antioxidant transgenic mouse crossed with models of Type 2 diabetes. These antioxidant proteins are specifically expressed in beta cells and thereby their potential site of protection is confined to the beta cells. 
Agouti mice and $\mathrm{db} / \mathrm{db}$ mice are two animal models which we originally planned to use in this study to test overexpression of beta cell antioxidants. The Agouti mouse (Michaud et al. 1994), which we obtained from the Jackson laboratory, is maintained on the inbred $\mathrm{KK}$ background. This mouse is heterozygous for a dominant mutated gene called lethal yellow (Ay) at the Agouti locus (a) of mouse chromosome 2. Mouse homozygous for Ay gene cannot survive before implantation or shortly thereafter. But mice heterozygous for the Ay gene are obese and develop Type 2 diabetes (Bultman et al. 1992; Michaud et al. 1997). The normal murine Agouti gene is transiently expressed only in neonatal skin, where it causes melanocytes to produce normal pigment (Black fur) (Siracusa 1994). However the mutated form of the Agouti gene causes a yellow coat color and cause ectopic expression of the Agouti protein in many other organs. This ectopic expression of the Ay gene makes the mouse develop a form of Type 2 diabeteslike syndrome, with characteristics of human Type 2 diabetes such as obesity, insulin resistance, hyperinsulinemia, pancreatic islet hypertrophy and hyperplasia (Klebig et al. 1995).

The molecular mechanism by which the agouti protein causes diabetic syndrome is unclear. Some studies have demonstrated that recombinant agouti protein can coordinately regulate adipocyte lipogenesis and lipolysis; thereby promoting lipid storage and obesity (Jones et al. 1996; Xue et al. 1998). The adipocytes from Agouti mice are less sensitive to insulin as assessed by insulin stimulated glucose uptake and oxidation, indicating that the agouti protein may also cause insulin resistance. Several recent studies reported that agouti protein regulated cellular $\mathrm{Ca}^{2+}$ signaling in various tissues, including adipocytes (Xue et al. 1998) and pancreatic islets (Xue et al. 1999). It has been shown that agouti protein is expressed in human pancreas, and stimulates both $\mathrm{Ca}^{2+}$ signaling and insulin release from human pancreatic islets. This is a possible reason why hyperinsulinemia occurs in Type 2 diabetes. Agouti protein has also been shown to stimulate islet amyloid polypeptide release in human pancreatic islets via a $\mathrm{Ca}^{2+}$ dependent mechanism (Xue \& Zemel 2001). The islet amyloid polypeptide is the major component of islet amyloid deposits that are often found in islets of Type 2 diabetic patients. Excessive amyloid deposits in pancreatic islets is thought to diminish beta cell mass and cause beta cell dysfunction and degeneration in the later stage of Type 2 diabetes (Leffert et al. 1989; Lorenzo et al. 1994). Compared to their wild-type counterparts, the 
Agouti mouse has elevated levels of plasma amyloid polypeptide (Warbritton et al. 1994; Verchere et al. 1996).

As indicated above the Agouti mouse we selected in this study was maintained on the KK background. The KK strain has inherent glucose intolerance and insulin resistance but it has normal blood glucose and is not obese (Reddi \& Camerini-Davalos 1988). But with the addition of the mutated Ay gene, KK mice become overtly diabetic and obese. By eight weeks of age, the Agouti KK mouse develops obesity, hyperinsulinemia and hyperglycemia. Male mice are more sensitive than female mice (Klebig et al. 1995). Since our beta cell antioxidant transgenes are maintained on FVB background, the transgenic FVB mice were cross-bred with Agouti KK mice to produce F1 mice. We found that those F1 mice, carrying the Agouti gene (identified by a yellow coat pigment) spontaneously became obese and diabetic. Therefore in this study we used the F1 Agouti mouse as a model of obesity-associated Type 2 diabetes for evaluating the potential beneficial effect of beta cell antioxidant transgenes.

Another model of Type 2 diabetes we originally planned to use is the $\mathrm{db} / \mathrm{db}$ mouse. This type of mouse is a strain of inbred background called C57BL/KsJ containing a homozygous mutated leptin receptor gene $\left(\right.$ Lepr $\left.^{\mathrm{db}}\right)$, usually designated as $\mathrm{db} / \mathrm{db}$ (Berglund et al. 1978; Leiter et al. 1981). The leptin ligand/leptin receptor genes play important roles in control of body weight (Igel et al. 1997; Tartaglia 1997). Without $\mathrm{a} \mathrm{db} / \mathrm{db}$ homozygous mutation, the strain of C57BL/KsJ mouse is lean and euglycemic. But if it carries the $\mathrm{db} / \mathrm{db}$ homozygous mutation, this background of $\mathrm{C} 57 \mathrm{BL} / \mathrm{KsJ}$ becomes obese and diabetic. Introduction of the same $\mathrm{db} / \mathrm{db}$ mutation onto other genetic backgrounds, such as FVB or C57BL/6J only results in obesity but not diabetes (Chua S Jr et al. 2002). The reason that the C57BL/KsJ background is required for $\mathrm{db} / \mathrm{db}$ mediated Type 2 diabetes is unclear. In $\mathrm{db} / \mathrm{db}$ mice with a C57BL/KsJ background, obesity starts around 3 to 4 weeks of age, hyperinsulinemia occurs two weeks after birth and hyperglycemia develops at 4 to 8 weeks of age. Thus in this study we first placed the antioxidant transgenes on the C57BL/KsJ background by backcrossing our transgenic FVB mice to $\mathrm{C} 57 \mathrm{BL} / \mathrm{KsJ}$ mice up to six times. We next intended to introduce the $\mathrm{db} / \mathrm{db}$ mutation by further backcrossing with Lepr $^{\mathrm{db}}$ gene heterzygous $(\mathrm{db} /+) \mathrm{C} 57 \mathrm{BL} / \mathrm{KsJ}$ mice. Since C57BL/KsJ mice are normally euglycemic we expected 
that until we added the $\mathrm{db} / \mathrm{db}$ homozygous mutation that our animals with antioxidant transgenes would be euglycemic. However, we found that C57BL/KsJ mouse with the MT beta cell transgene developed severe diabetes even before the $\mathrm{db} / \mathrm{db}$ mutation was introduced. . 


\section{Materials and Methods}

Generation of transgenic Agouti F1 mice and C57BL/KsJ mice. Agouti KK mice and C57BL/KsJ inbred mice were purchased from Jackson Laboratory and housed in ventilated microisolator cages at the Research Resource Center in University of Louisville. The mice were fed with standard mouse chow with free access to tap water.

The transgenic Agouti mice were F1 litters produced by the following breeding procedures. MT transgenic F1 Agouti mice were produced by cross-breeding between HMT-1 transgenic FVB mice and Agouti $\mathrm{KK}$ inbred mice. MnSOD, catalase and MnSOD plus catalase transgenic F1 Agouti mice were produced by cross-breeding between inbred FVB mice containing both MnSOD and catalase transgenes to Agouti KK inbred mice. Among the produced F1 offspring, half of them were theoretically Ay gene negative mice with a black coat color, and another half were Ay gene positive mice with a yellow coat color. The presence of antioxidant transgenes was identified by PCR.

The MT transgenic C57BL/KsJ mice were produced by continuous backcrossing of HMT-1 transgenic FVB mice to C57BL/KsJ inbred mice up for six times. At each generation, the MT transgenic offspring were determined by PCR.

Monitoring of diabetes and obesity in Agouti F1 mice: The non-fasting tail blood glucose level was periodically measured with a glucose meter (Ultrasensitive, LifeScan) in the Agouti F1 mice and control mice (non Agouti, non transgenic) shortly after they were weaned. The body weight was also recorded at the same time. The diabetes onset was defined as the first day when two consecutive measurements of blood glucose level were higher than $200 \mathrm{mg} / \mathrm{dl}$. Some Agouti F1 mice were sacrificed at 3 months and 7 months of age and blood samples were collected for measurement of glycohemoglobin.

Measurement of whole blood glycohemoglobin. The assay of blood glycohemoglobin measurement was performed by a Glyc-Afin GHb kit from PerkinElmer Life Sciences (Norton, OH). This is an affinity- 
based chromatography assay. Briefly, the mouse blood was collected into a collection microtube coated with heparin. $50 \mu \mathrm{l}$ of the blood sample was mixed with $400 \mu \mathrm{l}$ sample preparation reagent which was supplied in the kit. After standing for $10 \mathrm{~min}$ with occasional shaking, $50 \mu \mathrm{l}$ of this mixture was added onto the top disk of the affinity column that was prewashed with preparation solution. The sample mixture was allowed to flow into the column freely and thereafter the column was washed with $100 \mu$ First Fraction Elution Agent supplied in the kit. Then the elute containing nonglycohemoglobins was collected by adding $3 \mathrm{ml}$ of First Fraction Elution Agent to the column followed by a collection of glycohemoglobin elute washed by $2 \mathrm{ml}$ of Second Fraction Elution Agent. The nonglycohemoglobin fraction was diluted with addition of $17 \mathrm{ml}$ of distilled water. The optical density of these glycohemoglobin and diluted nonglycohemoglobin elutes was measured at the wavelength of $405 \mathrm{~nm}$. The blood glycohemoglobin content was calculated as:

$$
\% \mathrm{GHb}=100 * \mathrm{G} /(\mathrm{G}+10 \mathrm{~N})
$$

where $\mathrm{G}=$ absorbance of the $\mathrm{GHb}$ fraction, and $\mathrm{N}=$ absorbance of the nonglycohemoglobin fraction. This formula was provided by the kit.

Diabetes in MT transgenic C57BL/KsJ mice: At each generation the offspring were carefully observed for diabetic symptoms (polyphagic, polydipsic, and polyuric). At certain age or whenever the mice were found with some diabetic symptoms, the tail blood glucose levels were checked with a glucose meter (Ultrasensitive, LifeScan). Some mice were sacrificed at different ages and pancreata were removed for standard histological H\&E staining. Both transgenic and nontransgenic mouse pancreatic insulin levels at different ages were measured with a radioactive immunoassay as described in Materials and Methods of Part II. 


\section{$\underline{\text { Results }}$}

Obesity and diabetes in Agouti F1 mice. We produced F1 mice by cross-breeding between our heterozygous transgenic FVB mice and heterozygous Agouti KK mice. This breeding procedure generated littermates carrying $50 \%$ of genes from FVB and $50 \%$ from $\mathrm{KK}$. Therefore these $\mathrm{F} 1$ mice were genetically identical except for the Ay gene and our antioxidant transgenes. To evaluate the effect of MnSOD and/or catalase antioxidant transgenes, we produced F1 mice by cross-breeding double transgenic FVB mice (containing both MnSOD and catalase) and Agouti KK inbred mice. These litters had eight possible genotypes. Each of them theoretically should occupy one-eighth of the population. Figure 6-1 showed the body weight and blood glucose levels in five types of F1 male mice. They are control mice (nontransgenic

and non-Agouti), nontransgenic Agouti, MnSOD Agouti, catalase Agouti, and MnSOD+catalase Agouti mice. Compared with the control mice, all four types of Agouti F1 mice, no matter what kind of transgene they carried, developed similar obesity as they aged (Figure 6-1A). Those Agouti F1 mice had a blood glucose level close to $200 \mathrm{mg} / \mathrm{dl}$ at the age of one month which was already significant higher than the control mice. At the age of 2 to 3 months, the blood glucose for all types of Agouti F1 mice climbed to a level higher than $300 \mathrm{mg} / \mathrm{dl}$, and then maintained there until the end of our observation. There was a nonsignificant trend for catalase and catalase plus MnSOD transgenic F1 Agouti mice to have greater hyperglycemia than either Agouti F1 or Agouti MnSOD F1 mice. Out data suggest that MnSOD and catalase transgenes, either alone or in combination, do not significantly affect the progression of diabetes in Agouti F1 obese mice.

We also measured whole blood glycated hemoglobin concentrations in those Agouti F1 mice at the age of 3 months and 7 months. Glycated hemoglobin refers to total glycosylated hemoglobin present in erythrocytes. It reflects the level of glucose in the blood stream over the previous four to eight weeks. Compared to blood glucose or urine glucose measurements whose values can vary over the course of a day, glycated hemoglobin level are more stable. In normal euglycemic mice the glycated hemoglobin value is reported to be under $6 \%$. In our study, nontransgenic Agouti F1 mice had a glycated hemoglobin level of $5.6 \%$ at 3 months of age and elevated to $9 \%$ at 7 months of age, indicating a gradual progression of Type 2 
diabetes. However, the antioxidant transgene, either alone (MnSOD or catalase) or combined together, had no therapeutic value to reduce glycohemoglobin content (Figure 6-2).

To test the role of the MT transgene in Agouti Type 2 diabetes, the HMT-1 transgenic FVB mice were bred with Agouti KK inbred mice. Four types of F1 littermates were produced which were MT Agouti, nontransgenic Agouti, MT non-Agouti, and nontransgenic non-Agouti. All Agouti mice (MT transgenic or nontransgenic) developed obesity but non-Agouti mice (MT transgenic or nontransgenic) were lean (data not shown). We followed the blood glucose in male F1 mice over 4 months. As shown in Figure 6-3, the control mice with no Agouti gene and no MT transgene maintained euglycemia. But with the Agouti gene the mice gradually became hyperglycemic. There was no statistical difference between MT transgenic Agouti mice and nontransgenic Agouti mice although the MT transgenic mice always had a little higher glucose level. Therefore the MT transgene did not prevent Type 2 diabetes in Agouti mice.

The effect of the MT transgene in C57BL/KsJ mice. A C57BL/KsJ mouse is a strain with normal blood glucose throughout life although this strain is unusually sensitive to many diabetogens, such as STZ, alloxan and certain viruses (Berglund et al. 1988; Blokh et al. 1988; Leiter et al. 1987a; Leiter et al. 1983). In our study we placed the MT transgene on C57BL/KsJ background by backcrossing HMT-1 transgenic FVB mice to C57BL/KsJ inbred mice. Since we had not introduced the mutated leptin receptor gene $\mathrm{db}$, the offspring were not expected to become obese or diabetic. However, even in the F1 generation we unexpectedly found that MT produced severe diabetes (blood glucose $>600 \mathrm{mg} / \mathrm{dl}$ ) in some offspring. The severity of diabetes seemed to be age-, sex- and backcross generation dependent (Table 6-1). Now at the $6^{\text {th }}$ generation, $100 \%$ of male MT transgenic mice become diabetic at 8 to 10 weeks, and about $40 \%$ of female MT mice become diabetic at the age of 20 weeks. Neither male nor female mice without the MT transgene develop diabetes (Figure 6-4). Morphological observation by H\&E staining revealed significant atrophy in pancreatic islets and degeneration of islet cells (Figure 6-5). 


\section{Discussion}

In this part of study, we developed several lines of F1 mice heterozygous for the Agouti gene (Ay) by outbreeding between antioxidant transgenic FVB mice and Agouti/KK mice. The F1 nontransgenic Agouti mice spontaneously developed obesity and hyperglycemia by 6 weeks of age. However the progression of obesity and diabetes in this strain of mice was not altered by the beta cell antioxidant transgenes; including MT, catalase, MnSOD and MnSOD plus catalase. These data indicate that antioxidant therapy is not likely to be beneficial for beta cell function and survival in the Agouti Type 2 diabetic model.

This is the first study to test the effect of antioxidant transgenes against Type 2 diabetes in vivo. The F1 mice obtained by crossbreeding between FVB and Agouti KK mice developed obesity and diabetes. This makes the Agouti model extremely convenient for testing transgenes against Type 2 diabetes. Random serum insulin measurements (Data not shown) indicated that the F1 mice were initially hyperinsulinemic. Pancreatic histology at an early age showed that the Agouti F1 mice had very large islets (Data not shown). Both the hyperinsulinemia and large islets are typical of beta cell compensation that generally precedes Type 2 diabetes.

However the results were disappointing in that we did not observe any protective effect of our antioxidant transgenes. These antioxidants were unable to reduce the hyperglycemia in F1 Agouti mice. This indicates the failure of pancreatic beta cells to adequately compensate for insulin resistance is not likely to be due to oxidative stress.

The finding that MT induced diabetes on the C57BL/KsJ background was extremely surprising. $\mathrm{C} 57 \mathrm{BL} / \mathrm{KsJ}$ mice are very sensitive to diabetes from many sources. This appears to be due to the ease with which their beta cells die. Most studies on beta cells of C57BL/KsJ mice were done before the appreciation of the apoptosis process so it has not been determined whether cell death is due to apoptosis or necrosis. Overexpression of MT must trigger this death process and the mechanism remains to be 
determined. It may be related to the increased rate of diabetes onset we found in CYP treated MTNOD mice, described in Part V. 

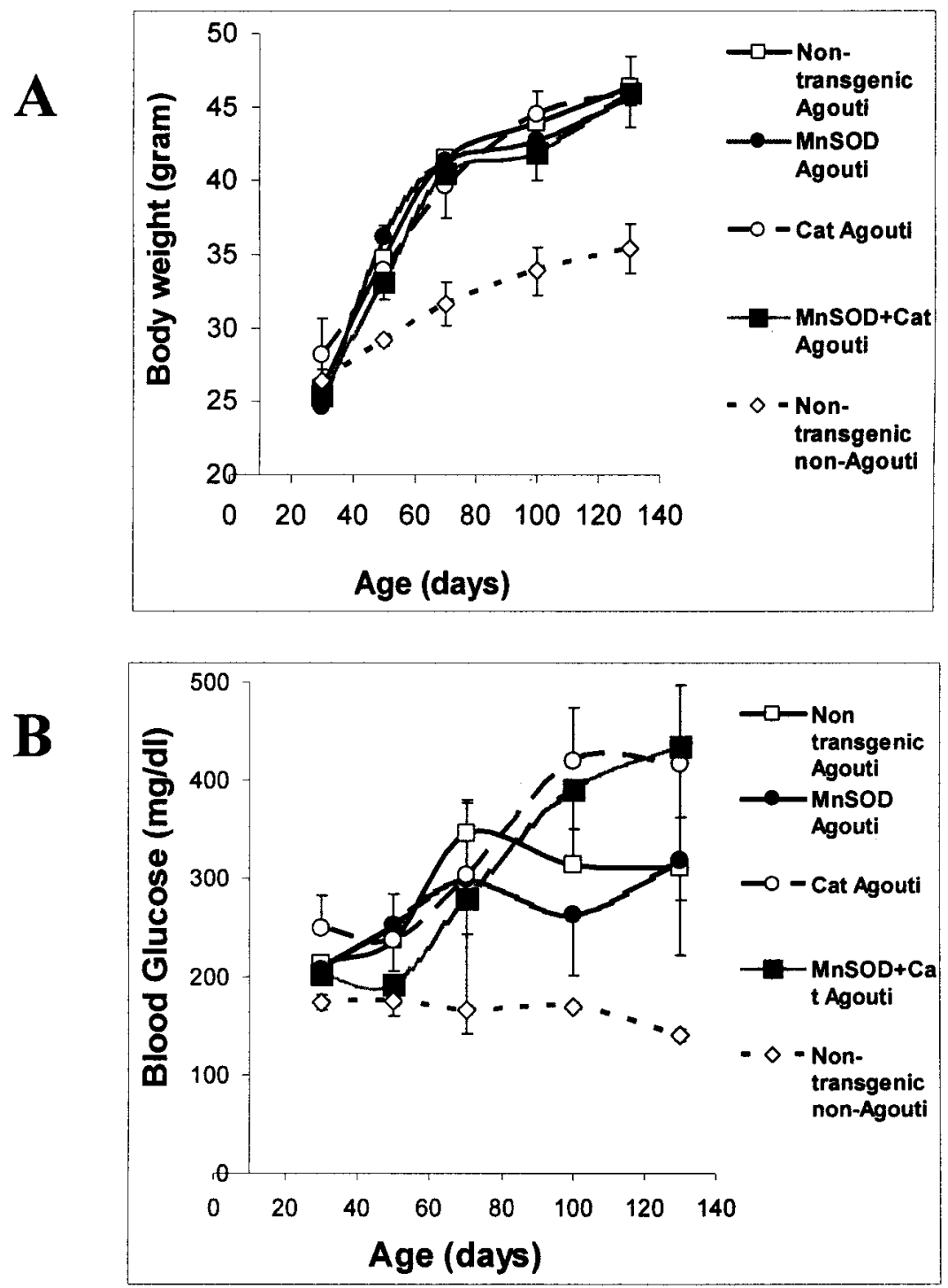

Figure 6-1. Body weight (A) and blood glucose level (B) in male F1 control and Agouti mice. Mice were produced by breeding between MnSOD+Catalase transgenic FVB mice and Agouti/KK mice. Body weight and blood glucose were measured as described in Materials and Methods. The data at each time point were mean values from at least three animals. The vertical bars indicate the standard error. Transgenic and nontransgenic Agouti mice developed similar degree of obesity. None of the antioxidant transgene prevented Agouti Type 2 diabetes ( $p>0.05$ transgenic vs. nontransgenic agouti mice by one-way ANOVA). 


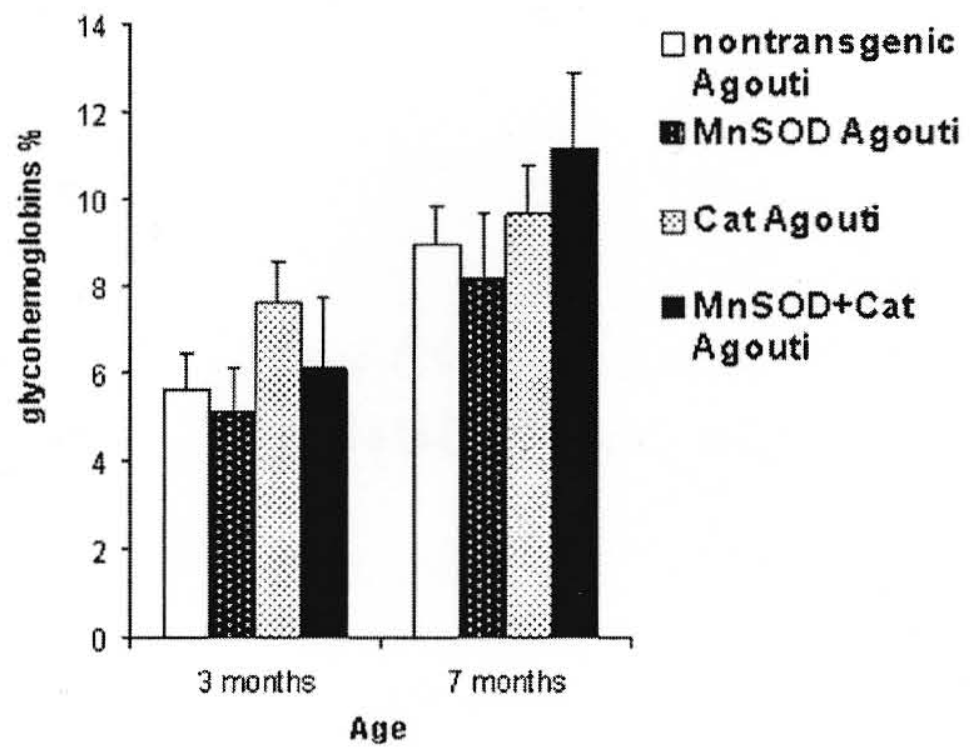

Figure 6-2. Blood glycohemoglobin content in transgenic and nontransgenic F1 Agouti mice. Each value was calculated from 2 or 3 mice per group at the age of 3 months and from 3 or 4 mice per group at the age of 7 months. Vertical bars indicate standard error of the mean. Antioxidant transgenes, either alone (MnSOD or Catalase) or in combination did not inhibit the increase of blood glycohemoglobin content ( $\mathrm{P}>0.05$ by one-way ANOVA vs. nontransgenic Agouti F1 mice). 


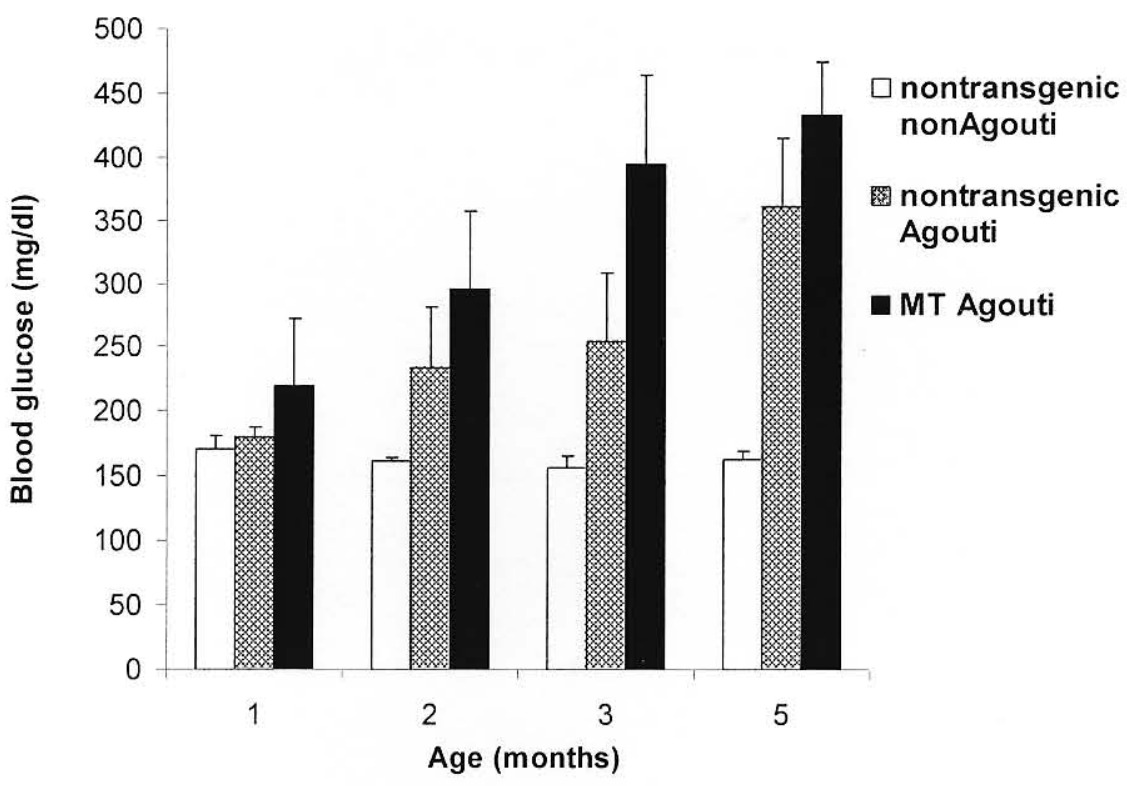

Figure 6-3. Blood glucose level in male F1 control and Agouti mice containing or not containing the MT transgene. Mice were produced by breeding HMT-1 transgenic FVB mice and Agouti/KK mice. Nonfasting blood glucose was measured as described in Materials and Methods. The data at each time point are mean values obtained from 4 to 8 animals. The vertical bars indicate the standard error. The MT transgene did not prevent Type 2 diabetes in Agouti mice ( $p>0.05$ vs. nontransgenic agouti mice by one-way ANOVA). 
Table 6-1. Frequency of diabetic mice in $\mathrm{C57} \mathrm{BL} / \mathrm{KsJ}$ mice at each generation due to the beta cell MT transgene.

\begin{tabular}{|c|c|c|c|c|c|}
\hline \multicolumn{2}{|c|}{ Times of backcross } & \multicolumn{2}{|c|}{ Transgenic } & \multicolumn{2}{|c|}{ Control } \\
\hline & & 0 & 9 & 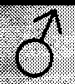 & 9 \\
\hline $\operatorname{lm} 1 \mathrm{C} 1$ & 1 & $2 / 6$ & $0 / 3$ & $0 / 2$ & $0 / 4$ \\
\hline $\operatorname{lm} 1 \mathrm{C} 4$ & 4 & $3 / 3$ & $0 / 2$ & $0 / 2$ & $0 / 2$ \\
\hline $\operatorname{Im} 1 \mathrm{C}_{5}$ & 5 & $6 / 6$ & $1 / 3$ & $0 / 2$ & $0 / 3$ \\
\hline $\operatorname{lm} 1 \mathrm{C}_{6}$ & 6 & $6 / 6$ & $3 / 6$ & $0 / 4$ & $0 / 2$ \\
\hline
\end{tabular}

Data are expressed as the number of mice that became diabetic over the total number of total mice observed in that group. $\operatorname{Im} 1 \mathrm{C} 1, \operatorname{Im} 1 \mathrm{C} 4, \operatorname{Im} 1 \mathrm{C} 5, \operatorname{Im} 1 \mathrm{C} 6$ refer to $1^{\text {st }}, 4^{\text {th }}, 5^{\text {th }}, 6^{\text {th }}$ generation, respectively of backcrossing to C $57 \mathrm{BL} / \mathrm{KsJ}$ mice. 


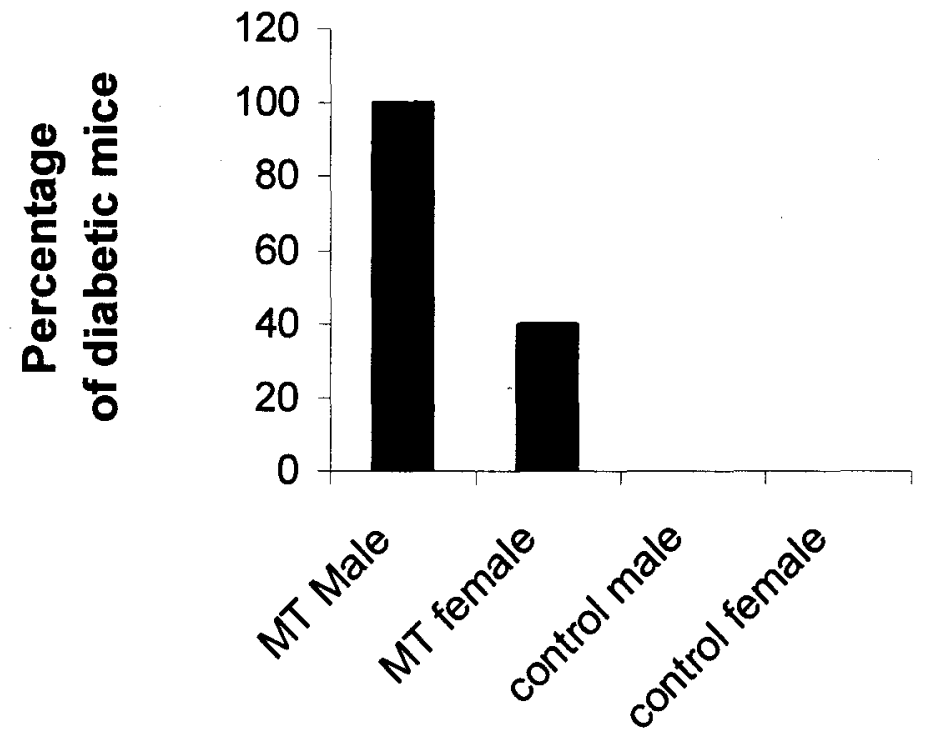

Figure 6-4. Frequency of diabetes in MT transgenic and nontransgenic C57BL/KsJ mice. At the sixth generation backcross of HMT-1 positive mice to C57BL/KsJ mice, all males and $40 \%$ of female MT transgenic mice developed severe diabetes, whereas no littermates without the MT transgene became diabetic. 


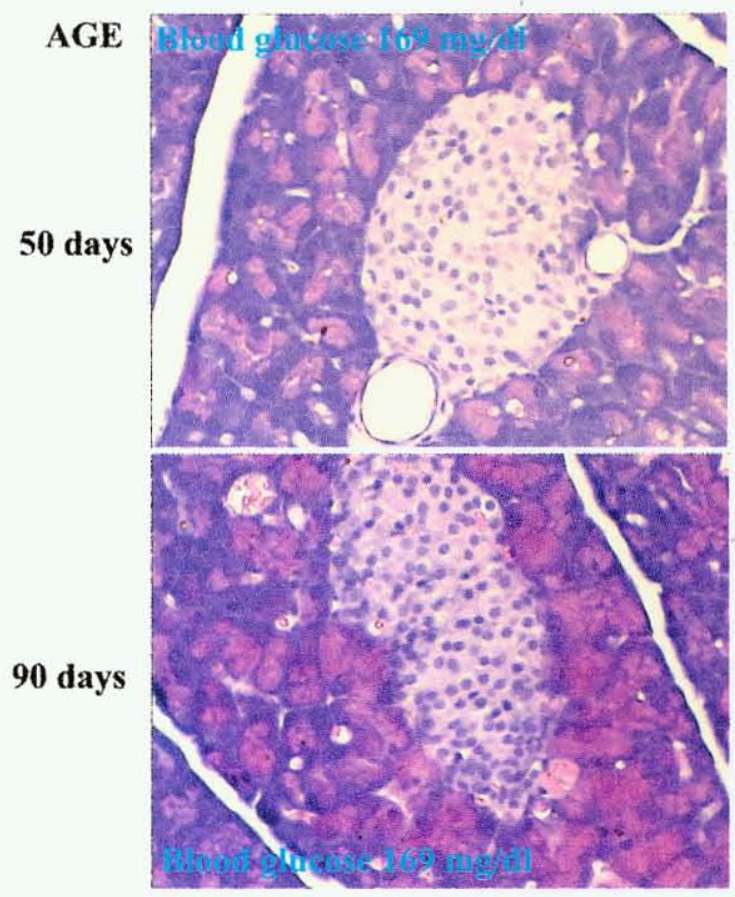

C57BLKs/J
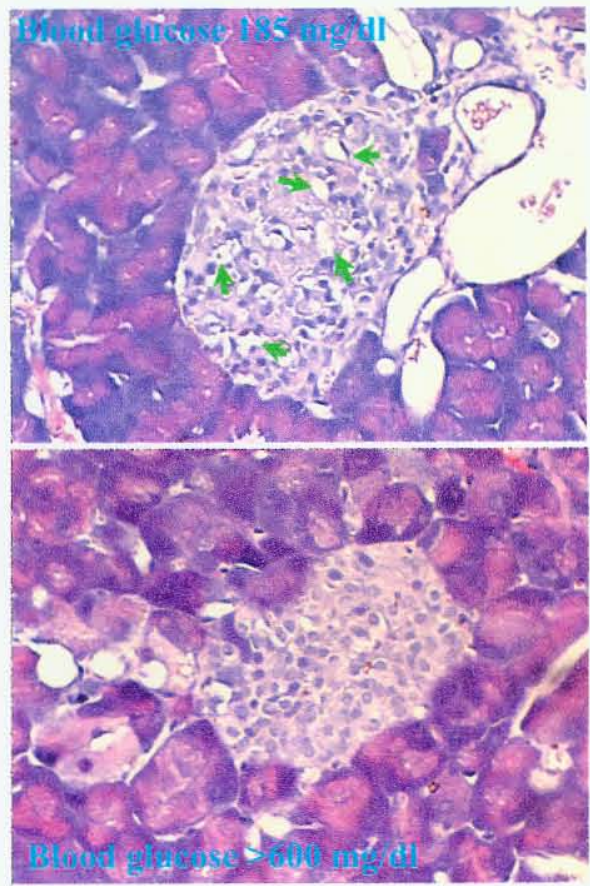

MTC57BLKs/J

Figure 6-5. Morphology of pancreatic islets in MT transgenic (MTC57BLKs/J) and nontransgenic C57BLKs/J mice. The pancreatas from different aged (50 and 90 days) animals were sectioned and stained with H\&E. MT transgenic islets showed significant islet atrophy and islet cell degeneration. Green arrows point to distinct vacuole structures commonly present in MT transgenic islets, probably left-over when beta cells undergo apoptosis. Magification $\times 200$. 


\section{SUMMARY AND DIRECTION FOR FUTURE STUDIES}

\section{Summary}

One of the main goals for diabetes treatment is to prevent pancreatic beta cell death. A large body of evidence has demonstrated that excessive ROS are formed in both Type 1 and Type 2 diabetes. Pancreatic beta cell death is mediated by an autoimmune reaction in Type 1 diabetes (Horio et al. 1994; Nomikos et al. 1989). In Type 2 diabetes beta cell damage is thought to be a consequence of long term exposure to high level of glucose and/or free fatty acids (Tanaka et al. 2002; Ihara et al. 1999). In vitro studies using defined chemical or enzymatic ROS sources have revealed that islet cells are extremely susceptible to ROS induced cell destruction (Malaisse et al. 1982). This susceptibility may be due to the fact that pancreatic beta cells contain particularly low level of expression and activity of ROS detoxifying systems compared to other cell types (Grankvist et al. 1981b; Lenzen et al. 1996). A number of studies, which were carried out mostly in vitro using insulin secreting tumor cell lines or isolated islets, have reported that elevated antioxidant protein expression in beta cells, obtained by gene transfer techniques or application of ROS scavenger compounds, protects from ROS destruction (Lortz et al. 2000; Tiedge et al. 1998; Tiedge et al. 1999). Hence the antioxidant is expected to produce a protective effect in vivo. However, the translation of this notion to in vivo studies from the in vitro results is very unclear. Attempts to use antioxidant therapy to treat animal or human diabetes have shown conflicting and questionable results (Welsh et al. 1994c; Burkart \& Kolb 1993; Piercy et al. 2000; Berglund et al. 1988; Sandstrom et al. 2002). This may be because prolonged high level, in vivo treatment with antioxidants is difficult to achieve and it is not tissue

specific. As a result the studies of this project were designed to clarify this ambiguity. The overall purpose of this project was to perform an in vivo test of whether direct antioxidant protection of pancreatic beta cells could prevent Type 1 or Type 2 diabetes. 
To achieve this aim, several lines of transgenic mice with beta cell specific expression of antioxidants including MnSOD, catalase, MnSOD plus catalase, and MT were created on the FVB strain of mice. These transgenes were specifically expressed in pancreatic beta cells at a high level but without detectable damage to normal beta cell function and structure. These transgenic animals provided us with an optimal model to perform the in vivo test of the potential effect of antioxidant therapy on diabetes.

In this study we have demonstrated that the transgenic beta cells or transgenic animals are highly resistant to oxidative damage. By measuring $\mathrm{CM}-\mathrm{H}_{2}$ DCFDA oxidation, the pancreatic islet cells containing antioxidant transgene (s) were shown to be highly effective in scavenging ROS. The transgenic islets generally survived and functioned better than control islets when exposed to various ROS insults, including $\mathrm{H}_{2} \mathrm{O}_{2}$, NO and STZ. More importantly, in vivo all of these antioxidant transgenes reduced STZ induced diabetes and beta cell morphological disruption. This protective effect was due to protection from ROS since STZ induced pancreatic beta cell oxidative damage marker nitrotyrosine was remarkably reduced in transgenic islets. Moreover, the combination of MnSOD and catalase made the beta cells more effective in scavenging some types of ROS and more resistant to STZ induced beta cell destruction and diabetes than either transgene alone. These results are consistent with published in vitro data (Lortz et al. 2000; Tiedge et al. 1998). Those in vitro studies aimed to develop a robust beta cell line with enhanced resistance to oxidative stress. Now such a goal has been achieved in a whole animal in this project. We have developed transgenic FVB mice with beta cell specific antioxidant protection that produces great resistance to ROS damage and STZ induced diabetes.

We anticipated that these antioxidant protected beta cells should also be very resistant to cytokine toxicity, and that these antioxidant transgenic mice should be protected from Type 1 and Type 2 diabetes. However, the results from the rest of the project were disappointing but interesting and probably very valuable. Unlike insulin secreting cell lines exposed to cytokines, our primary mouse beta cells with antioxidant overexpression lost cellular function and died exactly the same as control cells as indicated by insulin secretory function and islet cell metabolic activity. Cytokine induced beta cell iNOS expression and 
NO production were not inhibited by antioxidant transgene expression. These results suggest a negligible impact of ROS and antioxidants in mediation of cytokine toxicity to primary mouse beta cells.

The lack of benefit of antioxidant proteins was also true in Type 2 diabetes models. In these studies new lines of obese and diabetic mice heterozygous for the Agouti gene were produced with beta cell antioxidant transgenes. No antioxidant transgene was shown protective against Type 2 diabetes onset and development in these mice. This is the first in vivo study using transgenic animal to test whether antioxidant proteins are beneficial for prevention of Type 2 diabetes. Obviously the answer is no, at least in the Agouti obese model.

A most interesting and most unexpected finding in this study was obtained with Type 1 diabetic NOD mice and largely normal C57BL/KsJ mice. Two new lines of NOD mice congenic for beta cell MT and catalase were independently established. Very surprisingly both MT and catalase transgenes accelerated NOD diabetes onset after injection of CYP. Several potential artifacts have been ruled out that could explain this result: In vitro studies showed that metallothionein did not directly sensitize islets to CYP, in fact it provided significant protection. Prior to CYP injection pancreatic insulin, islet morphology and beta cell apoptosis were the same in transgenic and nontransgenic NOD mice. In C57BL/KsJ mice that were originally normal and euglycemic, introduction of the beta cell MT transgene impaired beta cells and caused severe diabetes as early as 40 to 50 days of age. These data demonstrate that the antioxidants themselves impair beta cell survival in CYP injected NOD mice and C57BL/KsJ mice. This implies that ROS may not only be harmful but they may also be necessary for beta cell survival under some kinds of diabetic stress, for example immune stress in NOD mice.

This new hypothesis is a challenge to the current notion that ROS act only to destroy pancreatic beta cells, and antioxidant treatment must be beneficial in treating diabetes. However our hypothesis probably is supported by emerging evidence obtained in many cell types indicating that ROS participate in cellular signal transduction, probably even in the beta cell. 
However, it needs to be pointed out that all the colonies maintained in the animal facilities of the University of Louisville by our laboratory currently are infected with mouse Parvovirus (MPV). MPV is one of the most prevalent infectious pathogens in research institutions (Besselsen et al. 2000). MPV often replicates in mitotically active tissues, such as gastrointestinal tract, lymphocytes, and tumors. MPV has high resistance to temperature and harsh environmental conditions. In vivo, MPV can remain infectious for a very long time after initial exposure, even in adult mice (Jacoby et al. 1996). In general, MPV infection causes no clinical symptoms or diseases in infected infant or adult mice, whether they are immunocompetent or severely immunocomprised (SCID mice) (Jacoby et al. 1995). But MPV infections can alter immune function. $\mathrm{CD}^{+}$and $\mathrm{CD}^{+}$cell function may be inhibited (McKisic et al. 1996; BallGoodrich et al. 1998). Lymphocytes from the spleen and popliteal lymph nodes showed inhibited function in one study (Nicklas 1999). Therefore in NOD mice MPV contamination theoretically could delay diabetes onset instead of accelerating it, as revealed in our project. However, we cannot be certain what impact MPV infection has on NOD mice and C57BLKs/J mice in our studies. Our results need to be replicated in mice free of MPV infection that are housed in pathogen-free facilities.

\section{Direction for Future Studies}

It has been recognized for long time that ROS are produced in aerobic cells as by-products during mitochondrial electron transport, by several oxidoreductases and by metal-catalyzed oxidation of metabolites (Baynes 1991; Forman \& Torres 2001; Nakamura et al. 1997). But classically they are viewed solely as a toxic evil to the aerobic cells. Recently it has been appreciated that ROS also participate in cell signal transduction as signal molecules, especially in proinflammatory cytokine and growth factor mediated cell signaling.

ROS in cytokine and growth factor mediated cell signaling. Analysis of cells in culture has demonstrated that a variety of ligands including platelet-derived growth factor (PDGF), epidermal growth 
factor (EGF), angiotensin II, as well as a host of cytokines, all trigger the rapid production of intracellular ROS. These endogenously produced ROS act as second messengers to stimulate protein kinase cascades coupled to gene expression, or control of cell cycle (Kamata \& Hirata 1999).

$\mathrm{H}_{2} \mathrm{O}_{2}$ is the first identified endogenous ROS involved in cellular signaling. In vascular smooth muscle cells, activation of PDGF caused peroxide formation which could be inhibited by intracellular expression of catalase. Antioxidant N-acetyl-cysteine inhibited the activation of PDGF receptor-coupled protein kinase cascades (Sundaresan et al. 1995). Similarly EGF signaling also involves $\mathrm{H}_{2} \mathrm{O}_{2}$ formation. In human epidermoid carcinoma cells EGF resulted in a transient increase in the intracellular concentration of ROS, measured with the oxidation-sensitive fluorescent probe $2^{\prime}, 7^{\prime}$-dichlorofluorescin diacetate and laserscanning confocal microscopy. Application of catalase by electroporation abolished DCF fluorescence and inhibited EGF-induced tyrosine phosphorylation (Bae et al. 1997). The involvement of ROS in insulin signaling has also been reported. $\mathrm{H}_{2} \mathrm{O}_{2}$ was found to have insulin-like stimulating effect on various cell types, including adipocytes, leukocytes and hepatoma cells. There are reports showing $\mathrm{H}_{2} \mathrm{O}_{2}$ increased insulin receptor kinase activity by increasing phosphorylation of the insulin receptor. A direct measurement by DCF fluorescent probes revealed that $\mathrm{H}_{2} \mathrm{O}_{2}$ was formed in human polymorphonuclear leukocytes (PMNLs) treated with insulin. This increase of $\mathrm{H}_{2} \mathrm{O}_{2}$ was enhanced by a PKC stimulator but partially inhibited by a PKC inhibitor (Spagnoli et al. 1995). Several studies have demonstrated that peroxide can mediate cytokine signaling. In primary rat astrocytes, both interleukin-1 $\beta$ and $\mathrm{H}_{2} \mathrm{O}_{2}$ promoted phosphoactivation of the $\mathrm{P}_{38}$-mitogen activated protein kinase. A direct measurement showed that $\mathrm{H}_{2} \mathrm{O}_{2}$ formation was increased in IL-1 $\beta$ treated astrocytes, which could be suppressed by the antioxidants $\mathrm{N}$-acetyl-cysteine and the nitrone-base antioxidant phenyl-N-tert-butylnitrone (PBN). Although there are no reports showing ROS involvement in beta cell signaling after cytokine or growth factor treatment, it is reasonable to expect that such kinds of signaling exist in beta cells. Both cytokine and insulin receptors are highly expressed on pancreatic beta cell membranes. Cytokines have important actions in beta cells (Maedler et al. 2002). More and more studies have shown that insulin signaling is important for beta cell function and survival. 
Protein Tyrosine Phosphatases - important targets regulated by ROS. Although many studies have indicated the presence of endogenous ROS-sensitive signal transduction pathways, the exact targets regulated by ROS are just starting to be identified, such as NF-KB, AP-1 and JNK. Among of those, protein tyrosine phosphatase (PTP) is emerging as one of the most important ROS regulated targets related to diabetes pathology. PTPs, coordinating with protein tyrosine kinases, play an important role in maintaining a normal tyrosine phosphorylation status in vivo. Aberrant tyrosine phosphorylation due to disturbance of the balance between protein tyrosine kinase and PTPs function has been implicated in the etiology of several human diseases, including cancer, diabetes and inflammation (Tonks \& Neel 2001). Many studies have shown PTPs are major targets for $\mathrm{H}_{2} \mathrm{O}_{2}$. PTPs contain an essential catalytic cysteine residue in their active sites, which is extremely reactive, with a lower pKa (about 5.5) than the pKa (about 8) of cysteine residues in most other proteins (Peters et al. 1998). The low pKa makes the thiolate anion especially susceptible to the inhibitory action of ROS by forming a sulfenic acid (Cys-sOH) intermediate. This transient intermediate could be reversed by a thiol-reducing agent such as glutathione. It has been reported $\mathrm{H}_{2} \mathrm{O}_{2}$ treatment could inhibit PTPs in vitro (Denu \& Tanner 1998) as well as in several cell lines such as HER14 cells (Sullivan et al. 1994), Jurkat T lymphocytes (Secrist et al. 1993) and HELA cells (Cunnick et al. 1998). Antioxidants such as $\mathrm{N}$-acetylcysteine (NAC) inhibits $\mathrm{H}_{2} \mathrm{O}_{2}$-induced phosphatase inactivation in glia cell lysate (Robinson et al. 1999). More importantly, there are reports showing that endogenous $\mathrm{H}_{2} \mathrm{O}_{2}$ production after insulin stimulation reversibly inactivated PTP-1B and enhanced the early insulin signal cascade (Tao et al. 2001). This reversible inhibition of PTPs could be abolished by catalase. Therefore the inhibition of PTPs by ROS has been thought to be a physiologically important event during cell signaling by insulin.

A growing body of evidence has emerged to support the concept that PTPs play an important role in obesity and diabetes. Several PTPs have been identified in major insulin-sensitive tissues, such as skeletal muscle, liver, and adipose tissue. PTP-1B-deficient mice show increased insulin sensitivity in muscle and liver, and resistance to obesity (Elchebly et al. 1999). When these knock-out mice are crossed onto ob/ob mice, improvement in blood glucose and obesity is seen. When PTP-1B antisense oligonucleotides are administered in vivo to $a b / o b$ mice, normalized blood glucose levels are obtained. However the 
overexpression of PTP-1B inhibits proximal and distal insulin signaling events. Therefore, PTP-1B is considered to be involved in the etiology of diabetes mellitus.

Insulin signaling and beta cell fate. The insulin signaling pathway is initiated by tyrosine autophosphorylation of the insulin receptor followed by several different cellular scaffold proteins binding to the autophosphorylation sties. Then the multiple tyrosine residues of a series of key post-receptor substrate proteins are phosphorylated. Most of these cellular scaffold proteins are tyrosine-phosphorylated proteins. Therefore the insulin signaling is balanced by the action of specific protein-tyrosine phosphatases (PTPs). PTPase activity, in turn, is highly regulated in vivo by oxidation/reduction reactions involving the cysteine thiol moiety required for catalysis.

Although the roles of those various insulin receptor scaffold proteins merit attention, recent work with transgenic mice suggests that many insulin responses, especially those that are associated with somatic growth and carbohydrate metabolism, are largely mediated through two insulin receptor substrate (IRS) proteins, called IRS-1 and IRS-2. In pancreatic islets, recent data has reported that IRS-2 branch of insulin/IGF signaling play a key role in beta cell survival and function (Withers et al. 1999; White 2002). In mice, both IRS-1 knock out and IRS-2 knock out mice have remarkable induced insulin resistance; however, only IRS-2 knock out mice fail to sustain compensatory insulin secretion due to decreased beta cell mass compared with wild-type and IRS-1 knock out mice. Those IRS-2 knock out mice eventually developed diabetes. These data suggest that the convergence of peripheral and islet defects around the IRS2 branch of the insulin/IGF signaling pathway may reveal the common pathway to diabetes. These data demonstrate the importance of insulin signaling for beta cell survival. We also know that insulin signaling is positively effected by ROS through inhibition of PTPs.

In type I diabetic patients and in transgenic NOD mice, we know that during immune attack the beta cells are exposed to many stressors including lymphocytes and cytokines. In response to these stimuli we propose that the pancreatic beta cell will first trigger their defense system by activating IRS-2 related cell signaling pathways. Since insulin, cytokine-IRS signaling is highly regulated by oxidant state sensitive PTPs, we suspect that both our antioxidant transgenes MT and catalase may inhibit this insulin, cytokine- 
IRS signaling pathway by scavenging cytokine induced ROS in the beta cells, and thereby suppress normal beta cell survival responses. This new hypothesis emphasizes the fact that ROS have survival stimulating roles in addition to toxic actions in the beta cell. Confirming this hypothesis may reveal new approaches to protect the beta cell and thus reduce diabetes. 


\section{REFERENCES}

Adorini L, Gregori S \& Harrison LC 2002 Understanding autoimmune diabetes: insights from mouse models. Trends Mol.Med. 8 31-38.

Andersen NA, Larsen CM \& Mandrup-Poulsen T 2000 TNFalpha and IFNgamma potentiate IL-Ibeta induced mitogen activated protein kinase activity in rat pancreatic islets of Langerhans. Diabetologia 43 1389-1396.

Andrade J, Conde M, Ramirez R, Monteseirin J, Conde J, Sobrino F \& Bedoya FJ 1996 Protection from nicotinamide inhibition of interleukin-1 beta-induced RIN cell nitric oxide formation is associated with induction of MnSOD enzyme activity. Endocrinology 137 4806-4810.

Apostolova MD, Choo KH, Michalska AE \& Tohyama C 1997 Analysis of the possible protective role of metallothionein in streptozotocin-induced diabetes using metallothionein-null mice. J.Trace Elem.Med.Biol. 11 1-7.

Appels B, Burkart V, Kantwerk-Funke G, Funda J, Kolb-Bachofen V \& Kolb H 1989 Spontaneous cytotoxicity of macrophages against pancreatic islet cells. J.Immunol. 142 3803-3808.

Auerbach AB, Norinsky R, Ho W, Losos K, Guo Q, Chatterjee S \& Joyner AL 2003 Strain-dependent differences in the efficiency of transgenic mouse production. Transgenic Res. 12 59-69.

Augstein P, Elefanty AG, Allison J \& Harrison LC 1998 Apoptosis and beta-cell destruction in pancreatic islets of NOD mice with spontaneous and cyclophosphamide-accelerated diabetes. Diabetologia 41 13811388 .

Aust SD, Chignell CF, Bray TM, Kalyanaraman B \& Mason RP 1993 Free radicals in toxicology. Toxicol.Appl.Pharmacol. 120 168-178.

Aust SD, Roerig DL \& Pederson TC 1972 Evidence for superoxide generation by NADPH-cytochrome c reductase of rat liver microsomes. Biochem.Biophys. Res.Commun. 47 1133-1137.

Azevedo-Martins AK, Lortz S, Lenzen S, Curi R, Eizirik DL \& Tiedge M 2003 Improvement of the mitochondrial antioxidant defense status prevents cytokine-induced nuclear factor-kappaB activation in insulin-producing cells. Diabetes 52 93-101.

Bach JF 1994 Insulin-dependent diabetes mellitus as an autoimmune disease. Endocr.Rev. 15 516-542.

Bae YS, Kang SW, Seo MS, Baines IC, Tekle E, Chock PB \& Rhee SG 1997 Epidermal growth factor (EGF)-induced generation of hydrogen peroxide. Role in EGF receptor-mediated tyrosine phosphorylation. J.Biol.Chem. 272 217-221.

Ball-Goodrich LJ, Leland SE, Johnson EA, Paturzo FX \& Jacoby RO 1998 Rat parvovirus type 1: the prototype for a new rodent parvovirus serogroup. J.Virol. 72 3289-3299. 
Banting FG \& Best CH 1990 Pancreatic extracts. 1922. J.Lab Clin.Med. 115 254-272.

Bartosz G 1996 Peroxynitrite: mediator of the toxic action of nitric oxide. Acta Biochim.Pol. 43 645-659.

Bartsch R, Klein D \& Summer KH 1990 The Cd-Chelex assay: a new sensitive method to determine metallothionein containing zinc and cadmium. Arch.Toxicol. 64 177-180.

Baynes JW 1991 Role of oxidative stress in development of complications in diabetes. Diabetes $40405-$ 412.

Behrens WA, Scott FW, Madere R, Trick K \& Hanna K 1986 Effect of dietary vitamin E on the vitamin E status in the BB rat during development and after the onset of diabetes. Ann.Nutr.Metab. 30 157-165.

Benderitter M, Vincent-Genod L, Pouget JP \& Voisin P 2003 The cell membrane as a biosensor of oxidative stress induced by radiation exposure: a multiparameter investigation. Radiat.Res. 159 471-483.

Benhamou PY, Moriscot C, Richard MJ, Beatrix O, Badet L, Pattou F, Kerr-Conte J, Chroboczek J, Lemarchand P \& Halimi S 1998 Adenovirus-mediated catalase gene transfer reduces oxidant stress in human, porcine and rat pancreatic islets [In Process Citation]. Diabetologia 41 1093-1100.

Berglund O, Frankel BJ \& Hellman B 1978 Development of the insulin secretory defect in genetically diabetic (db/db) mouse. Acta Endocrinol. (Copenh) 87 543-551.

Berglund O, Grankvist K, Albiin C \& Marklund SL 1988 No effect of superoxide dismutase on spontaneous development of diabetes in db/db mice. Acta Endocrinol. (Copenh) 117 99-102.

Bertera S, Crawford ML, Alexander AM, Papworth GD, Watkins SC, Robbins PD \& Trucco M 2003 Gene transfer of manganese superoxide dismutase extends islet graft function in a mouse model of autoimmune diabetes. Diabetes 52 387-393.

Besselsen DG, Wagner AM \& Loganbill JK 2000 Effect of mouse strain and age on detection of mouse parvovirus 1 by use of serologic testing and polymerase chain reaction analysis. Comp Med. 50 498-502.

Black RG, Jr., Guo Y, Ge ZD, Murphree SS, Prabhu SD, Jones WK, Bolli R \& Auchampach JA 2002 Gene dosage-dependent effects of cardiac-specific overexpression of the A3 adenosine receptor. Circ.Res. 91 165-172.

Blokh KO, Poltorak VV, Brindak OI \& Tur MI 1988 [Importance of db gene for the development of low dose streptozotocin diabetes in C57BL/KsJ mice]. Probl.Endokrinol. (Mosk) 34 73-75.

Bollheimer LC, Skelly RH, Chester MW, McGarry JD \& Rhodes CJ 1998 Chronic exposure to free fatty acid reduces pancreatic beta cell insulin content by increasing basal insulin secretion that is not compensated for by a corresponding increase in proinsulin biosynthesis translation. J.Clin.Invest 101 10941101.

Borg LA, Cagliero E, Sandler S, Welsh N \& Eizirik DL 1992 Interleukin-1 beta increases the activity of superoxide dismutase in rat pancreatic islets. Endocrinology 130 2851-2857.

Bottino R, Balamurugan AN, Bertera S, Pietropaolo M, Trucco M \& Piganelli JD 2002 Preservation of human islet cell functional mass by anti-oxidative action of a novel SOD mimic compound. Diabetes $\mathbf{5 1}$ 2561-2567.

Boyd AL \& Samid D 1993 Review: molecular biology of transgenic animals. J.Anim Sci. 71 Suppl 3 1-9. 
Brandhorst D, Brandhorst H, Zwolinski A, Nahidi F \& Bretzel RG 2001 Prevention of intraportal islet failure by a highly selective iNOS inhibitor in the pig-to-nude rat model. Transplant.Proc. 33523.

Bray TM \& Bettger WJ 1990 The physiological role of zinc as an antioxidant. Free Radic.Biol.Med. 8 281291.

Brenner HH, Burkart V, Rothe H \& Kolb H 1993 Oxygen radical production is increased in macrophages from diabetes prone BB rats. Autoimmunity. 15 93-98.

Brigelius-Flohe R 1999 Tissue-specific functions of individual glutathione peroxidases. Free Radic.Biol.Med. 27 951-965.

Brooks PJ 1997 DNA damage, DNA repair, and alcohol toxicity--a review [In Process Citation]. Alcohol Clin.Exp.Res. 21 1073-1082.

Bultman SJ, Michaud EJ \& Woychik RP 1992 Molecular characterization of the mouse agouti locus. Cell 71 1195-1204.

Burkart V, Koike T, Brenner HH \& Kolb H 1992 Oxygen radicals generated by the enzyme xanthine oxidase lyse rat pancreatic islet cells in vitro. Diabetologia 35 1028-1034.

Burkart V \& Kolb H 1993 Protection of islet cells from inflammatory cell death in vitro.

Clin.Exp.Immunol. 93 273-278.

Buse, J. B., Polonsky, K. S., and Burant Charles F. Williams textbook of endocrinology: (section 29) Type 2 diabetes mellitus. Williams textbook of endocrinology , 1427-1483. 2003.

Ref Type: Generic

Buttke TM \& Sandstrom PA 1994 Oxidative stress as a mediator of apoptosis. Immunol.Today 15 7-10.

Byth HA, Mchunu BI, Dubery IA \& Bornman L 2001 Assessment of a simple, non-toxic Alamar blue cell survival assay to monitor tomato cell viability. Phytochem.Anal. 12 340-346.

Cai L, Klein JB \& Kang YJ 2000 Metallothionein inhibits peroxynitrite-induced DNA and lipoprotein damage. J.Biol.Chem. 275 38957-38960.

Cardozo AK, Heimberg H, Heremans Y, Leeman R, Kutlu B, Kruhoffer M, Orntoft T \& Eizirik DL 2001 A comprehensive analysis of cytokine-induced and nuclear factor-kappa B- dependent genes in primary rat pancreatic beta-cells. J.Biol.Chem. 276 48879-48886.

Cardozo AK, Proost P, Gysemans C, Chen MC, Mathieu C \& Eizirik DL 2003 IL-1beta and IFN-gamma induce the expression of diverse chemokines and IL-15 in human and rat pancreatic islet cells, and in islets from pre-diabetic NOD mice. Diabetologia 46 255-266.

Carlson EC, Audette JL, Klevay LM, Nguyen H \& Epstein PN 1997 Ultrastructural and functional analyses of nephropathy in calmodulin- induced diabetic transgenic mice. Anat.Rec. 247 9-19.

Chua S Jr, Liu SM, Li Q, Yang L, Thassanapaff VT \& Fisher P 2002 Differential beta cell responses to hyperglycaemia and insulin resistance in two novel congenic strains of diabetes (FVB- Lepr (db)) and obese (DBA- Lep (ob)) mice. Diabetologia 45 976-990.

Chubatsu LS \& Meneghini R 1993 Metallothionein protects DNA from oxidative damage. Biochem.J. 291 (Pt 1) 193-198. 
Church SL, Grant JW, Ridnour LA, Oberley LW, Swanson PE, Meltzer PS \& Trent JM 1993 Increased manganese superoxide dismutase expression suppresses the malignant phenotype of human melanoma cells. Proc.Natl.Acad.Sci.U.S.A 90 3113-3117.

Cnop M, Hannaert JC, Hoorens A, Eizirik DL \& Pipeleers DG 2001 Inverse relationship between cytotoxicity of free fatty acids in pancreatic islet cells and cellular triglyceride accumulation. Diabetes $\mathbf{5 0}$ 1771-1777.

Contrera JF \& DeGeorge JJ 1998 In vivo transgenic bioassays and assessment of the carcinogenic potential of pharmaceuticals. Environ.Health Perspect. 106 Suppl 1 71-80.

Contreras JL, Smyth CA, Bilbao G, Eckstein C, Young CJ, Thompson JA, Curiel DT \& Eckhoff DE 2003 Coupling endoplasmic reticulum stress to cell death program in isolated human pancreatic islets: effects of gene transfer of Bcl-2. Transpl.Int.

Copeland NG, Jenkins NA \& Court DL 2001 Recombineering: a powerful new tool for mouse functional genomics. Nat.Rev.Genet. 2 769-779.

Corbett JA, Sweetland MA, Wang JL, Lancaster JRJ \& McDaniel ML 1993 Nitric oxide mediates cytokine-induced inhibition of insulin secretion by human islets of Langerhans. Proc.Natl.Acad.Sci.U.S.A. 90 1731-1735.

Courtade M, Carrera G, Paternain JL, Martel S, Carre PC, Folch J \& Pipy B 1998 Metallothionein expression in human lung and its varying levels after lung transplantation. Toulouse Lung Transplantation Group. Chest 113 371-378.

Cunnick JM, Dorsey JF, Mei L \& Wu J 1998 Reversible regulation of SHP-1 tyrosine phosphatase activity by oxidation. Biochem.Mol.Biol.Int. 45 887-894.

Dandona P, Thusu K, Cook S, Snyder B, Makowski J, Armstrong D \& Nicotera T 1996 Oxidative damage to DNA in diabetes mellitus. Lancet 347 444-445.

Dandoy-Dron F, Itier JM, Monthioux E, Bucchini D \& Jami J 1995 Tissue-specific expression of the rat insulin 1 gene in vivo requires both the enhancer and promoter regions. Differentiation 58 291-295.

Dandoy-Dron F, Monthioux E, Jami J \& Bucchini D 1991 Regulatory regions of rat insulin I gene necessary for expression in transgenic mice. Nucleic Acids Res. 19 4925-4930.

de-Mello MA, Flodstrom M \& Eizirik DL 1996 Ebselen and cytokine-induced nitric oxide synthase expression in insulin-producing cells. Biochem.Pharmacol. 52 1703-1709.

Deisseroth A \& Dounce AL 1970 Catalase: Physical and chemical properties, mechanism of catalysis, and physiological role. Physiol Rev. 50 319-375.

del Rio LA, Sandalio LM, Palma JM, Bueno P \& Corpas FJ 1992 Metabolism of oxygen radicals in peroxisomes and cellular implications. Free Radic.Biol.Med. 13 557-580.

Delaney CA, Pavlovic D, Hoorens A, Pipeleers DG \& Eizirik DL 1997 Cytokines induce deoxyribonucleic acid strand breaks and apoptosis in human pancreatic islet cells. Endocrinology 138 2610-2614.

Denu JM \& Tanner KG 1998 Specific and reversible inactivation of protein tyrosine phosphatases by hydrogen peroxide: evidence for a sulfenic acid intermediate and implications for redox regulation. Biochemistry 37 5633-5642. 
Devanesan P, Ariese F, Jankowiak R, Small GJ, Rogan EG \& Cavalieri EL 1999 A novel method for the isolation and identification of stable DNA adducts formed by Dibenzo[a,l]pyrene and Dibenzo[a,l]pyrene 11, 12-dihydrodiol 13,14-epoxides in vitro. Chem.Res. Toxicol. 12 796-801.

Dmitriev LF 2001 Activity of key enzymes in microsomal and mitochondrial membranes depends on the redox reactions involving lipid radicals. Membr. Cell Biol. 14 649-662.

Drash AL, Rudert WA, Borquaye S, Wang R \& Lieberman I 1988 Effect of probucol on development of diabetes mellitus in BB rats. Am.J.Cardiol. 62 27B-30B.

Dutta S, Gannon M, Peers B, Wright C, Bonner-Weir S \& Montminy M 2001 PDX:PBX complexes are required for normal proliferation of pancreatic cells during development. Proc.Natl.Acad.Sci.U.S.A 98 1065-1070.

Eisenbarth, G. S., Polonsky, K. S., and Buse, J. B. Williams textbook of endocrinology: (Section 30) Type 1 diabetes mellitus. Williams textbook of endocrinology, 1485-1508. 2003.

Ref Type: Generic

Eizirik DL \& Darville MI 2001 beta-cell apoptosis and defense mechanisms: lessons from type 1 diabetes. Diabetes 50 Suppl 1 S64-S69.

Eizirik DL, Flodstrom M, Karlsen AE \& Welsh N 1996 The harmony of the spheres: inducible nitric oxide synthase and related genes in pancreatic beta cells. Diabetologia 39 875-890.

Eizirik DL, Pipeleers DG, Ling Z, Welsh N, Hellerstrom C \& Andersson A 1994a Major species differences between humans and rodents in the susceptibility to pancreatic beta-cell injury.

Proc.Natl.Acad.Sci.U.S.A. 91 9253-9256.

Eizirik DL, Sandler S, Welsh N, Cetkovic-Cvrlje M, Nieman A, Geller DA, Pipeleers DG, Bendtzen K \& Hellerstrom C 1994b Cytokines suppress human islet function irrespective of their effects on nitric oxide generation. J.Clin.Invest 93 1968-1974.

Elchebly M, Payette P, Michaliszyn E, Cromlish W, Collins S, Loy AL, Normandin D, Cheng A, HimmsHagen J, Chan CC, Ramachandran C, Gresser MJ, Tremblay ML \& Kennedy BP 1999 Increased insulin sensitivity and obesity resistance in mice lacking the protein tyrosine phosphatase-1B gene. Science 283 1544-1548.

Epstein PN, Boschero AC, Atwater I, Cai X \& Overbeek PA 1992 Expression of yeast hexokinase in pancreatic beta cells of transgenic mice reduces blood glucose, enhances insulin secretion, and decreases diabetes. Proc.Natl.Acad.Sci.U.S.A. 89 12038-12042.

Epstein PN, Overbeek PA \& Means AR 1989 Calmodulin-induced early-onset diabetes in transgenic mice. Cell 58 1067-1073.

Esposti MD, Ngo A \& Myers MA 1996 Inhibition of mitochondrial complex I may account for IDDM induced by intoxication with the rodenticide Vacor. Diabetes 45 1531-1534.

Estabrook RW, Kawano S, Werringloer J, Kuthan H, Tsuji H, Graf H \& Ullrich V 1979 Oxycytochrome P450: its breakdown to superoxide for the formation of hydrogen peroxide. Acta Biol.Med.Ger 38 423-434.

Flodstrom M \& Eizirik DL 1997 Interferon-gamma-induced interferon regulatory factor-1 (IRF-1) expression in rodent and human islet cells precedes nitric oxide production. Endocrinology 138 2747-2753. 
Flodstrom M, Tyrberg B, Eizirik DL \& Sandler S 1999 Reduced sensitivity of inducible nitric oxide synthase-deficient mice to multiple low-dose streptozotocin-induced diabetes. Diabetes 48 706-713.

Forman HJ \& Torres M 2001 Redox signaling in macrophages. Mol.Aspects Med. 22 189-216.

Forman HJ, Torres M \& Fukuto J 2002 Redox signaling. Mol.Cell Biochem. 234-235 49-62.

Foulis AK 1996 The pathology of the endocrine pancreas in type 1 (insulin-dependent) diabetes mellitus. APMIS 104 161-167.

Freeman BA \& Crapo JD 1982 Biology of disease: free radicals and tissue injury. Lab Invest 47 412-426.

Fukuda M, Ikegami H, Kawaguchi Y, Sano T \& Ogihara T 1995 Antioxidant, probucol, can inhibit the generation of hydrogen peroxide in islet cells induced by macrophages and prevent islet cell destruction in NOD mice. Biochem.Biophys.Res.Commun. 209 953-958.

Gille L, Schott-Ohly P, Friesen N, Schulte im WS, Udilova N, Nowl H \& Gleichmann H 2002 Generation of hydroxyl radicals mediated by streptozotocin in pancreatic islets of mice in vitro. Pharmacol.Toxicol. 90 317-326.

Gloyn AL 2003 The search for type 2 diabetes genes. Ageing Res.Rev. 2 111-127.

Gold G, Manning M, Heldt A, Nowlain R, Pettit JR \& Grodsky GM 1981 Diabetes induced with multiple subdiabetogenic doses of streptozotocin: lack of protection by exogenous superoxide dismutase. Diabetes 30 634-638.

Gonzalez RJ \& Tarloff JB 2001 Evaluation of hepatic subcellular fractions for Alamar blue and MTT reductase activity. Toxicol.In Vitro 15 257-259.

Gopaul NK, Anggard EE, Mallet AI, Betteridge DJ, Wolff SP \& Nourooz-Zadeh J 1995 Plasma 8-epiPGF2 alpha levels are elevated in individuals with non- insulin dependent diabetes mellitus. FEBS Lett. 368 225-229.

Gordon JW, Scangos GA, Plotkin DJ, Barbosa JA \& Ruddle FH 1980 Genetic transformation of mouse embryos by microinjection of purified DNA. Proc.Natl.Acad.Sci.U.S.A 77 7380-7384.

Gordon, JW. Production of Transgenic Mice. Methods Enzymol. 225, 747. 1993.

Ref Type: Generic

Gotoh M, Maki T, Kiyoizumi T, Satomi S \& Monaco AP 1985 An improved method for isolation of mouse pancreatic islets. Transplantation 40 437-438.

Grankvist K, Marklund S \& Taljedal IB 1981a Superoxide dismutase is a prophylactic against alloxan diabetes. Nature 294 158-160.

Grankvist K, Marklund SL \& Taljedal IB 1981b CuZn-superoxide dismutase, Mn-superoxide dismutase, catalase and glutathione peroxidase in pancreatic islets and other tissues in the mouse. Biochem.J. $199393-$ 398.

Griffith, O. W. Glutathione and glutatione disulfide. Bergmeyer, H. U., Bergmeyer, J., and Grabl, M. [8], 521-529. 1986. Deerfield Beach, FLA, Verlag Chemie. Methods of enzymatic analysis.

Ref Type: Serial (Book,Monograph) 
Groves JT \& Wang CC 2000 Nitric oxide synthase: models and mechanisms. Curr.Opin.Chem.Biol. 4 687695.

Gutteridge JM \& Halliwell B 1990 The measurement and mechanism of lipid peroxidation in biological systems. Trends.Biochem.Sci. 15 129-135.

Halliwell B \& Gutteridge JM 1995 The definition and measurement of antioxidants in biological systems. Free Radic.Biol.Med. 18 125-126.

Hammer RE, Pursel VG, Rexroad CE, Jr., Wall RJ, Bolt DJ, Ebert KM, Palmiter RD \& Brinster RL 1985 Production of transgenic rabbits, sheep and pigs by microinjection. Nature 315 680-683.

Harada M \& Makino S 1984 Promotion of spontaneous diabetes in non-obese diabetes-prone mice by cyclophosphamide. Diabetologia 27 604-606.

Harding HP \& Ron D 2002 Endoplasmic reticulum stress and the development of diabetes: a review. Diabetes 51 Suppl 3 S455-S461.

Harel S \& Kanner J 1988 The generation of ferryl or hydroxyl radicals during interaction of haemproteins with hydrogen peroxide. Free Radic.Res.Commun. 5 21-33.

Hawa MI, Beyan H, Buckley LR \& Leslie RD 2002 Impact of genetic and non-genetic factors in type 1 diabetes. Am.J.Med.Genet. 115 8-17.

Hayden MR \& Tyagi SC 2002 Islet redox stress: the manifold toxicities of insulin resistance, metabolic syndrome and amylin derived islet amyloid in type 2 diabetes mellitus. JOP. 3 86-108.

Heimberg H, Heremans Y, Jobin C, Leemans R, Cardozo AK, Darville M \& Eizirik DL 2001 Inhibition of cytokine-induced NF-kappaB activation by adenovirus- mediated expression of a NF-kappaB superrepressor prevents beta-cell apoptosis. Diabetes 50 2219-2224.

Heineke EW, Johnson MB, Dillberger JE \& Robinson KM 1993 Antioxidant MDL 29,311 prevents diabetes in nonobese diabetic and multiple low-dose STZ-injected mice. Diabetes 42 1721-1730.

Hirota K, Nishiyama A \& Yodoi J 1999 [Reactive oxygen intermediates, thioredoxin, and Ref-1 as effector molecules in cellular signal transduction]. Tanpakushitsu Kakusan Koso 44 2414-2419.

Hoeflich A, Wu M, Mohan S, Foll J, Wanke R, Froehlich T, Arnold GJ, Lahm H, Kolb HJ \& Wolf E 1999 Overexpression of insulin-like growth factor-binding protein-2 in transgenic mice reduces postnatal body weight gain. Endocrinology 140 5488-5496.

Hohmeier HE, Thigpen A, Tran VV, Davis R \& Newgard CB 1998 Stable expression of manganese superoxide dismutase (MnSOD) in insulinoma cells prevents IL-1beta- induced cytotoxicity and reduces nitric oxide production. J.Clin.Invest. 101 1811-1820.

Horio F, Fukuda M, Katoh H, Petruzzelli M, Yano N, Rittershaus C, Bonner-Weir S \& Hattori M 1994 Reactive oxygen intermediates in autoimmune islet cell destruction of the NOD mouse induced by peritoneal exudate cells (rich in macrophages) but not T cells. Diabetologia 37 22-31.

Hotta M, Tashiro F, Ikegami H, Niwa H, Ogihara T, Yodoi J \& Miyazaki J 1998 Pancreatic beta cellspecific expression of thioredoxin, an antioxidative and antiapoptotic protein, prevents autoimmune and streptozotocin-induced diabetes. J.Exp.Med. \%19;188 1445-1451.

Hurst JK 2002 Whence nitrotyrosine? J.Clin.Invest 109 1287-1289. 
Igel M, Becker W, Herberg L \& Joost HG 1997 Hyperleptinemia, leptin resistance, and polymorphic leptin receptor in the New Zealand obese mouse. Endocrinology 138 4234-4239.

Ihara Y, Toyokuni S, Uchida K, Odaka H, Tanaka T, Ikeda H, Hiai H, Seino Y \& Yamada Y 1999 Hyperglycemia causes oxidative stress in pancreatic beta-cells of GK rats, a model of type 2 diabetes. Diabetes 48 927-932.

Ihara Y, Yamada Y, Toyokuni S, Miyawaki K, Ban N, Adachi T, Kuroe A, Iwakura T, Kubota A, Hiai H \& Seino Y 2000 Antioxidant alpha-tocopherol ameliorates glycemic control of GK rats, a model of type 2 diabetes. FEBS Lett. 473 24-26.

Iles KE \& Forman HJ 2002 Macrophage signaling and respiratory burst. Immunol.Res. 26 95-105.

Ishiguro H, Sawada H, Nishii K, Yamada K \& Nagatsu T 2001 [Huntington's disease model mouse and neuronal cell death]. No To Shinkei 53 829-837.

Iwahashi H, Hanafusa T, Eguchi Y, Nakajima H, Miyagawa J, Itoh N, Tomita K, Namba M, Kuwajima M, Noguchi T, Tsujimoto Y \& Matsuzawa Y 1996 Cytokine-induced apoptotic cell death in a mouse pancreatic beta-cell line: inhibition by Bcl-2. Diabetologia 39 530-536.

Jacobson MD 1996 Reactive oxygen species and programmed cell death. Trends Biochem.Sci. 21 83-86.

Jacoby RO, Ball-Goodrich LJ, Besselsen DG, McKisic MD, Riley LK \& Smith AL 1996 Rodent parvovirus infections. Lab Anim Sci. 46 370-380.

Jacoby RO, Johnson EA, Ball-Goodrich L, Smith AL \& McKisic MD 1995 Characterization of mouse parvovirus infection by in situ hybridization. J.Virol. 69 3915-3919.

Jegstrup I, Thon R, Hansen AK \& Hoitinga MR 2003 Characterization of transgenic mice--a comparison of protocols for welfare evaluation and phenotype characterization of mice with a suggestion on a future certificate of instruction. Lab Anim 37 1-9.

Ji H, Korganow AS, Mangialaio S, Hoglund P, Andre I, Luhder F, Gonzalez A, Poirot L, Benoist C \& Mathis D 1999 Different modes of pathogenesis in T-cell-dependent autoimmunity: clues from two TCR transgenic systems. Immunol.Rev. 169 139-146.

Jialal I, Devaraj S \& Venugopal SK 2002 Oxidative stress, inflammation, and diabetic vasculopathies: the role of alpha tocopherol therapy. Free Radic. Res. 36 1331-1336.

Johansson LH \& Borg LA 1988 A spectrophotometric method for determination of catalase activity in small tissue samples. Anal.Biochem. 174 331-336.

Jones BH, Kim JH, Zemel MB, Woychik RP, Michaud EJ, Wilkison WO \& Moustaid N 1996 Upregulation of adipocyte metabolism by agouti protein: possible paracrine actions in yellow mouse obesity. Am.J.Physiol 270 E192-E196.

Kadokawa H, Briegel JR, Blackberry MA, Blache D, Martin GB \& Adams NR 2003 Reproduction and plasma concentrations of leptin, insulin and insulin-like growth factor 1 in growth-hormone-transgenic female sheep before and after artificial insemination. Reprod.Fertil.Dev. 15 47-53.

Kamata H \& Hirata H 1999 Redox regulation of cellular signalling. Cell Signal. 11 1-14. 
Kaneto H, Fujii J, Myint T, Miyazawa N, Islam KN, Kawasaki Y, Suzuki K, Nakamura M, Tatsumi H, Yamasaki Y \& Taniguchi N 1996 Reducing sugars trigger oxidative modification and apoptosis in pancreatic beta-cells by provoking oxidative stress through the glycation reaction. Biochem.J. 320 855-863.

Kaneto H, Kajimoto Y, Miyagawa J, Matsuoka T, Fujitani Y, Umayahara Y, Hanafusa T, Matsuzawa Y, Yamasaki Y \& Hori M 1999 Beneficial effects of antioxidants in diabetes: possible protection of pancreatic beta-cells against glucose toxicity. Diabetes $\mathbf{4 8}$ 2398-2406.

Kang YJ, Chen Y \& Epstein PN 1996 Suppression of doxorubicin cardiotoxicity by overexpression of catalase in the heart of transgenic mice. J.Biol.Chem. 271 12610-12616.

Karnovsky MJ 1965 A formaldehyde-glutaraldehyde fixitive for high osmololity for use in electron microscopy. J.Cell Biol. 35 137A-138A.

Kennedy MC, Gan T, Antholine WE \& Petering DH 1993 Metallothionein reacts with Fe2+ and NO to form products with A $\mathrm{g}=2.039 \mathrm{ESR}$ signal. Biochem.Biophys.Res.Commun. 196 632-635.

Kessler L, Jesser C, Lombard Y, Karsten V, Belcourt A, Pinget M \& Poindron P 1996 Cytotoxicity of peritoneal murine macrophages against encapsulated pancreatic rat islets: in vivo and in vitro studies. J.Leukoc.Biol. 60 729-736.

Kim KH, Rodriguez AM, Carrico PM \& Melendez JA 2001 Potential mechanisms for the inhibition of tumor cell growth by manganese superoxide dismutase. Antioxid.Redox. Signal. 3 361-373.

Kita T, Nagano Y, Yokode M, Ishii K, Kume N, Ooshima A, Yoshida H \& Kawai C 1987 Probucol prevents the progression of atherosclerosis in Watanabe heritable hyperlipidemic rabbit, an animal model for familial hypercholesterolemia. Proc.Natl.Acad.Sci.U.S.A. 84 5928-5931.

Klebig ML, Wilkinson JE, Geisler JG \& Woychik RP 1995 Ectopic expression of the agouti gene in transgenic mice causes obesity, features of type II diabetes, and yellow fur. Proc.Natl.Acad.Sci.U.S.A 92 4728-4732.

Kleemann R, Rothe H, Kolb-Bachofen V, Xie QW, Nathan C, Martin S \& Kolb H 1993 Transcription and translation of inducible nitric oxide synthase in the pancreas of prediabetic BB rats. FEBS Lett. 328 9-12.

Koppenol WH, Moreno JJ, Pryor WA, Ischiropoulos H \& Beckman JS 1992 Peroxynitrite, a cloaked oxidant formed by nitric oxide and superoxide. Chem.Res. Toxicol. 5 834-842.

Koster JC, Marshall BA, Ensor N, Corbett JA \& Nichols CG 2000 Targeted overactivity of beta cell K (ATP) channels induces profound neonatal diabetes. Cell 100 645-654.

Kroncke KD, Fehsel K, Sommer A, Rodriguez ML \& Kolb-Bachofen V 1995 Nitric oxide generation during cellular metabolization of the diabetogenic N-methyl-N-nitroso-urea streptozotozin contributes to islet cell DNA damage. Biol.Chem.Hoppe Seyler 376 179-185.

Kubisch HM, Wang J, Bray TM \& Phillips JP 1997 Targeted overexpression of Cu/Zn superoxide dismutase protects pancreatic beta-cells against oxidative stress. Diabetes 46 1563-1566.

Kumari MV, Hiramatsu M \& Ebadi M 1998 Free radical scavenging actions of metallothionein isoforms I and II. Free Radic.Res 29 93-101.

Laybutt DR, Kaneto H, Hasenkamp W, Grey S, Jonas JC, Sgroi DC, Groff A, Ferran C, Bonner-Weir S, Sharma A \& Weir GC 2002 Increased expression of antioxidant and antiapoptotic genes in islets that may contribute to beta-cell survival during chronic hyperglycemia. Diabetes 51 413-423. 
Lazo JS, Kuo SM, Woo ES \& Pitt BR 1998 The protein thiol metallothionein as an antioxidant and protectant against antineoplastic drugs. Chem.Biol.Interact. 111-112:255-62 255-262.

LeDoux SP, Woodley SE, Patton NJ \& Wilson GL 1986 Mechanisms of nitrosourea-induced beta-cell damage. Alterations in DNA. Diabetes 35 866-872.

Lee Y, Hirose H, Zhou YT, Esser V, McGarry JD \& Unger RH 1997 Increased lipogenic capacity of the islets of obese rats: a role in the pathogenesis of NIDDM. Diabetes 46 408-413.

Leffert JD, Newgard CB, Okamoto H, Milburn JL \& Luskey KL 1989 Rat amylin: cloning and tissuespecific expression in pancreatic islets. Proc.Natl.Acad.Sci.U.S.A. 86 3127-3130.

Leiter EH, Coleman DL, Eisenstein AB \& Strack I 1981 Dietary control of pathogenesis in C57BL/KsJ $\mathrm{db} / \mathrm{db}$ diabetes mice. Metabolism 30 554-562.

Leiter EH, Coleman DL, Ingram DK \& Reynolds MA 1983 Influence of dietary carbohydrate on the induction of diabetes in C57BL/KsJ-db/db diabetes mice. J.Nutr. 113 184-195.

Leiter EH, Le PH \& Coleman DL 1987a Susceptibility to db gene and streptozotocin-induced diabetes in C57BL mice: control by gender-associated, MHC-unlinked traits. Immunogenetics 26 6-13.

Leiter EH, Prochazka M \& Coleman DL 1987b The non-obese diabetic (NOD) mouse. Am.J.Pathol. 128 380-383.

Lenaz G, Bovina C, D'Aurelio M, Fato R, Formiggini G, Genova ML, Giuliano G, Pich MM, Paolucci U, Castelli GP \& Ventura B 2002 Role of mitochondria in oxidative stress and aging. Ann.N.Y.Acad.Sci. 959 199-213.

Lenzen S, Drinkgern J \& Tiedge M 1996 Low antioxidant enzyme gene expression in pancreatic islets compared with various other mouse tissues. Free Radic.Biol.Med. 20 463-466.

Li H, Burkhardt C, Heinrich UR, Brausch I, Xia N \& Forstermann U 2003 Histamine upregulates gene expression of endothelial nitric oxide synthase in human vascular endothelial cells. Circulation $1072348-$ 2354.

Li N, Oberley TD, Oberley LW \& Zhong W 1998 Inhibition of cell growth in NIH/3T3 fibroblasts by overexpression of manganese superoxide dismutase: mechanistic studies. J.Cell Physiol 175 359-369.

Li N, Zhai Y \& Oberley TD 1999 Two distinct mechanisms for inhibition of cell growth in human prostate carcinoma cells with antioxidant enzyme imbalance. Free Radic.Biol.Med. 26 1554-1568.

Ling Z, Van de CM, Eizirik DL \& Pipeleers DG 2000 Interleukin-1 beta-induced alteration in a beta-cell phenotype can reduce cellular sensitivity to conditions that cause necrosis but not to cytokine-induced apoptosis. Diabetes 49 340-345.

Liochev SL 1996 The role of iron-sulfur clusters in in vivo hydroxyl radical production. Free Radic. Res. 25 369-384.

Listenberger LL, Ory DS \& Schaffer JE 2001 Palmitate-induced apoptosis can occur through a ceramideindependent pathway. J.Biol.Chem. 276 14890-14895.

Liu D, Pavlovic D, Chen MC, Flodstrom M, Sandler S \& Eizirik DL 2000a Cytokines induce apoptosis in beta-cells isolated from mice lacking the inducible isoform of nitric oxide synthase (iNOS-/-). Diabetes 49 1116-1122. 
Liu K, Paterson AJ, Chin E \& Kudlow JE 2000b Glucose stimulates protein modification by O-linked GlcNAc in pancreatic beta cells: linkage of O-linked GlcNAc to beta cell death. Proc.Natl.Acad.Sci.U.S.A $972820-2825$.

Liu Y, Rabinovitch A, Suarez-Pinzon W, Muhkerjee B, Brownlee M, Edelstein D \& Federoff HJ 1996 Expression of the bcl-2 gene from a defective HSV-1 amplicon vector protects pancreatic beta-cells from apoptosis. Hum.Gene Ther. 7 1719-1726.

Lo D, Burkly LC, Widera G, Cowing C, Flavell RA, Palmiter RD \& Brinster RL 1988 Diabetes and tolerance in transgenic mice expressing class II MHC molecules in pancreatic beta cells. Cell 53 159-168.

Lorenzo A, Razzaboni B, Weir GC \& Yankner BA 1994 Pancreatic islet cell toxicity of amylin associated with type-2 diabetes mellitus. Nature 368 756-760.

Lortz S \& Tiedge M 2003 Sequential inactivation of reactive oxygen species by combined overexpression of SOD isoforms and catalase in insulin-producing cells. Free Radic.Biol.Med. 34 683-688.

Lortz S, Tiedge M, Nachtwey T, Karlsen AE, Nerup J \& Lenzen S 2000 Protection of insulin-producing RINm5F cells against cytokine-mediated toxicity through overexpression of antioxidant enzymes. Diabetes $491123-1130$.

Maclaren NK, Elder ME, Robbins VW \& Riley WJ 1983 Autoimmune diatheses and T lymphocyte immunoincompetences in BB rats. Metabolism 32 92-96.

Maedler K, Fontana A, Ris F, Sergeev P, Toso C, Oberholzer J, Lehmann R, Bachmann F, Tasinato A, Spinas GA, Halban PA \& Donath MY 2002 FLIP switches Fas-mediated glucose signaling in human pancreatic beta cells from apoptosis to cell replication. Proc.Natl.Acad.Sci.U.S.A 99 8236-8241.

Makino S, Kunimoto K, Muraoka Y, Mizushima Y, Katagiri K \& Tochino Y 1980 Breeding of a nonobese, diabetic strain of mice. Jikken Dobutsu 29 1-13.

Malaisse WJ, Malaisse-Lagae F, Sener A \& Pipeleers DG 1982 Determinants of the selective toxicity of alloxan to the pancreatic B cell. Proc.Natl.Acad.Sci.U.S.A. 79 927-930.

Marklund S \& Marklund G 1974 Involvement of the superoxide anion radical in the autoxidation of pyrogallol and a convenient assay for superoxide dismutase. Eur.J.Biochem. 47 469-474.

Marks DI \& Fox RM 1991 DNA damage, poly (ADP-ribosyl)ation and apoptotic cell death as a potential common pathway of cytotoxic drug action. Biochem.Pharmacol. 42 1859-1867.

Masutani M, Suzuki H, Kamada N, Watanabe M, Ueda O, Nozaki T, Jishage K, Watanabe T, Sugimoto T, Nakagama H, Ochiya T \& Sugimura T 1999 Poly (ADP-ribose) polymerase gene disruption conferred mice resistant to streptozotocin-induced diabetes. Proc.Natl.Acad.Sci.U.S.A 96 2301-2304.

Mathias S, Pena LA \& Kolesnick RN 1998 Signal transduction of stress via ceramide. Biochem.J. 335 ( Pt 3) $465-480$.

McAleer MA, Reifsnyder P, Palmer SM, Prochazka M, Love JM, Copeman JB, Powell EE, Rodrigues NR, Prins JB \& Serreze DV 1995 Crosses of NOD mice with the related NON strain. A polygenic model for IDDM. Diabetes 44 1186-1195.

McCord JM 1993 Human disease, free radicals, and the oxidant/antioxidant balance. Clin.Biochem. 26 351-357. 
McCord JM 2002 Superoxide dismutase in aging and disease: an overview. Methods Enzymol. 349 331341.

McKisic MD, Paturzo FX \& Smith AL 1996 Mouse parvovirus infection potentiates rejection of tumor allografts and modulates T cell effector functions. Transplantation 61 292-299.

Meyer T, Wierse G, Weinrebe W, Treuner J, Niethammer D \& Bruchelt G 1997 Effects of cyclophosphamide and ifosfamide on neuroblastoma cells before and after activation by microsomes. Anticancer Res. 17 981-986.

Michaud EJ, Bultman SJ, Klebig ML, van Vugt MJ, Stubbs LJ, Russell LB \& Woychik RP 1994 A molecular model for the genetic and phenotypic characteristics of the mouse lethal yellow (Ay) mutation. Proc.Natl.Acad.Sci.U.S.A 91 2562-2566.

Michaud EJ, Mynatt RL, Miltenberger RJ, Klebig ML, Wilkinson JE, Zemel MB, Wilkison WO \& Woychik RP 1997 Role of the agouti gene in obesity. J.Endocrinol. 155 207-209.

Minami T, Shimizu M, Tanaka H, Okazaki Y \& Cherian MG 1999 Metallothionein does not protect mouse endocrine cells from damage induced by alloxan injection. Toxicology 132 33-41.

Minegishi K, Tanaka M, Nishimura O, Tanigaki S, Miyakoshi K, Ishimoto H \& Yoshimura Y 2002 Reactive oxygen species mediate leukocyte-endothelium interactions in prostaglandin F2alpha -induced luteolysis in rats. Am.J.Physiol Endocrinol.Metab 283 E1308-E1315.

Moriscot C, Richard MJ, Favrot MC \& Benhamou PY 2003 Protection of insulin-secreting INS-1 cells against oxidative stress through adenoviral-mediated glutathione peroxidase overexpression. Diabetes Metab 29 145-151.

Murata M, Takahashi A, Saito I \& Kawanishi S 1999 Site-specific DNA methylation and apoptosis: induction by diabetogenic streptozotocin. Biochem.Pharmacol. 57 881-887.

Nagata M \& Yoon JW 1992 Studies on autoimmunity for T-cell-mediated beta-cell destruction. Distinct difference in beta-cell destruction between $\mathrm{CD} 4+$ and $\mathrm{CD} 8+\mathrm{T}$ - cell clones derived from lymphocytes infiltrating the islets of NOD mice. Diabetes 41 998-1008.

Nakamura H, Nakamura K \& Yodoi J 1997 Redox regulation of cellular activation. Annu.Rev.Immunol. 15 351-369.

Nakamura J, Hamada Y, Chaya S, Nakashima E, Naruse K, Kato K, Yasuda Y, Kamiya H, Sakakibara F, Koh N \& Hotta N 2002 Transition metals and polyol pathway in the development of diabetic neuropathy in rats. Diabetes Metab Res.Rev. 18 395-402.

Nathan CF 1987 Secretory products of macrophages. J.Clin.Invest. 79 319-326.

Nicklas W 1999 [Microbiological standardization of laboratory animals]. Berl Munch.Tierarztl.Wochenschr. 112 201-210.

Niklasson B, Heller KE, Schonecker B, Bildsoe M, Daniels T, Hampe CS, Widlund P, Simonson WT, Schaefer JB, Rutledge E, Bekris L, Lindberg AM, Johansson S, Ortqvist E, Persson B \& Lernmark A 2003 Development of type 1 diabetes in wild bank voles associated with islet autoantibodies and the novel ljungan virus. Int.J.Exp.Diabetes Res. 4 35-44. 
Nishikawa T, Edelstein D, Du XL, Yamagishi S, Matsumura T, Kaneda Y, Yorek MA, Beebe D, Oates PJ, Hammes HP, Giardino I \& Brownlee M 2000 Normalizing mitochondrial superoxide production blocks three pathways of hyperglycaemic damage. Nature 404 787-790.

Nishino T 1994 The conversion of xanthine dehydrogenase to xanthine oxidase and the role of the enzyme in reperfusion injury. J.Biochem. (Tokyo) 116 1-6.

Nohl H \& Jordan W 1986 The mitochondrial site of superoxide formation. Biochem.Biophys.Res.Commun. 138 533-539.

Nomikos IN, Wang Y \& Lafferty KJ 1989 Involvement of O2 radicals in 'autoimmune' diabetes. Immunol.Cell Biol. 67 85-87.

Nukatsuka M, Sakurai H, Yoshimura Y, Nishida M \& Kawada J 1988 Enhancement by streptozotocin of O2- radical generation by the xanthine oxidase system of pancreatic beta-cells. FEBS Lett. 239 295-298.

O'Neill SK, Dutta S \& Triggle CR 1993 Computerized data acquisition and analysis applied to chemiluminescence detection of nitric oxide in headspace gas. J.Pharmacol.Toxicol.Methods 29 217-221.

Oberley LW 1988 Free radicals and diabetes. Free Radic.Biol.Med. 5 113-124.

Ogawa Y, Kobayashi T, Nishioka A, Kariya S, Hamasato S, Seguchi H \& Yoshida S 2003 Radiationinduced reactive oxygen species formation prior to oxidative DNA damage in human peripheral $\mathrm{T}$ cells. Int.J.Mol.Med. 11 149-152.

Ohkuwa T, Sato Y \& Naoi M 1995 Hydroxyl radical formation in diabetic rats induced by streptozotocin. Life Sci. 56 1789-1798.

Ohly P, Dohle C, Abel J, Seissler J \& Gleichmann H 2000 Zinc sulphate induces metallothionein in pancreatic islets of mice and protects against diabetes induced by multiple low doses of streptozotocin. Diabetologia 43 1020-1030.

Ohly P \& Gleichmann H 1995 Metallothionein: in vitro induction with zinc and streptozotocin in pancreatic islets of mice. Exp.Clin.Endocrinol.Diabetes 103 Suppl 2 79-82.

Oldstone MB, Nerenberg M, Southern P, Price J \& Lewicki H 1991 Virus infection triggers insulindependent diabetes mellitus in a transgenic model: role of anti-self (virus) immune response. Cell $65319-$ 331.

Olefsky JM 2001 Prospects for research in diabetes mellitus. JAMA 285 628-632.

Orr WC \& Sohal RS 1994 Extension of life-span by overexpression of superoxide dismutase and catalase in Drosophila melanogaster. Science 263 1128-1130.

Oyadomari S, Araki E \& Mori M 2002 Endoplasmic reticulum stress-mediated apoptosis in pancreatic beta-cells. Apoptosis. 7 335-345.

Palmiter RD, Sandgren EP, Koeller DM \& Brinster RL 1993 Distal regulatory elements from the mouse metallothionein locus stimulate gene expression in transgenic mice. Mol.Cell Biol. 13 5266-5275.

Paolisso G, Tataranni PA, Foley JE, Bogardus C, Howard BV \& Ravussin E 1995 A high concentration of fasting plasma non-esterified fatty acids is a risk factor for the development of NIDDM. Diabetologia 38 1213-1217. 
Parsons PG, Lean J, Kable EP, Favier D, Khoo SK, Hurst T, Holmes RS \& Bellet AJ 1990 Relationships between resistance to cross-linking agents and glutathione metabolism, aldehyde dehydrogenase isozymes and adenovirus replication in human tumour cell lines. Biochem.Pharmacol. 40 2641-2649.

Peters GH, Frimurer TM \& Olsen OH 1998 Electrostatic evaluation of the signature motif $(H / V) C X 5 R$ $(\mathrm{S} / \mathrm{T})$ in protein-tyrosine phosphatases. Biochemistry 37 5383-5393.

Piercy V, Toseland CD \& Turner NC 2000 Acceleration of the development of diabetes in obese diabetic $(\mathrm{db} / \mathrm{db})$ mice by nicotinamide: a comparison with its antidiabetic effects in non-obese diabetic mice. Metabolism 49 1548-1554.

Pujol-Borrell R \& Bottazzo GF 1988 Puzzling diabetic transgenic mice: a lesson for human type 1 diabetes? Immunol.Today 9 303-306.

Rabinovitch A 1998 An update on cytokines in the pathogenesis of insulin-dependent diabetes mellitus. Diabetes Metab Rev. 14 129-151.

Rabinovitch A, Suarez WL \& Power RF 1993 Lazaroid antioxidant reduces incidence of diabetes and insulitis in nonobese diabetic mice. J.Lab.Clin.Med. 121 603-607.

Rabinovitch A, Suarez WL, Thomas PD, Strynadka K \& Simpson I 1992 Cytotoxic effects of cytokines on rat islets: evidence for involvement of free radicals and lipid peroxidation. Diabetologia 35 409-413.

Rabinovitch A \& Suarez-Pinzon WL 1998 Cytokines and their roles in pancreatic islet beta-cell destruction and insulin-dependent diabetes mellitus. Biochem.Pharmacol. 55 1139-1149.

Rabinovitch A, Suarez-Pinzon WL, Strynadka K, Lakey JR \& Rajotte RV 1996 Human pancreatic islet beta-cell destruction by cytokines involves oxygen free radicals and aldehyde production.

J.Clin.Endocrinol.Metab. 81 3197-3202.

Reddi AS \& Camerini-Davalos RA 1988 Hereditary diabetes in the KK mouse: an overview. Adv.Exp.Med.Biol. 246 7-15.

Robinson KA, Stewart CA, Pye Q, Floyd RA \& Hensley K 1999 Basal protein phosphorylation is decreased and phosphatase activity increased by an antioxidant and a free radical trap in primary rat glia. Arch.Biochem.Biophys. 365 211-215.

Rosen P, Nawroth PP, King G, Moller W, Tritschler HJ \& Packer L 2001 The role of oxidative stress in the onset and progression of diabetes and its complications: a summary of a Congress Series sponsored by UNESCO-MCBN, the American Diabetes Association and the German Diabetes Society. Diabetes Metab Res.Rev. 17 189-212.

Rossi F 1986 The O2- -forming NADPH oxidase of the phagocytes: nature, mechanisms of activation and function. Biochim. Biophys.Acta 853 65-89.

Saitoh M, Nishitoh H, Fujii M, Takeda K, Tobiume K, Sawada Y, Kawabata M, Miyazono K \& Ichijo H 1998 Mammalian thioredoxin is a direct inhibitor of apoptosis signal- regulating kinase (ASK) 1. EMBOJ. 17 2596-2606.

Sakuraba H, Mizukami H, Yagihashi N, Wada R, Hanyu C \& Yagihashi S 2002 Reduced beta-cell mass and expression of oxidative stress-related DNA damage in the islet of Japanese Type II diabetic patients. Diabetologia 45 85-96. 
Saldeen J, Lee JC \& Welsh N 2001 Role of p38 mitogen-activated protein kinase (p38 MAPK) in cytokine-induced rat islet cell apoptosis. Biochem.Pharmacol. 61 1561-1569.

Sanderson BJ \& Shield AJ 1996 Mutagenic damage to mammalian cells by therapeutic alkylating agents. Mutat.Res. 355 41-57.

Sandstrom J, Jonsson LM, Edlund H, Holmberg D \& Marklund SL 2002 Overexpression of extracellularSOD in islets of nonobese diabetic mice and development of diabetes. Free Radic.Biol.Med. 33 71-75.

Sarvetnick N, Liggitt D, Pitts SL, Hansen SE \& Stewart TA 1988 Insulin-dependent diabetes mellitus induced in transgenic mice by ectopic expression of class II MHC and interferon-gamma. Cell 52 773-782.

Sato M \& Bremner I 1993 Oxygen free radicals and metallothionein. Free Radic.Biol.Med 14 325-337.

Schnedl WJ, Ferber S, Johnson JH \& Newgard CB 1994 STZ transport and cytotoxicity. Specific enhancement in GLUT2- expressing cells. Diabetes 43 1326-1333.

Secrist JP, Burns LA, Karnitz L, Koretzky GA \& Abraham RT 1993 Stimulatory effects of the protein tyrosine phosphatase inhibitor, pervanadate, on T-cell activation events. J.Biol.Chem. 268 5886-5893.

Serreze DV, Chapman HD, Varnum DS, Hanson MS, Reifsnyder PC, Richard SD, Fleming SA, Leiter EH \& Shultz LD 1996 B lymphocytes are essential for the initiation of T cell-mediated autoimmune diabetes: analysis of a new "speed congenic" stock of NOD.Ig mu null mice. J.Exp.Med. 184 2049-2053.

Shah GM, Poirier D, Duchaine C, Brochu G, Desnoyers S, Lagueux J, Verreault A, Hoflack JC, Kirkland JB \& Poirier GG 1995 Methods for biochemical study of poly (ADP-ribose) metabolism in vitro and in vivo. Anal.Biochem. 227 1-13.

Shimabukuro M, Higa M, Zhou YT, Wang MY, Newgard CB \& Unger RH 1998a Lipoapoptosis in betacells of obese prediabetic fa/fa rats. Role of serine palmitoyltransferase overexpression. J.Biol.Chem. 273 32487-32490.

Shimabukuro M, Ohneda M, Lee Y \& Unger RH 1997 Role of nitric oxide in obesity-induced beta cell disease. J.Clin.Invest 100 290-295.

Shimabukuro M, Zhou YT, Levi M \& Unger RH 1998b Fatty acid-induced beta cell apoptosis: a link between obesity and diabetes. Proc.Natl.Acad.Sci.U.S.A 95 2498-2502.

Shimizu H, Uehara Y, Shimomura Y, Tanaka Y \& Kobayashi I 1991 Probucol attenuated hyperglycemia in multiple low-dose streptozotocin- induced diabetic mice. Life Sci. 49 1331-1338.

Siracusa LD 1994 The agouti gene: turned on to yellow. Trends Genet. 10 423-428.

Spagnoli A, Spadoni GL, Sesti G, Del Principe D, Germani D \& Boscherini B 1995 Effect of insulin on hydrogen peroxide production by human polymorphonuclear leukocytes. Studies with monoclonal antiinsulin receptor antibodies, and an agonist and an inhibitor of protein kinase C. Horm.Res. 43 286-293.

Strandell E, Buschard K, Saldeen J \& Welsh N 1995 Interleukin-1 beta induces the expression of hsp70, heme oxygenase and Mn-SOD in FACS-purified rat islet beta-cells, but not in alpha-cells. Immunol.Lett. 48 145-148.

Suarez-Pinzon WL, Mabley JG, Strynadka K, Power RF, Szabo C \& Rabinovitch A 2001 An inhibitor of inducible nitric oxide synthase and scavenger of peroxynitrite prevents diabetes development in nod mice. J.Autoimmun. 16 449-455. 
Suarez-Pinzon WL, Szabo C \& Rabinovitch A 1997 Development of autoimmune diabetes in NOD mice is associated with the formation of peroxynitrite in pancreatic islet beta-cells. Diabetes 46 907-911.

Sullivan SG, Chiu DT, Errasfa M, Wang JM, Qi JS \& Stern A 1994 Effects of H2O2 on protein tyrosine phosphatase activity in HER14 cells. Free Radic.Biol.Med. 16 399-403.

Sundaresan M, Yu ZX, Ferrans VJ, Irani K \& Finkel T 1995 Requirement for generation of H2O2 for platelet-derived growth factor signal transduction. Science 270 296-299.

Takada Y, Hachiya M, Park SH, Osawa Y, Ozawa T \& Akashi M 2002 Role of reactive oxygen species in cells overexpressing manganese superoxide dismutase: mechanism for induction of radioresistance.

Mol.Cancer Res. 1 137-146.

Takasu N, Komiya I, Asawa T, Nagasawa Y \& Yamada T 1991 Streptozocin- and alloxan-induced H2O2 generation and DNA fragmentation in pancreatic islets. $\mathrm{H} 2 \mathrm{O} 2$ as mediator for DNA fragmentation.

Diabetes 40 1141-1145.

Taketo M, Schroeder AC, Mobraaten LE, Gunning KB, Hanten G, Fox RR, Roderick TH, Stewart CL, Lilly F \& Hansen CT 1991 FVB/N: an inbred mouse strain preferable for transgenic analyses.

Proc.Natl.Acad.Sci.U.S.A. 88 2065-2069.

Tanaka, T, Gleason, C E, Harmon, J S, Tran, P O T, Oseid, E A, Hunter-Berger, K K, and Robertson, R. P. Prevention of glucose toxicity in HIT-T15 cells and ZDF rats in vivo by N-acetyl-l-cysteine and aminoguanidine. Diabetes 48 Supplement 1, 1037. 1999a.

Ref Type: Generic

Tanaka Y, Gleason CE, Tran PO, Harmon JS \& Robertson RP 1999b Prevention of glucose toxicity in HIT-T15 cells and Zucker diabetic fatty rats by antioxidants. Proc.Natl.Acad.Sci.U.S.A 96 10857-10862.

Tanaka Y, Tran PO, Harmon J \& Robertson RP 2002 A role for glutathione peroxidase in protecting pancreatic beta cells against oxidative stress in a model of glucose toxicity. Proc.Natl.Acad.Sci.U.S.A 99 12363-12368.

Tao J, Malbon CC \& Wang HY 2001 Insulin stimulates tyrosine phosphorylation and inactivation of protein-tyrosine phosphatase 1B in vivo. J.Biol.Chem. $27629520-29525$.

Tartaglia LA 1997 The leptin receptor. J.Biol.Chem. 272 6093-6096.

Tiedge M, Lortz S, Drinkgern J \& Lenzen S 1997 Relation between antioxidant enzyme gene expression and antioxidative defense status of insulin-producing cells. Diabetes 46 1733-1742.

Tiedge M, Lortz S, Munday R \& Lenzen S 1998 Complementary action of antioxidant enzymes in the protection of bioengineered insulin-producing RINm5F cells against the toxicity of reactive oxygen species. Diabetes 47 1578-1585.

Tiedge M, Lortz S, Munday R \& Lenzen S 1999 Protection against the co-operative toxicity of nitric oxide and oxygen free radicals by overexpression of antioxidant enzymes in bioengineered insulin-producing RINm5F cells. Diabetologia 42 849-855.

Tonks NK \& Neel BG 2001 Combinatorial control of the specificity of protein tyrosine phosphatases. Curr.Opin.Cell Biol. 13 182-195. 
Uchigata Y, Yamamoto H, Kawamura A \& Okamoto H 1982 Protection by superoxide dismutase, catalase, and poly (ADP-ribose) synthetase inhibitors against alloxan- and streptozotocin-induced islet DNA strand breaks and against the inhibition of proinsulin synthesis. J.Biol.Chem. 257 6084-6088.

Utsugi T, Yoon JW, Park BJ, Imamura M, Averill N, Kawazu S \& Santamaria P 1996 Major histocompatibility complex class I-restricted infiltration and destruction of pancreatic islets by NOD mouse-derived beta-cell cytotoxic CD8+ T-cell clones in vivo. Diabetes 45 1121-1131.

Vallee BL \& Falchuk KH 1993 The biochemical basis of zinc physiology. Physiol Rev. 73 79-118.

Venugopal SK, Devaraj S, Yang T \& Jialal I 2002 Alpha-tocopherol decreases superoxide anion release in human monocytes under hyperglycemic conditions via inhibition of protein kinase C-alpha. Diabetes $\mathbf{5 1}$ 3049-3054.

Verchere CB, D'Alessio DA, Palmiter RD, Weir GC, Bonner-Weir S, Baskin DG \& Kahn SE 1996 Islet amyloid formation associated with hyperglycemia in transgenic mice with pancreatic beta cell expression of human islet amyloid polypeptide. Proc.Natl.Acad.Sci.U.S.A. 93 3492-3496.

Vial CM, Ostlie DJ, Bhatti FN, Cozzi E, Goddard M, Chavez GP, Wallwork J, White DJ \& Dunning JJ 2000 Life supporting function for over one month of a transgenic porcine heart in a baboon. J.Heart Lung Transplant. $19224-229$.

von HM, Homann D, Gairin JE \& Oldstone MB 1997 Pathogenesis and treatment of virus-induced autoimmune diabetes: novel insights gained from the RIP-LCMV transgenic mouse model.

Biochem.Soc.Trans. 25 630-635.

Warbritton A, Gill AM, Yen TT, Bucci T \& Wolff GL 1994 Pancreatic islet cells in preobese yellow Avy/mice: relation to adult hyperinsulinemia and obesity. Proc.Soc.Exp.Biol.Med. 206 145-151.

Wei D, Fabris D \& Fenselau C 1999 Covalent sequestration of phosphoramide mustard by metallothionein-an in vitro study. Drug Metab Dispos. 27 786-791.

Welsh N, Eizirik DL \& Sandler S 1994a Nitric oxide and pancreatic beta-cell destruction in insulin dependent diabetes mellitus: don't take NO for an answer. Autoimmunity 18 285-290.

Welsh N, Makeeva N \& Welsh M 2002 Overexpression of the Shb SH2 domain-protein in insulinproducing cells leads to altered signaling through the IRS-1 and IRS-2 proteins. Mol.Med. 8 695-704.

Welsh N, Margulis B, Bendtzen K \& Sandler S 1994b Liposomal delivery of antioxidant enzymes protects against hydrogen peroxide- but not interleukin-1 beta-induced inhibition of glucose metabolism in rat pancreatic islets. J.Endocrinol. 143 151-156.

Welsh N, Margulis B, Bendtzen K \& Sandler S 1994c Liposomal delivery of antioxidant enzymes protects against hydrogen peroxide- but not interleukin-1 beta-induced inhibition of glucose metabolism in rat pancreatic islets. J.Endocrinol. 143 151-156.

Welsh N, Margulis B, Borg LA, Wiklund HJ, Saldeen J, Flodstrom M, Mello MA, Andersson A, Pipeleers DG \& Hellerstrom C 1995 Differences in the expression of heat-shock proteins and antioxidant enzymes between human and rodent pancreatic islets: implications for the pathogenesis of insulin-dependent diabetes mellitus. Mol.Med. 1 806-820.

Wheeler MB, Bleck GT \& Donovan SM 2001 Transgenic alteration of sow milk to improve piglet growth and health. Reprod.Suppl 58 313-324. 
White MF 2002 IRS proteins and the common path to diabetes. Am.J.Physiol Endocrinol.Metab 283 E413E422.

Wiseman H \& Halliwell B 1996 Damage to DNA by reactive oxygen and nitrogen species: role in inflammatory disease and progression to cancer. Biochem.J. 313 17-29.

Withers DJ, Burks DJ, Towery HH, Altamuro SL, Flint CL \& White MF 1999 Irs-2 coordinates Igf-1 receptor-mediated beta-cell development and peripheral insulin signalling. Nat.Genet. 23 32-40.

Wogensen L, Lee MS \& Sarvetnick N 1994 Production of interleukin 10 by islet cells accelerates immunemediated destruction of beta cells in nonobese diabetic mice. J.Exp.Med. 179 1379-1384.

Wu JJ, Chen X, Cao XC, Baker MS \& Kaufman DB 2001 Cytokine-induced metabolic dysfunction of MIN6 beta cells is nitric oxide independent. J.Surg.Res. 101 190-195.

Xu B, Moritz JT \& Epstein PN 1999 Overexpression of catalase provides partial protection to transgenic mouse beta cells. Free Radic.Biol.Med. 27 830-837.

Xue B, Moustaid N, Wilkison WO \& Zemel MB 1998 The agouti gene product inhibits lipolysis in human adipocytes via a Ca2+-dependent mechanism. FASEB J. 12 1391-1396.

Xue B \& Zemel MB 2001 Agouti signaling protein stimulates islet amyloid polypeptide (amylin) secretion in pancreatic beta-cells. Exp.Biol.Med. (Maywood.) 226 565-569.

Xue BZ, Wilkison WO, Mynatt RL, Moustaid N, Goldman M \& Zemel MB 1999 The agouti gene product stimulates pancreatic [beta]-cell Ca2+ signaling and insulin release. Physiol Genomics 1 11-19.

Yamamura K, Miyazaki T, Uno M \& Miyazaki J 1992 Transgenic mouse as a tool for the study of autoimmune disease: insulin-dependent diabetes mellitus. Int.J.Immunopharmacol. 14 451-455.

Yamashita N, Nishida M, Hoshida S, Kuzuya T, Hori M, Taniguchi N, Kamada T \& Tada M 1994 Induction of manganese superoxide dismutase in rat cardiac myocytes increases tolerance to hypoxia 24 hours after preconditioning. J.Clin.Invest 94 2193-2199.

Yan R, Peters LJ \& Travis EL 1991 Cyclophosphamide 24 hours before or after total body irradiation: effects on lung and bone marrow. Radiother. Oncol. 21 149-156.

Yasunami R \& Bach JF 1988 Anti-suppressor effect of cyclophosphamide on the development of spontaneous diabetes in NOD mice. Eur.J.Immunol. 18 481-484.

Zhang H, Ollinger K \& Brunk U 1995 Insulinoma cells in culture show pronounced sensitivity to alloxaninduced oxidative stress. Diabetologia 38 635-641.

Zhong W, Oberley LW, Oberley TD, Yan T, Domann FE \& St Clair DK 1996 Inhibition of cell growth and sensitization to oxidative damage by overexpression of manganese superoxide dismutase in rat glioma cells. Cell Growth Differ. 7 1175-1186.

Zhou CY, McInnes E, Parsons N, Langford G, Lancaster R, Richards A, Pino-Chavez G, Dos Santos CG, Copeman L, Carrington C \& Thompson S 2002 Production and characterization of a pig line transgenic for human membrane cofactor protein. Xenotransplantation. 9 183-190.

Zhou YP \& Grill VE 1994 Long-term exposure of rat pancreatic islets to fatty acids inhibits glucoseinduced insulin secretion and biosynthesis through a glucose fatty acid cycle. J.Clin.Invest 93 870-876. 
Zimmet P, Alberti KG \& Shaw J 2001 Global and societal implications of the diabetes epidemic. Nature $414782-787$.

Zimny S, Gogolin F, Abel J \& Gleichmann H 1993 Metallothionein in isolated pancreatic islets of mice: induction by zinc and streptozotocin, a naturally occurring diabetogen. Arch.Toxicol. 67 61-65. 


\section{APPEENDIX A}

\section{LIST OF ABBREVIATIONS}

NO

8-OHDG

AEC

$A G$

AGEs

AP-1

Ay gene

BB rat

BSA

Cat

CM- $\mathrm{H}_{2}$ DCFDA

$\mathrm{Cu} / \mathrm{Zn}$ SOD

CYP

DAB

$\mathrm{db}$

$\mathrm{db} / \mathrm{db}$ mice

ECSOD

EGF

ER

FBS

FFAs

$\mathrm{GHb}$

GK rat

GLUT2

$\mathrm{Gpx}$

$\mathrm{H}_{2} \mathrm{O}_{2}$

HBSS

HNE

$\mathrm{HO}$

$\mathrm{HX}$

Idd

IFN- $\gamma$

IL-1 $\beta$

iNOS

IRS

JNK

KRB buffer

Lepr $^{\mathrm{db}}$ gene nitric oxide

8-hydroxydeoxyguanosine

3-amino-ethylcarbazone

aminoguanidine

advanced glycosylation end-products

activating protein-1

Agouti gene

Bio-Breeding rat

bovine serum albumin

catalase

5-(6)-chloromethyl-2', 7'-dichlorodihydrofluorescein diacetate

Copper- and Zinc- containing superoxide dismutase

cyclophosphamide

3, 3'-diaminobenzidine

leptin receptor mutant allele

obese mice on C57BL/KsJ background containing homozygous

leptin receptor mutant gene

extracellular superoxide dismutase

epidermal growth factor

endoplasmic reticulum

fetal bovine serum

free fatty acids

glycohemoglobin

Goto-Kakizaki rat

glucose transporter 2

glutathione peroxidase

hydrogen peroxide

Hanks' balanced salt solution

4-hydroxy-nonenal

hydroxyl radical

hypoxanthine

diabetic susceptible allele

interferon-gamma

interleukin-l beta

inducible nitric oxide synthase

insulin receptor substrate

c-Jun- $\mathrm{N}$-terminal kinase

Krebs-Ringer bicarbonate buffer

leptin receptor mutant gene 


MDA
MnSOD
MPV
MT
MTT
NAC
NF-kB
NO
NOD mouse
NOS
O'
ob/ob mice
PARP
PBN
PBS
PDGF
PMNLs
PTPs
real time RT-PCR
RNS
ROS
SIN-1
SNAP
SOD
$\beta$-cell
STZ
TAE buffer
TNF- $\alpha$
Tpx
XO
ZDF rats

malondialdehyde

manganese superoxide dismutase

mouse parvovirus

metallothionein

3-[4,5-dimethylthiazol-2-yl]-diphenlyltetrazolium bromide $\mathrm{N}$-acetyl-L-cysteine

Nuclear factor-kappaB

nitric oxide

non-obese diabetic mouse

nitric oxide synthase

superoxide anion (radical)

obese mice on C57BL/6 background containing homozygous leptin ligand mutant gene

poly (ADP-ribose) polymerase

phenyl-N-tert-butylnitrone

phosphate-buffered saline

platelet-derived growth factor

human polymorphonuclear leukocytes

protein-tyrosine phosphatases

real time reverse transcriptase polymerase chain reaction

reactive nitrogen species

reactive oxygen species

3-morpholinosydnonimine

$\mathrm{S}$-nitro-N-acetyl-penicillamine

superoxide dismutases

pancreatic beta cell

streptozotocin

Tris-acetate-EDTA buffer

tumor necrosis factor-alpha

thioredoxin peroxidase

xanthine oxidase

zucker diabetic fat rats 


\section{CURRICULUM VITAE}

\section{Hainan Chen}

Address: $\quad 101$ Nob Hill Lane, Apt. 8, Louisville; KY 40206

Phone: $\quad 502-895-1782$ (Home); 502-852-2632 (Lab); Fax: 502-852-5634

Email: $\quad$ h0chen03@gwise.louisville.edu

\section{Education}

Shanghai Medical University (

Shanghai, P.R. China

$1987-1992$

Bachelor of Science in Pharmacology

University of North Dakota (UND)

Grand Forks, North Dakota

$1998-2000$

$\mathrm{Ph} . \mathrm{D}$. Student in Pharmacology program

University of Louisville (UofL)

Louisville, Kentucky

since 2001

$\mathrm{Ph}$.D. student in Pharmacology program

Master of Science in Pharmacology, August 2002

\section{Research Experience}

Huashan Hospital, SMU

Dept. of Biochemistry, SMU

Dept. of Pharmacology, UND

Dept. of Pharm.\& Toxi., UofL
Shanghai, P.R. China

1992

- Undergraduate thesis

"A study of the pharmacokinetics of a localmade ciprofloxacin in volunteers."

Shanghai, P.R. China

1992-1998

Research Assistant, working on:

- "The relationship of cancer development and glycosphingolipids" (1992-1996);

- "Depressive disorders concerning biochemical mechanisms and treatment in animal depressive models" (1997-1998);

Grand Forks, North Dakota

Louisville, Kentucky

1998-2000

Graduate Student, working on:

since 2001

- "Pancreatic beta cell antioxidant transgenes in diabetes and transplantation." 


\title{
Teaching
}

Dept. of Biochemistry, $\underline{\text { SMU }}$

Lecturer

$1992-1998$

- Taught undergraduate Biochemistry course and directed students' experiments.

\section{Publications \& Abstracts}

Hainan Chen, Yun Hu, Xia-Juan Xia. Variation of glycosidases during the development of DENAinduced rat hepatoma. Chinese Biochemical Journal (1993) 37:80-82

Xiaoyan Li, Chong-Bin Zhu, Hainan Chen, Yan-Hua Zhu, Gen-Cheng Wu, Shao-Fen Xu. Effects of fenfluramine combined with electroacupuncture on monoamine release in periaqueductual gray of rat brain. Acta Pharmacol Sin (1999) 20 (7): 597-600

Hainan Chen, Edward C. Carlson, Lori Pellet, Jon T. Moritz and Paul N. Epstein. Overexpression of Metallothionein in pancreatic beta cells reduces streptozotocin induced DNA damage and diabetes. Diabetes (2001) 50 (9): 2040-2046

Xiaoyan Li, Hainan Chen, Paul N. Epstein. Metallothionein protects islets from hypoxia and extends islet graft survival by scavenging most kinds of reactive oxygen species. The Journal of Biological Chemistry. (Submitted, co-first authors)

HainanChen, Xiaoyan Li, Paul N. Epstein. Overexpression of MnSOD and Catalase synergistically protects transgenic mouse beta cells from oxidative damage but not from cytokine-induced cytotoxicity. Manuscript in preparation.

\begin{abstract}
Absracts
Xiaoyan Li, Hainan Chen, Paul N. Epstein. Metallothionein protects islets from hypoxia and extends islet graft survival by scavenging most kinds of reactive oxygen species. American Diabetes Association's $63^{\text {rd }}$ Scientific Sessions; Jun. 2003 (New Orleans, Louisiana)

Xiaoyan Li, Hainan Chen, Patricia Kralik, Paul N. Epstein. Unexpected susceptibility to cyclophosphamide accelerated Type 1 diabetes and beta cell apoptosis in NOD mice due to beta cell overexpression of antioxidant transgenes" -.-- LATE BREAKING ABSTRACT. American Diabetes Association's 6 ${ }^{\text {rd }}$ Scientific Sessions; Jun. 2003 (New Orleans, Louisiana)
\end{abstract}

HainanChen, Xiaoyan Li, Paul N. Epstein Paul N. Epstein. Overexpression of MnSOD and Catalase Synergistically protects transgenic mouse beta cells from oxidative damage but not from cytokine-induced cytotoxicity. American Diabetes Association's 63 ${ }^{\text {rd }}$ Scientific Sessions; Jun. 2003 (New Orleans, Louisiana)

\section{Honors}

CGeMM fellowship, 2001-2003, University of Louisville 